\title{
Relative Orbit Determination For Autonomous Close Formation Flying Spacecraft
}

\author{
by
}

\section{Ramin Tavvafi}

\author{
Coursework, Carleton University \\ B.Sc., Isfahan University of Technology
}

A thesis submitted to

the Faculty of Graduate Studies and Research

in partial fulfillment of

the requirements for the degree of

Master of Applied Science

Ottawa-Carleton Institute for Mechanical and Aerospace Engineering

Department of Mechanical and Aerospace Engineering

Carleton University

Ottawa, Ontario

Canada

January 2010 
Library and Archives Canada

Published Heritage Branch

395 Wellington Street Ottawa ON K1A ON4 Canada
Bibliotheque et

Archives Canada

Direction du

Patrimoine de l'édition

395, rue Wellington

Ottawa ON K1A ON4

Canada
NOTICE:

The author has granted a nonexclusive license allowing Library and Archives Canada to reproduce, publish, archive, preserve, conserve, communicate to the public by telecommunication or on the Internet, loan, distribute and sell theses worldwide, for commercial or noncommercial purposes, in microform, paper, electronic and/or any other formats.

The author retains copyright ownership and moral rights in this thesis. Neither the thesis nor substantial extracts from it may be printed or otherwise reproduced without the author's permission.
Your file Votre reférence ISBN: 978-0-494-68643-0 Our file Notre reférence ISBN: 978-0-494-68643-0
In compliance with the Canadian Privacy Act some supporting forms may have been removed from this thesis.

While these forms may be included in the document page count, their removal does not represent any loss of content from the thesis.
AVIS:

L'auteur a accordé une licence non exclusive permettant à la Bibliothèque et Archives Canada de reproduire, publier, archiver, sauvegarder, conserver, transmettre au public par télécommunication ou par l'Internet, prêter, distribuer et vendre des thèses partout dans le monde, à des fins commerciales ou autres, sur support microforme, papier, électronique et/ou autres formats.

L'auteur conserve la propriété du droit d'auteur et des droits moraux qui protège cette thèse. $\mathrm{Ni}$ la thèse ni des extraits substantiels de celle-ci ne doivent être imprimés ou autrement reproduits sans son autorisation.

\section{Canadä}




\section{Acknowledgements}

I am deeply indebted to a number of people for all their helps and supports in performing this thesis. First of all I would like to thank my research advisor, professor Jurek Sasiadek whose constant guidance and directions made the thesis a true learning experience for me.

I would also like to express my gratitude to professor Kyle T. Alfriend from Texas A\&M university, for his invaluable assistances on the formulation of spacecrafts relative motion theory. I also wish to thank professor Penina Axelrad from university of Colorado for helping me to understand the characteristics of LVLH curvilinear frame. I furthermore appreciate professor Jean de Lafontaine and his Ph.D. student Jean-Francois Hamel from university of Sherbrooke for their sage suggestions on my thesis relative motion simulations.

Finally I am extremely grateful to my wife Mojgan for her love and support during all those long hours which I spent with the computer instead of her. 


\begin{abstract}
Formation flying of multiple spacecrafts is an enabling technology for many future space missions. It allows a few small cost-effective satellites to offer capabilities that are only achievable with a much more expensive single satellite. While spacecraft formation flying provides many operational and performance advantages, it also poses many significant challenges in navigation, guidance, and control. One of the key challenges for a formation flying satellite is to have a very precise and efficient autonomous relative navigation algorithm implemented on a typical computationally restricted on-board computer. This autonomous relative navigation system is a crucial requirement which enables other key components like autonomous guidance and control systems to plan new trajectories and command the satellite movements during any formation keeping or formation reconfiguration maneuvers.

This thesis investigates the autonomous relative navigation problem for formation flying spacecrafts. A highly efficient autonomous relative orbit determination method is developed and numerically evaluated for centralized-controlled close formation flying satellites. In centralized-controlled formation flight, one chief satellite is responsible for simultaneous navigation, guidance and control of all the remaining deputy satellites. The proposed relative orbit determination method employs real-time observation data generated by an active phased array radar and iterated extended Kalman filters to estimate the relative orbit states of all the deputy satellites.
\end{abstract}




\section{Contents}

$\begin{array}{ll}\text { Acknowledgements } & \text { ii }\end{array}$

Abstract $\quad$ iii

List of Figures vi

List of Tables $\quad$ ix

List of Symbols $\quad x$

Chapter 1. Introduction 1

1.1 Thesis Objective . . . . . . . . . . . . . . . . . . . 7

1.2 Thesis Overview . . . . . . . . . . . . . . . 7

Chapter 2. Relative Orbit Description For Formation Flying Spacecraft

2.1 General Relative Orbit Description . . . . . . . . . . . . . . 8

2.1.1 LVLH Rectilinear Frame Coordinate Description . . . . . . . . 10

2.1.2 Orbit Element Difference Description . . . . . . . . . . . . . 16

2.1.3 Linear Mapping between LVLH Rectilinear Frame Coordinates and Orbit Element Differences . . . . . . . . . . . . 18

2.2 Relative Motion State Transition Matrix for the perturbed Chief Orbit 24

2.2.1 Gim-Alfriend Geometric Method ... . . . . . . . . 25

2.2 .2 Instantaneous Transformation matrix $\mathcal{T} \ldots \ldots . \ldots . . . .29$

2.2.3 State Transition Matrix for the Relative Mean Orbit Elements

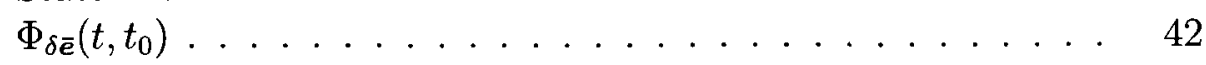

2.2.4 Transformation Matrix Between the Relative Mean and the Relative Osculating Orbit Elements $D(t) \ldots \ldots \ldots . \ldots 46$

2.3 Numerical Evaluation . . . . . . . . . . . . . . . . . . . . . . 49

2.3 .1 Simulation Test Plans . . . . . . . . . . . . . . . . 59

2.3.2 Satellites Close Formation Flight Test Case 1 . . . . . . . . 61

2.3.3 Satellites Close Formation Flight Test Case $2 \ldots . . . . .70$

2.3.4 Satellites Far Formation Flight Test Case 3 . . . . . . . . . 79

Chapter 3. Radar Observation and Relative Orbit State Estimation 90

3.1 Extended Kalman Filter . . . . . . . . . . . . . . . . . . . . . 94

3.2 Iterated Extended Kalman Filter . . . . . . . . . . . . . . . . . . . 99 
3.3 Numerical Evaluation . . . . . . . . . . . . . . . . . . . . 101

3.3.1 Simulation Test Plans . . . . . . . . . . . . . . . . . . 102

3.3.2 Deputy Satellites Relative Orbit Determinations for Test Case 1106

3.3.3 Deputy Satellites Relative Orbit Determinations for Test Case 2116

3.3.4 Deputy Satellites Relative Orbit Determinations for Test Case 3126

Chapter 4. Summary of Simulation Results, Conclusions and Future Researches

4.1 Summary of Simulation Results . . . . . . . . . . . . . . . . . 136

4.2 Conclusions . . . . . . . . . . . . . . . . . . . . 139

4.3 Recommendations for Future Researches . . . . . . . . . . . . . 140

$\begin{array}{ll}\text { References } & 142\end{array}$

$\begin{array}{lll}\text { Appendix A. Disturbed Two-Body Motions Identities } & 146\end{array}$

$\begin{array}{lll}\text { Appendix B. Transformation Matrix } \mathcal{T} & 150\end{array}$

Appendix C. Analytical Inverse of Transformation Matrix $\mathcal{T} \quad 152$

Appendix D. Mean State Transition Matrix $\Phi_{\delta \bar{e}}\left(t, t_{0}\right) \quad 155$

Appendix E. Orbit Elements Transformation Column Vectors $e^{(l p)}$, $e^{(s p 1)}$, and $e^{(s p 2)}$

159

E.1 Long Period Oscillations Column Vector $e^{(l p)} \ldots \ldots \ldots$. . . . . . 160

E.2 Short Period 1 Oscillations Column Vector $\boldsymbol{e}^{(s p 1)} \ldots \ldots \ldots \ldots$. . . . . 161

E.3 Short Period 2 Oscillations Column Vector $e^{(s p 2)} \ldots \ldots . . . . .162$

Appendix F. Relative Orbit Elements Transformation Matrices $D^{(l p)}$, $D^{(s p 1)}$, and $D^{(s p 2)}$

164

F.1 Long Period Oscillations Matrix $D^{(l p)} \ldots \ldots \ldots \ldots \ldots$. . . . . 164

F.2 Short Period 1 Oscillations Matrix $D^{(s p 1)} \ldots \ldots \ldots$. . . . . . 167

F.3 Short Period 2 Oscillations Matrix $D^{(s p 2)} \ldots \ldots \ldots \ldots$ 


\section{List of Figures}

1.1 Relative motion theories classification . . . . . . . . . . . 6

2.1 Illustration of a general type of two-spacecraft formation . . . . . . . 9

2.2 Illustration of a general type of spacecraft formation in the chief LVLH

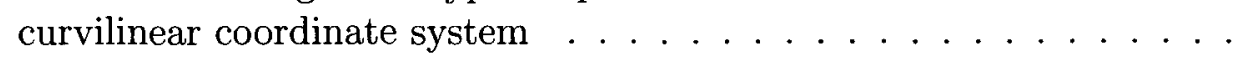

2.3 Illustration of three consecutive rotations through Euler's angles $\Omega, i$, and $\theta$ of a body-axes triad . . . . . . . . . . . . .

2.4 Illustration of the relationship between the eccentric argument of latitude $F$ and the true argument of latitude $\theta \ldots \ldots \ldots$

2.5 Illustration of the deputy satellite relative coordinates in the chief LVLH curvilinear frame . . . . . . . . . . . . . . .

2.6 Illustration of the chief and deputy satellites' inertial and relative orbit trajectories in the test case $1 \ldots \ldots \ldots$

2.7 Illustration of the deputy satellite 1 actual/predicted relative position and velocity vectors components in the test case $1 \ldots \ldots$. . . .

2.8 Illustration of the deputy satellite 1 relative position and velocity errors in the test case $1 \ldots \ldots \ldots \ldots \ldots$

2.9 Illustration of the deputy satellite 2 actual/predicted relative position and velocity vectors components in the test case $1 \ldots \ldots$. . . .

2.10 Illustration of the deputy satellite 2 relative position and velocity errors in the test case $1 \ldots \ldots \ldots \ldots$

2.11 Illustration of the chief and deputy satellites' inertial and relative orbit trajectories in the test case $2 \ldots \ldots \ldots \ldots$. . . . . . . .

2.12 Illustration of the deputy satellite 1 actual/predicted relative position and velocity vectors components in the test case $2 \ldots \ldots$. . . .

2.13 Illustration of the deputy satellite 1 relative position and velocity errors in the test case $2 \ldots \ldots \ldots \ldots \ldots$

2.14 Illustration of the deputy satellite 2 actual/predicted relative position and velocity vectors components in the test case $2 \ldots \ldots$. . . .

2.15 Illustration of the deputy satellite 2 relative position and velocity errors in the test case $2 \ldots \ldots \ldots \ldots \ldots$

2.16 Illustration of the chief and deputy satellites' inertial and relative orbit trajectories in the test case $3 \ldots \ldots \ldots$

2.17 Illustration of the deputy satellite 1 actual/predicted relative position and velocity vectors components in the test case $3 \ldots \ldots \ldots$

9

26 
2.18 Illustration of the deputy satellite 1 relative position and velocity errors in the test case $3 \ldots \ldots \ldots \ldots \ldots$

2.19 Illustration of the deputy satellite 2 actual/predicted relative position and velocity vectors components in the test case $3 \ldots \ldots$. . . . .

2.20 Illustration of the deputy satellite 2 relative position and velocity errors in the test case $3 \ldots \ldots \ldots \ldots$

3.1 Geometry of the deputy satellite radar observation in the chief rectilinear LVLH frame . . . . . . . . . . . . . . . . . . . .

3.2 Illustration of an example of Gaussian noise added to slant range vector observations . . . . . . . . . . . . . . .

3.3 Illustration of the deputy satellite 1 rectilinear versus curvilinear relative position vector differences in the test case $1 \ldots \ldots . . .$.

3.4 Illustration of the deputy satellite 2 rectilinear versus curvilinear relative position vector differences in the test case $1 \ldots \ldots . . .$.

3.5 Illustration of the deputy satellite 1 slant range vector observations data in the test case $1 \ldots \ldots \ldots \ldots$

3.6 Illustration of the deputy satellite 1 actual/estimated relative position and velocity vectors components in the test case $1 \ldots \ldots$. . . . .

3.7 Illustration of the deputy satellite 1 relative position and velocity estimation errors in the test case $1 \ldots \ldots . . . \ldots$

3.8 Illustration of the deputy satellite 2 slant range vector observations data in the test case $1 \ldots \ldots \ldots \ldots$

3.9 Illustration of the deputy satellite 2 actual/estimated relative position and velocity vectors components in the test case $1 \ldots \ldots . .$.

3.10 Illustration of the deputy satellite 2 relative position and velocity estimation errors in the test case $1 \ldots \ldots \ldots . . . \ldots$

3.11 Illustration of the deputy satellite 1 rectilinear versus curvilinear relative position vector differences in the test case $2 \ldots \ldots$. . . . . 117

3.12 Illustration of the deputy satellite 2 rectilinear versus curvilinear relative position vector differences in the test case $2 \ldots \ldots . . .$.

3.13 Illustration of the deputy satellite 1 slant range vector observations data in the test case $2 \ldots \ldots \ldots \ldots$

3.14 Illustration of the deputy satellite 1 actual/estimated relative position and velocity vectors components in the test case $2 \ldots \ldots . .$.

3.15 Illustration of the deputy satellite 1 relative position and velocity estimation errors in the test case $2 \ldots \ldots \ldots$

3.16 Illustration of the deputy satellite 2 slant range vector observations data in the test case $2 \ldots \ldots \ldots \ldots \ldots$

3.17 Illustration of the deputy satellite 2 actual/estimated relative position and velocity vectors components in the test case $2 \ldots \ldots$. . . . .

3.18 Illustration of the deputy satellite 2 relative position and velocity estimation errors in the test case $2 \ldots \ldots \ldots$

3.19 Illustration of the deputy satellite 1 rectilinear versus curvilinear relative position vector differences in the test case $3 \ldots \ldots \ldots$ 
3.20 Illustration of the deputy satellite 2 rectilinear versus curvilinear relative position vector differences in the test case $3 \ldots \ldots$. . . . . . 127

3.21 Illustration of the deputy satellite 1 slant range vector observations data in the test case $3 \ldots \ldots$. . . . . . . . . . . . 129

3.22 Illustration of the deputy satellite 1 actual/estimated relative position and velocity vectors components in the test case $3 \ldots \ldots 130$

3.23 Illustration of the deputy satellite 1 relative position and velocity estimation errors in the test case $3 \ldots \ldots \ldots \ldots$

3.24 Illustration of the deputy satellite 2 slant range vector observations data in the test case $3 \ldots \ldots \ldots \ldots$

3.25 Illustration of the deputy satellite 2 actual/estimated relative position and velocity vectors components in the test case $3 \ldots \ldots$. . . . . 134

3.26 Illustration of the deputy satellite 2 relative position and velocity estimation errors in the test case $3 \ldots \ldots \ldots . \ldots 135$ 


\section{List of Tables}

2.1 Test case 1 initial conditions for the chief and deputy satellite $1 \ldots 61$

2.2 Test case 1 initial conditions for the chief and deputy satellite $2 \ldots 61$

2.3 Test case 2 initial conditions for the chief and deputy satellite $1 \ldots 70$

2.4 Test case 2 initial conditions for the chief and deputy satellite $2 \ldots 70$

2.5 Test case 3 initial conditions for the chief and deputy satellite 1 . . 79

2.6 Test case 3 initial conditions for the chief and deputy satellite $2 \ldots 79$ 


\section{List of Symbols}

\begin{tabular}{cl} 
Symbol & Description \\
\hline $\boldsymbol{r}_{c}$ & Chief satellite inertial position vector \\
$\boldsymbol{r}_{d}$ & Deputy satellite inertial position vector \\
$\mathcal{O}$ & Chief satellite LVLH rectilinear frame \\
$\mathcal{O}_{\mathcal{D}}$ & Deputy satellite LVLH rectilinear frame \\
$\hat{\boldsymbol{o}}_{r}$ & Radial unit vector of the chief satellite LVLH rectilinear frame \\
$\hat{\boldsymbol{o}}_{\theta}$ & In-track unit vector of the chief satellite LVLH rectilinear frame \\
$\hat{\boldsymbol{o}}_{h}$ & Cross-track unit vector of the chief satellite LVLH rectilinear frame \\
$\hat{\boldsymbol{o}}_{r, d}$ & Radial unit vector of the deputy satellite LVLH rectilinear frame \\
$\hat{\boldsymbol{o}}_{\theta, d}$ & In-track unit vector of the deputy satellite LVLH rectilinear frame \\
$\hat{\boldsymbol{o}}_{h, d}$ & Cross-track unit vector of the deputy satellite LVLH rectilinear frame \\
$\boldsymbol{h}$ & Chief orbit angular momentum vector \\
$\boldsymbol{\rho}$ & Deputy satellite relative position vector in the chief satellite LVLH \\
$\mathcal{N}$ & $\quad$ rectilinear frame \\
$p$ & Earth centered inertial frame \\
$\mu$ & Chief satellite orbit semiparameter \\
$a$ & Earth gravitational coefficient \\
$e$ & Chief satellite orbit semimajor axis \\
$i$ & Chief satellite orbit eccentricity \\
$\Omega$ & Chief satellite orbit inclination \\
$\omega$ & Chief satellite orbit longitude of the ascending node \\
$M$ & Chief satellite orbit argument of perigee \\
$n$ & Chief satellite orbit mean anomaly \\
$f$ & Chief satellite orbit mean motion \\
$E$ & Chief satellite orbit true anomaly \\
$F$ & Chief satellite orbit eccentric anomaly \\
& Chief satellite orbit argument of latitude \\
& Chief satellite orbit mean argument of latitude \\
& Chief satellite orbit eccentric argument of latitude \\
\hline &
\end{tabular}




\begin{tabular}{|c|c|}
\hline Symbol & Description \\
\hline $\boldsymbol{X}_{\rho}$ & $\begin{array}{l}\text { Deputy satellite relative state vector in the chief satellite LVLH } \\
\text { rectilinear frame }\end{array}$ \\
\hline $\boldsymbol{e}_{c}$ & Chief satellite orbit element vector \\
\hline $\boldsymbol{e}_{d}$ & Deputy satellite orbit element vector \\
\hline$\delta e$ & Deputy satellite relative orbit element vector \\
\hline$[\mathcal{O N}]$ & $\begin{array}{l}\text { Direction cosine matrix mapping any vector components in the } \\
\text { inertial frame to its components in the chief satellite LVLH } \\
\text { rectilinear frame }\end{array}$ \\
\hline$\left[\mathcal{O}_{\mathcal{D}} \mathcal{N}\right]$ & $\begin{array}{l}\text { Direction cosine matrix mapping any vector components in the } \\
\text { inertial frame to its components in the deputy satellite LVLH } \\
\text { rectilinear frame }\end{array}$ \\
\hline $\boldsymbol{r}_{d}^{\mathcal{O}}$ & $\begin{array}{l}\text { Deputy satellite inertial position vector in terms of the chief } \\
\text { satellite LVLH rectilinear frame components }\end{array}$ \\
\hline$r_{d}^{\mathcal{O}_{\mathcal{D}}}$ & $\begin{array}{l}\text { Deputy satellite inertial position vector in terms of the deputy } \\
\text { satellite LVLH rectilinear frame components }\end{array}$ \\
\hline$[\mathcal{R}]$ & Rotation matrix \\
\hline$V_{r}$ & Chief satellite velocity vector radial component in Keplerian motion \\
\hline$V_{t}$ & $\begin{array}{l}\text { Chief satellite velocity vector tangential component in Keplerian } \\
\text { motion }\end{array}$ \\
\hline$O$ & Chief satellite LVLH curvilinear frame \\
\hline$O_{\mathcal{D}}$ & Deputy satellite LVLH curvilinear frame \\
\hline$\hat{\boldsymbol{e}}_{x}$ & Radial unit vector of the chief satellite LVLH curvilinear frame \\
\hline$\hat{\boldsymbol{e}}_{y}$ & In-track unit vector of the chief satellite LVLH curvilinear frame \\
\hline$\hat{\boldsymbol{e}}_{z}$ & Cross-track unit vector of the chief satellite LVLH curvilinear frame \\
\hline$J_{2}$ & Earth first zonal gravitational harmonic coefficient \\
\hline $\boldsymbol{X}$ & $\begin{array}{l}\text { Deputy satellite relative state vector in the chief satellite LVLH } \\
\text { curvilinear frame }\end{array}$ \\
\hline$\varpi$ & $\begin{array}{l}\text { Instantaneous total angular velocity vector of the chief satellite } \\
\text { LVLH curvilinear frame }\end{array}$ \\
\hline$R_{e}$ & Earth equatorial radius \\
\hline$\overline{\boldsymbol{X}}$ & $\begin{array}{l}\text { Deputy satellite relative mean state vector in the chief satellite } \\
\text { LVLH curvilinear frame }\end{array}$ \\
\hline$\overline{\boldsymbol{e}}_{c}$ & Chief satellite mean orbit element vector \\
\hline$\overline{\boldsymbol{e}}_{d}$ & Deputy satellite mean orbit element vector \\
\hline$\delta \overline{\boldsymbol{e}}$ & Deputy satellite relative mean orbit element vector \\
\hline$\Phi_{\delta \boldsymbol{e}}$ & State transition matrix for the relative osculating orbit elements \\
\hline$\Phi_{\delta \bar{e}}$ & State transition matrix for the relative mean orbit elements \\
\hline
\end{tabular}




\begin{tabular}{|c|c|}
\hline Symbol & Description \\
\hline$\Phi_{X}$ & $\begin{array}{l}\text { Gim-Alfriend state transition matrix for the relative instantaneous } \\
\text { motion }\end{array}$ \\
\hline$D$ & $\begin{array}{l}\text { Transformation matrix to map the relative mean orbit elements to } \\
\text { the relative osculating orbit elements }\end{array}$ \\
\hline $\bar{\varpi}$ & $\begin{array}{l}\text { Mean total angular velocity vector of the chief satellite LVLH } \\
\text { curvilinear frame }\end{array}$ \\
\hline $\mathcal{T}$ & $\begin{array}{l}\text { Transformation matrix to map the relative osculating orbit elements } \\
\text { to relative state vector in the chief satellite LVLH curvilinear frame }\end{array}$ \\
\hline$V_{r J_{2}}$ & $\begin{array}{l}\text { Chief satellite velocity vector radial component in } J_{2} \text { perturbed } \\
\text { motion }\end{array}$ \\
\hline$V_{t J_{2}}$ & $\begin{array}{l}\text { Chief satellite velocity vector tangential component in } J_{2} \text { perturbed } \\
\text { motion }\end{array}$ \\
\hline$V_{n J_{2}}$ & $\begin{array}{l}\text { Chief satellite velocity vector normal component in } J_{2} \text { perturbed } \\
\text { motion }\end{array}$ \\
\hline$[O \mathcal{N}]$ & $\begin{array}{l}\text { Direction cosine matrix mapping any vector components in the } \\
\text { inertial frame to its components in the chief satellite LVLH } \\
\text { curvilinear frame }\end{array}$ \\
\hline$\left[O_{\mathcal{D}} \mathcal{N}\right]$ & $\begin{array}{l}\text { Direction cosine matrix mapping any vector components in the } \\
\text { inertial frame to its components in the deputy satellite LVLH } \\
\text { curvilinear frame }\end{array}$ \\
\hline $\boldsymbol{r}_{d}^{O}$ & $\begin{array}{l}\text { Deputy satellite inertial position vector in terms of the chief } \\
\text { satellite LVLH curvilinear frame components }\end{array}$ \\
\hline$r_{d}^{O_{\mathcal{D}}}$ & $\begin{array}{l}\text { Deputy satellite inertial position vector in terms of the deputy } \\
\text { satellite LVLH curvilinear frame components }\end{array}$ \\
\hline $\boldsymbol{v}_{d}^{O}$ & $\begin{array}{l}\text { Deputy satellite inertial velocity vector in terms of the chief } \\
\text { satellite LVLH curvilinear frame components }\end{array}$ \\
\hline $\boldsymbol{v}_{d}^{O_{\mathcal{D}}}$ & $\begin{array}{l}\text { Deputy satellite inertial velocity vector in terms of the deputy } \\
\text { satellite LVLH curvilinear frame components }\end{array}$ \\
\hline $\mathscr{R}$ & Disturbance potential function in Lagrange's planetary equations \\
\hline $\bar{a}$ & Chief satellite orbit mean semimajor axis \\
\hline $\bar{e}$ & Chief satellite orbit mean eccentricity \\
\hline $\bar{i}$ & Chief satellite orbit mean inclination \\
\hline $\bar{\Omega}$ & Chief satellite orbit mean longitude of the ascending node \\
\hline $\bar{\omega}$ & Chief satellite orbit mean argument of perigee \\
\hline $\bar{M}$ & Chief satellite orbit mean mean anomaly \\
\hline$U$ & Earth gravity potential function \\
\hline
\end{tabular}




\begin{tabular}{cl} 
Symbol & Description \\
\hline $\boldsymbol{Z}$ & Radar observation data of the deputy satellite obtained in the chief \\
& satellite LVLH rectilinear frame \\
$\rho$ & Radar range observation data of the deputy satellite \\
$e l$ & Radar azimuth observation data of the deputy satellite \\
$\boldsymbol{h}$ & Radar elevation observation data of the deputy satellite \\
& Mathematical model which relates the radar observations of the \\
& deputy satellite to its relative states in the chief satellite LVLH \\
$H$ & curvilinear frame \\
& Measurement matrix which relates changes in the observation data of \\
$\boldsymbol{W}$ & the deputy satellite to changes in its relative states \\
$\boldsymbol{V}$ & Zero-mean white Gaussian noise process for system model \\
$Q$ & Zero-mean white Gaussian noise process for measurement model \\
$R$ & Measurement noise covariance matrix \\
$E$ & Expected value of a random process \\
$\hat{\boldsymbol{X}}$ & Deputy satellite relative state estimate \\
$\hat{\boldsymbol{X}}^{-}$ & Deputy satellite a priori relative state estimate \\
$\hat{\boldsymbol{X}}^{+}$ & Deputy satellite a posteriori relative state estimate \\
$\tilde{\boldsymbol{X}}^{-}$ & Deputy satellite relative state estimation error \\
$P^{-}$ & Deputy satellite a priori estimation error covariance matrix \\
$P^{+}$ & Deputy satellite a posteriori estimation error covariance matrix \\
$K$ & Extended Kalman filter gain matrix
\end{tabular}




\section{Chapter 1}

\section{Introduction}

Formation flying of satellites has become an emerging technology for the next generation of space systems in twenty-first century. It describes how multiple satellites fly together in close proximity and how they work as a group to accomplish a single mission objective. There are many significant advantages in employing formation flying satellites to carry out any space mission. Some of these benefits are as follows

- A constellation of a few small cost-effective satellites can offer capabilities that are only achievable with a much more expensive single satellite.

- There will be no need to do intensive planning and calculations in groundbased control room to perform a satellite orbit maneuvers. Satellites flying in formation will have the capability to react to each other orbit changes and maintain their relative orbit closeness without human intervention.

- Formation flying satellites provide for graceful degradation of performance during times of a satellite failure. If a single large satellite has a permanent system malfunction, the entire mission is at risk. If a satellite in a formation fails, the remaining satellites in the formation may continue to perform the mission at a lower performance level. The complete formation could then later be brought back up to mission design specifications with the addition of another inexpensive replacement satellite. 
- A space mission objective for formation flying satellites can be substantially altered by only reconfiguring the satellites arrangement in the formation.

- Formation flying satellites can provide scientists with the necessary means to obtain very precise measurements data by just combining sensors informations from several small satellites.

- Formation flying satellites are able to produce many new types of scientific data which are often unavailable from a single satellite alone. For instance they can simultaneously supply imagery data of the same ground scene from different observation angles in space.

Some of the applications commonly proposed for the formation flying satellites are Earth surveillance, remote sensing, space-based radar, ground-based terrestrial laser communication system and stellar imaging. For most applications the maximum separation distance between two satellites must be less than a few kilometers. Formation flying satellites often consist of a central chief satellite surrounded by multiple deputy satellites all concurrently orbiting a planet. In some formations a central chief satellite may not physically exist. For those cases, an imaginary chief satellite provides a virtual reference point for the rest of the formation. In this thesis however, we will only concentrate on autonomous centralized-controlled satellites formation flights which they do require a central chief satellite to be physically present and this chief satellite is responsible for navigation, guidance and control of all the deputy satellites in the formation.

There has been enormous research efforts mostly during past ten years to understand and solve problems related to the development and implementation of guidance and control algorithms for the autonomous formation flying satellites. Autonomous guidance and control is an essential requirement for all of the satellites formation flying applications. It is clear that maneuvers required for satellites formation keeping 
or satellites formation reconfiguration are very complex and due to communication delay between Earth and the satellites, satellites maneuvers can not be commanded at the low-level from the ground stations. When a satellite flies in a rapidly evolving space environment and specially in close vicinity of other satellites, the reaction time of guidance and control system becomes very critical. Therefore this reaction time must be minimized by removing the ground support from the control loop. Guidance algorithms by definition are responsible for planning trajectories and devising open-loop control law for each deputy satellite. Control algorithms on the other hand couples the dynamic states of all the deputy satellites through one common optimal closed-loop control law and direct each deputy satellite maneuver over its planned trajectory. References [1] and [2] provide a comprehensive survey of autonomous guidance and control methods devised for formation flying satellites up to year 2004 .

On the contrast with numerous autonomous guidance and control methods developed for the formation flying satellites, there have been only a few research efforts to identify and explore new algorithms for the autonomous navigation of formation flying spacecrafts. The prime reason for the lack of research interest in autonomous space navigation is the availability of the global positioning system (GPS) for the low Earth orbit (LEO) satellites. The global positioning system satellites fly in medium Earth orbits (MEO) at an altitude of approximately 20200 kilometers and they can provide unsurpassed observability to any low Earth orbit satellite. A LEO satellite is often able to track six or more GPS satellites with tracking arcs amounting to about half of its orbit. This ability along with high level redundancy of GPS satellites renders the GPS-based autonomous space navigation a robust method and naturally makes it a prime choice for LEO formation flying satellites. In the absence of GPS however, the autonomous space navigation issue for formation flying spacecrafts quickly becomes a daunting task specially for planetary exploration missions. Therefore we decided in this thesis to address the autonomous space navigation problem without utilizing 
at all the GPS. We will propose a very efficient relative orbit determination method for centralized-controlled close formation flying satellites and we will only utilize realtime observation data which are generated by an active phased array radar (APAR) mounted on the chief satellite.

The basis of formation flying is the dynamics of relative motion between two close satellites and any autonomous navigation system for close formation flying satellites requires a very accurate model of actual relative motion. A relative orbital motion model however, must be compact enough to allow its implementation on a typical space-qualified on-board computer. The most widely used relative motion model is known as the Clohessy-Wiltshire-Hill model $[3],[4],[5]$. This model provides a timeexplicit closed-form analytical solution to the relative motion problem for a circular unperturbed reference orbit. Lovell and Tragesser ${ }^{[6]}$ have further re-parametrized this model to demonstrate that the in-track and cross-track non-drifting relative motion about a circular unperturbed reference orbit always follows an elliptic trajectory. The Clohessy-Wiltshire-Hill model yields considerable errors when the eccentricity of the reference orbit increases ${ }^{[7]}$. Several models $[8],[9]$ have therefore been proposed to describe the relative motion about the elliptical reference orbits. Carter ${ }^{[10]}$, Lane and Axelrad ${ }^{[11]}$ developed time-explicit closed-form solutions for the relative motion of bounded elliptical reference orbits. Melton ${ }^{[12]}$ also proposed an alternative analytical solution for small eccentricity reference orbits. Some relative motion models also take into account orbit perturbations. The most important perturbation encountered for the relative motion problem and also the most extensively studied, is the perturbation caused by the oblateness of the Earth, referred to as $J_{2}$ gravitational perturbation. Sedwick and Schweighart ${ }^{[13],[14]}$ modified the classic Clohessy-Wiltshire-Hill model to include the orbital-averaged effect of the $J_{2}$ gravitational perturbation on a circular reference orbit. Kechichian ${ }^{[15]}$ developed a complete nonlinear description of relative motion for an elliptic reference orbit in the presence of $J_{2}$ gravitational perturbation. 
Unfortunately his system of equations can only be solved with numerical integration and It is clear that numerical methods are not well suited for autonomous on-board applications because they typically require a lot of computing effort and provide no insight into efficient guidance and control solutions. Gim and Alfriend ${ }^{[16]}$ devised a state transition matrix (STM) which provides a very accurate closed-form time explicit solution to the relative motion problem for a $J_{2}$ perturbed elliptical reference orbit. Gim and Alfriend state transition matrix is formulated using a geometric method in non-singular orbital elements and Brouwer theory ${ }^{[17]}$ to convert back and forth between mean and osculating differential orbit elements. Yan and Vadali [18] similarly developed a STM for a $J_{2}$ perturbed elliptic reference obit by projecting the motion of the chief and deputy satellites onto a unit sphere. Yan and Vadali method employs spherical trigonometry to create a kinematically exact description for the deputy satellite relative position vector in terms of the differential orbit elements. They obtained an analytical expression for the relative velocity vector by taking the time derivative of the relative position vector and with the help of the Gaussian form of the Lagrange planetary equations. Figure 1.1 depicts a classification of the major relative orbit motion theories. In this classification, the relative orbit motion models depending on the assumption they make on the level of eccentricity of the reference orbits are separated into three main categories and each category is further divided into two groups to indicate whether the relative orbit motion models take the $J_{2}$ gravitational perturbation into account or not.

Alfriend and Yan ${ }^{[19]}$ introduced a new modeling error index to evaluate and compare the accuracy of various theories of the satellites relative motion. Their study demonstrates that not including the reference orbit eccentricity and differential gravitational perturbations has a significant negative effect on the precision of the relative motion theory. Alfriend and Yan simulation results shows that the Yan and Vadali STM offers the most accurate solution followed closely by the Gim-Alfriend STM. 

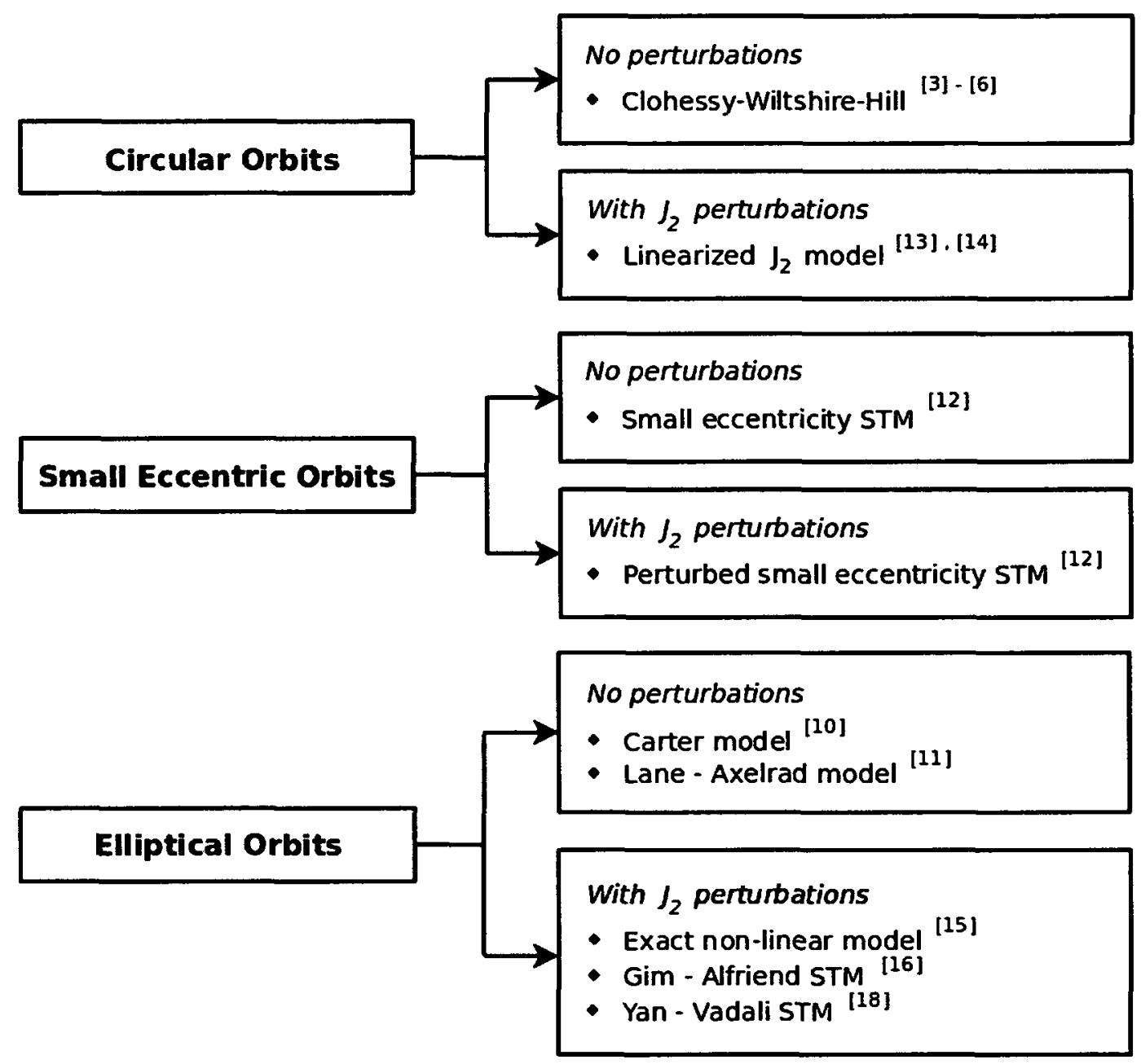

Figure 1.1: Relative motion theories classification

Nonetheless, in this thesis we utilize the Gim-Alfriend method for the following two reasons. First Gim-Alfriend STM provides directly a time explicit closed-form solution as opposed to Yan-Vadali STM. Yan-Vadali STM describes the relative motion in terms of the differential orbit elements and to find time-explicit expressions we need to employ Kepler's equation or apply eccentricity expansion. Second reason to use Gim-Alfriend STM is that it provides a linear model for the relative motion theory and therefore it is computationally more suitable for autonomous spacecrafts on-board applications. Yan-Vadali STM on other hand, provides an exact solution to the relative motion problem without recourse to any form of linearization and 
therefore it is computationally very intensive.

\subsection{Thesis Objective}

The purpose of this research is to develop an efficient and highly precise relative orbit determination method for Earth orbiting centralized-controlled autonomous close formation flying satellites. In centralized-controlled formation flight, one chief satellite is responsible for simultaneous navigation, guidance and control of all the remaining deputy satellites. Our proposed relative orbit determination method will employ a radar noisy observation data and iterated extended Kalman filter to estimate very accurately the relative orbit states of any deputy satellite flying in the formation.

\subsection{Thesis Overview}

Chapter 2 provides a thorough review of the general relative orbit description for the formation flying spacecrafts and develops in detail the Gim-Alfriend closed-form solution to the relative motion problem. This chapter also presents some elaborate simulation results to demonstrate the Gim-Alfriend STM performance for various close and far formation flying satellites.

Chapter 3 proposes a precise relative orbit determination method based on an iterated extended Kalman filter for close formation flying satellites. This method employs the Gim-Alfriend relative motion dynamics linear model and real-time noisy observation data collected by an active phased array radar mounted on the chief satellite to provide very accurate relative state estimates of the deputy satellites. An elegant and comprehensive set of simulation results exhibits the efficiency of our proposed relative orbit determination method.

Finally, chapter 4 presents a summary of simulation results, the conclusions of this research and recommendations for future work. 


\section{Chapter 2}

\section{Relative Orbit Description For Formation Flying Spacecraft}

In this chapter we develop thoroughly the relative orbit descriptions for two or more satellites that are flying in circular or elliptic inertial orbits. All satellite formations are assumed to consist of spacecrafts of equal structure and build and therefore the relative drag effects will have negligible influence on the relative orbit motion. The most prominent dynamical force studied in this chapter will be the gravitational attraction of planet Earth. We will examine in particular the effects of both a spherical and an oblate Earth on formation flying spacecrafts relative motions.

\subsection{General Relative Orbit Description}

The content of this section is mainly adopted from reference [20]. In this section, we develop the relative orbit equations of motion for a spherically symmetric Earth. Both the relative Cartesian coordinates and the orbit elements difference description will be considered. We begin with a general type of spacecraft formation flying geometry as shown in Figure 2.1. Here the two spacecrafts are on slightly different orbits that will fulfill specific constraints. These constraints make sure that the relative orbit remains bounded and these two spacecrafts will not gradually drift apart from the formation.

In any spacecrafts formation flight, the orbit of one satellite is considered to be 


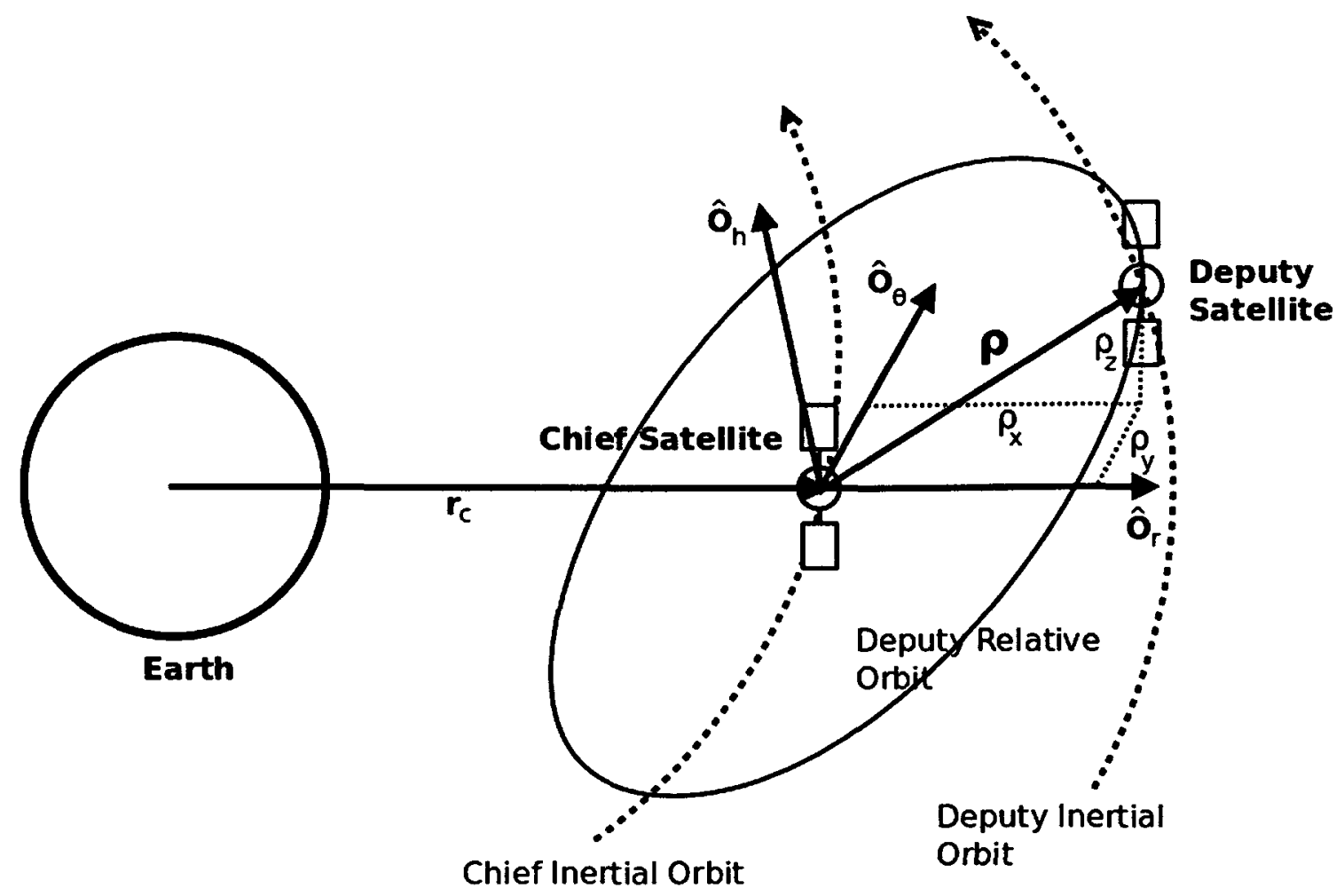

Figure 2.1: Illustration of a general type of two-spacecraft formation

a reference orbit for all other remaining satellites orbital motions. This reference satellite is often called chief satellite. The remaining satellites which are usually referred to as the deputy satellites fly in formation along with the chief satellite. We should note that it is not required that a physical satellite actually occupies the chief position. Occasionally, the chief position is soly utilized as an orbiting reference point about which all deputy satellites rotate.

The chief satellite inertial position is given by the vector $\boldsymbol{r}_{c}(t)$, and the deputy satellite position is expressed by $\boldsymbol{r}_{d}(t)$. We introduce the Hill Cartesian coordinate frame ${ }^{[3]}$ to exhibit how the relative orbit geometry is seen by the chief satellite. The Hill Cartesian coordinate frame origin is at the chief satellite position and the unit vector triad $\left\{\hat{\boldsymbol{o}}_{r}, \hat{\boldsymbol{o}}_{\theta}, \hat{\boldsymbol{o}}_{h}\right\}$ represents its orientation as depicted in Figure 2.1. The unit vector $\hat{\boldsymbol{o}}_{r}$ is in the orbit radius direction. The unit vector $\hat{\boldsymbol{o}}_{h}$ is parallel to 
the orbit angular momentum vector $\boldsymbol{h}$ which is perpendicular to the chief satellite orbital plane. The unit vector $\hat{\boldsymbol{o}}_{\theta}$ completes the right-hand coordinates system. The Hill Cartesian rotating reference frame is also referred to as the local-vertical-localhorizontal (LVLH) rectilinear frame. We denote the chief satellite LVLH rectilinear coordinate frame by symbol $\mathcal{O}$. Mathematically, the $\mathcal{O}$ frame orientation unit vectors are expressed as

$$
\begin{aligned}
\hat{\boldsymbol{o}}_{r} & =\frac{\boldsymbol{r}_{c}}{r_{c}} \\
\hat{\boldsymbol{o}}_{\theta} & =\hat{\boldsymbol{o}}_{h} \times \hat{\boldsymbol{o}}_{r} \\
\hat{\boldsymbol{o}}_{h} & =\frac{\boldsymbol{h}}{h}
\end{aligned}
$$

where $\boldsymbol{h}=\boldsymbol{r}_{c} \times \dot{\boldsymbol{r}}_{\boldsymbol{c}}$. We note that if the chief satellite inertial orbit is circular, then $\hat{\boldsymbol{o}}_{\theta}$ is parallel to the chief satellite velocity vector. The deputy satellite relative orbit position vector $\boldsymbol{\rho}$ is expressed in $\mathcal{O}$ frame components as

$$
\boldsymbol{\rho}=\left(\rho_{x}, \rho_{y}, \rho_{z}\right)^{T}
$$

\subsubsection{LVLH Rectilinear Frame Coordinate Description}

In this sub-section we describe the deputy satellite relative orbit motion in terms of the Cartesian coordinate vector $\boldsymbol{\rho}=\left(\rho_{x}, \rho_{y}, \rho_{z}\right)^{T}$. The vector $\boldsymbol{\rho}$ components are expressed in the chief satellite rotating LVLH rectilinear frame. The advantage of employing LVLH rectilinear frame coordinates is that the physical relative orbit dimensions are immediately evident from these Cartesian coordinates. The $\left(\rho_{x}, \rho_{y}\right)$ coordinates define the deputy satellite relative orbit motion in the chief satellite orbit plane. The $\rho_{z}$ coordinate specifies any deputy satellite motion out of the chief satellite orbit plane.

To obtain the deputy satellite relative equations of motion using Cartesian coor- 
dinates in the chief satellite rotating LVLH rectilinear frame, we express the deputy satellite position vector as

$$
\boldsymbol{r}_{d}=\boldsymbol{r}_{c}+\boldsymbol{\rho}=\left(r_{c}+\rho_{x}\right) \hat{\boldsymbol{o}}_{r}+\rho_{y} \hat{\boldsymbol{o}}_{\theta}+\rho_{z} \hat{\boldsymbol{o}}_{h}
$$

where $r_{c}$ is the orbit radius of the chief satellite. The angular velocity vector of the chief satellite rotating LVLH rectilinear frame $\mathcal{O}$ relative to the inertial frame $\mathcal{N}$ is given by

$$
\boldsymbol{\omega}_{\mathcal{O} / \mathcal{N}}=\dot{f} \hat{\boldsymbol{o}}_{h}
$$

where $f$ is the chief satellite LVLH rectilinear frame true anomaly. Taking two time derivatives of $\boldsymbol{r}_{d}$ with respect to the inertial frame $\mathcal{N}$ produces the deputy satellite acceleration vector as follows

$$
\begin{aligned}
\ddot{\boldsymbol{r}}_{d}=\left[\ddot{r}_{c}+\ddot{\rho}_{x}-2 \dot{\rho}_{y} \dot{f}-\ddot{f} \rho_{y}-\dot{f}^{2}\left(r_{c}+\rho_{x}\right)\right] \hat{\boldsymbol{o}}_{r} \\
\quad+\left[\ddot{\rho}_{y}+2 \dot{f}\left(\dot{r}_{c}+\dot{\rho}_{x}\right)+\ddot{f}\left(r_{c}+\rho_{x}\right)-\dot{f}^{2} \rho_{y}\right] \hat{\boldsymbol{o}}_{\theta}+\ddot{\rho}_{z} \hat{\boldsymbol{o}}_{h}
\end{aligned}
$$

We utilize the following identities to simplify the above kinematic expression. The chief satellite orbit angular momentum vector magnitude is given by $h=r_{c}^{2} \dot{f}$. Since $h$ is constant for Keplerian motion, taking the first time derivative of $h$ gives

$$
\dot{h}=0=2 r_{c} \dot{r}_{c} \dot{f}+r_{c}^{2} \ddot{f}
$$

Solving the above equation for the true anomaly acceleration yields

$$
\ddot{f}=-2 \frac{\dot{r}_{c}}{r_{c}} \dot{f}
$$

Moreover, we write the chief satellite position vector as $\boldsymbol{r}_{c}=r_{c} \hat{\boldsymbol{o}}_{r}$. Taking two time derivatives of $\boldsymbol{r}_{c}$ with respect to the inertial frame $\mathcal{N}$, the chief satellite acceleration 
vector is obtained by

$$
\ddot{\boldsymbol{r}}_{c}=\left(\ddot{r}_{c}-r_{c} \dot{f}^{2}\right) \hat{\boldsymbol{o}}_{r}
$$

On the other hand, the chief satellite orbit equations of motion is given by

$$
\ddot{\boldsymbol{r}}_{c}=-\frac{\mu}{r_{c}^{3}} \boldsymbol{r}_{c}=-\frac{\mu}{r_{c}^{2}} \hat{\boldsymbol{o}}_{r}
$$

Equating vector components in equations (2.8) and (2.9), the chief satellite orbit radius acceleration is stated as :

$$
\ddot{r}_{c}=r_{c} \dot{f}^{2}-\frac{\mu}{r_{c}^{2}}=r_{c} \dot{f}^{2}\left(1-\frac{\mu r_{c}}{\left(r_{c}^{2} \dot{f}\right)^{2}}\right)=r_{c} \dot{f}^{2}\left(1-\frac{r_{c}}{p}\right)
$$

where $p$ is the chief satellite orbit semiparameter. Now we substitute equations (2.7) and (2.10) into equation (2.5) to reduce the deputy satellite acceleration vector to

$$
\begin{aligned}
\ddot{\boldsymbol{r}}_{d}= & {\left[\ddot{\rho}_{x}-2 \dot{f}\left(\dot{\rho}_{y}-\rho_{y} \frac{\dot{r}_{c}}{r_{c}}\right)-\rho_{x} \dot{f}^{2}-\frac{\mu}{r_{c}^{2}}\right] \hat{\boldsymbol{o}}_{r} } \\
& +\left[\ddot{\rho}_{y}+2 \dot{f}\left(\dot{\rho}_{x}-\rho_{x} \frac{\dot{r}_{c}}{r_{c}}\right)-\rho_{y} \dot{f}^{2}\right] \hat{\boldsymbol{o}}_{\theta}+\ddot{\rho}_{z} \hat{\boldsymbol{o}}_{h}
\end{aligned}
$$

The deputy satellite orbital equations of motion expressed in the chief satellite LVLH rectilinear frame $\mathcal{O}$ is given by

$$
\ddot{\boldsymbol{r}}_{d}=-\frac{\mu}{r_{d}^{3}} \boldsymbol{r}_{d}=-\frac{\mu}{r_{d}^{3}}\left[\begin{array}{c}
r_{c}+\rho_{x} \\
\rho_{y} \\
\rho_{z}
\end{array}\right]
$$

where $r_{d}=\sqrt{\left(r_{c}+\rho_{x}\right)^{2}+\rho_{y}^{2}+\rho_{z}^{2}}$. Equating equations (2.11) and (2.12) yields the 
exact nonlinear relative equations of motion as follows :

$$
\begin{gathered}
\ddot{\rho}_{x}-2 \dot{f}\left(\dot{\rho}_{y}-\rho_{y} \frac{\dot{r}_{c}}{r_{c}}\right)-\rho_{x} \dot{f}^{2}-\frac{\mu}{r_{c}^{2}}=-\frac{\mu}{r_{d}^{3}}\left(r_{c}+\rho_{x}\right) \\
\ddot{\rho}_{y}+2 \dot{f}\left(\dot{\rho}_{x}-\rho_{x} \frac{\dot{r}_{c}}{r_{c}}\right)-\rho_{y} \dot{f}^{2}=-\frac{\mu}{r_{d}^{3}} \rho_{y} \\
\ddot{\rho}_{z}=-\frac{\mu}{r_{d}^{3}} \rho_{z}
\end{gathered}
$$

The only assumption that has been made to derive the above set of nonlinear relative equations of motion is that no perturbations are acting on the satellites and the orbital motion is simply Keplerian. In addition, the above relative equations of motion are acceptable for arbitrarily large relative orbits and the chief satellite orbit can be eccentric as well. If the deputy satellite relative orbit Cartesian coordinates $\left(\rho_{x}, \rho_{y}, \rho_{z}\right)$ are small in comparison with the chief satellite orbit radius $r_{c}$, then we can further simplify equations (2.13) as follows. The deputy satellite orbit radius $r_{d}$ can be approximated as

$$
r_{d}=r_{c} \sqrt{1+2 \frac{\rho_{x}}{r_{c}}+\frac{\rho_{x}^{2}+\rho_{y}^{2}+\rho_{z}^{2}}{r_{c}^{2}}} \approx r_{c} \sqrt{1+2 \frac{\rho_{x}}{r_{c}}}
$$

The above approximation allows us to write

$$
\frac{\mu}{r_{d}^{3}} \approx \frac{\mu}{r_{c}^{3}}\left(1+2 \frac{\rho_{x}}{r_{c}}\right)^{-3 / 2}
$$

Using binomial series expansion of $\left(1+2 \frac{\rho_{x}}{r_{c}}\right)^{-3 / 2}$ and neglecting $\frac{\rho_{x}}{r_{c}}$ terms with order higher than one we get

$$
\frac{\mu}{r_{d}^{3}} \approx \frac{\mu}{r_{c}^{3}}\left(1-3 \frac{\rho_{x}}{r_{c}}\right)
$$


The term $\mu / r_{c}^{3}$ can also be expressed in the following forms :

$$
\frac{\mu}{r_{c}^{3}}=\frac{h^{2}}{r_{c}^{3} p}=\frac{r_{c}^{4} \dot{f}^{2}}{r_{c}^{3} p}=\frac{r_{c}}{p} \dot{f}^{2}=\frac{\dot{f}^{2}}{1+e \cos f}
$$

We should note that the orbit elements shown in the above equation are the chief satellite orbit elements. We now simplify the right-hand side of equation (2.12) by applying approximation (2.16) to

$$
\begin{gathered}
-\frac{\mu}{r_{d}^{3}}\left[\begin{array}{c}
r_{c}+\rho_{x} \\
\rho_{y} \\
\rho_{z}
\end{array}\right] \approx-\frac{\mu}{r_{c}^{3}}\left(1-3 \frac{\rho_{x}}{r_{c}}\right)\left[\begin{array}{c}
r_{c}+\rho_{x} \\
\rho_{y} \\
\rho_{z}
\end{array}\right]=-\frac{\mu}{r_{c}^{3}}\left[\begin{array}{c}
r_{c}-2 \rho_{x}-\frac{3 \rho_{x}^{2}}{r_{c}} \\
\rho_{y}-\frac{3 \rho_{x} \rho_{y}}{r_{c}} \\
\rho_{z}-\frac{3 \rho_{x} \rho_{z}}{r_{c}}
\end{array}\right] \approx \\
-\frac{\mu}{r_{c}^{3}}\left[\begin{array}{c}
r_{c}-2 \rho_{x} \\
\rho_{y} \\
\rho_{z}
\end{array}\right]
\end{gathered}
$$

Substituting approximation (2.18) into equation (2.13) and simplifying the outcome expressions produces the deputy satellite relative orbit equations of motion, provided that $\rho_{x}, \rho_{y}, \rho_{z}$ are small in comparison with the chief satellite orbit radius $r_{c}$ :

$$
\begin{gathered}
\ddot{\rho}_{x}-\rho_{x} \dot{f}^{2}\left(1+2 \frac{r_{c}}{p}\right)-2 \dot{f}\left(\dot{\rho}_{y}-\rho_{y} \frac{\dot{r}_{c}}{r_{c}}\right)=0 \\
\ddot{\rho}_{y}+2 \dot{f}\left(\dot{\rho}_{x}-\rho_{x} \frac{\dot{r}_{c}}{r_{c}}\right)-\rho_{y} \dot{f}^{2}\left(1-\frac{r_{c}}{p}\right)=0 \\
\ddot{\rho}_{z}+\frac{r_{c}}{p} \dot{f}^{2} \rho_{z}=0
\end{gathered}
$$

We can use equations (2.17) and (2.7), along with the argument of latitude $\theta=\omega+f$ to write the deputy satellite general relative equations of motion in the following 
common form :

$$
\begin{gathered}
\ddot{\rho}_{x}-\rho_{x}\left(\dot{\theta}^{2}+2 \frac{\mu}{r_{c}^{3}}\right)-\rho_{y} \ddot{\theta}-2 \dot{\rho}_{y} \dot{\theta}=0 \\
\ddot{\rho}_{y}+2 \dot{\rho}_{x} \dot{\theta}+\rho_{x} \ddot{\theta}-\rho_{y}\left(\dot{\theta}^{2}-\frac{\mu}{r_{c}^{3}}\right)=0 \\
\ddot{\rho}_{z}+\frac{\mu}{r_{c}^{3}} \rho_{z}=0
\end{gathered}
$$

If we assume that the chief satellite orbit is circular, then $e=0, p=r_{c}$ and $r_{c}$ is constant. In addition, for a circular orbit the mean motion $n$ is equal to the true anomaly rate $\dot{f}$. Thus we can reduce the deputy satellite relative equations of motion to a much simpler form well-known as the Clohessy-Wiltshire (CW) equations :

$$
\begin{gathered}
\ddot{\rho}_{x}-2 n \dot{\rho}_{y}-3 n^{2} \rho_{x}=0 \\
\ddot{\rho}_{y}+2 n \dot{\rho}_{x}=0 \\
\ddot{\rho}_{z}+n^{2} \rho_{z}=0
\end{gathered}
$$

Note that the Clohessy-Wiltshire equations of motion are valid only if the chief satellite orbit is circular and the deputy satellite relative orbit Cartesian coordinates $\left(\rho_{x}, \rho_{y}, \rho_{z}\right)$ are small compared with the chief satellite orbit radius $r_{c}$. Moreover, the differential equations (2.21) has a simple form which allows them to be analytically integrated. A derivation of the closed-form solutions for the Clohessy-Wiltshire relative equations of motion is presented in reference [20] and therefore will not be repeated here. The resultant analytical solution in the chief satellite LVLH rectilinear 
frame is as follows :

$$
\begin{aligned}
& \rho_{x}(t)=A_{0} \cos (n t+\alpha)+\rho_{x_{\mathrm{off}}} \\
& \rho_{y}(t)=-2 A_{0} \sin (n t+\alpha)-\frac{3}{2} n t \rho_{x_{\mathrm{off}}}+\rho_{y_{\mathrm{off}}} \\
& \rho_{z}(t)=B_{0} \cos (n t+\beta)
\end{aligned}
$$

where $A_{0}, \alpha, B_{0}, \beta, \rho_{x_{\text {off }}}$, and $\rho_{y_{\text {off }}}$ are integration constants that are determined by the initial conditions. We notice that the expression for $\rho_{y}(t)$ contains a secular term that will increase infinitely large as $t \rightarrow \infty$. All the remaining terms in equations (2.22) are either sine or cosine functions, or constant offsets.

\subsubsection{Orbit Element Difference Description}

Although the LVLH rectilinear frame coordinates $\left(\rho_{x}, \rho_{y}, \rho_{z}\right)$ are widely used to describe a deputy satellite relative orbit, they have a major disadvantage that for a general relative orbit we need to solve a set of nonlinear differential equations first in order to acquire the deputy satellite precise relative orbit dimensions. In other words, because the deputy satellite relative orbit is determined through the chief satellite orbit motion and the deputy satellite relative orbit initial conditions

$$
\boldsymbol{X}_{\rho_{0}}=\left(\rho_{x_{0}}, \rho_{y_{0}}, \rho_{z_{0}}, \dot{\rho}_{x_{0}}, \dot{\rho}_{y_{0}}, \dot{\rho}_{z_{0}}\right)^{T}
$$

we need to integrate forward in time the differential equations (2.19) to find out where the deputy satellite would be at a time $t$ in future. This means that the six initial conditions in (2.23) which form six invariant quantities of the deputy satellite relative orbit, are not suitable to be utilized by the chief satellite on-board navigation software in order to determine the instantaneous geometry of the deputy satellite relative motion. Moreover, equations (2.19) are valid only if the deputy satellite 
relative orbit dimensions are small in comparison with the chief satellite orbit radius. Therefore instead of employing the six relative orbit invariants shown in (2.23) to specify a relative orbit for the deputy satellite, we would like to find other relative orbit invariant parameters that would produce equivalent results but their applications are not limited to those relative orbits that have very small dimensions.

To achieve this goal, we first review how the inertial orbit of a spacecraft is mathematically described in a Keplerian two-body motion and how we can solve the resultant equations of motion. Lets assume $\boldsymbol{r}$ represents the inertial position vector of a spacecraft which orbits a spherical planet. Then we must solve the following differential equations to determine the inertial position of this spacecraft

$$
\ddot{\boldsymbol{r}}=-\frac{\mu}{r^{3}} \boldsymbol{r}
$$

where the initial conditions are $\boldsymbol{r}\left(t_{0}\right)=\boldsymbol{r}_{0}$ and $\dot{\boldsymbol{r}}\left(t_{0}\right)=\dot{\boldsymbol{r}}_{0}$. These six initial conditions construct the six invariant parameters for the Keplerian two-body motion dynamical system. Alternatively, the Keplerian motion of a satellite can also be defined through six orbit elements. For example, we define the orbit element vector $e$ to include the following parameters

$$
\boldsymbol{e}=\left(a, e, i, \Omega, \omega, M_{0}\right)^{T}
$$

where $a$ is the semimajor axis, $e$ is the eccentricity, $i$ is the orbit inclination angle, $\Omega$ is the longitude of the ascending node, $\omega$ is the argument of the periapsis, and $M_{0}$ is the initial mean anomaly. For this case, instead of solving six differential equations to find the satellite current states, we only need to solve one algebraic Kepler's equation numerically to find the satellite current mean anomaly angle. This means that there is only one state $M$ that must be solved for to obtain the satellite inertial position. Further we note that even if disturbances such as gravitational perturbations or atmospheric drag are present, the orbit elements (2.25) will change 
very slowly in comparison with the $\boldsymbol{X}_{\rho}$ state vector. Therefore the orbit elements (2.25) significantly simplifies the satellite orbital description and computation.

Now assuming that no disturbances are present, we specify the deputy satellite relative orbit in terms of the orbit element difference vector $\delta e$ as follows

$$
\delta \boldsymbol{e}=\boldsymbol{e}_{d}-\boldsymbol{e}_{c}=\left(\delta a, \delta e, \delta i, \delta \Omega, \delta \omega, \delta M_{0}\right)^{T}
$$

where $\boldsymbol{e}_{d}$ and $\boldsymbol{e}_{c}$ are the deputy satellite and the chief satellite orbit element vectors respectively. Note that the above method for the relative orbit description is not restricted to use solely the classical orbit element set (2.25). Any complete alternative set of orbit elements can be used as well. Moreover, by having $\delta \boldsymbol{e}$ and $\boldsymbol{e}_{c}$, we can readily compute the deputy satellite relative position at any given time by solving only one algebraic Kepler's equation. Therefore in a similar manner to the inertial orbit description, we are able to evade having to solve a complex set of nonlinear differential equations. Finally, it is evident that the deputy satellite relative orbit description in equation (2.26) does not make any assumptions on how small the relative orbit dimensions must be in comparison with the chief satellite orbit radius.

\subsubsection{Linear Mapping between LVLH Rectilinear Frame Co- ordinates and Orbit Element Differences}

To map between the relative orbit state $\boldsymbol{X}_{\rho}$ and the orbit element differences $\delta \boldsymbol{e}$, the classical nonlinear transformation method between inertial coordinates and orbit elements can be utilized. However, if the deputy satellite relative orbit dimensions are small compared with the chief satellite orbit radius, then it is feasible to develop a direct linear transformation $A\left(\boldsymbol{e}_{c}\right)$ between $\boldsymbol{X}_{\rho}$ and $\delta \boldsymbol{e}$.

$$
\boldsymbol{X}_{\rho}=A\left(\boldsymbol{e}_{c}\right) \delta \boldsymbol{e}
$$


To circumvent some numerical difficulties associated with near-circular orbits, we use the following orbit element vector $e$

$$
e=\left(a, \theta, i, q_{1}, q_{2}, \Omega\right)^{T}
$$

where $\theta$ is the argument of latitude and $q_{1}$ and $q_{2}$ are defined by

$$
\begin{aligned}
& q_{1}=e \cos \omega \\
& q_{2}=e \sin \omega
\end{aligned}
$$

Let us now define the following three LVLH rectilinear coordinate systems. we allow $\mathcal{O}$ and $\mathcal{O}_{\mathcal{D}}$ represent the LVLH rectilinear frames of the chief and deputy satellites respectively and we also let $\mathcal{N}$ indicates the inertial frame. Then $[\mathcal{O N}]$ is a direction cosine matrix which maps any vector components in the inertial frame to its corresponding components in the chief LVLH rectilinear frame. To relate the deputy satellite orbit element difference vector $\delta \boldsymbol{e}$ to is associated LVLH rectilinear frame cartesian coordinate vector $\boldsymbol{X}_{\boldsymbol{\rho}}$, we express the deputy satellite inertial position vector $\boldsymbol{r}_{d}$ in terms of the chief and deputy LVLH rectilinear frames components as

$$
\begin{aligned}
\boldsymbol{r}_{d}^{\mathcal{O}} & =\left(r_{c}+\rho_{x}, \rho_{y}, \rho_{z}\right)^{T} \\
\boldsymbol{r}_{d}^{\mathcal{O}_{\mathcal{D}}} & =\left(r_{d}, 0,0\right)^{T}
\end{aligned}
$$

We can map the deputy satellite position vector $\boldsymbol{r}_{d}$ from the deputy LVLH rectilinear frame vector components to the chief LVLH rectilinear frame vector components using

$$
\boldsymbol{r}_{d}^{\mathcal{O}}=[\mathcal{O N}]\left[\mathcal{N \mathcal { O } _ { \mathcal { D } } ]} \boldsymbol{r}_{d}^{\mathcal{O}_{\mathcal{D}}}\right.
$$

The orientations of the $\mathcal{O}$ and $\mathcal{O}_{\mathcal{D}}$ LVLH rectilinear coordinate frames relative to 
the inertial $\mathcal{N}$ frame can be given through the $(3-1-3)$ rotations of Euler angle sets $\left\{\Omega_{c}, i_{c}, \theta_{c}\right\}$ and $\left\{\Omega_{d}, i_{d}, \theta_{d}\right\}$ respectively. Therefore the direction cosine matrices $[\mathcal{O N}]$ and $\left[\mathcal{O}_{\mathcal{D}} \mathcal{N}\right]$ are expressed by

$$
\begin{aligned}
{[\mathcal{O N}] } & =\left[\mathcal{R}_{3}\left(\theta_{c}\right)\right]\left[\mathcal{R}_{1}\left(i_{c}\right)\right]\left[\mathcal{R}_{3}\left(\Omega_{c}\right)\right] \\
{\left[\mathcal{O}_{\mathcal{D}} \mathcal{N}\right] } & =\left[\mathcal{R}_{3}\left(\theta_{d}\right)\right]\left[\mathcal{R}_{1}\left(i_{d}\right)\right]\left[\mathcal{R}_{3}\left(\Omega_{d}\right)\right]
\end{aligned}
$$

where the rotation matrices $[\mathcal{R}]$ are defined as

$$
\begin{aligned}
{\left[\mathcal{R}_{1}(\gamma)\right] } & =\left[\begin{array}{ccc}
1 & 0 & 0 \\
0 & \cos \gamma & \sin \gamma \\
0 & -\sin \gamma & \cos \gamma
\end{array}\right] \\
{\left[\mathcal{R}_{2}(\gamma)\right] } & =\left[\begin{array}{ccc}
\cos \gamma & 0 & -\sin \gamma \\
0 & 1 & 0 \\
\sin \gamma & 0 & \cos \gamma
\end{array}\right] \\
{\left[\mathcal{R}_{3}(\gamma)\right] } & =\left[\begin{array}{ccc}
\cos \gamma & \sin \gamma & 0 \\
-\sin \gamma & \cos \gamma & 0 \\
0 & 0 & 1
\end{array}\right]
\end{aligned}
$$

Now we take the first variation of $\left[\mathcal{N O}_{\mathcal{D}}\right]$ and $r_{d}$ about the chief satellite motion to obtain a first-order approximations

$$
\begin{aligned}
{\left[\mathcal{N O}_{\mathcal{D}}\right] } & \approx[\mathcal{N O}]+\delta[\mathcal{N O}] \\
r_{d} & \approx r_{c}+\delta r
\end{aligned}
$$


Equation (2.33) is then expanded and yields

$$
\boldsymbol{r}_{d}^{\mathcal{O}}=\left(\left[I_{3 \times 3}\right]+[\mathcal{O N}] \cdot \delta[\mathcal{N O}]\right)\left[\begin{array}{c}
r_{c}+\delta r \\
0 \\
0
\end{array}\right]
$$

We further simplify the above expression for the deputy satellite position vector by dropping the first variation terms of the second order. We also abandon using the subscript $c$ from here on and any parameter without a subscript is implied to be a chief satellite orbit parameter. Thus we can write

$r_{d}^{\mathcal{O}}=\left[\begin{array}{c}r+\delta r \\ 0 \\ 0\end{array}\right]+r[\mathcal{O N}]\left[\begin{array}{c}(-s \theta c \Omega-c \theta c i s \Omega) \delta \theta+(s \theta s i s \Omega) \delta i+(-c \theta s \Omega-s \theta c i c \Omega) \delta \Omega \\ (-s \theta s \Omega+c \theta c i c \Omega) \delta \theta-(s \theta s i c \Omega) \delta i+(c \theta c \Omega-s \theta c i s \Omega) \delta \Omega \\ (c \theta s i) \delta \theta+(s \theta c i) \delta i\end{array}\right]$

where the shorthand notations $s \gamma=\sin \gamma$ and $c \gamma=\cos \gamma$ are used. After some matrix algebra, the deputy position vector $\boldsymbol{r}_{d}^{\mathcal{O}}$ is partially expressed in terms of orbit elements and their differences by

$$
\boldsymbol{r}_{d}^{\mathcal{O}}=\left[\begin{array}{c}
r+\delta r \\
0 \\
0
\end{array}\right]+r\left[\begin{array}{c}
0 \\
\delta \theta+(\cos i) \delta \Omega \\
(\sin \theta) \delta i-(\cos \theta \sin i) \delta \Omega
\end{array}\right]
$$

To write completely $\boldsymbol{r}_{d}^{\mathcal{O}}$ in terms of the selected orbit elements and their differences, we must express the chief satellite orbit radius $r$ in terms of $\left(a, \theta, i, q_{1}, q_{2}, \Omega\right)$ orbit 
elements. Using identities (2.29) and (2.30) we have

$$
r=\frac{p}{1+e \cos f}=\frac{a\left(1-e^{2}\right)}{1+e \cos (\theta-\omega)}=\frac{a\left(1-q_{1}^{2}-q_{2}^{2}\right)}{1+q_{1} \cos \theta+q_{2} \sin \theta}
$$

Thus, the variation of $r$ is given by

$$
\delta r=\frac{r}{a} \delta a+\frac{V_{r}}{V_{t}} r \delta \theta-\frac{r}{p}\left(2 a q_{1}+r \cos \theta\right) \delta q_{1}-\frac{r}{p}\left(2 a q_{2}+r \sin \theta\right) \delta q_{2}
$$

where the chief satellite radial and tangential velocity components $V_{r}$ and $V_{t}$ are defined as

$$
\begin{aligned}
& V_{r}=\dot{r}=\frac{h}{p}\left(q_{1} \sin \theta-q_{2} \cos \theta\right) \\
& V_{t}=r \dot{\theta}=\frac{h}{p}\left(1+q_{1} \cos \theta+q_{2} \sin \theta\right)
\end{aligned}
$$

where $h$ is the chief satellite orbit angular momentum vector magnitude and $p$ is the chief satellite orbit semilatus rectum. Comparing the chief LVLH rectilinear frame components of the deputy satellite position vector $\boldsymbol{r}_{d}^{\mathcal{O}}$ in identities (2.31) and (2.40), we can express the Cartesian coordinates $\rho_{x}, \rho_{y}$, and $\rho_{z}$ in terms of the orbit element differences as :[21, 22]

$$
\begin{aligned}
& \rho_{x}=\delta r \\
& \rho_{y}=r[\delta \theta+(\cos i) \delta \Omega] \\
& \rho_{z}=r[(\sin \theta) \delta i-(\cos \theta \sin i) \delta \Omega]
\end{aligned}
$$

with $r$ and $\delta r$ are given by equations (2.41) and (2.42) respectively.

Up to this point, half of the desired transformations between the deputy satellite orbit element differences and the corresponding LVLH rectilinear frame Cartesian coordinates have been derived. We can further use an analogous approach to derive a 
linear relationship between the orbit element differences and the time rate of change of Cartesian coordinates $\left(\dot{\rho}_{x}, \dot{\rho}_{y}, \dot{\rho}_{z}\right)$. In reference [21], the deputy satellite velocity vector $\boldsymbol{v}_{d}$ is expressed in both the chief $\mathcal{O}$ and deputy $\mathcal{O}_{\mathcal{D}}$ LVLH rectilinear coordinate frames. Thus if we start from

$$
\boldsymbol{v}_{d}^{\mathcal{O}}=[\mathcal{O N}]\left[\mathcal{N O}_{\mathcal{D}}\right] \boldsymbol{v}_{d}^{\mathcal{O}_{\mathcal{D}}}
$$

and follow the similar steps as have been taken to derive equations (2.45), we will develop the deputy satellite desired Cartesian coordinate rates by comparing two algebraic expressions.

Alternatively, it is also possible to obtain the time rate of change of the deputy satellite Cartesian coordinates in terms of the orbit element differences by directly differentiating equations (2.45) with respect to time. We note that the only time varying quantities on the right hand side of equations (2.45) are $\theta$ and $\delta \theta$. Only the latter needs special treatment. We use the conservation law of angular momentum and write the following identity for the argument of latitude rate $\dot{\theta}$

$$
\dot{\theta}=\frac{h}{r^{2}}
$$

Then, the first variation of the above equation yields

$$
\delta \dot{\theta}=\frac{\delta h}{r^{2}}-2 \frac{h}{r^{3}} \delta r
$$

Utilizing the orbit angular momentum expression $h=\sqrt{\mu p}$, the $\delta h$ variation is given by

$$
\delta h=\frac{h}{2 p} \delta p
$$


where $\delta p$ is expressed as

$$
\delta p=\delta\left[a\left(1-e^{2}\right)\right]=\delta\left[a\left(1-q_{1}^{2}-q_{2}^{2}\right)\right]=\frac{p}{a} \delta a-2 a\left(q_{1} \delta q_{1}+q_{2} \delta q_{2}\right)
$$

Thus the first variation in the argument of latitude rate is

$$
\delta \dot{\theta}=\frac{h}{r^{2}}\left(\frac{\delta p}{2 p}-2 \frac{\delta r}{r}\right)
$$

After differentiating equations (2.45) and applying equation (2.51), the deputy satellite Cartesian coordinate rates in terms of orbit element differences are obtained as follows : $: 21,22]$

$$
\begin{aligned}
& \dot{\rho}_{x}=-\frac{V_{r}}{2 a} \delta a+\left(\frac{1}{r}-\frac{1}{p}\right) h \delta \theta+\left(V_{r} a q_{1}+h \sin \theta\right) \frac{\delta q_{1}}{p}+\left(V_{r} a q_{2}-h \cos \theta\right) \frac{\delta q_{2}}{p} \\
& \dot{\rho}_{y}=-\frac{3 V_{t}}{2 a} \delta a-V_{r} \delta \theta+\left(3 V_{t} a q_{1}+2 h \cos \theta\right) \frac{\delta q_{1}}{p}+\left(3 V_{t} a q_{2}+2 h \sin \theta\right) \frac{\delta q_{2}}{p}+V_{r} \cos i \delta \Omega \\
& \dot{\rho}_{z}=\left(V_{t} \cos \theta+V_{r} \sin \theta\right) \delta i+\left(V_{t} \sin \theta-V_{r} \cos \theta\right) \sin i \delta \Omega
\end{aligned}
$$

Equations (2.45) and (2.52) together define a linear mapping $A\left(\boldsymbol{e}_{c}\right)$ from the orbit element differences $\delta \boldsymbol{e}$ to the corresponding relative orbit states $\boldsymbol{X}_{\boldsymbol{\rho}}$.

\subsection{Relative Motion State Transition Matrix for the perturbed Chief Orbit}

The intent of this section is to derive a precise analytic solution for the relative orbit motion of a deputy satellite that includes the effects of the chief orbit eccentricity and the gravitational perturbation $J_{2}$. We apply a geometric method which uses the relationship between the relative orbit states and the orbit elements differences to obtain a state transition matrix in closed form. ${ }^{[16]}$ This state transition matrix which was originally developed by professor Kyle T. Alfriend and his research associate Dr. 
Dong-Woo Gim in Texas A\&M university, alleviates the need to directly solve a set of complex relative orbit motion differential equations that have the $J_{2}$ zonal harmonic perturbation terms.

We define the orbital elements of the chief satellite and the relative position and velocity vectors of the deputy satellite as $\boldsymbol{e}=\left(a, \theta, i, q_{1}, q_{2}, \Omega\right)^{T}$ and $\boldsymbol{X}=$ $(x, \dot{x}, y, \dot{y}, z, \dot{z})^{T}$, where $\theta$ is the argument of latitude, $q_{1}=e \cos \omega$, and $q_{2}=e \sin \omega$. The orbital element set $\boldsymbol{e}$ is used because the true anomaly $f$ and the argument of periapsis $\omega$ are undefined for a circular orbit and there exist also numerical problems in the eccentricity $e$ mean to osculating transformation for near-circular orbits. Moreover, It is clear that because the longitude of the ascending node $\Omega$ is undefined for the inclination $i=0$ degree, the Gim-Alfriend analytical solution for the relative orbit motion can not be applied to equatorial chief and deputy satellites orbits. After finding the orbit elements of the deputy satellite by applying a Taylor-series expansion about the orbit elements of the chief satellite, the relative orbit elements between the deputy and chief are expressed by $\delta \boldsymbol{e}=\boldsymbol{e}_{d}-\boldsymbol{e}_{c}$. For notational simplification, we assume that the orbit elements $\boldsymbol{e}$ without subscript describe the chief inertial motion, and $\boldsymbol{X}$ and $\delta \boldsymbol{e}$ describe the deputy satellite relative motion. We will use a local vertical local horizontal (LVLH) curvilinear coordinate system $O$ represented by unit vectors $\left(\hat{\boldsymbol{e}}_{x}, \hat{\boldsymbol{e}}_{y}, \hat{\boldsymbol{e}}_{z}\right)$ instead of the LVLH rectilinear frame $\mathcal{O}$ to obtain more accurate results. The curvilinear coordinate system $O$ origin is at the chief satellite position and $x$ is a difference in the radii and $y$ and $z$ are the curvilinear distances along the imaginary circular orbits on and perpendicular to the chief orbital plane respectively as shown in Figure 2.2.

\subsubsection{Gim-Alfriend Geometric Method}

Employing the osculating orbit elements $e$ and $e_{d}$ for the chief and deputy satellites respectively under the influence of $J_{2}$ gravitational disturbances and also using the instantaneous total angular velocity $\varpi$ of the chief satellite LVLH curvilinear coor- 


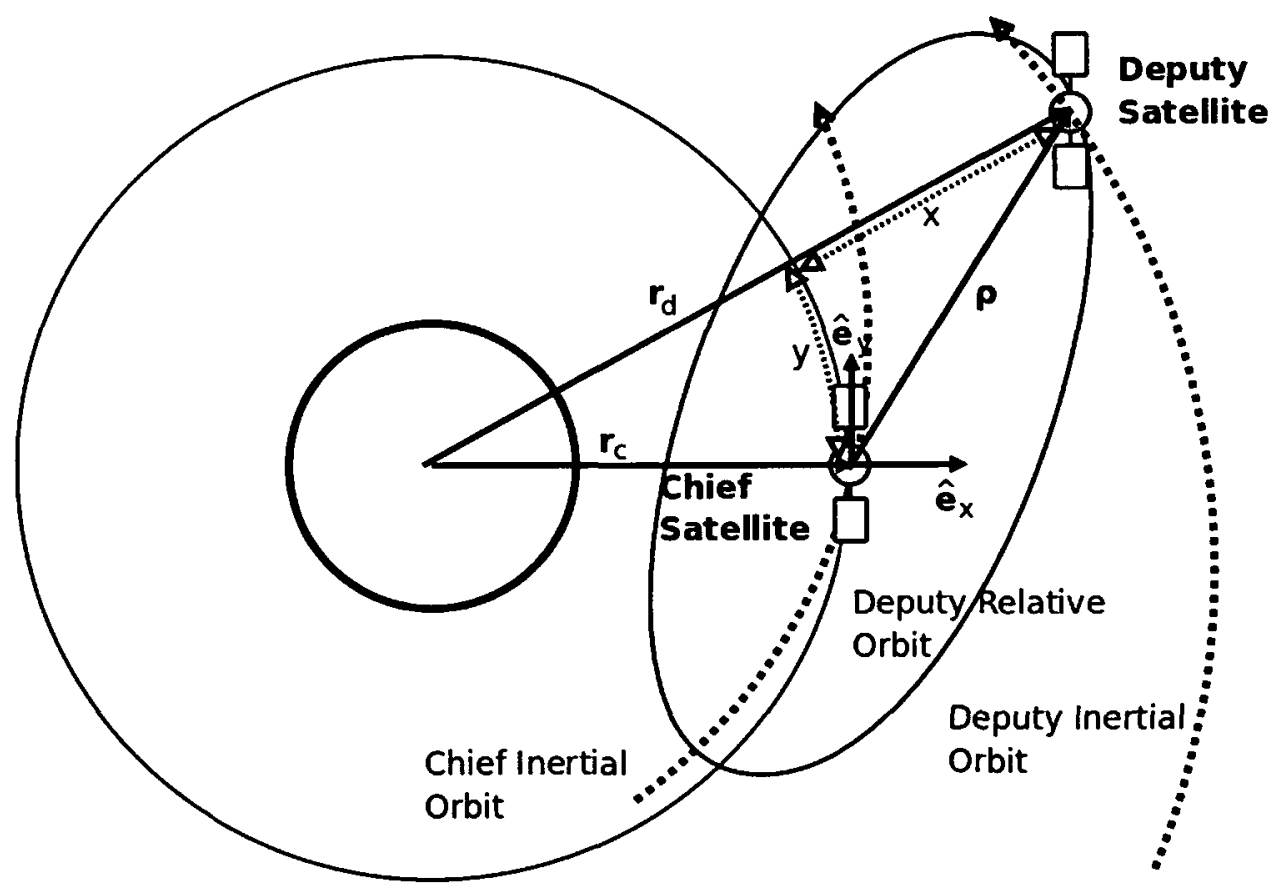

Figure 2.2: Illustration of a general type of spacecraft formation in the chief LVLH curvilinear coordinate system

dinate system $O$ with respect to the Earth Centered Inertial (ECI) reference frame, we can represent the geometric transformation between $\boldsymbol{X}(t)$ and $\delta \boldsymbol{e}(t)$ by

$$
\boldsymbol{X}(t)=[A(t)+\sigma B(t)] \delta \boldsymbol{e}(t)
$$

where $\sigma=3 J_{2} R_{e}^{2}, R_{e}$ is the Earth equatorial radius, and matrix $B(t)$ contains only terms that are perturbed by $J_{2}$ disturbances. We let $\Phi_{\delta \boldsymbol{e}}\left(t, t_{0}\right)$ be the state transition matrix for the relative osculating orbit elements, i.e.

$$
\delta e(t)=\Phi_{\delta e}\left(t, t_{0}\right) \delta e\left(t_{0}\right)
$$

Thus, from the solution

$$
\boldsymbol{X}(t)=\Phi_{\boldsymbol{X}}\left(t, t_{0}\right) \boldsymbol{X}\left(t_{0}\right)
$$


we can express the state transition matrix $\Phi_{\boldsymbol{X}}\left(t, t_{0}\right)$ for the relative instantaneous motion by

$$
\Phi_{\mathbf{X}}\left(t, t_{0}\right)=[A(t)+\sigma B(t)] \Phi_{\delta \boldsymbol{e}}\left(t, t_{0}\right)\left[A\left(t_{0}\right)+\sigma B\left(t_{0}\right)\right]^{-1}
$$

We further utilize a transformation matrix $D(t)$ to map the relative mean orbit elements $\delta \overline{\boldsymbol{e}}$ to the relative osculating orbit elements $\delta \boldsymbol{e}$

$$
D(t)=\frac{\delta \boldsymbol{e}_{\mathrm{osc}}(t)}{\delta \boldsymbol{e}_{\mathrm{mean}}(t)}=\frac{\delta \boldsymbol{e}(t)}{\delta \overline{\boldsymbol{e}}(t)}
$$

We also let $\Phi_{\delta \bar{e}}\left(t, t_{0}\right)$ be the state transition matrix for the relative mean orbit elements

$$
\delta \overline{\boldsymbol{e}}(t)=\Phi_{\delta \bar{e}}\left(t, t_{0}\right) \delta \overline{\boldsymbol{e}}\left(t_{0}\right)
$$

and from equation (2.57) we write

$$
\delta \boldsymbol{e}(t)=D(t) \delta \overline{\boldsymbol{e}}(t)=D(t) \Phi_{\delta \overline{\boldsymbol{e}}}\left(t, t_{0}\right) \delta \overline{\boldsymbol{e}}\left(t_{0}\right)=D(t) \Phi_{\delta \overline{\boldsymbol{e}}}\left(t, t_{0}\right) D^{-1}\left(t_{0}\right) \delta \boldsymbol{e}\left(t_{0}\right)
$$

Thus using identity (2.54), we can relate the state transition matrix for the relative osculating orbit elements to the state transition matrix for the relative mean orbit elements by

$$
\Phi_{\delta e}\left(t, t_{0}\right)=D(t) \Phi_{\delta \bar{e}}\left(t, t_{0}\right) D^{-1}\left(t_{0}\right)
$$

and subsequently the state transition matrix $\Phi_{\boldsymbol{X}}\left(t, t_{0}\right)$ takes the following form

$$
\Phi_{\boldsymbol{X}}\left(t, t_{0}\right)=[A(t)+\sigma B(t)] D(t) \Phi_{\delta \bar{e}}\left(t, t_{0}\right) D^{-1}\left(t_{0}\right)\left[A\left(t_{0}\right)+\sigma B\left(t_{0}\right)\right]^{-1}
$$


If we employ the mean orbit elements $\bar{e}$ and $\bar{e}_{d}$ for the chief and deputy satellites respectively under the influence of $J_{2}$ gravitational disturbances and also use the mean total angular velocity $\bar{\varpi}$ of the LVLH curvilinear coordinate system $O$ with respect to the ECI reference frame, we can represent the geometric transformation between the relative mean state $\boldsymbol{X}_{\text {mean }}(t) \equiv \overline{\boldsymbol{X}}(t)$ and the relative mean orbit elements $\delta \boldsymbol{e}_{\text {mean }}(t) \equiv$ $\delta \overline{\boldsymbol{e}}(t)$ by the following equation. Note that $\overline{\boldsymbol{X}}$ only represents the secular part of the deputy satellite relative instantaneous motion.

$$
\overline{\boldsymbol{X}}(t)=[\bar{A}(t)+\sigma \bar{B}(t)] \delta \overline{\boldsymbol{e}}(t)
$$

Similarly, from identity (2.58) and the solution

$$
\overline{\boldsymbol{X}}(t)=\Phi_{\overline{\boldsymbol{X}}}\left(t, t_{0}\right) \overline{\boldsymbol{X}}\left(t_{0}\right)
$$

the state transition matrix for the relative mean motion becomes

$$
\Phi_{\overline{\mathbf{X}}}\left(t, t_{0}\right)=[\bar{A}(t)+\sigma \bar{B}(t)] \Phi_{\delta \bar{e}}\left(t, t_{0}\right)\left[\bar{A}\left(t_{0}\right)+\sigma \bar{B}\left(t_{0}\right)\right]^{-1}
$$

We note that when there is no $J_{2}$ gravitational disturbances, the relative mean and osculating states are equal, i.e. $\overline{\boldsymbol{X}}(t)=\boldsymbol{X}(t)$. Additionally for $J_{2}=0$, the relative mean and osculating orbit elements are equal as well, $\delta \overline{\boldsymbol{e}}(t)=\delta \boldsymbol{e}(t)$. Thus from identities (2.53) and (2.62), we conclude that $\bar{A}(t)=A(t)$ if $J_{2}=0$. On the other hand, when $J_{2} \neq 0$, the mean total angular velocity $\bar{\varpi}$ and the instantaneous total angular velocity $\varpi$ of the LVLH curvilinear coordinate system $O$ with respect to the ECI reference frame are different. Therefore we can assert that $\bar{B}(t) \neq B(t)$. 


\subsubsection{Instantaneous Transformation matrix $\mathcal{T}$}

We now determine the instantaneous transformation matrix $\mathcal{T} \equiv[A(t)+\sigma B(t)]$. We start by finding an expression for the instantaneous total angular velocity $\varpi$ of the chief satellite. We assume that the chief satellite body frame axes are always aligned with the chief satellite LVLH curvilinear frame axes. We employ the longitude of the ascending node angle $\Omega$, the inclination angle $i$, and the argument of latitude angle $\theta$ to describe the orientation of the chief satellite LVLH curvilinear coordinate system $O$ relative to the ECI reference frame. To demonstrate how these Euler's angles $\Omega, i$, and $\theta$, in sequence, can provide a description of the chief satellite coordinate system $O$ orientation, we shall follow a body-axes triad as it assumes different orientations in the inertial space. Figure 2.3 displays three consecutive rotations through angles $\Omega, i$, and $\theta$ of a body-axes triad. This body-axes triad is initially coincident with the ECI reference frame axes $X, Y$, and $Z$. A rotation through angle $\Omega$ about axis $Z$ brings the triad into coincidence with axes $\xi^{\prime \prime}, \eta^{\prime \prime}, \zeta^{\prime \prime}$. A further rotation $i$ about axis $\xi^{\prime \prime}$ puts the triad into orientation $\xi^{\prime}, \eta^{\prime}, \zeta^{\prime}$ where axis $\xi^{\prime}$, often referred to as the nodal axis remains in the Earth's equatorial plane at all times. Finally, a rotation $\theta$ about axis $\zeta^{\prime}$ makes the triad coincide with axes $\xi, \eta, \zeta$ which are parallel to the chief satellite LVLH curvilinear coordinate system $O$ unit vectors $\hat{e}_{x}, \hat{\boldsymbol{e}}_{y}$, and $\hat{\boldsymbol{e}}_{z}$ respectively. Lets concentrate on the node-axis coordinate system $\xi^{\prime}, \eta^{\prime}, \zeta^{\prime}$. It is easy to see from Figure 2.3 that the angular velocity of the node-axis coordinate system $\xi^{\prime}, \eta^{\prime}, \zeta^{\prime}$ is

$$
\boldsymbol{\omega}=\left[\begin{array}{c}
\omega_{\xi^{\prime}} \\
\omega_{\eta^{\prime}} \\
\omega_{\zeta^{\prime}}
\end{array}\right]=\left[\begin{array}{c}
\dot{i} \\
\dot{\Omega} \sin i \\
\dot{\Omega} \cos i
\end{array}\right]
$$

Further, we learned that the coordinate system $\xi, \eta, \zeta$ is obtained by rotating the node-axis coordinate system $\xi^{\prime}, \eta^{\prime}, \zeta^{\prime}$ through angle $\theta$ about axis $\zeta^{\prime}$. Thus the in- 


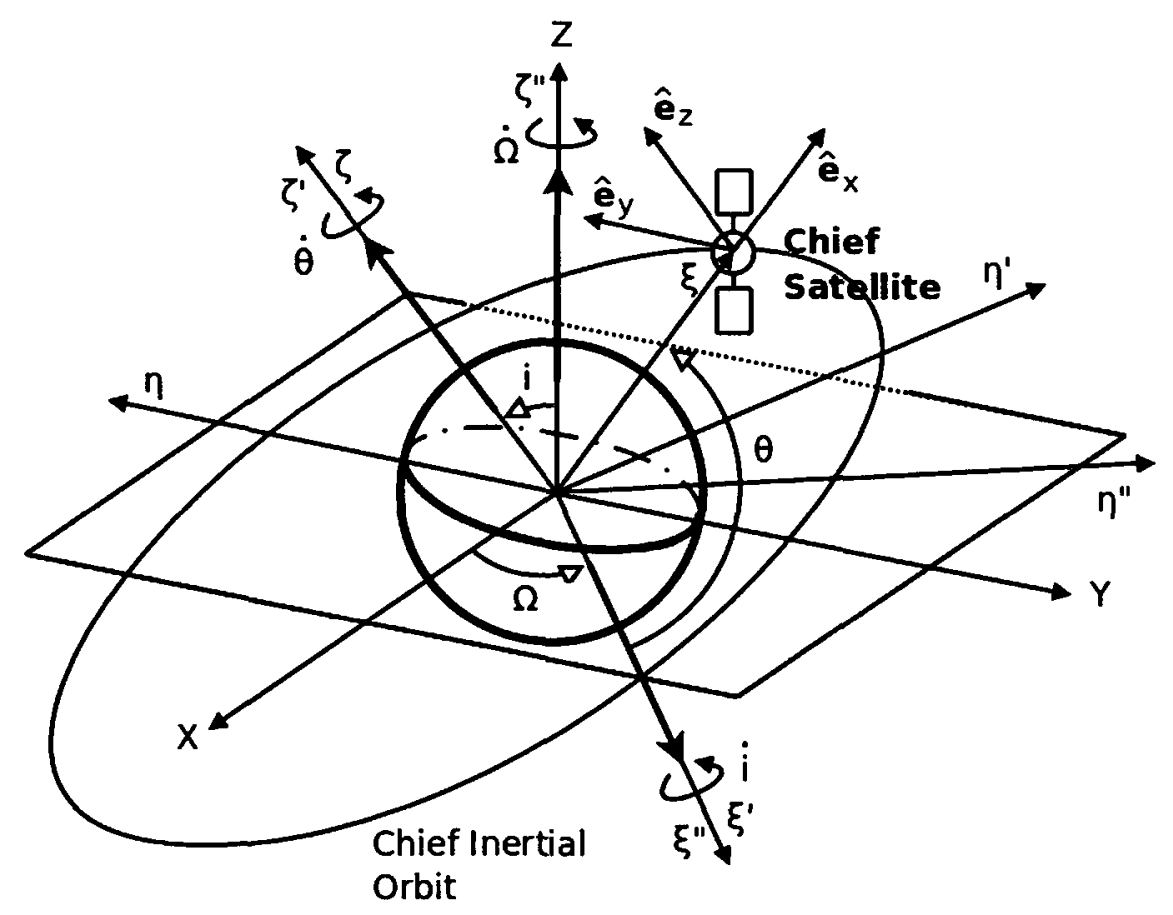

Figure 2.3: Illustration of three consecutive rotations through Euler's angles $\Omega, i$, and $\theta$ of a body-axes triad

stantaneous total angular velocity $\varpi$ of the chief satellite can be expressed in the coordinate system $\xi, \eta, \zeta$ by

$$
\varpi=\left[\begin{array}{c}
\varpi_{\xi} \\
\varpi_{\eta} \\
\varpi_{\zeta}
\end{array}\right]=\left[\begin{array}{ccc}
\cos \theta & \sin \theta & 0 \\
-\sin \theta & \cos \theta & 0 \\
0 & 0 & 1
\end{array}\right]\left[\begin{array}{c}
\dot{\Omega} \\
\dot{\Omega} \sin i \\
\dot{\Omega} \cos i
\end{array}\right]+\left[\begin{array}{c}
0 \\
0 \\
\dot{\theta}
\end{array}\right]
$$

Since coordinate axes $\xi, \eta$, and $\zeta$ are parallel to the chief satellite LVLH curvilinear coordinate system $O$ unit vectors $\hat{\boldsymbol{e}}_{x}, \hat{\boldsymbol{e}}_{y}$, and $\hat{\boldsymbol{e}}_{z}$ respectively, we convert the above matrix expression for the instantaneous total angular velocity $\varpi$ into the following vector form

$$
\varpi=\varpi_{r} \hat{\boldsymbol{e}}_{x}+\varpi_{t} \hat{\boldsymbol{e}}_{y}+\varpi_{n} \hat{\boldsymbol{e}}_{z}
$$


where $\varpi_{r}, \varpi_{t}$, and $\varpi_{n}$ are the vector components of $\varpi$ along the chief satellite LVLH curvilinear coordinate system $O$ unit vectors.

$$
\begin{aligned}
& \varpi_{r}=\dot{i} \cos \theta+\dot{\Omega} \sin \theta \sin i \\
& \varpi_{t}=-\dot{i} \sin \theta+\dot{\Omega} \cos \theta \sin i \\
& \varpi_{n}=\dot{\Omega} \cos i+\dot{\theta}
\end{aligned}
$$

Now we turn our focus on finding matrices $A(t)$ and $B(t)$. The position and velocity vectors of the chief satellite are

$$
\begin{aligned}
& \boldsymbol{r}=r \hat{\boldsymbol{e}}_{x} \\
& \boldsymbol{v}=\dot{r} \hat{\boldsymbol{e}}_{x}+r \varpi_{n} \hat{\boldsymbol{e}}_{y}-r \varpi_{t} \hat{\boldsymbol{e}}_{z} \equiv V_{r J_{2}} \hat{\boldsymbol{e}}_{x}+V_{t J_{2}} \hat{\boldsymbol{e}}_{y}+V_{n J_{2}} \hat{\boldsymbol{e}}_{z}
\end{aligned}
$$

Similarly, the position and velocity vectors of the deputy satellite are expressed by

$$
\begin{aligned}
\boldsymbol{r}_{d}= & \boldsymbol{r}+\boldsymbol{\rho}=(r+x) \hat{\boldsymbol{e}}_{x}+y \hat{\boldsymbol{e}}_{y}+z \hat{\boldsymbol{e}}_{z} \\
\boldsymbol{v}_{d}= & \left(V_{r J_{2}}+\dot{x}-y \varpi_{n}+z \varpi_{t}\right) \hat{\boldsymbol{e}}_{x}+\left(V_{t J_{2}}+\dot{y}+x \varpi_{n}-z \varpi_{r}\right) \hat{\boldsymbol{e}}_{y}+ \\
& \left(V_{n J_{2}}+\dot{z}-x \varpi_{t}+y \varpi_{r}\right) \hat{\boldsymbol{e}}_{z}
\end{aligned}
$$

We further obtain a first-order approximations of the deputy satellite position and velocity vectors in the chief LVLH curvilinear coordinate system $O$ by applying Taylor series expansions about the chief motion. We will follow the analogous steps as in subsection 2.1.3. We let $O$ and $O_{\mathcal{D}}$ represent the LVLH curvilinear coordinate systems of the chief and the deputy satellites respectively and we also let $\mathcal{N}$ denote the ECI reference frame. Thus $[O \mathcal{N}]$ will be a direction cosine matrix mapping any vector components in the inertial frame to its components in the chief LVLH curvilinear frame. We express the deputy satellite inertial position vector $\boldsymbol{r}_{d}$ in terms of the chief 
and deputy LVLH curvilinear frames components as

$$
\begin{aligned}
\boldsymbol{r}_{d}^{O} & =(r+x, y, z)^{T} \\
\boldsymbol{r}_{d}^{O_{\mathcal{D}}} & =\left(r_{d}, 0,0\right)^{T}
\end{aligned}
$$

Next, we map the deputy position vector $\boldsymbol{r}_{d}$ from the deputy LVLH curvilinear frame vector components to the chief LVLH curvilinear frame vector components.

$$
\boldsymbol{r}_{d}^{O}=[O \mathcal{N}]\left[\mathcal{N} O_{\mathcal{D}}\right] \boldsymbol{r}_{d}^{O_{\mathcal{D}}}
$$

The orientations of the $O$ and $O_{\mathcal{D}}$ curvilinear coordinate frames relative to the inertial $\mathcal{N}$ frame can be given through the $(3-1-3)$ rotations of Euler angle sets $\{\Omega, i, \theta\}$ and $\left\{\Omega_{d}, i_{d}, \theta_{d}\right\}$ respectively. Therefore the direction cosine matrices $[O \mathcal{N}]$ and $\left[O_{\mathcal{D}} \mathcal{N}\right]$ are expressed by

$$
\begin{gathered}
{[O \mathcal{N}]=\left[\mathcal{R}_{3}(\theta)\right]\left[\mathcal{R}_{1}(i)\right]\left[\mathcal{R}_{3}(\Omega)\right]} \\
{\left[O_{\mathcal{D}} \mathcal{N}\right]=\left[\mathcal{R}_{3}\left(\theta_{d}\right)\right]\left[\mathcal{R}_{1}\left(i_{d}\right)\right]\left[\mathcal{R}_{3}\left(\Omega_{d}\right)\right]}
\end{gathered}
$$

where the rotation matrices $[\mathcal{R}]$ are defined by identities $(2.35)$. Now we take the first variation of $\left[\mathcal{N} O_{\mathcal{D}}\right]$ and $r_{d}$ about the chief satellite motion to obtain a first-order approximations

$$
\begin{aligned}
{\left[\mathcal{N} O_{\mathcal{D}}\right] } & \approx[\mathcal{N} O]+\delta[\mathcal{N} O] \\
r_{d} & \approx r+\delta r
\end{aligned}
$$


The matrices $[\mathcal{N} O]$ and $\delta[\mathcal{N} O]$ are expressed by

$$
\begin{aligned}
& {[\mathcal{N} O]=} {\left[\begin{array}{ccc}
c \theta c \Omega-s \theta c i s \Omega & -s \theta c \Omega-c \theta c i s \Omega & s i s \Omega \\
c \theta s \Omega+s \theta c i c \Omega & -s \theta s \Omega+c \theta c i c \Omega & -s i c \Omega \\
s \theta s i & c \theta s i & c i
\end{array}\right] } \\
& \delta[\mathcal{N} O]=\left[\begin{array}{ccc}
\delta(\mathcal{N O})_{11} & \delta(\mathcal{N} O)_{12} & \delta(\mathcal{N} O)_{13} \\
\delta(\mathcal{N O})_{21} & \delta(\mathcal{N O})_{22} & \delta(\mathcal{N} O)_{23} \\
\delta(\mathcal{N O})_{31} & \delta(\mathcal{N O})_{32} & \delta(\mathcal{N O} O)_{33}
\end{array}\right]
\end{aligned}
$$

where $s \gamma=\sin \gamma, c \gamma=\cos \gamma$, and

$$
\begin{aligned}
& \delta(\mathcal{N} O)_{11}=(-s \theta c \Omega-c \theta c i s \Omega) \delta \theta+(s \theta s i s \Omega) \delta i+(-c \theta s \Omega-s \theta c i c \Omega) \delta \Omega \\
& \delta(\mathcal{N} O)_{12}=(-c \theta c \Omega+s \theta c i s \Omega) \delta \theta+(c \theta s i s \Omega) \delta i+(s \theta s \Omega-c \theta c i c \Omega) \delta \Omega \\
& \delta(\mathcal{N} O)_{13}=(c i s \Omega) \delta i+(s i c \Omega) \delta \Omega \\
& \delta(\mathcal{N} O)_{21}=(-s \theta s \Omega+c \theta c i c \Omega) \delta \theta+(-s \theta s i c \Omega) \delta i+(c \theta c \Omega-s \theta c i s \Omega) \delta \Omega \\
& \delta(\mathcal{N} O)_{22}=(-c \theta s \Omega-s \theta c i c \Omega) \delta \theta+(-c \theta s i c \Omega) \delta i+(-s \theta c \Omega-c \theta c i s \Omega) \delta \Omega \\
& \delta(\mathcal{N} O)_{23}=(-c i c \Omega) \delta i+(s i s \Omega) \delta \Omega \\
& \delta(\mathcal{N} O)_{31}=(c \theta s i) \delta \theta+(s \theta c i) \delta i \\
& \delta(\mathcal{N} O)_{32}=(-s \theta s i) \delta \theta+(c \theta c i) \delta i \\
& \delta(\mathcal{N} O)_{33}=(-s i) \delta i
\end{aligned}
$$


Substituting the first-order approximations of $\left[\mathcal{N} O_{\mathcal{D}}\right]$ and $r_{d}$ into equation (2.75) yields

$$
\begin{aligned}
\boldsymbol{r}_{d}^{O} & \approx[O \mathcal{N}]\{[\mathcal{N} O]+\delta[\mathcal{N} O]\}\left[\begin{array}{c}
r+\delta r \\
0 \\
0
\end{array}\right] \\
& \approx\left[\begin{array}{c}
r+\delta r \\
0 \\
0
\end{array}\right]+[O \mathcal{N}] \cdot \delta[\mathcal{N} O]\left[\begin{array}{c}
r+\delta r \\
0 \\
0
\end{array}\right]
\end{aligned}
$$

We further simplify the above approximation by discarding all the first variation terms of the second order. After some matrix algebra, the following expression for the deputy satellite position vector is obtained.

$$
\boldsymbol{r}_{d}^{O}=\left[\begin{array}{c}
r+\delta r \\
0 \\
0
\end{array}\right]+r\left[\begin{array}{c}
0 \\
\delta \theta+(c i) \delta \Omega \\
(s \theta) \delta i-(c \theta s i) \delta \Omega
\end{array}\right]
$$

Similarly, we map the deputy velocity vector $\boldsymbol{v}_{d}$ from the deputy LVLH curvilinear frame vector components to the chief LVLH curvilinear frame vector components.

$$
\boldsymbol{v}_{d}^{O}=[O \mathcal{N}]\left[\mathcal{N} O_{\mathcal{D}}\right] \boldsymbol{v}_{d}^{O_{\mathcal{D}}}
$$

We take the first variation of $v_{d}^{O_{\mathcal{D}}}$ about the chief satellite motion to obtain

$$
\boldsymbol{v}_{\boldsymbol{d}}^{O_{\mathcal{D}}} \approx \boldsymbol{v}+\delta \boldsymbol{v}
$$


Substituting the first-order approximations of $\left[\mathcal{N} O_{\mathcal{D}}\right]$ and $\boldsymbol{v}_{\boldsymbol{d}}^{O_{\mathcal{D}}}$ from equations $(2.77)$ and (2.85) into equation (2.84) results in

$$
\begin{gathered}
\boldsymbol{v}_{d}^{O} \approx[O \mathcal{N}]\{[\mathcal{N O} 0]+\delta[\mathcal{N O}]\}\left[\begin{array}{c}
V_{r J_{2}}+\delta V_{r J_{2}} \\
V_{t J_{2}}+\delta V_{t J_{2}} \\
V_{n J_{2}}+\delta V_{n J_{2}}
\end{array}\right]=\left[\begin{array}{c}
V_{r J_{2}}+\delta V_{r J_{2}} \\
V_{t J_{2}}+\delta V_{t J_{2}} \\
V_{n J_{2}}+\delta V_{n J_{2}}
\end{array}\right]+ \\
{[O \mathcal{N}] \cdot \delta[\mathcal{N} O]\left\{\left[\begin{array}{c}
V_{r J_{2}}+\delta V_{r J_{2}} \\
0 \\
0
\end{array}\right]+\left[\begin{array}{c}
0 \\
V_{t J_{2}}+\delta V_{t J_{2}} \\
0
\end{array}\right]+\left[\begin{array}{c}
0 \\
0 \\
V_{n J_{2}}+\delta V_{n J_{2}}
\end{array}\right]\right\}}
\end{gathered}
$$

We delete all the first variation terms of the second order to further refine the above approximation. After conducting some lengthy matrix algebra, the following expression for the deputy satellite velocity vector is finally achieved.

$$
\begin{aligned}
\boldsymbol{v}_{d}^{O}= & {\left[\begin{array}{c}
V_{r J_{2}}+\delta V_{r J_{2}} \\
V_{t J_{2}}+\delta V_{t J_{2}} \\
V_{n J_{2}}+\delta V_{n J_{2}}
\end{array}\right]+V_{r J_{2}}\left[\begin{array}{c}
0 \\
\delta \theta+(c i) \delta \Omega \\
(s \theta) \delta i-(c \theta s i) \delta \Omega
\end{array}\right]+} \\
& V_{t J_{2}}\left[\begin{array}{c}
-\delta \theta-(c i) \delta \Omega \\
0 \\
(c \theta) \delta i+(s \theta s i) \delta \Omega
\end{array}\right]+V_{n J_{2}}\left[\begin{array}{c}
-(s \theta) \delta i+(c \theta s i) \delta \Omega \\
-(c \theta) \delta i-(s \theta s i) \delta \Omega \\
0
\end{array}\right]
\end{aligned}
$$

We divide the radial, tangential, and normal components of the chief satellite velocity vector into two parts. The first part is expressed in terms of the orbital elements and has the same form as that for unperturbed motion. The second part denoted by $\Delta$, 
is the variation caused solely by $J_{2}$ gravitational perturbation.

$$
\begin{array}{ll}
V_{r J_{2}}=V_{r}+\Delta V_{r} & \delta V_{r J_{2}}=\delta V_{r}+\delta \Delta V_{r} \\
V_{t J_{2}}=V_{t}+\Delta V_{t} & \delta V_{t J_{2}}=\delta V_{t}+\delta \Delta V_{t} \\
V_{n J_{2}}=V_{n}+\Delta V_{n} & \delta V_{n J_{2}}=\delta V_{n}+\delta \Delta V_{n}
\end{array}
$$

We next use equation (2.41) to write the first variation of $r$ as

$$
\delta r=\frac{r}{a} \delta a+\left[\frac{r^{2}}{p}\left(q_{1} s \theta-q_{2} c \theta\right)\right] \delta \theta-\left[\frac{r}{p}\left(2 a q_{1}+r c \theta\right)\right] \delta q_{1}-\left[\frac{r}{p}\left(2 a q_{2}+r s \theta\right)\right] \delta q_{2}
$$

Therefore, from equations (2.69) - (2.72), (2.83), (2.87), and (2.89), the overall relationship between $\boldsymbol{X}(t)$ and $\delta e(t)$ becomes

$$
\left[\begin{array}{l}
x \\
y \\
z
\end{array}\right]=\left[\begin{array}{c}
\frac{r}{a} \delta a+\left[\frac{r^{2}}{p}\left(q_{1} s \theta-q_{2} c \theta\right)\right] \delta \theta-\left[\frac{r}{p}\left(2 a q_{1}+r c \theta\right)\right] \delta q_{1}-\left[\frac{r}{p}\left(2 a q_{2}+r s \theta\right)\right] \delta q_{2} \\
r \delta \theta+r c i \delta \Omega \\
r s \theta \delta i-r c \theta s i \delta \Omega
\end{array}\right]
$$




$$
\begin{aligned}
{\left[\begin{array}{c}
\dot{x} \\
\dot{y} \\
\dot{z}
\end{array}\right]=} & {\left[\begin{array}{c}
\delta V_{r}+\delta \Delta V_{r} \\
\delta V_{t}+\delta \Delta V_{t} \\
\delta V_{n}+\delta \Delta V_{n}
\end{array}\right]+\left(V_{r}+\Delta V_{r}\right)\left[\begin{array}{c}
0 \\
\delta \theta+(c i) \delta \Omega \\
(s \theta) \delta i-(c \theta s i) \delta \Omega
\end{array}\right]+} \\
& \left(V_{t}+\Delta V_{t}\right)\left[\begin{array}{c}
-\delta \theta-(c i) \delta \Omega \\
0 \\
\varpi_{r} \\
z \\
-y
\end{array}\right]+\varpi_{t}\left[\begin{array}{c}
-(s \theta) \delta i+(c \theta s i) \delta \Omega \\
0 \\
-z \\
x
\end{array}\right]+\varpi_{n}\left[\begin{array}{c}
(s \theta) \delta i-(s \theta s i) \delta \Omega \\
-x \\
y \\
0
\end{array}\right]+
\end{aligned}
$$

We now use Lagrange's planetary equations to determine how the $J_{2}$ gravitational perturbation will affect the classical orbit element set $\boldsymbol{e}=\left(a, e, i, \Omega, \omega, M_{0}\right)^{T}$. The classical form of Lagrange's planetary equations is given by :[23]

$$
\begin{aligned}
\dot{a} & =\frac{2}{n a} \cdot \frac{\partial \mathscr{R}}{\partial M_{0}} \\
\dot{e} & =-\frac{\sqrt{1-e^{2}}}{n a^{2} e} \cdot \frac{\partial \mathscr{R}}{\partial \omega}+\frac{1-e^{2}}{n a^{2} e} \cdot \frac{\partial \mathscr{R}}{\partial M_{0}} \\
\dot{i} & =-\frac{1}{n a^{2} \sqrt{1-e^{2}} \sin i} \cdot \frac{\partial \mathscr{R}}{\partial \Omega}+\frac{\cot i}{n a^{2} \sqrt{1-e^{2}}} \cdot \frac{\partial \mathscr{R}}{\partial \omega} \\
\dot{\Omega} & =\frac{1}{n a^{2} \sqrt{1-e^{2}} \sin i} \cdot \frac{\partial \mathscr{R}}{\partial i} \\
\dot{\omega} & =-\frac{\cot i}{n a^{2} \sqrt{1-e^{2}}} \cdot \frac{\partial \mathscr{R}}{\partial i}+\frac{\sqrt{1-e^{2}}}{n a^{2} e} \cdot \frac{\partial \mathscr{R}}{\partial e} \\
\dot{M_{0}} & =-\frac{2}{n a} \cdot \frac{\partial \mathscr{R}}{\partial a}-\frac{1-e^{2}}{n a^{2} e} \cdot \frac{\partial \mathscr{R}}{\partial e}
\end{aligned}
$$


$\mathscr{R}$ is the disturbance potential function for the $J_{2}$ oblateness component of the gravitational spherical harmonics and it is expressed as

$$
\mathscr{R}=-\frac{\mu J_{2} R_{e}^{2}}{2 a^{3}\left(1-e^{2}\right)^{3}}(1+e \cos f)^{3}\left(3 \sin ^{2} i \sin ^{2} \theta-1\right)
$$

where $R_{e}$ is Earth equatorial radius. We must compute the partial derivatives of $\mathscr{R}$ with respect to the classical orbit elements. To develop expressions for $\partial \mathscr{R} / \partial e$, $\partial \mathscr{R} / \partial a$, and $\partial \mathscr{R} / \partial M_{0}$, we need to employ the following disturbed two-body motions identities.

$$
\begin{aligned}
\frac{\partial r}{\partial e} & =-a \cos f \\
\frac{\partial}{\partial e}\left(\frac{a}{r}\right) & =\left(\frac{a}{r}\right)^{2} \cos f \\
\frac{\partial f}{\partial e} & =\left(\frac{a}{r}+\frac{1}{1-e^{2}}\right) \sin f \\
\frac{\partial r}{\partial a} & =\frac{r}{a}-\frac{3 n e\left(t-t_{0}\right)}{2 \sqrt{1-e^{2}}} \sin f \\
\frac{\partial}{\partial a}\left(\frac{a}{r}\right) & =\frac{3 a n e\left(t-t_{0}\right)}{2 r^{2} \sqrt{1-e^{2}}} \sin f \\
\frac{\partial f}{\partial a} & =-\frac{3 a n\left(t-t_{0}\right) \sqrt{1-e^{2}}}{2 r^{2}} \\
\frac{\partial r}{\partial f} & =\frac{r^{2} e}{a\left(1-e^{2}\right)} \sin f \\
\frac{\partial f}{\partial M_{0}} & =\left(\frac{a}{r}\right)^{2} \sqrt{1-e^{2}}
\end{aligned}
$$

We prove these disturbed two-body motions identities one at a time in Appendix A. Computation of the partial derivatives of $\mathscr{R}$ with respect to the classical orbit elements reveals that there is an explicit linear function of time $t$ in the expression for $\partial \mathscr{R} / \partial a$. This explicit linear function of time creates two undesirable secular terms in equation (2.92f) and it will cause numerical complication when large values of $t$ are considered. To avoid any possible numerical errors associated with the time rate of change of the initial mean anomaly $M_{0}$, we proceed in the following manner. First 
we differentiate the mean anomaly relation $M=M_{0}+n\left(t-t_{0}\right)$ with respect to time $t$ and obtain

$$
\frac{d M}{d t}=\frac{d M_{0}}{d t}-\frac{3 n\left(t-t_{0}\right)}{2 a} \cdot \frac{d a}{d t}+n
$$

We next substitute expressions for $\dot{M}_{0}$ and $\dot{a}$ from equations (2.92f) and (2.92a) respectively into the above equation to get

$$
\dot{M}=-\frac{2}{n a}\left[\frac{\partial \mathscr{R}}{\partial a}+\frac{3 n\left(t-t_{0}\right)}{2 a} \cdot \frac{\partial \mathscr{R}}{\partial M_{0}}\right]-\frac{1-e^{2}}{n a^{2} e} \cdot \frac{\partial \mathscr{R}}{\partial e}+n
$$

The $\frac{3 n\left(t-t_{0}\right)}{2 a} \cdot \frac{\partial \mathscr{R}}{\partial M_{0}}$ term inside the above square brackets cancels two secular terms presented by $\partial \mathscr{R} / \partial a$. Thus the expression for the time rate of change of the mean anomaly $M$ does not contain an explicit linear function of time $t$ and we can use $\dot{M}$ instead of $\dot{M}_{0}$ in Lagrange's planetary equations to overcome any possible numerical difficulty. Noting that $\partial \mathscr{R} / \partial M=\partial \mathscr{R} / \partial M_{0}$, we can rewrite the Lagrange's planetary equations in the following form

$$
\begin{aligned}
\dot{a} & =\frac{2}{n a} \cdot \frac{\partial \mathscr{R}}{\partial M} \\
\dot{e} & =-\frac{\sqrt{1-e^{2}}}{n a^{2} e} \cdot \frac{\partial \mathscr{R}}{\partial \omega}+\frac{1-e^{2}}{n a^{2} e} \cdot \frac{\partial \mathscr{R}}{\partial M} \\
\dot{i} & =-\frac{1}{n a^{2} \sqrt{1-e^{2}} \sin i} \cdot \frac{\partial \mathscr{R}}{\partial \Omega}+\frac{\cot i}{n a^{2} \sqrt{1-e^{2}}} \cdot \frac{\partial \mathscr{R}}{\partial \omega} \\
\dot{\Omega} & =\frac{1}{n a^{2} \sqrt{1-e^{2}} \sin i} \cdot \frac{\partial \mathscr{R}}{\partial i} \\
\dot{\omega} & =-\frac{\cot i}{n a^{2} \sqrt{1-e^{2}}} \cdot \frac{\partial \mathscr{R}}{\partial i}+\frac{\sqrt{1-e^{2}}}{n a^{2} e} \cdot \frac{\partial \mathscr{R}}{\partial e} \\
\dot{M} & =n-\frac{3\left(t-t_{0}\right)}{a^{2}} \cdot \frac{\partial \mathscr{R}}{\partial M}-\frac{2}{n a} \cdot \frac{\partial \mathscr{R}}{\partial a}-\frac{1-e^{2}}{n a^{2} e} \cdot \frac{\partial \mathscr{R}}{\partial e}
\end{aligned}
$$


Subsequently the time variations of the orbit elements $(a, e, i, \Omega, \omega, M)$ due to $J_{2}$ gravitational perturbation become

$$
\begin{aligned}
& \dot{a}=\frac{\sigma \sqrt{\mu}\left(1+e c_{f}\right)^{4}}{a^{5 / 2} \eta^{9}}\left\{\left(3 s_{i}^{2} s_{\theta}^{2}-1\right) e s_{f}-\left(1+e c_{f}\right) s_{i}^{2} s_{2 \theta}\right\} \\
& \dot{e}=\frac{\sigma \sqrt{\mu}\left(1+e c_{f}\right)^{3}}{2 a^{7 / 2} \eta^{7}}\left\{\left(3 s_{i}^{2} s_{\theta}^{2}-1\right)\left(1+e c_{f}\right) s_{f}-\left(2 c_{f}+2 e-e s_{f}^{2}\right) s_{i}^{2} s_{2 \theta}\right\} \\
& \dot{i}=-\frac{\sigma \sqrt{\mu}\left(1+e c_{f}\right)^{3}}{4 a^{7 / 2} \eta^{7}}\left(s_{2 i} s_{2 \theta}\right) \\
& \dot{\omega}=-\frac{\sigma \sqrt{\mu}\left(1+e c_{f}\right)^{3}}{2 a^{7 / 2} \eta^{7}}\left\{\left(3 s_{i}^{2} s_{\theta}^{2}-1\right) c_{f}^{2}+s_{f} c_{f} s_{i}^{2} s_{2 \theta}-2 c_{i}^{2} s_{\theta}^{2}+\right. \\
& \dot{\Omega}=-\frac{\sigma \sqrt{\mu}\left(1+e c_{f}\right)^{3}}{a^{7 / 2} \eta^{7}}\left(c_{i} s_{\theta}^{2}\right) \\
& \left.\dot{M}=n+\frac{\sigma \sqrt{\mu}\left(1+e s_{f}\right)^{3}}{2 a^{7 / 2} \eta^{6}}\left\{\left(3 s_{i}^{2} s_{\theta}^{2}-1\right)\left(c_{f}^{2}-2\right)+s_{f} c_{f} s_{i}^{2} s_{2 \theta}+2 s_{i}^{2} s_{2 \theta}\right]\right\} \\
& \left.\frac{1}{e}\left[\left(3 s_{i}^{2} s_{\theta}^{2}-1\right) c_{f}+2 s_{f} s_{i}^{2} s_{2 \theta}\right]\right\}
\end{aligned}
$$

where $n=\sqrt{\mu / a^{3}}, \eta=\sqrt{1-e^{2}}$ and the shorthand notations $s_{\gamma}=\sin \gamma$ and $c_{\gamma}=\cos \gamma$ are used. We can further express the time rate of change of the true anomaly as

$\dot{f}=\frac{\sqrt{\mu}\left(1+e c_{f}\right)^{2}}{a^{3 / 2} \eta^{3}}+\frac{\sigma \sqrt{\mu}\left(1+e c_{f}\right)^{3}}{2 e a^{7 / 2} \eta^{7}}\left\{\left(3 s_{i}^{2} s_{\theta}^{2}-1\right)\left(1+e c_{f}\right) c_{f}+\left(2+e c_{f}\right) s_{f} s_{i}^{2} s_{2 \theta}\right\}$

For convenience, we assume all of the variables of the chief and the deputy satellites without overbar represent osculating elements. Recall that the instantaneous total angular velocity $\varpi$ for the osculating orbit elements of the chief satellite that includes the effect of $J_{2}$ gravitational perturbation was given by identities (2.67) and (2.68). We also note that the chief reference orbital plane can be defined by the position and velocity vectors' identities (2.69) and (2.70) respectively. Because the chief satellite 
velocity vector must be in the chief reference orbital plane, we should have $V_{n J_{2}}=0$. In other words

$$
\dot{i} \sin \theta=\dot{\Omega} \cos \theta \sin i
$$

Thus the instantaneous total angular velocity vector components of the chief satellite become

$$
\varpi_{r}=\frac{\dot{\Omega} \sin i}{\sin \theta} \quad \varpi_{t}=0 \quad \varpi_{n}=\dot{\Omega} \cos i+\dot{\theta}
$$

The chief total angular momentum that is always perpendicular to its instantaneous orbital plane is

$$
h=r^{2} \varpi_{n}=r^{2}(\dot{\Omega} \cos i+\dot{\theta})=\sqrt{\mu p}
$$

Employing the vis-viva integral and few perturbed two-body motions relationships [23], the chief satellite velocity vector components in terms of the chief osculating orbit elements are expressed by

$$
\begin{aligned}
V_{r J_{2}} & =\dot{r}=\sqrt{\frac{\mu}{p}}\left(q_{1} \sin \theta-q_{2} \cos \theta\right) \\
V_{t J_{2}} & =r \varpi_{n}=\sqrt{\frac{\mu}{p}}\left(1+q_{1} \cos \theta+q_{2} \sin \theta\right) \\
V_{n J_{2}} & =0
\end{aligned}
$$

Recall that equations (2.43) and (2.44) give the chief radial and tangential velocity components $V_{r}$ and $V_{t}$ for an unperturbed chief motion. We note that the above expressions for $V_{r J_{2}}$ and $V_{t J_{2}}$ are equal to $V_{r}$ and $V_{t}$ expressions in equations (2.43) 
and (2.44) respectively. Thus from equation (2.88) we can write

$$
\Delta V_{r}=\Delta V_{t}=\Delta V_{n}=0
$$

We also use identities in equation (2.103) to rewrite the equation (2.89) as follows

$$
\delta r=\frac{r}{a} \delta a+\frac{V_{r}}{V_{t}} r \delta \theta-\frac{r}{p}\left(2 a q_{1}+r \cos \theta\right) \delta q_{1}-\frac{r}{p}\left(2 a q_{2}+r \sin \theta\right) \delta q_{2}
$$

Finally substituting equations (2.101), (2.103), (2.104), and (2.105) into equations (2.90) and (2.91) yields the transformation matrix $\mathcal{T} \equiv[A(t)+\sigma B(t)]$ for osculating orbit elements. The refined form of this matrix is given in Appendix B. We note that the matrix $A(t)$ in the transformation matrix $\mathcal{T}$ has the same form as the matrix $A\left(\boldsymbol{e}_{c}\right)$ defined by equations (2.45) and (2.52) for an unperturbed elliptic chief orbit. ${ }^{[22]}$ However we must realize that these two matrices are not numerically the same because the $J_{2}$ perturbation is involved indirectly in the orbital elements of matrix $A(t)$ components. The inverse transformation matrix $\mathcal{T}^{-1}$ is given in Appendix $\mathrm{C}$.

\subsubsection{State Transition Matrix for the Relative Mean Orbit Elements $\Phi_{\delta \bar{e}}\left(t, t_{0}\right)$}

We now determine the state transition matrix $\Phi_{\delta \bar{e}}\left(t, t_{0}\right)$ for the relative mean orbit elements. We know that the mean orbit elements depict the long-term secular effects. The secular variations of the orbital elements caused by the $J_{2}$ gravitational 
perturbation after neglecting second order terms in $J_{2}$ are given by :[24]

$$
\begin{aligned}
\dot{\bar{a}} & =0 \\
\dot{\bar{e}} & =0 \\
\dot{\bar{i}} & =0 \\
\dot{\bar{\Omega}} & =-\frac{\sigma \sqrt{\mu}}{2 \bar{a}_{0}^{7 / 2} \bar{\eta}_{0}^{4}} \cos \bar{i}_{0} \\
\dot{\bar{\omega}} & =\frac{\sigma \sqrt{\mu}}{4 \bar{a}_{0}^{7 / 2} \bar{\eta}_{0}^{4}}\left(5 \cos ^{2} \bar{i}_{0}-1\right) \\
\dot{\bar{M}} & =\frac{\sqrt{\mu}}{\bar{a}_{0}^{3 / 2}}+\frac{\sigma \sqrt{\mu}}{4 \bar{a}_{0}^{7 / 2} \bar{\eta}_{0}^{3}}\left(3 \cos ^{2} \bar{i}_{0}-1\right)
\end{aligned}
$$

where $\bar{\eta}_{0}=\sqrt{1-\bar{q}_{10}^{2}-\bar{q}_{20}^{2}}$ and the subscript 0 indicates that the values are at time $t_{0}$. In this subsection, we represent the chief satellite mean orbit elements by $\overline{\boldsymbol{e}}=\left(\bar{a}, \bar{\theta}, \bar{i}, \bar{q}_{1}, \bar{q}_{2}, \bar{\Omega}\right)^{T}$. Considering only the secular variations produced by the $J_{2}$ gravitational perturbation, the chief satellite mean orbit elements can be expressed by

$$
\begin{aligned}
\bar{a} & =\bar{a}_{0} \\
\bar{e} & =\bar{e}_{0} \\
\bar{i} & =\bar{i}_{0} \\
\bar{q}_{1} & =\bar{e} \cos \overline{\bar{\omega}}=\bar{q}_{10} \cos \left[\dot{\bar{\omega}}\left(t-t_{0}\right)\right]-\bar{q}_{20} \sin \left[\dot{\bar{\omega}}\left(t-t_{0}\right)\right] \\
\bar{q}_{2} & =\bar{e} \sin \bar{\omega}=\bar{q}_{10} \sin \left[\dot{\bar{\omega}}\left(t-t_{0}\right)\right]+\bar{q}_{20} \cos \left[\dot{\bar{\omega}}\left(t-t_{0}\right)\right] \\
\bar{\Omega} & =\bar{\Omega}_{0}+\dot{\bar{\Omega}}\left(t-t_{0}\right)
\end{aligned}
$$


It is evident that the chief mean orbit elements are only functions of time and initial mean orbit elements. To obtain an expression for $\bar{\theta}$, we define

$$
\begin{aligned}
& \theta=f+\omega=\text { true argument of latitude } \\
& \lambda=M+\omega=\text { mean argument of latitude } \\
& F=E+\omega=\text { eccentric argument of latitude }
\end{aligned}
$$

From kepler's equation $M=E-e \sin E$, the relationship between $\lambda$ and $F$ becomes

$$
\begin{aligned}
\lambda & =(E+\omega)-e \sin E=F-\frac{e \sqrt{1-e^{2}} \sin (\theta-\omega)}{1+e \cos (\theta-\omega)} \\
& =F-\frac{\sqrt{1-q_{1}^{2}-q_{2}^{2}}\left(q_{1} \sin \theta-q_{2} \cos \theta\right)}{1+q_{1} \cos \theta+q_{2} \sin \theta}
\end{aligned}
$$

We use Figure 2.4 to attain a relationship between the eccentric argument of latitude $F$ and the true argument of latitude $\theta$. The point $P$ in the coordinate system $\{\varsigma, \varrho\}$

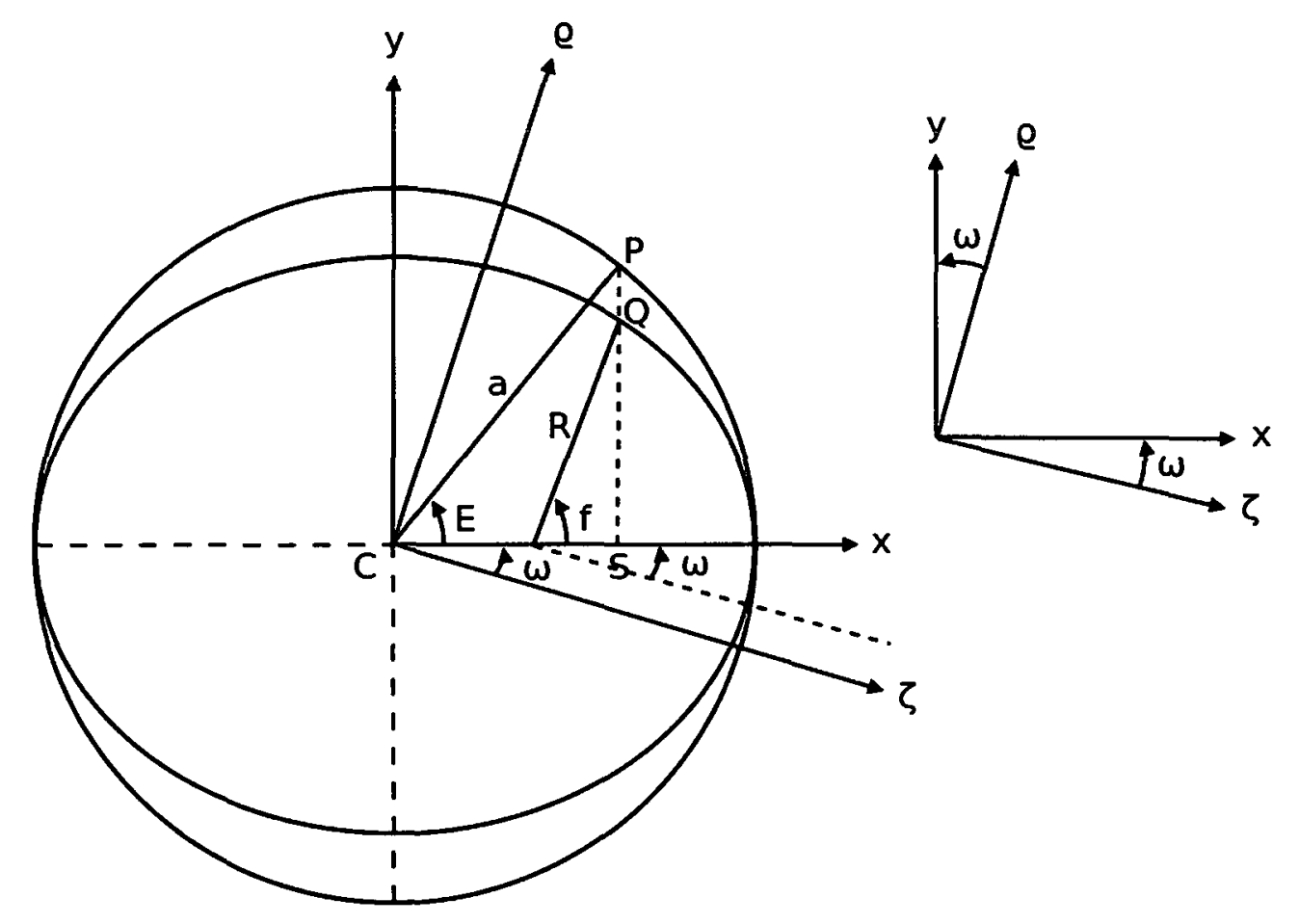

Figure 2.4: Illustration of the relationship between the eccentric argument of latitude $F$ and the true argument of latitude $\theta$ 
is specified by

$$
\varsigma=a \cos F \quad, \quad \varrho=a \sin F
$$

Also, the point $P$ in the coordinate system $\{x, y\}$ is defined by

$$
x=a e+r \cos f \quad, \quad y=\frac{r \sin f}{\sqrt{1-e^{2}}}
$$

From the relationship between two above coordinate systems, we write

$$
\begin{aligned}
& {\left[\begin{array}{l}
a \cos F \\
a \sin F
\end{array}\right]=\left[\begin{array}{cc}
\cos \omega & -\sin \omega \\
\sin \omega & \cos \omega
\end{array}\right]\left[\begin{array}{c}
a e+r \cos f \\
\frac{r \sin f}{\sqrt{1-e^{2}}}
\end{array}\right]} \\
& =\left[\begin{array}{l}
a e \cos \omega+r \cos \omega \cos f-\frac{r \sin \omega \sin f}{\sqrt{1-e^{2}}} \\
a e \sin \omega+r \sin \omega \cos f+\frac{r \cos \omega \sin f}{\sqrt{1-e^{2}}}
\end{array}\right]
\end{aligned}
$$

By defining

$$
\beta \equiv \frac{1}{\left(1-e^{2}\right)+\sqrt{1-e^{2}}}=\frac{1}{\left(1-q_{1}^{2}-q_{2}^{2}\right)+\sqrt{1-q_{1}^{2}-q_{2}^{2}}}
$$

the eccentric argument of latitude $F$ is expressed in terms of the orbit elements $\boldsymbol{e}=\left(a, \theta, i, q_{1}, q_{2}, \Omega\right)^{T}$ by

$$
\tan F=\frac{r\left(1+\beta q_{1}^{2}\right) \sin \theta-\beta r q_{1} q_{2} \cos \theta+a q_{2}}{r\left(1+\beta q_{2}^{2}\right) \cos \theta-\beta r q_{1} q_{2} \sin \theta+a q_{1}}
$$

where

$$
r=\frac{a\left(1-q_{1}^{2}-q_{2}^{2}\right)}{1+q_{1} \cos \theta+q_{2} \sin \theta}
$$


We now employ equations (2.109) and (2.114) to write the relationship between $\lambda$ and $\theta$ as

$\lambda=\tan ^{-1}\left(\frac{r\left(1+\beta q_{1}^{2}\right) \sin \theta-\beta r q_{1} q_{2} \cos \theta+a q_{2}}{r\left(1+\beta q_{2}^{2}\right) \cos \theta-\beta r q_{1} q_{2} \sin \theta+a q_{1}}\right)-\frac{\sqrt{1-q_{1}^{2}-q_{2}^{2}}\left(q_{1} \sin \theta-q_{2} \cos \theta\right)}{1+q_{1} \cos \theta+q_{2} \sin \theta}$

We next find an expression for the mean orbit element $\bar{\lambda}$. We define a function $G$ and use the secular variations in equation (2.106) to write

$$
G \equiv \bar{\lambda}-\bar{\lambda}_{0}=\left(\bar{M}-\bar{M}_{0}\right)+\left(\bar{\omega}-\bar{\omega}_{0}\right)=\dot{\bar{M}}\left(t-t_{0}\right)+\dot{\bar{\omega}}\left(t-t_{0}\right)
$$

The mean orbit element $\bar{\lambda}$ becomes

$$
\bar{\lambda}=\bar{\lambda}_{0}+(\dot{\bar{M}}+\dot{\bar{\omega}})\left(t-t_{0}\right)
$$

Substituting $\bar{\lambda}$ from equation (2.118) and other mean orbit elements from equation (2.107) into equation (2.116) yields a relation for the mean orbit element $\bar{\theta}$. The state transition matrix for the relative mean orbit elements $\Phi_{\delta \bar{e}}\left(t, t_{0}\right)$ is obtained by taking first-order Taylor-series expansions of equations (2.107) and (2.117) about the chief satellite mean motion. This matrix $\Phi_{\delta \bar{e}}\left(t, t_{0}\right)$ is given in Appendix D as a function of time and the chief satellite mean orbit elements.

\subsubsection{Transformation Matrix Between the Relative Mean and the Relative Osculating Orbit Elements $D(t)$}

The osculating orbit elements can be expressed as functions of the mean orbit elements by

$$
e_{\mathrm{osc}}=e_{\mathrm{mean}}+\Delta e_{\mathrm{p}}
$$


where

$$
\Delta \boldsymbol{e}_{\mathrm{p}}=-J_{2} R_{e}^{2} \mathscr{F}\left(\boldsymbol{e}_{\text {mean }}, W_{1}\right)
$$

$\Delta e_{\mathrm{p}}$ designates the short-period and the long-period oscillations caused in the osculating orbit elements by the $J_{2}$ gravitational perturbation. The short- and longperiod oscillations are in fact periodic deviation from the mean orbit elements. Recall that the mean orbit elements only represent the long-term secular drift in the osculating orbit elements. The transformation function $\mathscr{F}\left(\boldsymbol{e}_{\text {mean }}, W_{1}\right)$ depends on a generating function $W_{1}$ which is defined by :[17]

$$
W_{1}=W_{1}^{(l p)}+W_{1}^{(s p 1)}+W_{1}^{(s p 2)}
$$

Note that the short-period oscillation has been devided into two parts. Employing the normalized Delaunay variables $(l, g, h, L, G, H)^{T}$ as orbit elements and the true anomaly $f$, the generating functions $W_{1}^{(l p)}, W_{1}^{(s p 1)}$, and $W_{1}^{(s p 2)}$ are given by

$$
\begin{aligned}
W_{1}^{(l p)} & =-\left(\frac{1}{32 G^{3}}\right) \cdot\left(1-\frac{G^{2}}{L^{2}}\right) \cdot\left(1-5 \frac{H^{2}}{G^{2}}\right)^{-1} \cdot\left(1-16 \frac{H^{2}}{G^{2}}+15 \frac{H^{4}}{G^{4}}\right) \sin (2 g) \\
W_{1}^{(s p 1)} & =\left(\frac{1}{4 G^{3}}\right) \cdot\left(-1+\frac{3 H^{2}}{G^{2}}\right) \cdot(f-1+e \sin f) \\
W_{1}^{(s p 2)} & =\frac{3}{8 G^{3}}\left(1-\frac{H^{2}}{G^{2}}\right) \cdot\left[\sin (2 f+2 g)+e \sin (f+2 g)+\frac{e}{3} \sin (3 f+2 g)\right]
\end{aligned}
$$


The Delaunay variables are defined as

$$
\begin{aligned}
l & \equiv \text { mean anomaly } M \\
g & \equiv \text { argument of perigee } \omega \\
h & \equiv \text { longitude of ascending node } \Omega \\
L & \equiv \sqrt{\mu a} \\
G & \equiv L \sqrt{1-e^{2}} \\
H & \equiv G \cos i
\end{aligned}
$$

Using the generating function $W_{1}$ and the transformation function $\mathscr{F}, \Delta e_{\mathrm{p}}$ is attained and the osculating orbit elements $\boldsymbol{e}_{\mathrm{osc}}$ is expressed in terms of the mean orbit elements $e_{\text {mean }}$ by

$$
e_{\mathrm{osc}}=e_{\text {mean }}-J_{2} R_{e}^{2}\left[e^{(l p)}+e^{(s p 1)}+e^{(s p 2)}\right]
$$

Finally, the transformation matrix $D(t)$ for the relative orbit elements is acquired by taking the partial derivatives of the osculating orbit elements with respect to the mean orbit elements

$$
D(t)=\frac{\partial e_{\mathrm{osc}}}{\partial e_{\mathrm{mean}}}=I-J_{2} R_{e}^{2}\left[D^{(l p)}(t)+D^{(s p 1)}(t)+D^{(s p 2)}(t)\right]
$$

The column vectors $\boldsymbol{e}^{(l p)}, \boldsymbol{e}^{(s p 1)}, \boldsymbol{e}^{(s p 2)}$ and matrices $D^{(l p)}, D^{(s p 1)}$, and $D^{(s p 2)}$ are given in Appendices $\mathrm{E}$ and $\mathrm{F}$ respectively in terms of the chief satellite mean orbit elements $e_{\text {mean }}$ 


\subsection{Numerical Evaluation}

To evaluate the accuracy of Gim-Alfriend closed-form solution to the relative motion of satellites, we execute the following sequence of steps in our numerical simulation for each pair of the formation flying chief and deputy satellites.

1. We numerically integrate the equations of motion of both chief and deputy satellites in the Earth-centered-inertial (ECI) reference frame with $J_{2}$ to $J_{6}$ gravitational perturbations included.

2. Utilizing the chief and deputy satellites position and velocity vectors in the ECI frame, we compute directly the deputy satellite actual relative position and velocity vectors in the chief LVLH curvilinear frame.

3. We compute the predicted relative motion of the deputy satellite in the chief LVLH curvilinear frame via Gim-Alfriend state transition matrix.

4. We compare the actual relative position and velocity vectors from step 2 with the predicted relative position and velocity vectors from step 3

The equations of motion for chief and deputy satellites in step 1 are expressed by Newton's second law as

$$
\ddot{\boldsymbol{r}}=-\frac{\mu}{r^{3}} \boldsymbol{r}+\boldsymbol{a}_{p}
$$

which, for numerical integration, we reduce them to the following set of first-order differential equations

$$
\left\{\begin{array}{l}
\dot{\boldsymbol{r}}=\boldsymbol{r} \\
\dot{\boldsymbol{v}}=-\frac{\mu}{r^{3}} \boldsymbol{r}+\boldsymbol{a}_{p}
\end{array}\right.
$$


In the above equations $\boldsymbol{r}$ and $\boldsymbol{v}$ are the position and velocity vectors of the satellite in the ECI reference frame, $\boldsymbol{a}_{p}$ is the vector sum of all the perturbing accelerations and $\mu$ is the Earth's gravitational constant. We know that one of the most significant perturbing accelerations for low-Earth orbits is due to a non-spherical Earth. We assume a spheroid of revolution about a spin axis can closely represent the shape and mass distribution of the Earth in our simulation and therefore we only consider those perturbations that are caused by zonal harmonics $J_{2}$ to $J_{6}$ of Earth gravitation potential. We also understand that the perturbation due to atmospheric drag would need to be considered for orbits with perigee altitude less than $1000 \mathrm{~km}$, but we further assume that all the formation flying spacecrafts in our simulation are of equal type and build, and subsequently they have the same ballistic coefficient. This means that the differential drag effect on the close relative motion is negligible for a time period of at least several orbits and we can safely ignore this factor in our numerical evaluation.

The perturbing acceleration vector $\boldsymbol{a}_{p}$ in the ECI reference frame can be determined from the gravity potential function $U(\boldsymbol{r})$ as follows

$$
\boldsymbol{a}_{p}=\nabla U(\boldsymbol{r})
$$

The gravity potential $U(\boldsymbol{r})$ takes on its most famous form below :[25]

$$
U(r, \phi)=-\frac{\mu}{r}\left[1-\sum_{k=2}^{\infty}\left(\frac{R_{e}}{r}\right)^{k} J_{k} P_{k}(\sin \phi)\right]
$$

where $r$ and $\phi$ are the spherical coordinates of the Earth mass element and $P_{k}(\sin \phi)$ is the $k^{\text {th }}$ order Legendre polynomial of $\sin \phi$. For the Earth, the first six zonal 
harmonics are given by :[26]

$$
\begin{aligned}
& J_{2}=2.08262668355 \times 10^{-3} \\
& J_{3}=-2.53265648533 \times 10^{-6} \\
& J_{4}=-1.61962159137 \times 10^{-6} \\
& J_{5}=-2.27296082869 \times 10^{-7} \\
& J_{6}=5.40681239107 \times 10^{-7}
\end{aligned}
$$

The gravity potential function $U(r, \phi)$ for the $J_{2}$ through $J_{6}$ gravitational perturbations is expressed by :[23]

$$
\begin{aligned}
U(r, \phi)= & -\frac{J_{2}}{2} \frac{\mu}{r}\left(\frac{R_{e}}{r}\right)^{2}\left(3 \sin ^{2} \phi-1\right) \\
& -\frac{J_{3}}{2} \frac{\mu}{r}\left(\frac{R_{e}}{r}\right)^{3}\left(5 \sin ^{3} \phi-3 \sin \phi\right) \\
& -\frac{J_{4}}{8} \frac{\mu}{r}\left(\frac{R_{e}}{r}\right)^{4}\left(35 \sin ^{4} \phi-30 \sin ^{2} \phi+3\right) \\
& -\frac{J_{5}}{8} \frac{\mu}{r}\left(\frac{R_{e}}{r}\right)^{5}\left(63 \sin ^{5} \phi-70 \sin ^{3} \phi+15 \sin \phi\right) \\
& -\frac{J_{6}}{16} \frac{\mu}{r}\left(\frac{R_{e}}{r}\right)^{6}\left(231 \sin ^{6} \phi-315 \sin ^{4} \phi+105 \sin ^{2} \phi-5\right)
\end{aligned}
$$

Computing the gradient of $U(r, \phi)$ and making use of $\sin \phi=Z / r$, the perturbing accelerations $\boldsymbol{a}_{J_{i}}$ due to $J_{i}$ harmonics in terms of the ECI coordinates are obtained by :[23]

$$
\boldsymbol{a}_{J_{2}}=-\frac{3}{2} J_{2}\left(\frac{\mu}{r^{2}}\right)\left(\frac{R_{e}}{r}\right)^{2}\left[\begin{array}{l}
\left(1-5\left(\frac{Z}{r}\right)^{2}\right) \frac{X}{r} \\
\left(1-5\left(\frac{Z}{r}\right)^{2}\right) \frac{Y}{r} \\
\left(3-5\left(\frac{Z}{r}\right)^{2}\right) \frac{Z}{r}
\end{array}\right]
$$




$$
\begin{aligned}
& \boldsymbol{a}_{J_{3}}=\frac{1}{2} J_{3}\left(\frac{\mu}{r^{2}}\right)\left(\frac{R_{e}}{r}\right)^{3}\left[\begin{array}{c}
5\left(7\left(\frac{Z}{r}\right)^{3}-3\left(\frac{Z}{r}\right)\right) \frac{X}{r} \\
5\left(7\left(\frac{Z}{r}\right)^{3}-3\left(\frac{Z}{r}\right)\right) \frac{Y}{r} \\
3\left(1-10\left(\frac{Z}{r}\right)^{2}+\frac{35}{3}\left(\frac{Z}{r}\right)^{4}\right)
\end{array}\right] \\
& \boldsymbol{a}_{J_{4}}=\frac{5}{8} J_{4}\left(\frac{\mu}{r^{2}}\right)\left(\frac{R_{e}}{r}\right)^{4}\left[\begin{array}{l}
\left(3-42\left(\frac{Z}{r}\right)^{2}+63\left(\frac{Z}{r}\right)^{4}\right) \frac{X}{r} \\
\left(3-42\left(\frac{Z}{r}\right)^{2}+63\left(\frac{Z}{r}\right)^{4}\right) \frac{Y}{r} \\
\left(15-70\left(\frac{Z}{r}\right)^{2}+63\left(\frac{Z}{r}\right)^{4}\right) \frac{Z}{r}
\end{array}\right] \\
& \boldsymbol{a}_{J_{5}}=\frac{1}{8} J_{5}\left(\frac{\mu}{r^{2}}\right)\left(\frac{R_{e}}{r}\right)^{5}\left[\begin{array}{c}
3\left(35\left(\frac{Z}{r}\right)-210\left(\frac{Z}{r}\right)^{3}+231\left(\frac{Z}{r}\right)^{5}\right) \frac{X}{r} \\
3\left(35\left(\frac{Z}{r}\right)-210\left(\frac{Z}{r}\right)^{3}+231\left(\frac{Z}{r}\right)^{5}\right) \frac{Y}{r} \\
\left(693\left(\frac{Z}{r}\right)^{6}-945\left(\frac{Z}{r}\right)^{4}+315\left(\frac{Z}{r}\right)^{2}-15\right)
\end{array}\right] \\
& \boldsymbol{a}_{J_{6}}=-\frac{1}{16} J_{6}\left(\frac{\mu}{r^{2}}\right)\left(\frac{R_{e}}{r}\right)^{6}\left[\begin{array}{c}
\left(35-945\left(\frac{Z}{r}\right)^{2}+3465\left(\frac{Z}{r}\right)^{4}-3003\left(\frac{Z}{r}\right)^{6}\right) \frac{X}{r} \\
\left(35-945\left(\frac{Z}{r}\right)^{2}+3465\left(\frac{Z}{r}\right)^{4}-3003\left(\frac{Z}{r}\right)^{6}\right) \frac{Y}{r} \\
\left(245-2205\left(\frac{Z}{r}\right)^{2}+4851\left(\frac{Z}{r}\right)^{4}-3003\left(\frac{Z}{r}\right)^{6}\right) \frac{Z}{r}
\end{array}\right]
\end{aligned}
$$

where $X, Y$, and $Z$ are the satellite position vector coordinates in the ECI reference frame. Note that $\boldsymbol{a}_{p}$ is the vector sum of all the above perturbing accelerations.

There is an alternative method that we can employ to determine the perturbing acceleration vector $\boldsymbol{a}_{p}$ in the ECI reference frame. Because we can express the gravity potential function $U(\boldsymbol{r})$ in spherical coordinates as $U(\boldsymbol{r})=U(r, \theta, i)$, with $\theta$ and $i$ being argument of latitude and inclination respectively, then the perturbing 
acceleration $\boldsymbol{a}_{p}$ components are given in the LVLH rectilinear frame by :[27]

$$
\begin{aligned}
a_{r}= & \frac{\partial U}{\partial r}=\frac{\mu}{r^{2}}\left\{\frac{3}{2} J_{2}\left(\frac{R_{e}}{r}\right)^{2}\left(3 A^{2}-1\right)+2 J_{3}\left(\frac{R_{e}}{r}\right)^{3}\left(5 A^{3}-3 A\right)\right. \\
& +\frac{3}{8} J_{4}\left(\frac{R_{e}}{r}\right)^{4}\left(35 A^{4}-30 A^{2}+3\right)+\frac{3}{4} J_{5}\left(\frac{R_{e}}{r}\right)^{5}\left(63 A^{5}-70 A^{3}+15 A\right) \\
& \left.+\frac{7}{16} J_{6}\left(\frac{R_{e}}{r}\right)^{6}\left(231 A^{6}-315 A^{4}+105 A^{2}-5\right)\right\} \\
a_{\theta}= & \frac{1}{r} \frac{\partial U}{\partial \theta}=-\frac{\mu}{r^{2}} \sin i \cos \theta\left\{3 J_{2}\left(\frac{R_{e}}{r}\right)^{2} A+\frac{3}{2} J_{3}\left(\frac{R_{e}}{r}\right)^{3}\left(5 A^{2}-1\right)\right. \\
& +\frac{5}{2} J_{4}\left(\frac{R_{e}}{r}\right)^{4}\left(7 A^{3}-3 A\right)+\frac{15}{8} J_{5}\left(\frac{R_{e}}{r}\right)^{5}\left(21 A^{4}-14 A^{2}+1\right) \\
& \left.+\frac{21}{8} J_{6}\left(\frac{R_{e}}{r}\right)^{6}\left(33 A^{5}-30 A^{3}+5 A\right)\right\} \\
a_{h}= & \frac{1}{r \sin \theta} \frac{\partial U}{\partial i}=-\frac{\mu}{r^{2}} \cos i\left\{3 J_{2}\left(\frac{R_{e}}{r}\right)^{2} A+\frac{3}{2} J_{3}\left(\frac{R_{e}}{r}\right)^{3}\left(5 A^{2}-1\right)\right. \\
& +\frac{5}{2} J_{4}\left(\frac{R_{e}}{r}\right)^{4}\left(7 A^{3}-3 A\right)+\frac{15}{8} J_{5}\left(\frac{R_{e}}{r}\right)^{5}\left(21 A^{4}-14 A^{2}+1\right) \\
& \left.+\frac{21}{8} J_{6}\left(\frac{R_{e}}{r}\right)^{6}\left(33 A^{5}-30 A^{3}+5 A\right)\right\}
\end{aligned}
$$

where $A=\sin i \sin \theta$. Subsequently a simple application of the direction cosine matrix $[\mathcal{C N}]^{T}$ from identity $(2.34 \mathrm{a})$ yields the perturbing acceleration vector $\boldsymbol{a}_{p}$ in the ECI reference frame.

Having expressed the perturbing acceleration $\boldsymbol{a}_{p}$ components in the LVLH rectilinear coordinate frame, we can utilize the Gaussian form of the Lagrange planetary equations to write the time rates of change of the classical orbital elements 
$(a, e, i, \Omega, \omega, M)^{T}$ as follows

$$
\begin{aligned}
\dot{a} & =\frac{2}{n \sqrt{1-e^{2}}}\left[e \sin f \cdot a_{r}+\frac{p}{r} \cdot a_{\theta}\right] \\
\dot{e} & =\frac{\sqrt{1-e^{2}}}{n a}\left[\sin f \cdot a_{r}+\left(\cos f+\frac{e+\cos f}{1+e \cos f}\right) \cdot a_{\theta}\right] \\
\dot{i} & =\frac{r \cos \theta}{n a^{2} \sqrt{1-e^{2}}} \cdot a_{h} \\
\dot{\Omega} & =\frac{r \sin \theta}{n a^{2} \sqrt{1-e^{2}} \sin i} \cdot a_{h} \\
\dot{\omega} & =\frac{\sqrt{1-e^{2}}}{n a e}\left[-\cos f \cdot a_{r}+\sin f\left(1+\frac{r}{p}\right) \cdot a_{\theta}\right]-\frac{r \cot i \sin \theta}{\sqrt{\mu p}} \cdot a_{h} \\
\dot{M} & =n+\frac{1}{n a^{2} e}\left[(p \cos f-2 e r) \cdot a_{r}-(p+r) \sin f \cdot a_{\theta}\right]
\end{aligned}
$$

The above set of equations allows us to propagate directly the classical orbital elements of the chief and deputy satellites forward in time and then convert them into position and velocity vectors in the ECI frame. This effectively alleviates the need to implement equation (2.127) and equations (2.132) to (2.136) in our simulation codes. However I implemented and utilized all the above redundant methods in my simulations to detect any computational failures in step 2 .

In step 2, we utilize the chief and deputy satellites osculating coordinates in the ECI frame and a set of mathematical formulations mainly developed by professor Penina Axelrad ${ }^{[11]}$ from university of Colorado, to directly compute the deputy satellite actual relative position and velocity vectors in the chief LVLH curvilinear frame. Prof. Axelrad formulations are explained as follows. We first represent the curvilinear LVLH frame axes at the chief satellite by the radial $\hat{\boldsymbol{e}}_{\boldsymbol{x}}$, in-track $\hat{\boldsymbol{e}}_{y}$, and cross-track $\hat{\boldsymbol{e}}_{z}$ unit vectors. We further use the unit vector triad $\left\{\hat{\boldsymbol{o}}_{r}, \hat{\boldsymbol{o}}_{\theta}, \hat{\boldsymbol{o}}_{h}\right\}$ to describe the orientation of the rectilinear LVLH coordinate frame at the chief satellite. The unit vector $\hat{\boldsymbol{o}}_{r}$ is in the chief orbit radius direction, the unit vector $\hat{\boldsymbol{o}}_{h}$ is parallel to the chief orbit angular momentum vector and the unit vector $\hat{\boldsymbol{o}}_{\theta}$ completes the right-hand 
coordinates system. These unit vectors are obtained by

$$
\begin{aligned}
\hat{\boldsymbol{o}}_{r} & =\frac{\boldsymbol{r}}{r} \\
\hat{\boldsymbol{o}}_{\theta} & =\hat{\boldsymbol{o}}_{h} \times \hat{\boldsymbol{o}}_{r} \\
\hat{\boldsymbol{o}}_{h} & =\frac{\boldsymbol{r} \times \boldsymbol{v}}{|\boldsymbol{r} \times \boldsymbol{v}|}
\end{aligned}
$$

where $\boldsymbol{r}$ and $\boldsymbol{v}$ are the osculating position and velocity vectors of the chief satellite in the ECI frame. The time derivatives of the unit vectors $\hat{\boldsymbol{o}}_{r}, \hat{\boldsymbol{o}}_{\theta}$, and $\hat{\boldsymbol{o}}_{h}$ are also obtained by

$$
\begin{aligned}
& \dot{\hat{\boldsymbol{o}}}_{r}=\frac{\boldsymbol{v}}{r}-\frac{\boldsymbol{r} \cdot \boldsymbol{v}}{r^{3}} \boldsymbol{r} \\
& \dot{\hat{\boldsymbol{o}}}_{\theta}=\dot{\hat{\boldsymbol{o}}}_{h} \times \hat{\boldsymbol{o}}_{r}+\hat{\boldsymbol{o}}_{h} \times \dot{\hat{\boldsymbol{o}}}_{r} \\
& \dot{\hat{\boldsymbol{o}}}_{h}=\frac{\boldsymbol{r} \times \boldsymbol{a}}{|\boldsymbol{r} \times \boldsymbol{v}|}-\frac{(\boldsymbol{r} \times \boldsymbol{v}) \cdot(\boldsymbol{r} \times \boldsymbol{a})}{|\boldsymbol{r} \times \boldsymbol{v}|^{3}}(\boldsymbol{r} \times \boldsymbol{v})
\end{aligned}
$$

where $\boldsymbol{a}$ is the chief satellite acceleration vector in the ECI frame. It is clear that the unit vectors $\hat{\boldsymbol{o}}_{r}, \hat{\boldsymbol{o}}_{\theta}$, and $\hat{\boldsymbol{o}}_{h}$ coincide with unit vectors $\hat{\boldsymbol{e}}_{x}, \hat{\boldsymbol{e}}_{y}$, and $\hat{\boldsymbol{e}}_{z}$ respectively at the chief. We now define a reference sphere of radius $r$ tangent to the local horizon $\left\{\hat{\boldsymbol{o}}_{\theta}, \hat{\boldsymbol{o}}_{h}\right\}$ plane at the chief. Thus in the chief satellite LVLH curvilinear frame, the radial component of the relative position $x$ is the difference between $r_{d}$ and $r$, and the curvilinear in-track and cross-track relative positions, $y$ and $z$, are measured along the surface of the chief reference sphere as indicated in Figure 2.5. 


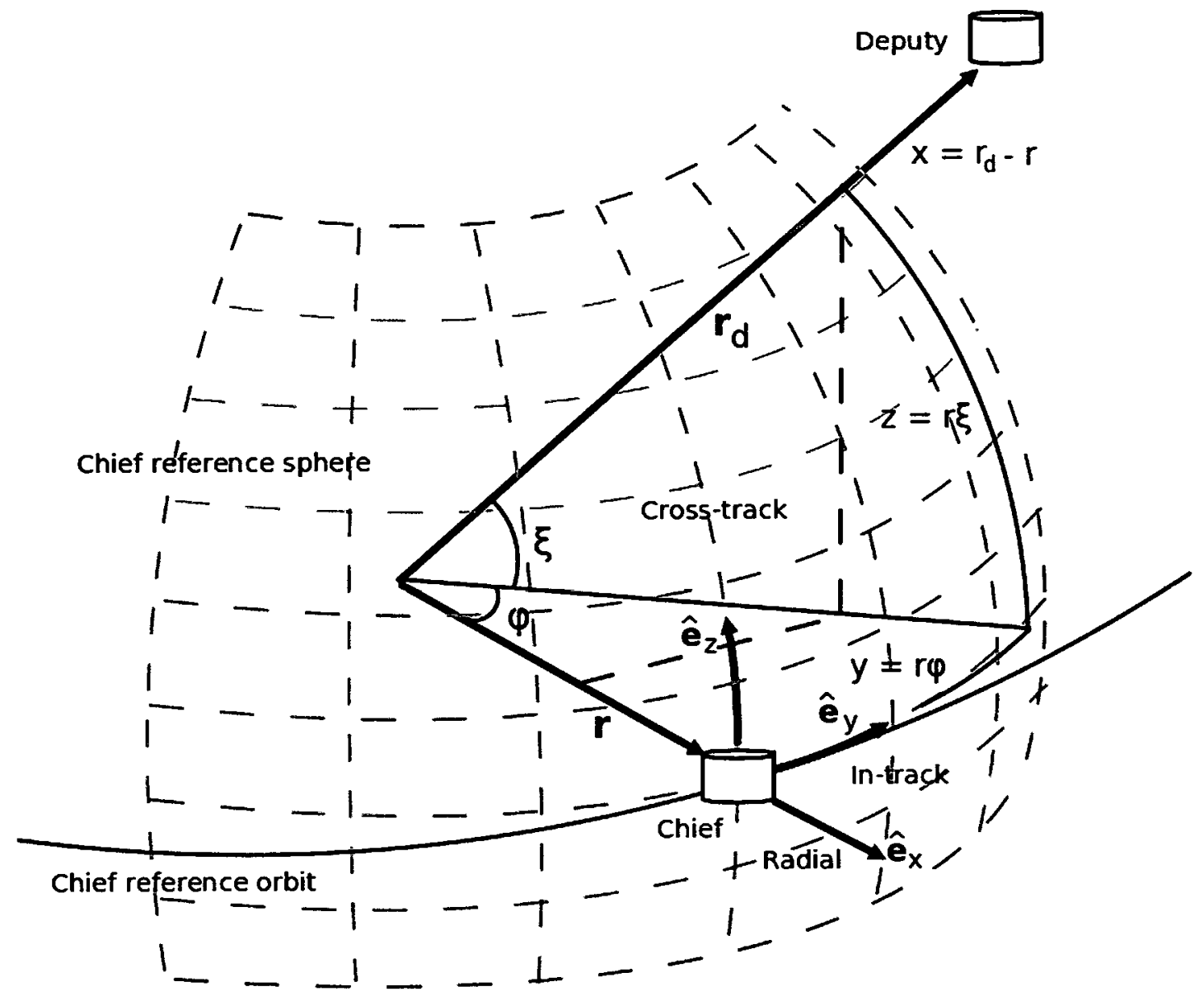

Figure 2.5: Illustration of the deputy satellite relative coordinates in the chief LVLH curvilinear frame

Mathematically the deputy satellite relative coordinates in the chief LVLH curvilinear frame are expressed as

$$
\begin{aligned}
& x=r_{d}-r \\
& \dot{x}=\frac{\boldsymbol{r}_{d} \cdot \boldsymbol{v}_{d}}{r_{d}}-\frac{\boldsymbol{r} \cdot \boldsymbol{v}}{r} \\
& y=r \phi \\
& \dot{y}=\frac{\boldsymbol{r} \cdot \boldsymbol{v}}{r} \phi+r \dot{\phi} \\
& z=r \xi \\
& \dot{z}=\frac{\boldsymbol{r} \cdot \boldsymbol{v}}{r} \xi+r \dot{\xi}
\end{aligned}
$$


where $\boldsymbol{r}_{d}$ and $\boldsymbol{v}_{d}$ are the deputy satellite osculating position and velocity vectors in the ECI frame, and $\phi$ and $\xi$ are the in-track and cross-track angular distances of the deputy satellite in the chief LVLH curvilinear frame. The $\phi$ and $\xi$ angles and their time derivatives are given by

$$
\begin{aligned}
& \phi=\tan ^{-1}\left(\frac{\hat{\boldsymbol{o}}_{r, d} \cdot \hat{\boldsymbol{o}}_{\theta}}{\hat{\boldsymbol{o}}_{r, d} \cdot \hat{\boldsymbol{o}}_{r}}\right) \\
& \dot{\phi}=\frac{1}{1+\tan ^{2}(\phi)}\left[\frac{1}{\hat{\boldsymbol{o}}_{r, d} \cdot \hat{\boldsymbol{o}}_{r}}\left(\hat{\boldsymbol{o}}_{r, d} \cdot \dot{\hat{\boldsymbol{o}}}_{\theta}+\dot{\hat{\boldsymbol{o}}}_{r, d} \cdot \hat{\boldsymbol{o}}_{\theta}\right)-\right. \\
& \left.\frac{\hat{\boldsymbol{o}}_{r, d} \cdot \hat{\boldsymbol{o}}_{\theta}}{\left(\hat{\boldsymbol{o}}_{r, d} \cdot \hat{\boldsymbol{o}}_{r}\right)^{2}}\left(\hat{\boldsymbol{o}}_{r, d} \cdot \dot{\hat{\boldsymbol{o}}}_{r}+\dot{\hat{\boldsymbol{o}}}_{r, d} \cdot \hat{\boldsymbol{o}}_{r}\right)\right] \\
& \xi=\sin ^{-1}\left(\hat{\boldsymbol{o}}_{r, d} \cdot \hat{\boldsymbol{o}}_{h}\right) \\
& \dot{\xi}=\frac{1}{\cos (\xi)}\left[\hat{\boldsymbol{o}}_{r, d} \cdot \dot{\hat{\boldsymbol{o}}}_{h}+\dot{\hat{\boldsymbol{o}}}_{r, d} \cdot \hat{\boldsymbol{o}}_{h}\right]
\end{aligned}
$$

The $\hat{\boldsymbol{o}}_{r, d}$ is the fundamental radial unit vector of the deputy rectilinear LVLH frame and $\dot{\hat{\boldsymbol{o}}}_{r, d}$ is its time derivative. They are obtained by

$$
\begin{aligned}
& \hat{\boldsymbol{o}}_{r, d}=\frac{\boldsymbol{r}_{d}}{r_{d}} \\
& \dot{\hat{\boldsymbol{o}}}_{r, d}=\frac{\boldsymbol{v}_{d}}{r_{d}}-\frac{\boldsymbol{r}_{d} \cdot \boldsymbol{v}_{d}}{r_{d}^{3}} \boldsymbol{r}_{d}
\end{aligned}
$$

In step 3 , we utilize equation (2.55) to predict the relative instantaneous state vector $\boldsymbol{X}=(x, \dot{x}, y, \dot{y}, z, \dot{z})^{T}$ of the deputy satellite in the chief LVLH curvilinear frame at any given time $t$. Using equation (2.61), we execute the following tasks to compute the Gim-Alfriend state transition matrix $\Phi_{\boldsymbol{X}}\left(t, t_{0}\right)$

- We evaluate the transformation matrix $[A(t)+\sigma B(t)]$ at times $t_{0}$ and $t$ by employing the chief satellite osculating orbit elements $\boldsymbol{e}=\left(a, \theta, i, q_{1}, q_{2}, \Omega\right)^{T}$.

- We evaluate the state transition matrix for the relative mean orbit elements $\Phi_{\delta \bar{e}}\left(t, t_{0}\right)$ and the transformation matrix between the relative mean and the relative osculating orbit elements $D(t)$, at times $t_{0}$ and $t$ by employing the chief 
satellite mean orbit elements $\overline{\boldsymbol{e}}=\left(\bar{a}, \bar{\theta}, \bar{i}, \bar{q}_{1}, \bar{q}_{2}, \bar{\Omega}\right)^{T}$.

Recall that the chief satellite osculating orbit elements $\boldsymbol{e}$ is easily obtained from the corresponding classical orbit elements $(a, e, i, \Omega, \omega, M)^{T}$ by using simple identities $\theta=f+\omega, q_{1}=e \cos \omega$, and $q_{2}=e \sin \omega$. The chief satellite classical orbit elements have already been determined for any time $t$ in step 1 . In an analogous manner, the chief satellite mean orbit elements $\bar{e}$ at any time $t$ can be obtained from the corresponding mean classical orbit elements $(\bar{a}, \bar{e}, \bar{i}, \bar{\Omega}, \bar{\omega}, \bar{M})^{T}$. The chief satellite mean classical orbit elements at any given time $t$ are obtained by

$$
\begin{aligned}
& \bar{a}=\bar{a}_{0}+\dot{\bar{a}}\left(t-t_{0}\right) \\
& \bar{e}=\bar{e}_{0}+\dot{\bar{e}}\left(t-t_{0}\right) \\
& \bar{i}=\bar{i}_{0}+\dot{\bar{i}}\left(t-t_{0}\right) \\
& \bar{\Omega}=\bar{\Omega}_{0}+\dot{\bar{\Omega}}\left(t-t_{0}\right) \\
& \bar{\omega}=\bar{\omega}_{0}+\dot{\bar{\omega}}\left(t-t_{0}\right) \\
& \bar{M}=\bar{M}_{0}+\dot{\bar{M}}\left(t-t_{0}\right)
\end{aligned}
$$

where $\left(\bar{a}_{0}, \bar{e}_{0}, \bar{i}_{0}, \bar{\Omega}_{0}, \bar{\omega}_{0}, \bar{M}_{0}\right)^{T}$ are the initial mean classical orbit elements and $(\dot{\bar{a}}, \dot{\bar{e}}, \dot{\bar{i}}, \dot{\bar{\Omega}}, \dot{\bar{\omega}}, \dot{\bar{M}})^{T}$ are the secular variations caused by the sum of all perturbing forces. We know that the zonal harmonics of Earth gravitation potential cause secular motion in longitude of ascending node $\Omega$, argument of perigee $\omega$, and mean anomaly $M$ orbit elements. Thus the secular effects of $J_{2}$ to $J_{6}$ zonal harmonics on the classical orbit elements are expressed as :[27]

$$
\begin{aligned}
& \dot{\bar{a}}=0 \\
& \dot{\bar{e}}=0 \\
& \dot{\bar{i}}=0
\end{aligned}
$$




$$
\begin{aligned}
& \dot{\bar{\Omega}}=-\frac{3 J_{2} R_{e}^{2} \bar{n}_{0} \cos \bar{i}_{0}}{2 \bar{p}_{0}^{2}}+\frac{3 J_{2}^{2} R_{e}^{4} \bar{n}_{0} \cos \bar{i}_{0}}{32 \bar{p}_{0}^{4}}\left\{12-4 \bar{e}_{0}^{2}-\left(80+5 \bar{e}_{0}^{2}\right) \sin ^{2} \bar{i}_{0}\right\}+ \\
& \frac{15 J_{4} R_{e}^{4} \bar{n}_{0} \cos \bar{i}_{0}}{32 \bar{p}_{0}^{4}}\left\{8+12 \bar{e}_{0}^{2}-\left(14+21 \bar{e}_{0}^{2}\right) \sin ^{2} \bar{i}_{0}\right\}-\frac{105}{1024} \frac{J_{6} R_{e}^{6} \bar{n}_{0} \cos \bar{i}_{0}}{\bar{p}_{0}^{6}} \\
& \left\{64+160 \bar{e}_{0}^{2}+120 \bar{e}_{0}^{4}-\left(288+720 \bar{e}_{0}^{2}+540 \bar{e}_{0}^{4}\right) \sin ^{2} \bar{i}_{0}+\right. \\
& \left.\left(264+660 \bar{e}_{0}^{2}+495 \bar{e}_{0}^{4}\right) \sin ^{4} \bar{i}_{0}\right\} \\
& \dot{\bar{\omega}}=\frac{3 J_{2} R_{e}^{2} \bar{n}_{0}}{4 \bar{p}_{0}^{2}}\left\{4-5 \sin ^{2} \bar{i}_{0}\right\}+\frac{9 J_{2}^{2} R_{e}^{4} \bar{n}_{0}}{384 \bar{p}_{0}^{4}}\left\{56 \bar{e}_{0}^{2}+\left(760-36 \bar{e}_{0}^{2}\right) \sin ^{2} \bar{i}_{0}-\right. \\
& \left.\left(890+45 \bar{e}_{0}^{2}\right) \sin ^{4} \bar{i}_{0}\right\}-\frac{15 J_{4} R_{e}^{4} \bar{n}_{0}}{128 \bar{p}_{0}^{4}}\left\{64+72 \bar{e}_{0}^{2}-\left(248+252 \bar{e}_{0}^{2}\right) \sin ^{2} \bar{i}_{0}+\right. \\
& \left.\left(196+189 \bar{e}_{0}^{2}\right) \sin ^{4} \bar{i}_{0}\right\}+\frac{105 J_{6} R_{e}^{6} \bar{n}_{0}}{2048 \bar{p}_{0}^{6}}\left\{256+960 \bar{e}_{0}^{2}+320 \bar{e}_{0}^{4}-\right. \\
& \left(2048+6880 \bar{e}_{0}^{2}+2160 \bar{e}_{0}^{4}\right) \sin ^{2} \bar{i}_{0}+\left(4128+13080 \bar{e}_{0}^{2}+3960 \bar{e}_{0}^{4}\right) \sin ^{4} \bar{i}_{0}- \\
& \left.\left(2376+14520 \bar{e}_{0}^{2}+2145 \bar{e}_{0}^{4}\right) \sin ^{6} \bar{i}_{0}\right\} \\
& \dot{\bar{M}}=\bar{n}_{0}+\frac{3 J_{2} R_{e}^{2} \bar{n}_{0} \sqrt{1-\bar{e}_{0}^{2}}}{4 \bar{p}_{0}^{2}}\left\{2-3 \sin ^{2} \bar{i}_{0}\right\}+\frac{3 J_{2}^{2} R_{e}^{4} \bar{n}_{0}}{512 \bar{p}_{0}^{4} \sqrt{1-\bar{e}_{0}^{2}}}\left\{320 \bar{e}_{0}^{2}-280 \bar{e}_{0}^{4}+\right. \\
& \left.\left(1600-1568 \bar{e}_{0}^{2}+328 \bar{e}_{0}^{-4}\right) \sin ^{2} \bar{i}_{0}-\left(2096-1072 \bar{e}_{0}^{2}-79 \bar{e}_{0}^{4}\right) \sin ^{4} \bar{i}_{0}\right\}+ \\
& \frac{45 J_{4} R_{e}^{4} \bar{n}_{0} \bar{e}_{0}^{2} \sqrt{1-\bar{e}_{0}^{2}}}{128 \bar{p}_{0}^{4}}\left\{8-40 \sin \bar{i}_{0}+35 \sin ^{2} \bar{i}_{0}\right\}+\frac{35 J_{6} R_{e}^{6} \bar{n}_{0} \sqrt{1-\bar{e}_{0}^{2}}}{2048 \bar{p}_{0}^{6}} \\
& \left\{-128+320 \bar{e}_{0}^{2}+240 \bar{e}_{0}^{4}+\left(1344-3360 \bar{e}_{0}^{2}-2520 \bar{e}_{0}^{4}\right) \sin \bar{i}_{0}+\right. \\
& \left.\left(-1512+3780 \bar{e}_{0}^{2}+2835 \bar{e}_{0}^{4}\right) \sin ^{2} \bar{i}_{0}+\left(1848-4620 \bar{e}_{0}^{2}-3465 \bar{e}_{0}^{4}\right) \sin ^{4} \bar{i}_{0}\right\}
\end{aligned}
$$

\subsubsection{Simulation Test Plans}

We devised three different simulation test cases for evaluating the precision of GimAlfriend closed-form solution. In each test case the relative motions of two deputy satellites with respect to a chief satellite are examined. In test cases 1 and 2 , the chief and two deputy satellites fly in close formation and in test case 3 , a far formation flight of satellites is investigated. These test cases together can effectively assess the accuracy of Gim-Alfriend closed-form solution for most common satellites 
formation flying applications such as Earth surveillance, remote sensing, and spacebased radar. Each test case simulation is executed for a duration of 15 chief satellite orbit periods. We will observe that the simulated flight times of 15 orbits is long enough for each test case to reveal any subtle secular increase in prediction errors. The numerical integrations of both chief and deputy satellites equations of motions in the ECI reference frame is performed in 0.5 seconds time steps. The computation of the predicted relative motion of the deputy satellites in the chief LVLH curvilinear frame via Gim-Alfriend state transition matrix is also performed in 0.5 seconds time step. This allows each test case to measure very precisely the prediction errors of the Gim-Alfriend closed-form solution. The initial conditions of the chief and deputy satellites for each test case are given in form of the classical orbit elements $\left(a_{0}, e_{0}, i_{0}, \Omega_{0}, \omega_{0}, M_{0}\right)^{T}$ and $\left(\delta a_{0}, \delta e_{0}, \delta i_{0}, \delta \Omega_{0}, \delta \omega_{0}, \delta M_{0}\right)^{T}$ respectively. Thus, the deputy satellite initial relative orbit elements $\delta \boldsymbol{e}=\left(\delta a_{0}, \delta \theta_{0}, \delta i_{0}, \delta q_{10}, \delta q_{20}, \delta \Omega_{0}\right)^{T}$ are determined from the initial classical relative orbit elements using the following identities

$$
\begin{aligned}
\delta \theta_{0} & =\delta \omega_{0}+\delta f_{0} \\
\delta q_{10} & =\delta e_{0} \cos \omega_{0}-e_{0} \sin \omega_{0} \delta \omega_{0} \\
\delta q_{20} & =\delta e_{0} \sin \omega_{0}+e_{0} \cos \omega_{0} \delta \omega_{0}
\end{aligned}
$$

Moreover, the initial relative state vector $\boldsymbol{X}_{0}$ of each deputy satellite is obtained using equation (2.53). 


\subsubsection{Satellites Close Formation Flight Test Case 1}

The initial conditions for this test case are chosen such that the projection of the relative orbit of each deputy satellite in the chief local horizontal plane is near circular with the chief satellite located at the center. The radii of these projected near circular relative orbits will be less than 600 meters. The chief satellite will have an eccentricity of 0.005 which will generate a slight in-track drift in the relative motions of deputy satellites, even if there are no perturbations ${ }^{[7]}$. Table 2.1 and 2.2 describe the initial conditions for the chief and two deputy satellites in test case 1.

\begin{tabular}{|l|c|c|c|c|c|c|}
\hline \multirow{2}{*}{ Chief Satellite } & $a_{0}(\mathrm{~m})$ & $e_{0}$ & $i_{0}(\mathrm{deg})$ & $\Omega_{0}(\mathrm{deg})$ & $\omega_{0}(\mathrm{deg})$ & $M_{0}(\mathrm{deg})$ \\
\cline { 2 - 7 } & $7100 \mathrm{e} 3$ & 0.005 & 70 & 45 & 20 & 29.7144 \\
\hline \multirow{2}{*}{ Deputy Satellite 1 } & $\delta a_{0}(\mathrm{~m})$ & $\delta e_{0}$ & $\delta i_{0}(\mathrm{deg})$ & $\delta \Omega_{0}(\mathrm{deg})$ & $\delta \omega_{0}(\mathrm{deg})$ & $\delta M_{0}(\mathrm{deg})$ \\
\cline { 2 - 7 } & -1.272 & $1.7 \mathrm{e}-5$ & $2.6 \mathrm{e}-3$ & $3.2 \mathrm{e}-3$ & -0.3473 & 0.3462 \\
\hline
\end{tabular}

Table 2.1: Test case 1 initial conditions for the chief and deputy satellite 1

\begin{tabular}{|l|c|c|c|c|c|c|}
\hline \multirow{2}{*}{ Chief Satellite } & $a_{0}(\mathrm{~m})$ & $e_{0}$ & $i_{0}(\mathrm{deg})$ & $\Omega_{0}(\mathrm{deg})$ & $\omega_{0}(\mathrm{deg})$ & $M_{0}(\mathrm{deg})$ \\
\cline { 2 - 7 } & $7100 \mathrm{e} 3$ & 0.005 & 70 & 45 & 20 & 159.8033 \\
\hline \multirow{2}{*}{ Deputy Satellite 2} & $\delta a_{0}(\mathrm{~m})$ & $\delta e_{0}$ & $\delta i_{0}(\mathrm{deg})$ & $\delta \Omega_{0}(\mathrm{deg})$ & $\delta \omega_{0}(\mathrm{deg})$ & $\delta M_{0}(\mathrm{deg})$ \\
\cline { 2 - 7 } & -0.839 & $1.2 \mathrm{e}-5$ & $-4.05 \mathrm{e}-3$ & 0 & 0.3822 & -0.3820 \\
\hline
\end{tabular}

Table 2.2: Test case 1 initial conditions for the chief and deputy satellite 2

We run the test case 1 simulation for a duration of 15 chief orbit periods which is equal to 89310 seconds or almost 24.81 hours. The orbit periods for the deputy satellites however, are slightly smaller because we set the initial semi-major axis difference $\delta a_{0}$ to -1.272 and -0.839 meters for the deputy satellites 1 and 2 respectively. We expect that any $\delta a_{0} \neq 0$ will add more in-track drift to the relative motion of the associated deputy satellite even in the absence of any perturbations. Figure 2.6 displays the chief and deputy satellites inertial and relative orbit trajectories for the test case 1 . Figure 2.6 a shows that a chief and two deputy satellites fly in formation 
at an altitude of almost 720 kilometers and in orbits with next to 70 degrees inclination. Figure 2.6b exhibits a beautifully created relative orbit trajectories for the two deputy satellites. The big purple circle in the middle of Figure 2.6a depicts Earth and it is not to scale. The small purple square in the middle of Figure $2.6 \mathrm{~b}$ represents the chief satellite. The maximum range distance between the chief satellite and any of the two deputy satellites is less than 800 meters. 


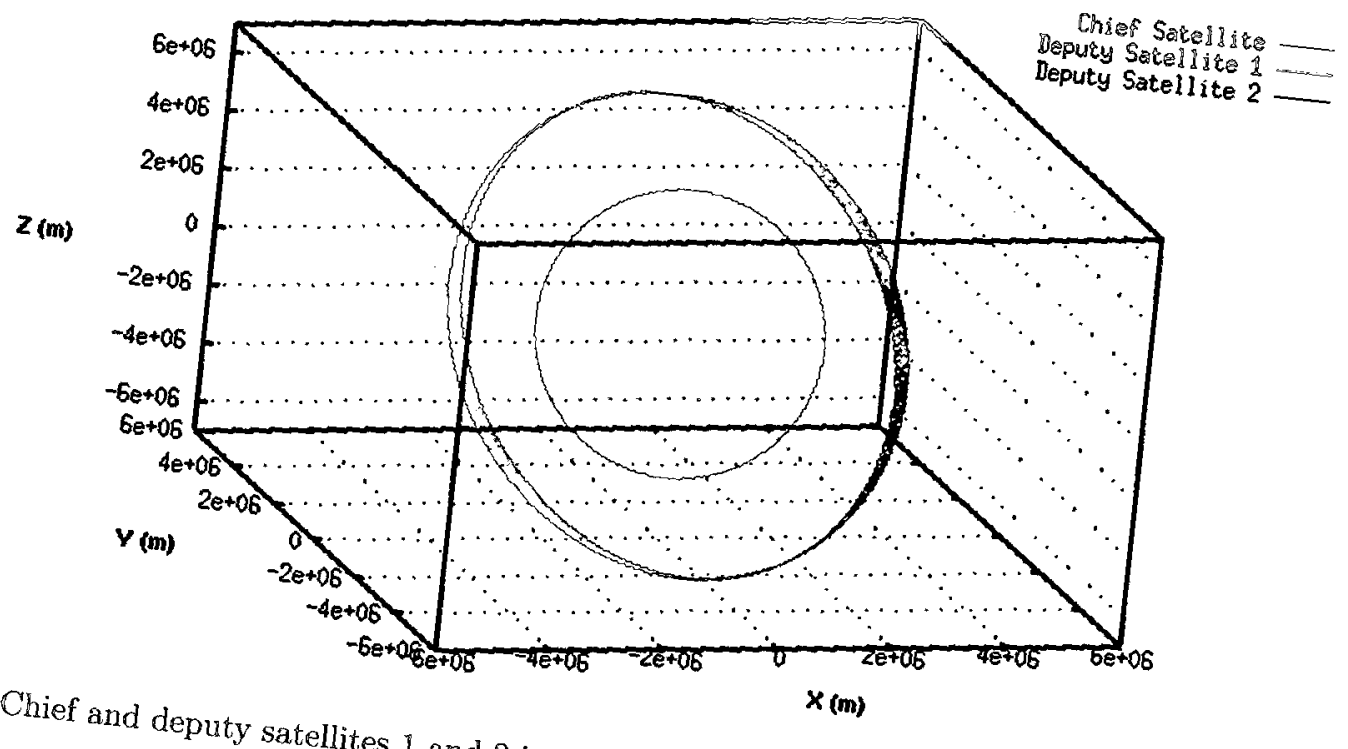

(a) Chief and deputy satellites 1 and 2 inertial orbit trajectories in the ECI reference frame

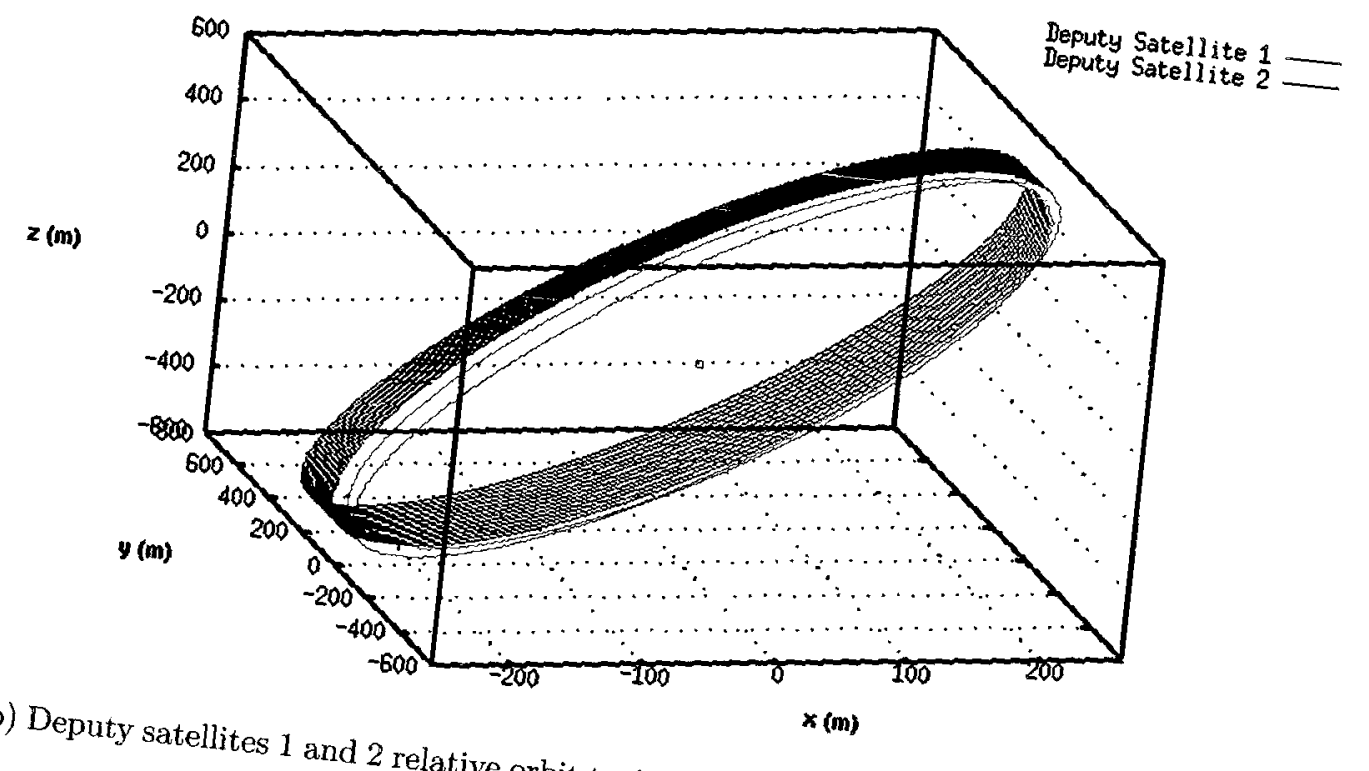

Figure 2.6: Illustration of the chief and trajectories in the chief LVLH rectilinear frame trajectories in the test case 1 chief and deputy satellites' inertial and relative 


\section{Simulation results for the deputy satellite 1}

Figure 2.7 displays the deputy satellite 1 actual relative position and velocity vector components in the chief LVLH curvilinear frame and compare it against the predicted relative position and velocity vectors components that are produced by the Gim-Alfriend close-form solution. Figure 2.8 demonstrates the performance of GimAlfriend analytical method in accomplishing the relative orbit prediction task for the deputy satellite 1 . Figure 2.8 a shows that the maximum absolute value of prediction errors for the deputy satellite 1 relative position vector components belongs to in-track element and is less than 3 meters for the entire simulated flight times. We also notice that there is a prominent secular growth in the prediction error for the in-track relative position vector component. The prediction errors for the radial and cross-track elements however, exhibit a very small secular change. It is clear that for the deputy satellite 1 , the size of the relative orbit is small and therefore the $J_{2}$ gravitational perturbation in comparison with the $J_{3}$ to $J_{6}$ gravitational disturbances must play a dominant role in producing periodic oscillations in the chief satellite and deputy satellite 1 osculating orbit elements. This fact suggests that the conspicuous in-track secular growth has mainly resulted from the neglected higher order terms of $J_{2}$ gravitational perturbation in the Gim-Alfriend state transition matrix. Turning our attention to Figure $2.8 \mathrm{~b}$, we note that the maximum absolute value of prediction errors for the deputy satellite 1 relative velocity vector components is less 2.2 millimeters per second and there are indications of very small secular change in the prediction errors during 24.81 hours of simulated flight times.

We infer from these test case 1 simulation results that the Gim-Alfriend closedform solution provides very good predictions for the deputy satellite 1 relative position and velocity vectors. 

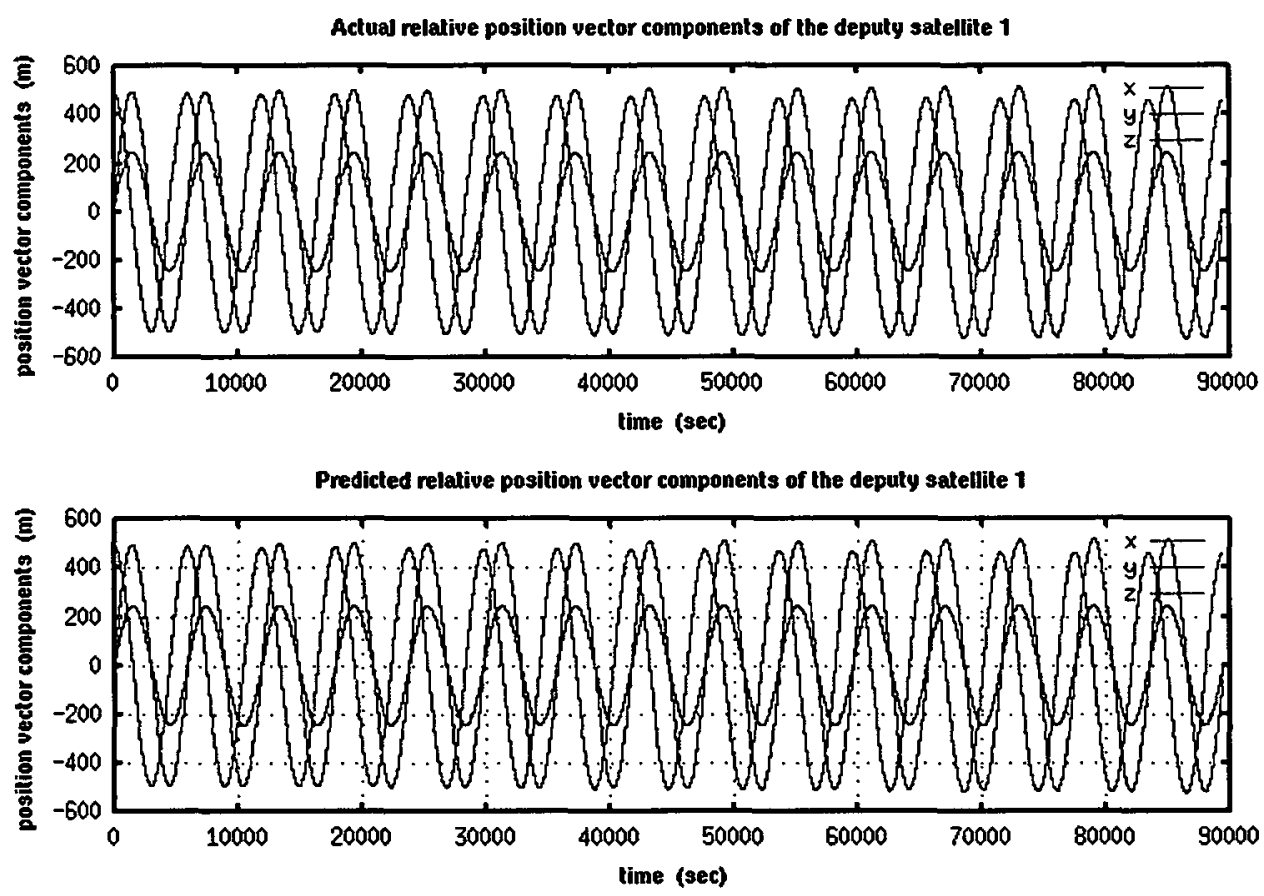

(a) Actual and predicted relative position vectors components of the deputy satellite 1
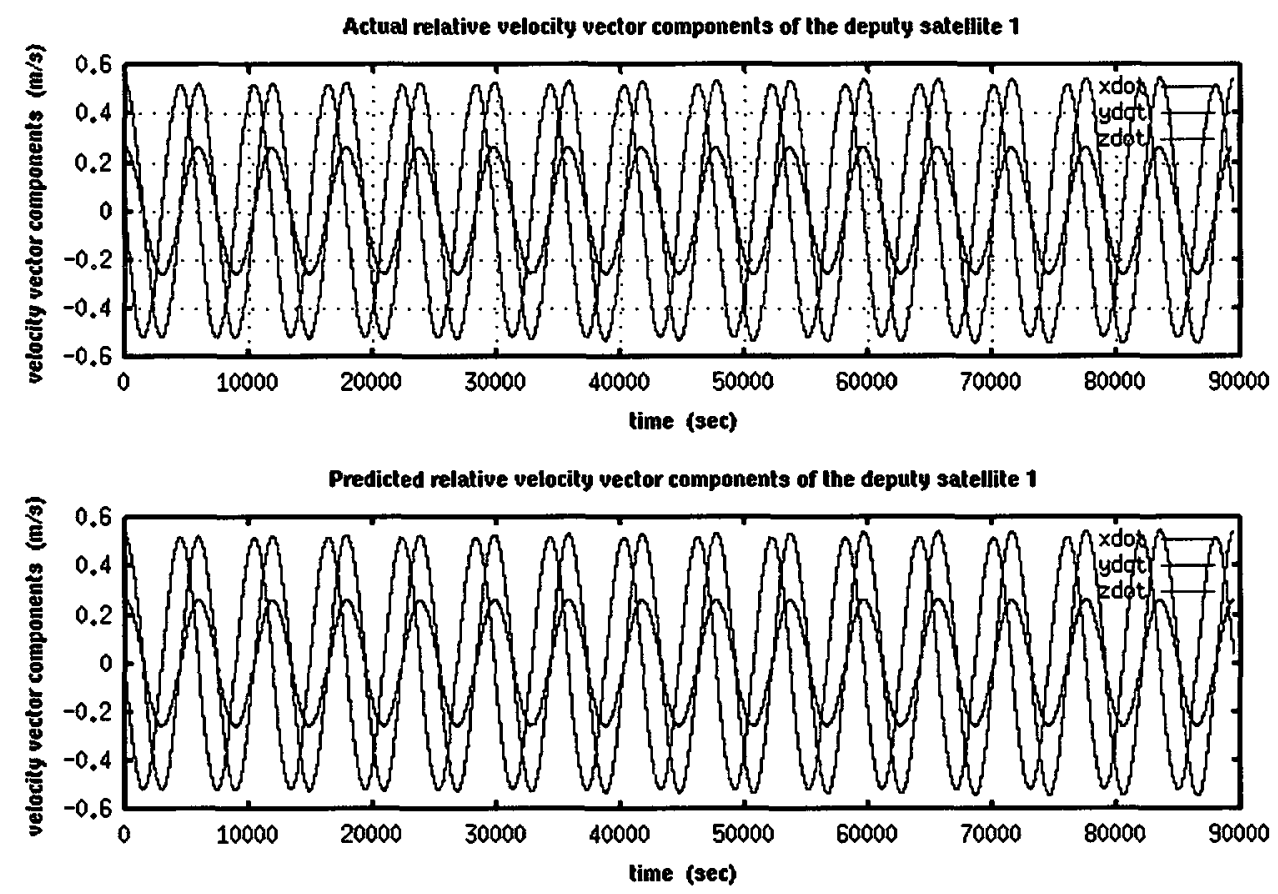

(b) Actual and predicted relative velocity vectors components of the deputy satellite 1

Figure 2.7: Illustration of the deputy satellite 1 actual/predicted relative position and velocity vectors components in the test case 1 


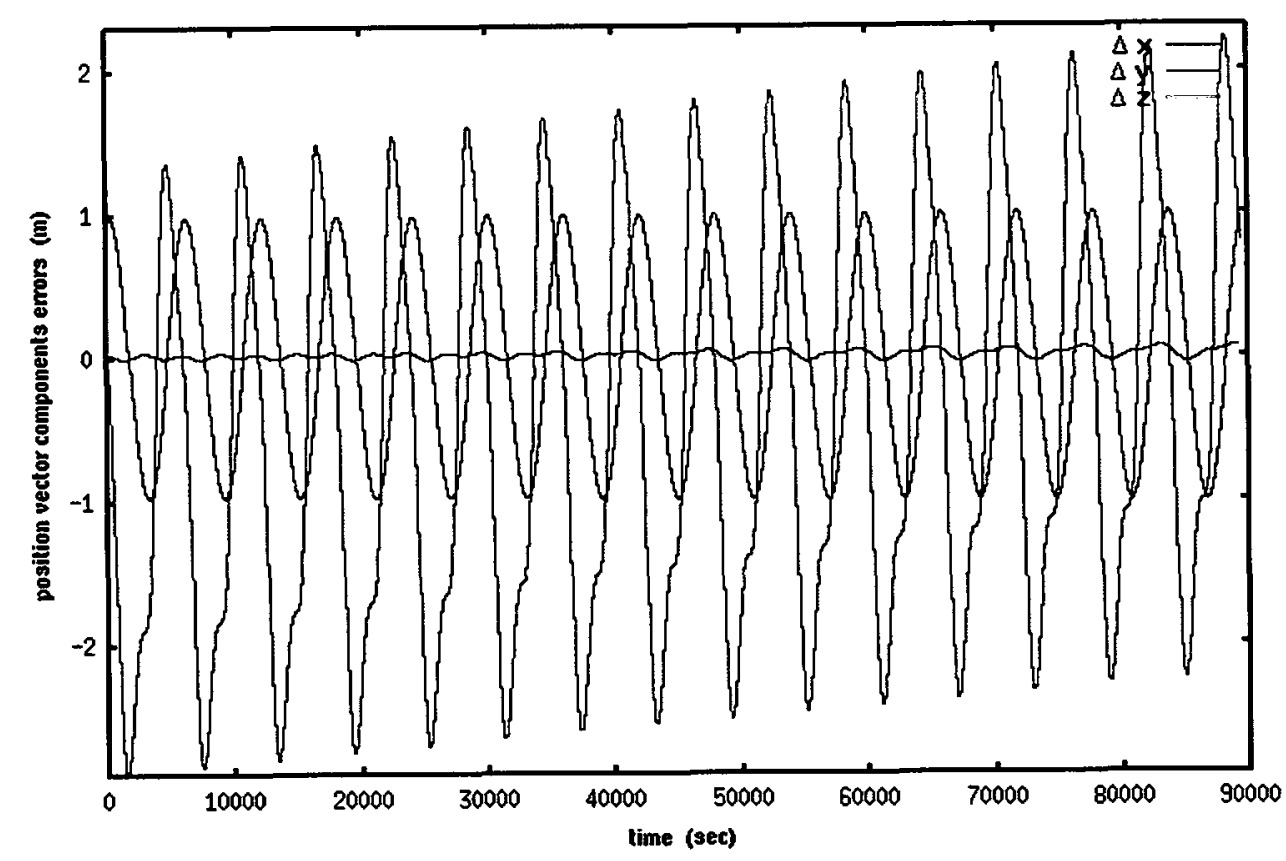

(a) Relative position vector components errors of the deputy satellite 1

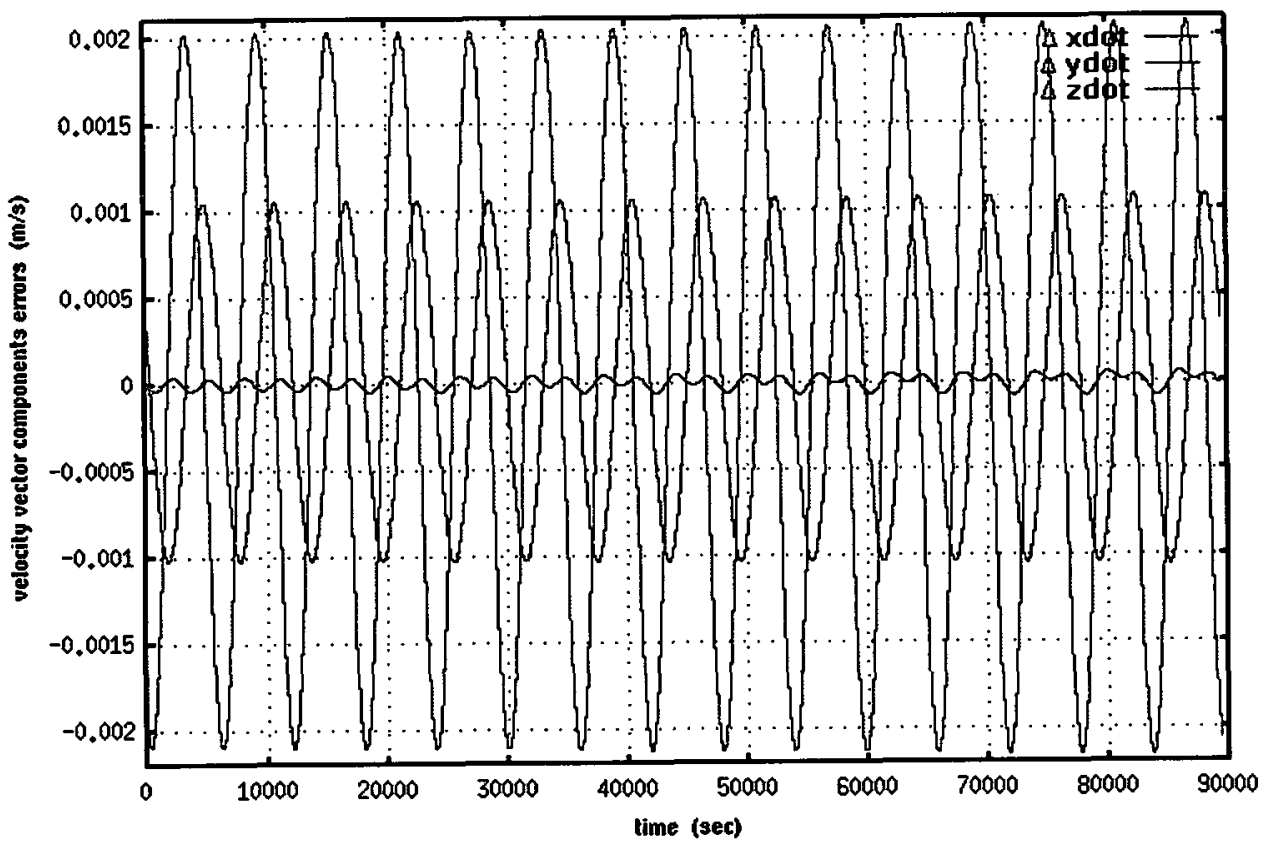

(b) Relative velocity vector components errors of the deputy satellite 1

Figure 2.8: Illustration of the deputy satellite 1 relative position and velocity errors in the test case 1 


\section{Simulation results for the deputy satellite 2}

Figure 2.9 displays the deputy satellite 2 actual relative position and velocity vector components in the chief LVLH curvilinear frame and compare it against the predicted relative position and velocity vectors components that are produced by the Gim-Alfriend close-form solution. Figure 2.10 demonstrates the performance of GimAlfriend analytical method in accomplishing the relative orbit prediction task for the deputy satellite 2. Figure $2.10 \mathrm{a}$ shows that the maximum absolute value of prediction errors for the deputy satellite 2 relative position vector components belongs to in-track element and is less than 3 meters for the entire simulated flight times. We also notice that there is a prominent secular growth in the prediction error for the in-track relative position vector component. The prediction errors for the radial and cross-track elements however, exhibit a very small secular change. It is clear that for the deputy satellite 2, the size of the relative orbit is small and therefore the $J_{2}$ gravitational perturbation in comparison with the $J_{3}$ to $J_{6}$ gravitational disturbances must play a dominant role in producing periodic oscillations in the chief satellite and deputy satellite 2 osculating orbit elements. This fact suggests that the in-track secular growth has mainly resulted from the neglected higher order terms of $J_{2}$ gravitational perturbation in the Gim-Alfriend state transition matrix. Turning our attention to Figure 2.10b, we note that the maximum absolute value of prediction errors for the deputy satellite 2 relative velocity vector components is less 2.2 millimeters per second and there are small secular changes present in the prediction errors during 24.81 hours of simulated flight times.

We infer from these test case 1 simulation results that the Gim-Alfriend closedform solution provides very good predictions for the deputy satellite 2 relative position and velocity vectors. 

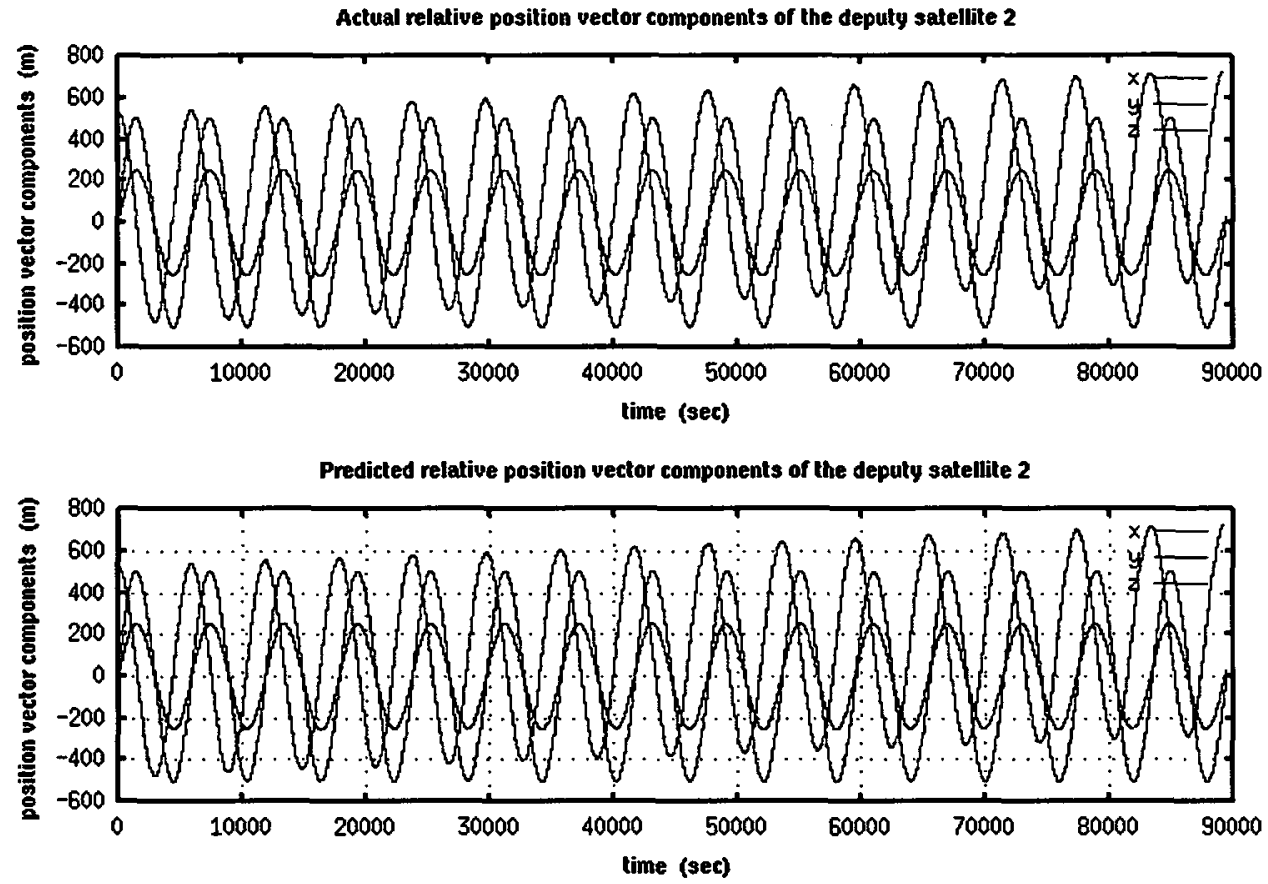

(a) Actual and predicted relative position vectors components of the deputy satellite 2
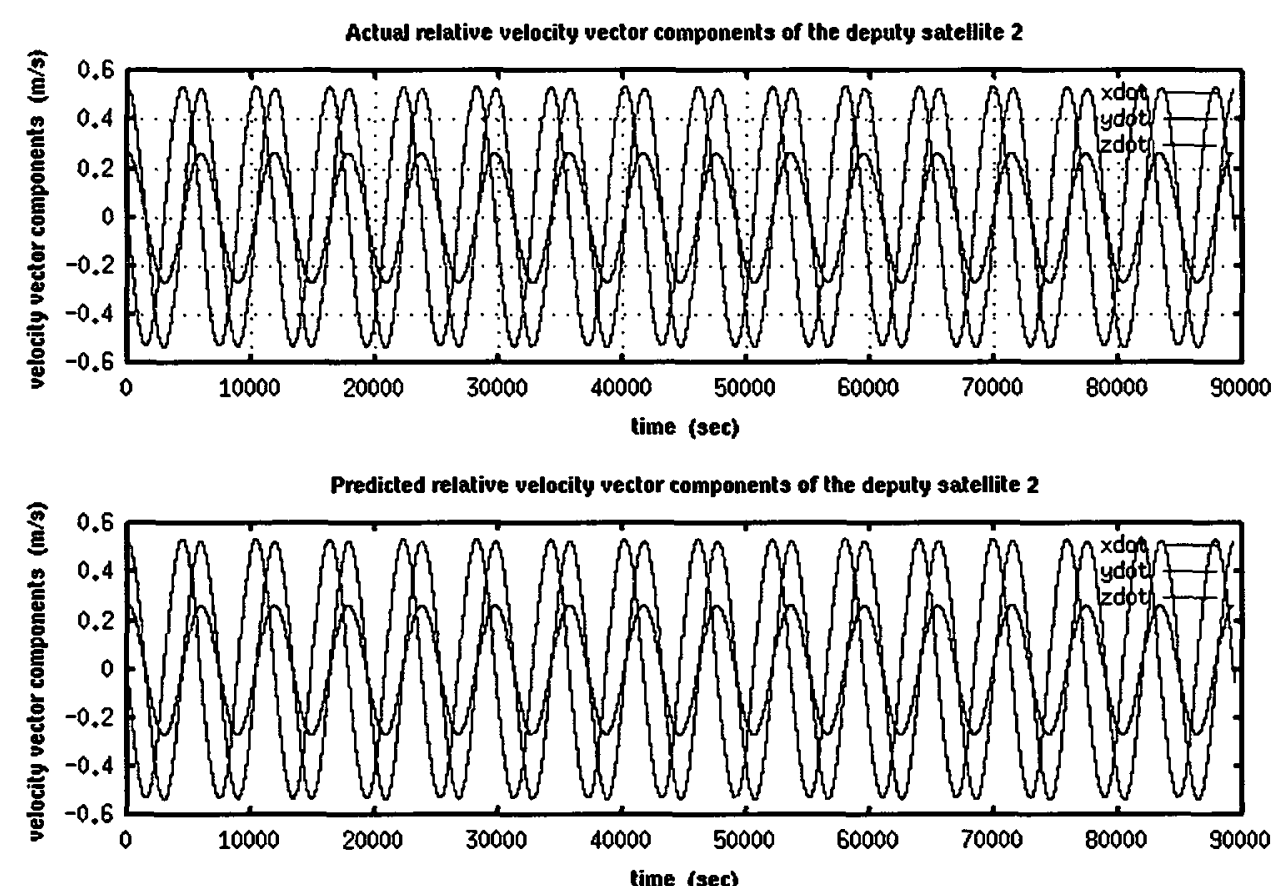

(b) Actual and predicted relative velocity vectors components of the deputy satellite 2

Figure 2.9: Illustration of the deputy satellite 2 actual/predicted relative position and velocity vectors components in the test case 1 


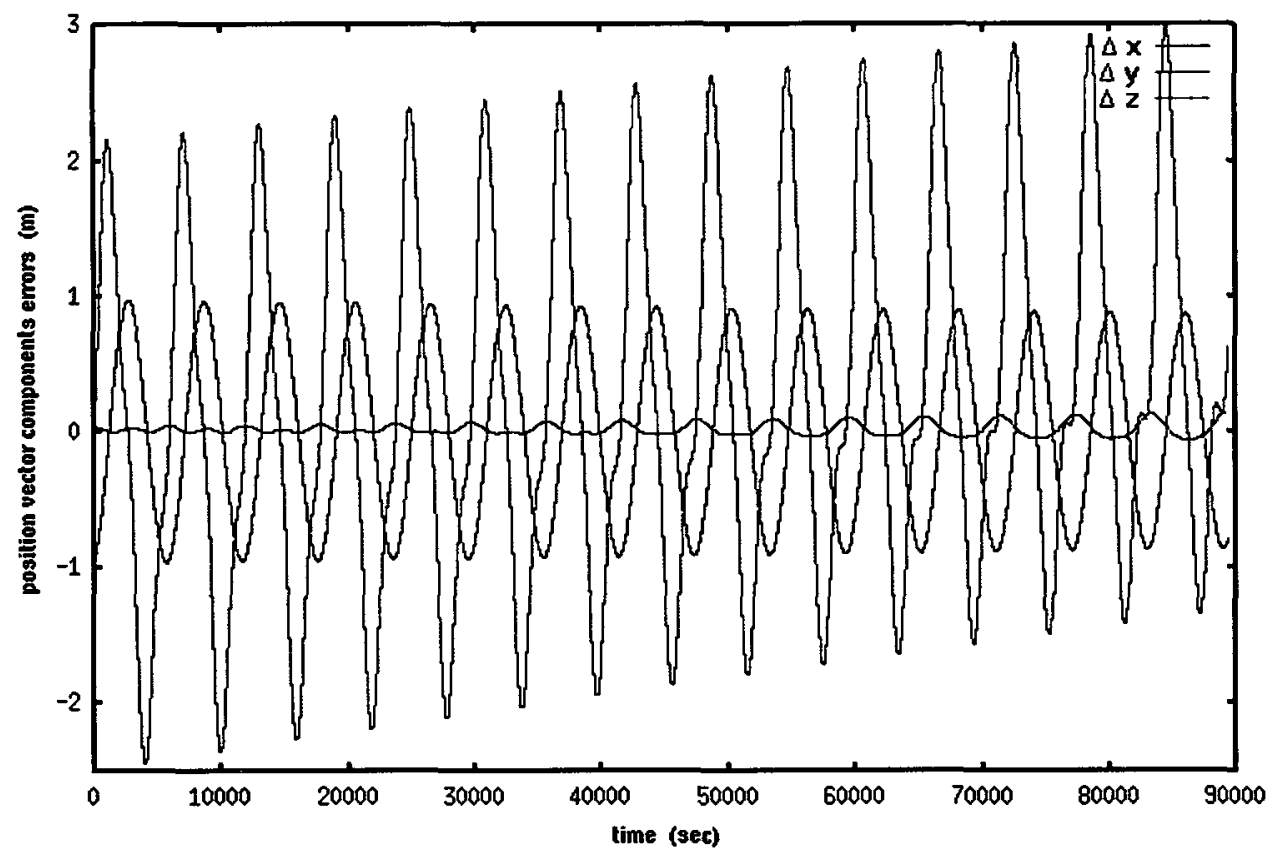

(a) Relative position vector components errors of the deputy satellite 2

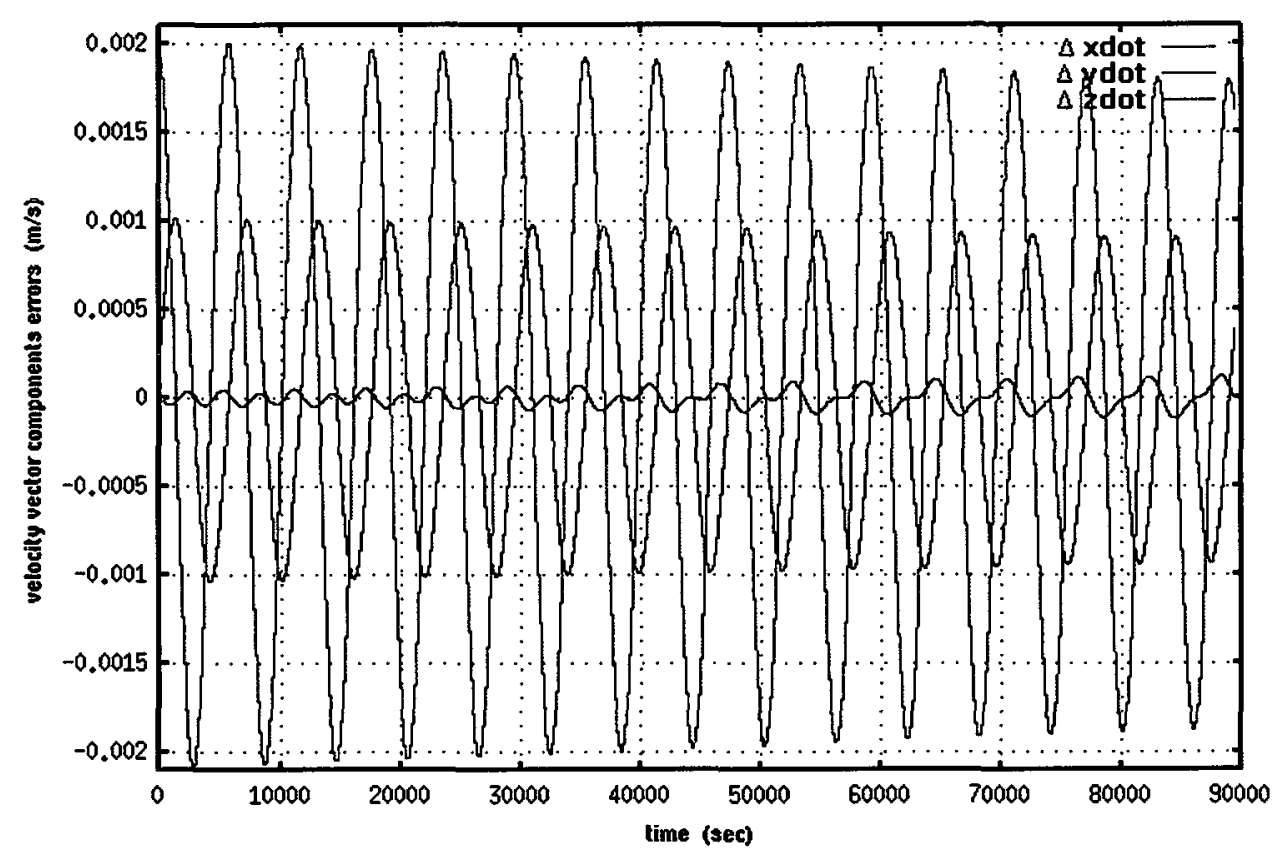

(b) Relative velocity vector components errors of the deputy satellite 2

Figure 2.10: Illustration of the deputy satellite 2 relative position and velocity errors in the test case 1 


\subsubsection{Satellites Close Formation Flight Test Case 2}

The initial conditions for this test case are chosen such that the projection of the relative orbit of each deputy satellite in the chief local horizontal plane is quasi circular with the chief satellite located close to the center. The radii of these projected quasi circular relative orbits will be less than 700 meters. The chief satellite will have an eccentricity of 0.005 which will generate a slight in-track drift in the relative motions of deputy satellites, even if there are no perturbations. Table 2.3 and 2.4 describe the initial conditions for the chief and two deputy satellites in test case 2 .

\begin{tabular}{|l|c|c|c|c|c|c|}
\hline \multirow{2}{*}{ Chief Satellite } & $a_{0}(\mathrm{~m})$ & $e_{0}$ & $i_{0}(\mathrm{deg})$ & $\Omega_{0}(\mathrm{deg})$ & $\omega_{0}(\mathrm{deg})$ & $M_{0}(\mathrm{deg})$ \\
\cline { 2 - 7 } & $6800 \mathrm{e} 3$ & 0.005 & 20 & 10 & 25 & 29.7144 \\
\hline \multirow{2}{*}{ Deputy Satellite 1} & $\delta a_{0}(\mathrm{~m})$ & $\delta e_{0}$ & $\delta i_{0}(\mathrm{deg})$ & $\delta \Omega_{0}(\mathrm{deg})$ & $\delta \omega_{0}(\mathrm{deg})$ & $\delta M_{0}(\mathrm{deg})$ \\
\cline { 2 - 7 } & -1.272 & $1.7 \mathrm{e}-5$ & $2.6 \mathrm{e}-3$ & $3.2 \mathrm{e}-3$ & -0.3473 & 0.3462 \\
\hline
\end{tabular}

Table 2.3: Test case 2 initial conditions for the chief and deputy satellite 1

\begin{tabular}{|l|c|c|c|c|c|c|}
\hline \multirow{2}{*}{ Chief Satellite } & $a_{0}(\mathrm{~m})$ & $e_{0}$ & $i_{0}(\mathrm{deg})$ & $\Omega_{0}(\mathrm{deg})$ & $\omega_{0}(\mathrm{deg})$ & $M_{0}(\mathrm{deg})$ \\
\cline { 2 - 7 } & $6800 \mathrm{e} 3$ & 0.005 & 20 & 10 & 25 & 159.8033 \\
\hline \multirow{2}{*}{ Deputy Satellite 2} & $\delta a_{0}(\mathrm{~m})$ & $\delta e_{0}$ & $\delta i_{0}(\mathrm{deg})$ & $\delta \Omega_{0}(\mathrm{deg})$ & $\delta \omega_{0}(\mathrm{deg})$ & $\delta M_{0}(\mathrm{deg})$ \\
\cline { 2 - 7 } & -0.839 & $1.2 \mathrm{e}-5$ & $-4.05 \mathrm{e}-3$ & 0 & 0.3822 & -0.3820 \\
\hline
\end{tabular}

Table 2.4: Test case 2 initial conditions for the chief and deputy satellite 2

We run the test case 2 simulation for a duration of 15 chief orbit periods which is equal to 83715 seconds or almost 23.25 hours. The orbit periods for the deputy satellites however, are slightly smaller because we set the initial semi-major axis difference $\delta a_{0}$ to -1.272 and -0.839 meters for the deputy satellites 1 and 2 respectively. We expect that any $\delta a_{0} \neq 0$ will add more in-track drift to the relative motion of the associated deputy satellite even in the absence of any perturbations. Figure 2.11 displays the chief and deputy satellites inertial and relative orbit trajectories for the test case 2 . Figure 2.11a shows that a chief and two deputy satellites fly in formation 
at an altitude of almost 420 kilometers and in orbits with close to 20 degrees inclination. Figure $2.11 \mathrm{~b}$ exhibits the created relative orbit trajectories for the two deputy satellites. The big purple circle in the middle of Figure 2.11a depicts Earth and it is not to scale. The small purple square in the middle of Figure $2.11 \mathrm{~b}$ represents the chief satellite. The maximum range distance between the chief satellite and any of the two deputy satellites is less than 880 meters. 


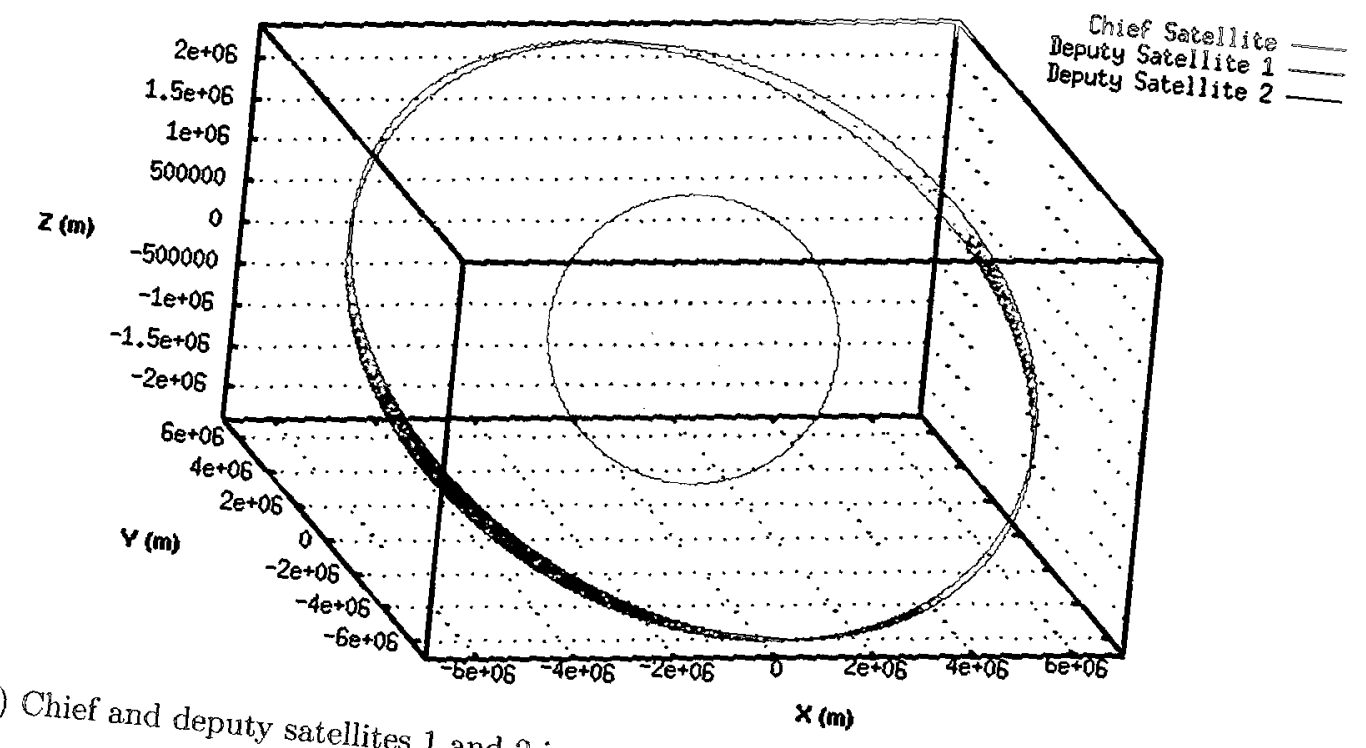

(a) Chief and deputy satellites 1 and 2 (m)

inertial orbit trajectories in the $\mathbb{E C I}$ reference frame

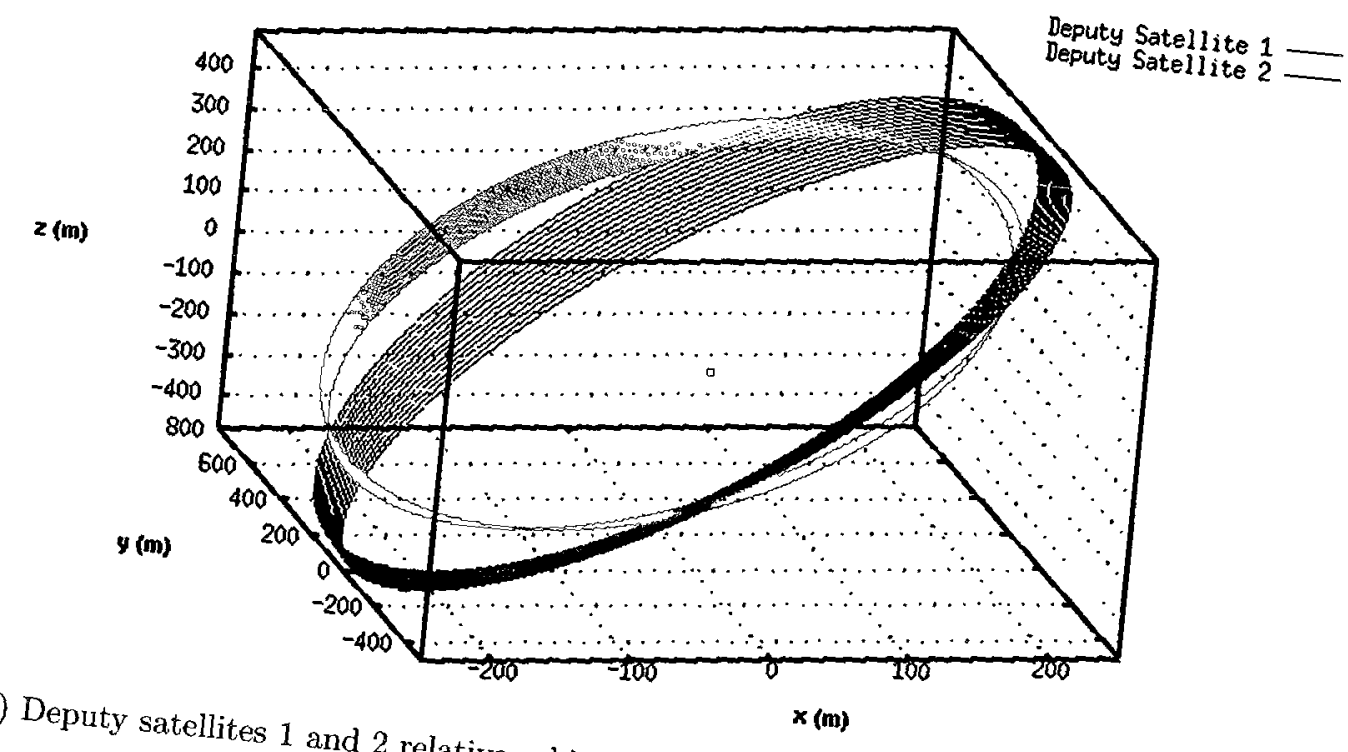

Figure 2.11: Illustration of the chief and depulectories in the chief LVLH rectilinear frame trajectories in the test case 2 chief and deputy satellites' inertial and relative 


\section{Simulation results for the deputy satellite 1}

Figure 2.12 displays the deputy satellite 1 actual relative position and velocity vector components in the chief LVLH curvilinear frame and compare it against the predicted relative position and velocity vectors components that are produced by the Gim-Alfriend close-form solution. Figure 2.13 demonstrates the performance of GimAlfriend analytical method in accomplishing the relative orbit prediction task for the deputy satellite 1 . Figure 2.13 a shows that the maximum absolute value of prediction errors for the deputy satellite 1 relative position vector components belongs to in-track element and is less than 2.5 meters for the entire simulated flight times. We also notice that there is a prominent secular growth in the prediction error for the in-track relative position vector component. The prediction errors for the radial and cross-track elements however, exhibit somewhat smaller secular changes. It must be clear to us that the conspicuous in-track secular growth has resulted from the neglected higher order terms of $J_{2}$ gravitational perturbation in the Gim-Alfriend state transition matrix. Turning our attention to Figure $2.13 \mathrm{~b}$, we note that the maximum absolute value of prediction errors for the deputy satellite 1 relative velocity vector components is less 2.2 millimeters per second and there are indications of secular changes in the prediction errors during 23.25 hours of simulated flight times.

We infer from these test case 2 simulation results that the Gim-Alfriend closedform solution provides very good predictions for the deputy satellite 1 relative position and velocity vectors. 

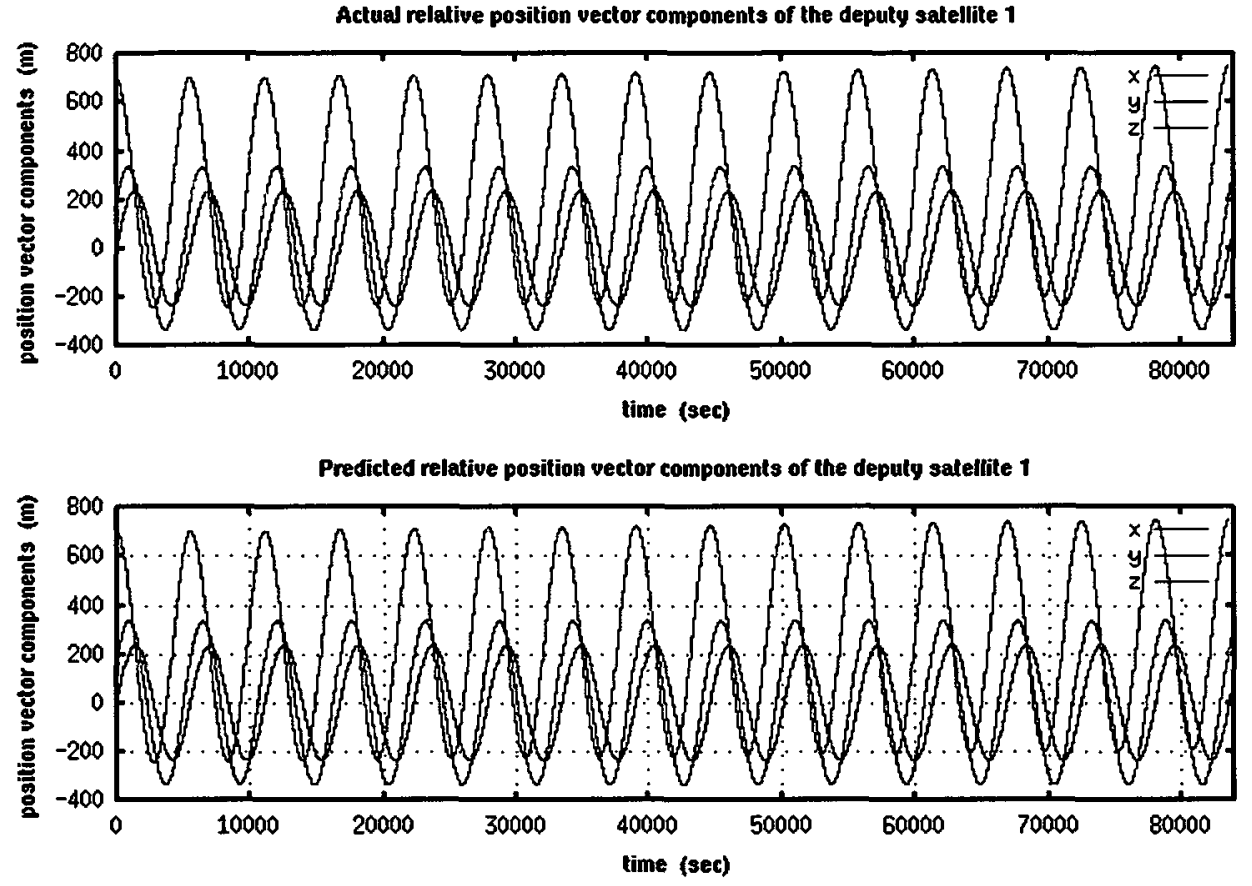

(a) Actual and predicted relative position vectors components of the deputy satellite 1


(b) Actual and predicted relative velocity vectors components of the deputy satellite 1

Figure 2.12: Illustration of the deputy satellite 1 actual/predicted relative position and velocity vectors components in the test case 2 


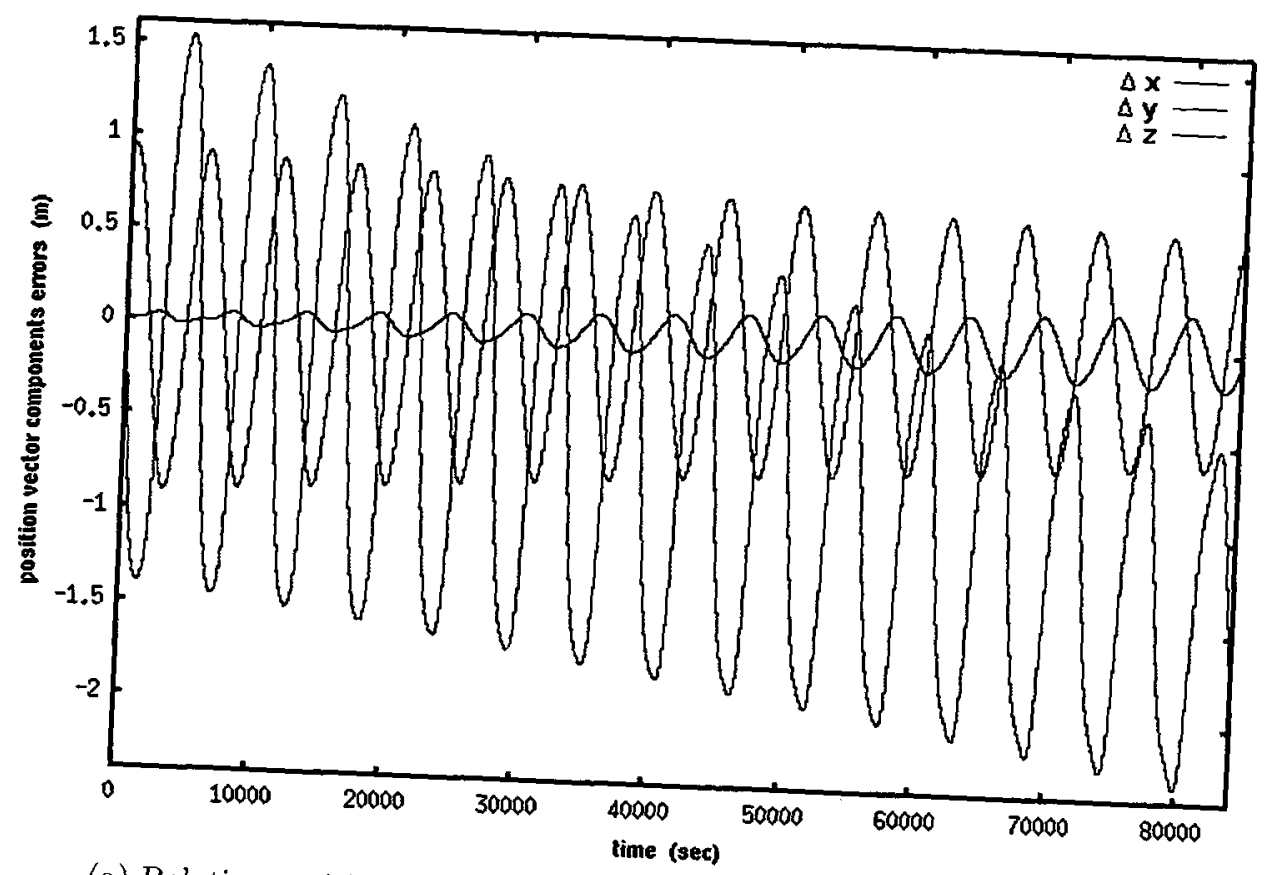

(a) Relative position vector components errors of the deputy satellite 1

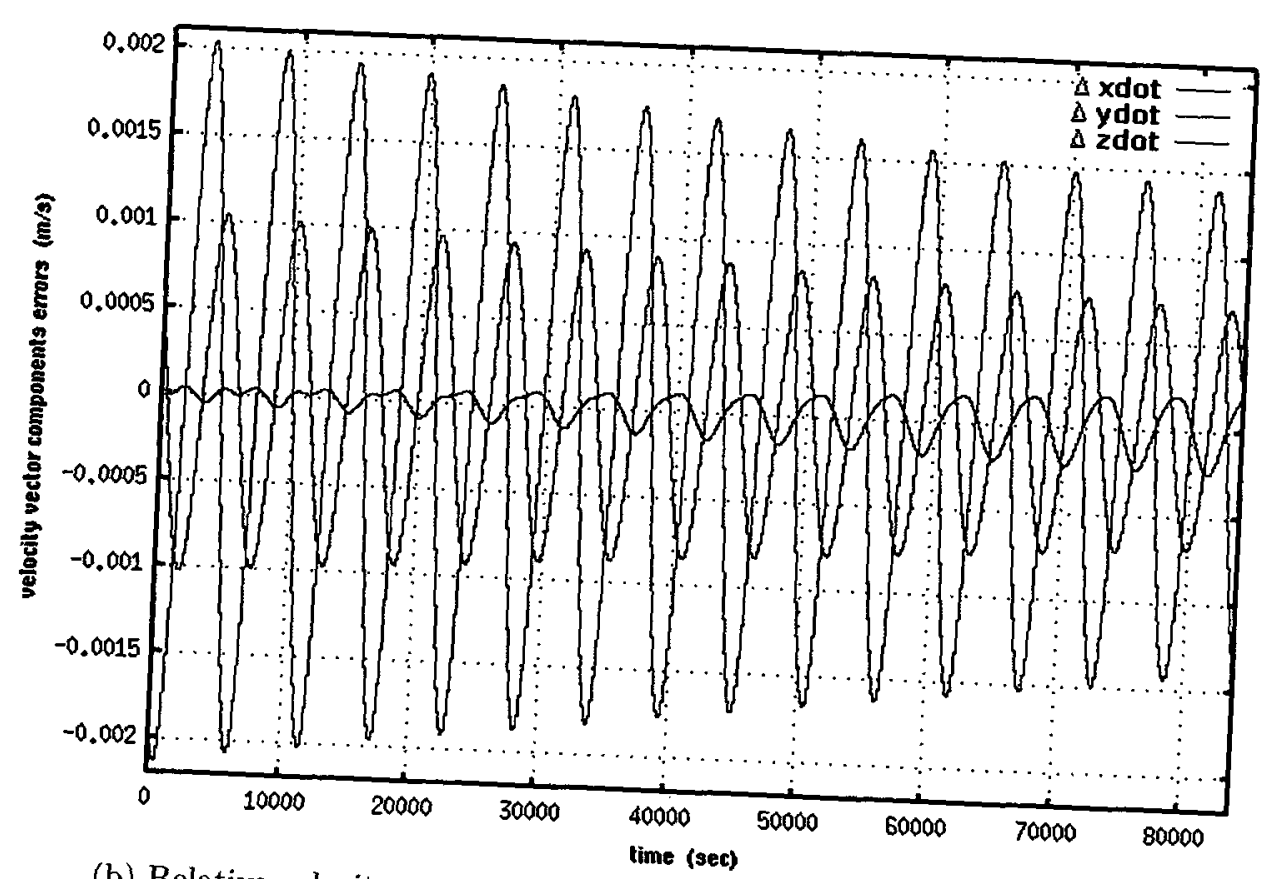

(b) Relative velocity vector components errors of the deputy satellite 1

Figure 2.13: Illustration of the deputy satellite 1 relative position and velocity errors 


\section{Simulation results for the deputy satellite 2}

Figure 2.14 displays the deputy satellite 2 actual relative position and velocity vector components in the chief LVLH curvilinear frame and compare it against the predicted relative position and velocity vectors components that are produced by the Gim-Alfriend close-form solution. Figure 2.15 demonstrates the performance of GimAlfriend analytical method in accomplishing the relative orbit prediction task for the deputy satellite 2. Figure 2.15a shows that the maximum absolute value of prediction errors for the deputy satellite 2 relative position vector components belongs to in-track element and is about 4 meters for the entire simulated flight times. We also notice that there is a prominent secular growth in the prediction error for the in-track relative position vector component. The prediction errors for the radial and cross-track elements also exhibit small secular changes. It is clear that the in-track, radial, and cross-track secular growths have resulted primarily from the neglected higher order terms of $J_{2}$ gravitational perturbation in the Gim-Alfriend state transition matrix. Turning our attention to Figure 2.15b, we note that the maximum absolute value of prediction errors for the deputy satellite 2 relative velocity vector components is about 3 millimeters per second and there are salient secular changes present in the prediction errors during 23.25 hours of simulated flight times.

We infer from these test case 2 simulation results that the Gim-Alfriend closedform solution provides good predictions for the deputy satellite 2 relative position and velocity vectors. 

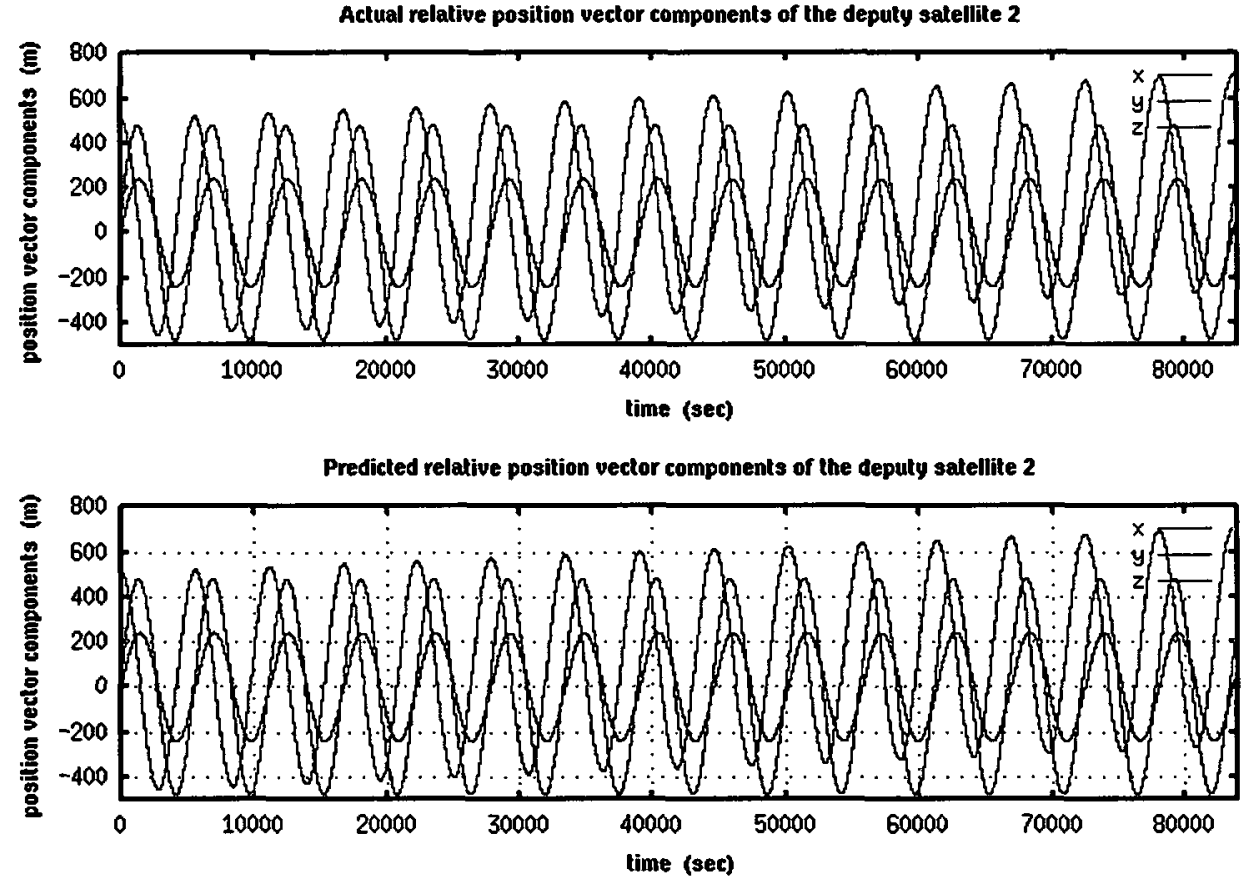

(a) Actual and predicted relative position vectors components of the deputy satellite 2
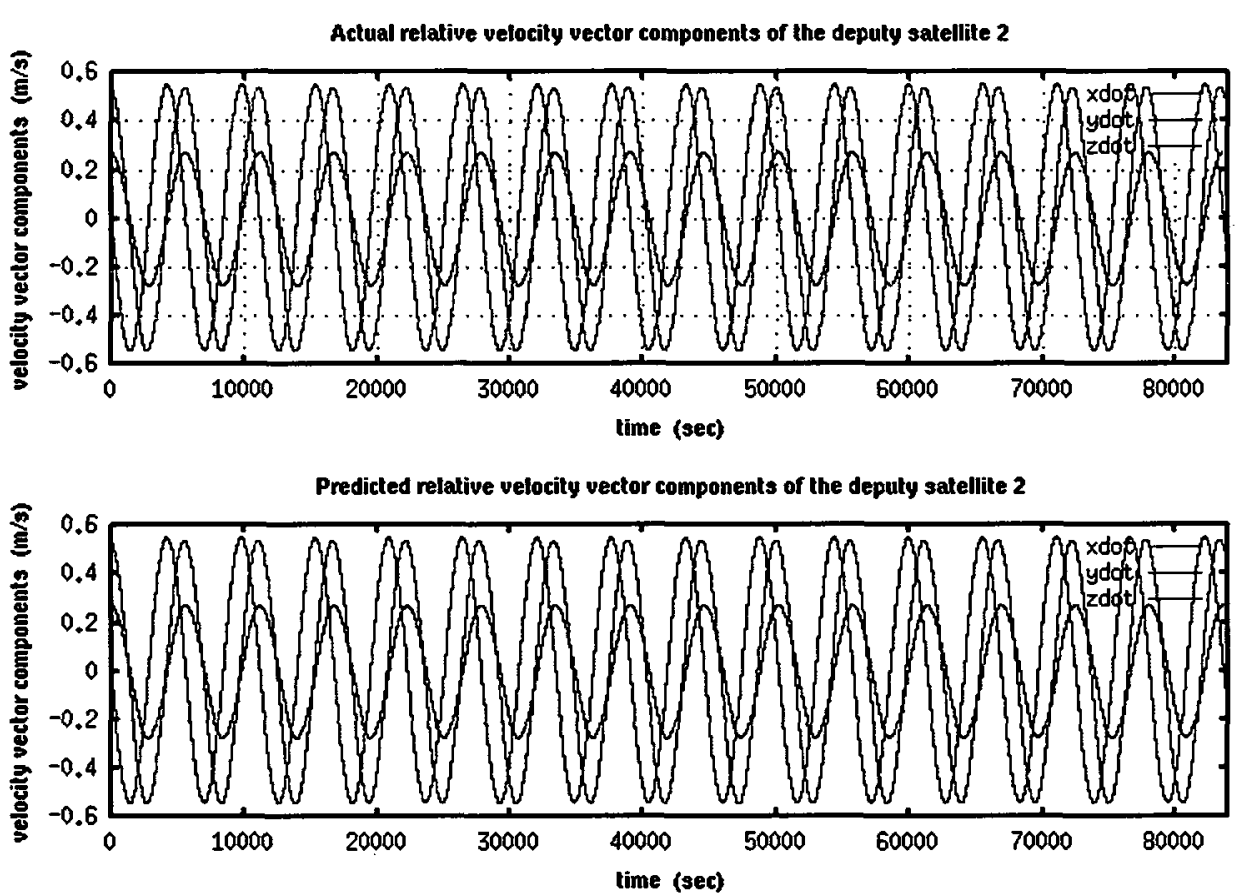

(b) Actual and predicted relative velocity vectors components of the deputy satellite 2

Figure 2.14: Illustration of the deputy satellite 2 actual/predicted relative position and velocity vectors components in the test case 2 


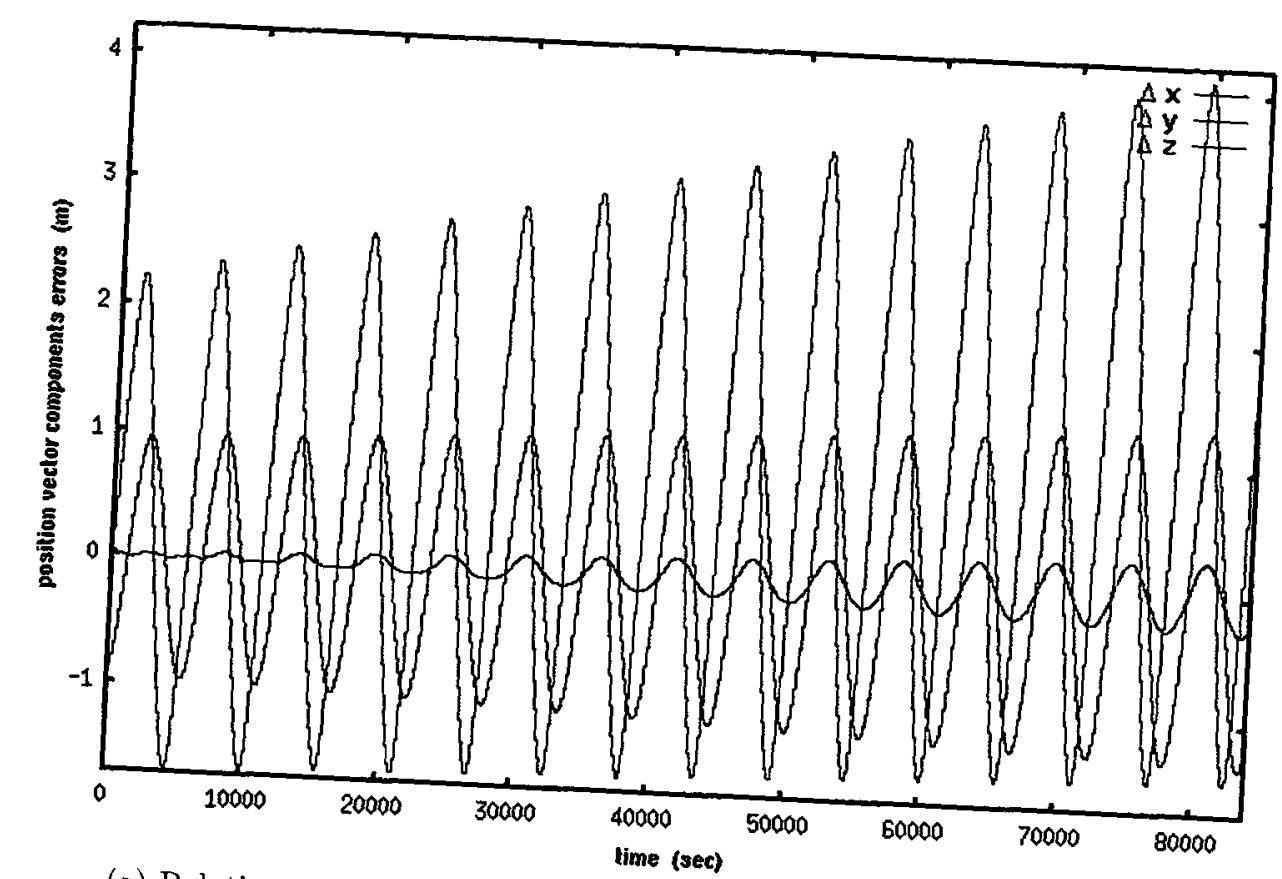

(a) Relative position vector components errors of the deputy satellite 2

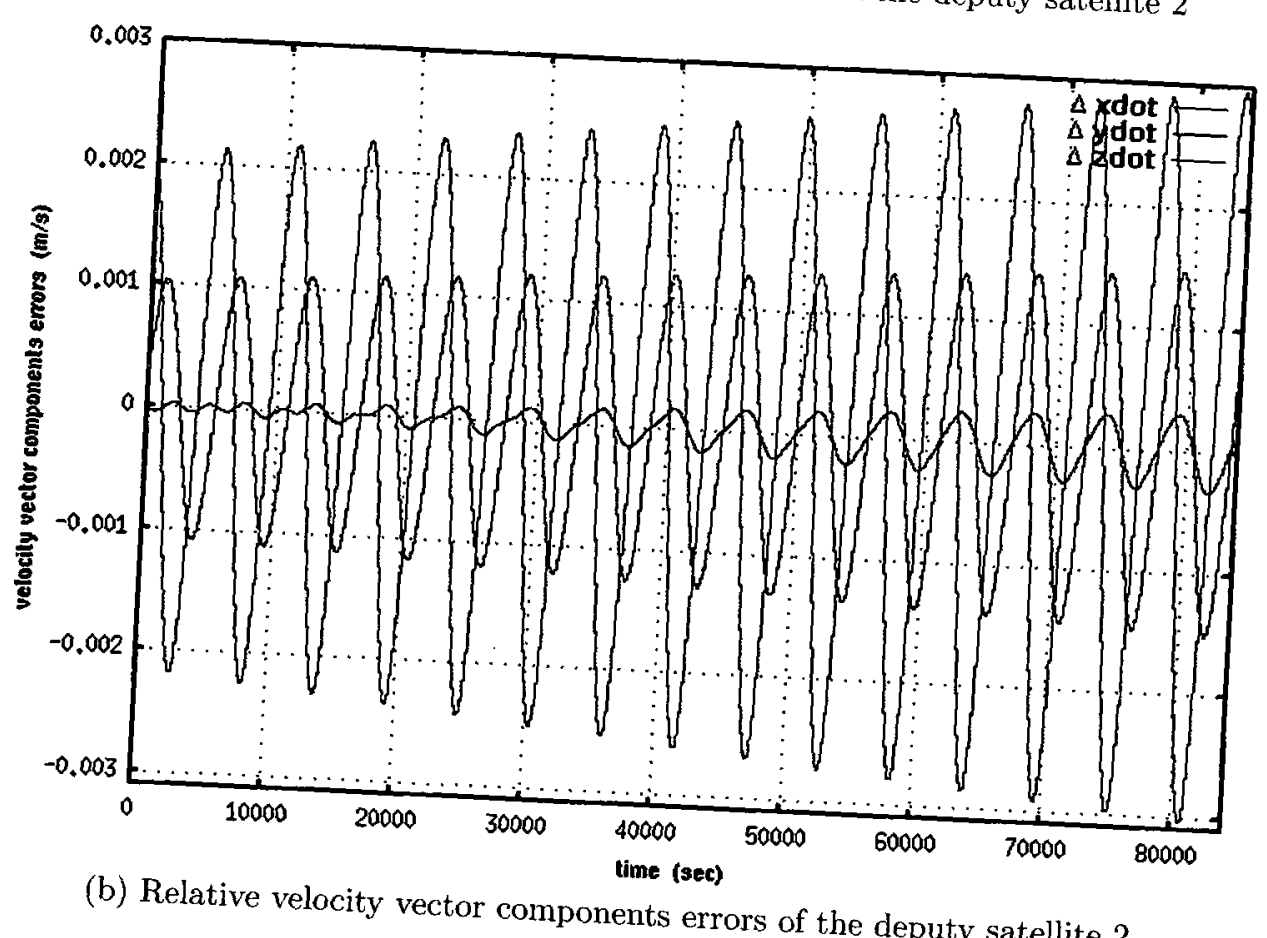

Figure 2.15: Illustration of the deputy satellite 2 relative position and 2 in the test case 2 


\subsubsection{Satellites Far Formation Flight Test Case 3}

The initial conditions for this test case are chosen such that the projection of the relative orbit of each deputy satellite in the chief local horizontal plane is quasi-elliptic with the chief satellite located close to the center. The semi-major and semi-minor axes lengths of these projected elliptic relative orbits will be less than $25 \mathrm{~km}$ and 600 meters respectively. The chief satellite will have an eccentricity of 0.2 which will generate a moderate in-track drift in the relative motions of deputy satellites, even if there are no perturbations. Table 2.5 and 2.6 describe the initial conditions for the chief and two deputy satellites in test case 3.

\begin{tabular}{|l|c|c|c|c|c|c|}
\hline \multirow{2}{*}{ Chief Satellite } & $a_{0}(\mathrm{~m})$ & $e_{0}$ & $i_{0}(\mathrm{deg})$ & $\Omega_{0}(\mathrm{deg})$ & $\omega_{0}(\mathrm{deg})$ & $M_{0}(\mathrm{deg})$ \\
\cline { 2 - 7 } & $8500 \mathrm{e} 3$ & 0.2 & 70 & 45 & 20 & 29.7144 \\
\hline \multirow{2}{*}{ Deputy Satellite 1} & $\delta a_{0}(\mathrm{~m})$ & $\delta e_{0}$ & $\delta i_{0}(\mathrm{deg})$ & $\delta \Omega_{0}(\mathrm{deg})$ & $\delta \omega_{0}(\mathrm{deg})$ & $\delta M_{0}(\mathrm{deg})$ \\
\cline { 2 - 7 } & -1.272 & $1.7 \mathrm{e}-5$ & $2.6 \mathrm{e}-3$ & $3.2 \mathrm{e}-3$ & -0.3473 & 0.3462 \\
\hline
\end{tabular}

Table 2.5: Test case 3 initial conditions for the chief and deputy satellite 1

\begin{tabular}{|l|c|c|c|c|c|c|}
\hline \multirow{2}{*}{ Chief Satellite } & $a_{0}(\mathrm{~m})$ & $e_{0}$ & $i_{0}(\mathrm{deg})$ & $\Omega_{0}(\mathrm{deg})$ & $\omega_{0}(\mathrm{deg})$ & $M_{0}(\mathrm{deg})$ \\
\cline { 2 - 7 } & $8500 \mathrm{e} 3$ & 0.2 & 70 & 45 & 20 & 159.8033 \\
\hline \multirow{2}{*}{ Deputy Satellite 2} & $\delta a_{0}(\mathrm{~m})$ & $\delta e_{0}$ & $\delta i_{0}(\mathrm{deg})$ & $\delta \Omega_{0}(\mathrm{deg})$ & $\delta \omega_{0}(\mathrm{deg})$ & $\delta M_{0}(\mathrm{deg})$ \\
\cline { 2 - 7 } & -0.839 & $1.2 \mathrm{e}-5$ & $-4.05 \mathrm{e}-3$ & 0 & 0.3822 & -0.3820 \\
\hline
\end{tabular}

Table 2.6: Test case 3 initial conditions for the chief and deputy satellite 2

We run the test case 3 simulation for a duration of 15 chief orbit periods which is equal to 116985 seconds or almost 32.49 hours. The orbit periods for the deputy satellites however, are slightly smaller because we set the initial semi-major axis difference $\delta a_{0}$ to -1.272 and -0.839 meters for the deputy satellites 1 and 2 respectively. Figure 2.16 displays the chief and deputy satellites inertial and relative orbit trajectories for the test case 3 . Figure 2.16 a shows that a chief and two deputy satellites fly in formation in elliptical orbits with perigee/apogee altitudes of almost 422/3822 kilometers and with next to 70 degrees inclination. Figure $2.16 \mathrm{~b}$ exhibits the created 
relative orbit trajectories for the two deputy satellites. The big purple circle in the middle of Figure 2.16a depicts Earth and it is not to scale. The small purple square in the middle of Figure $2.16 \mathrm{~b}$ represents the chief satellite. The maximum range distance between the chief satellite and deputy satellites 1 and 2 are almost 22 and 24 kilometers respectively. 


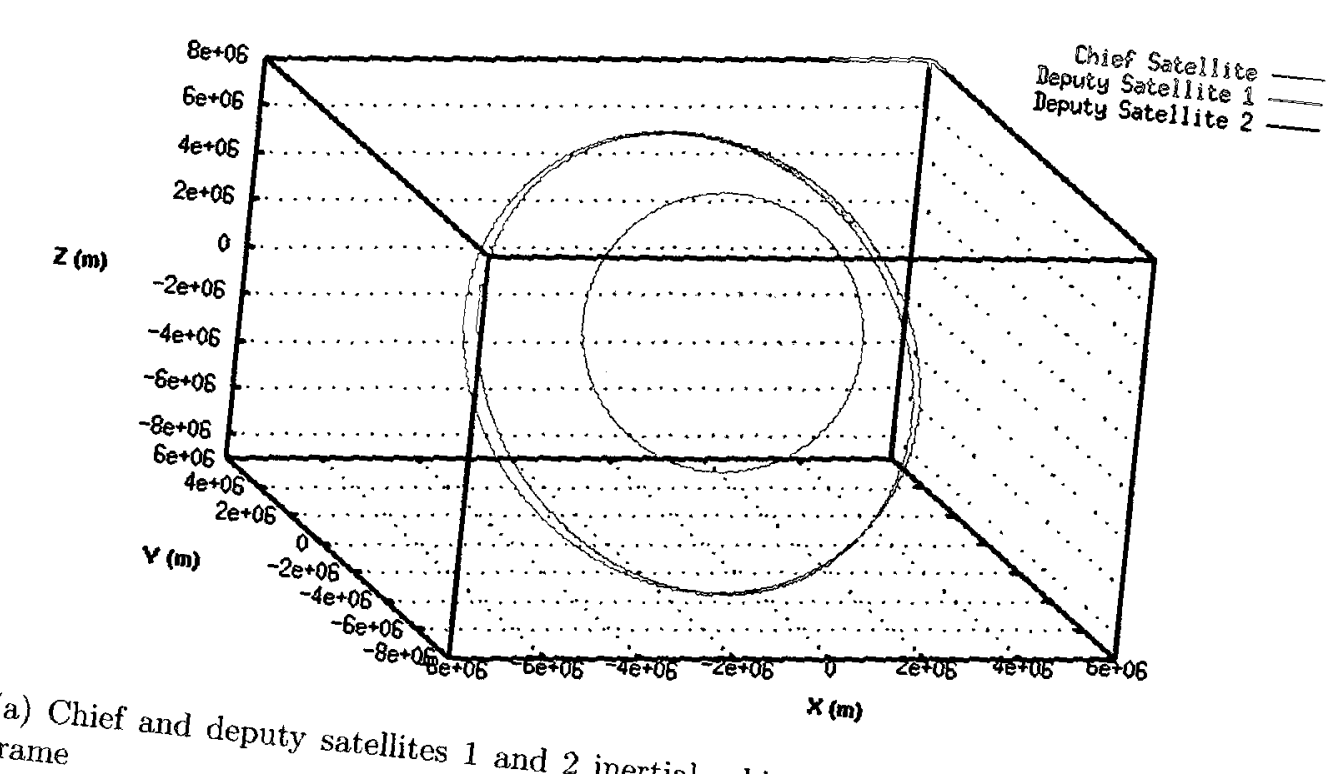

(a) Chief
frame

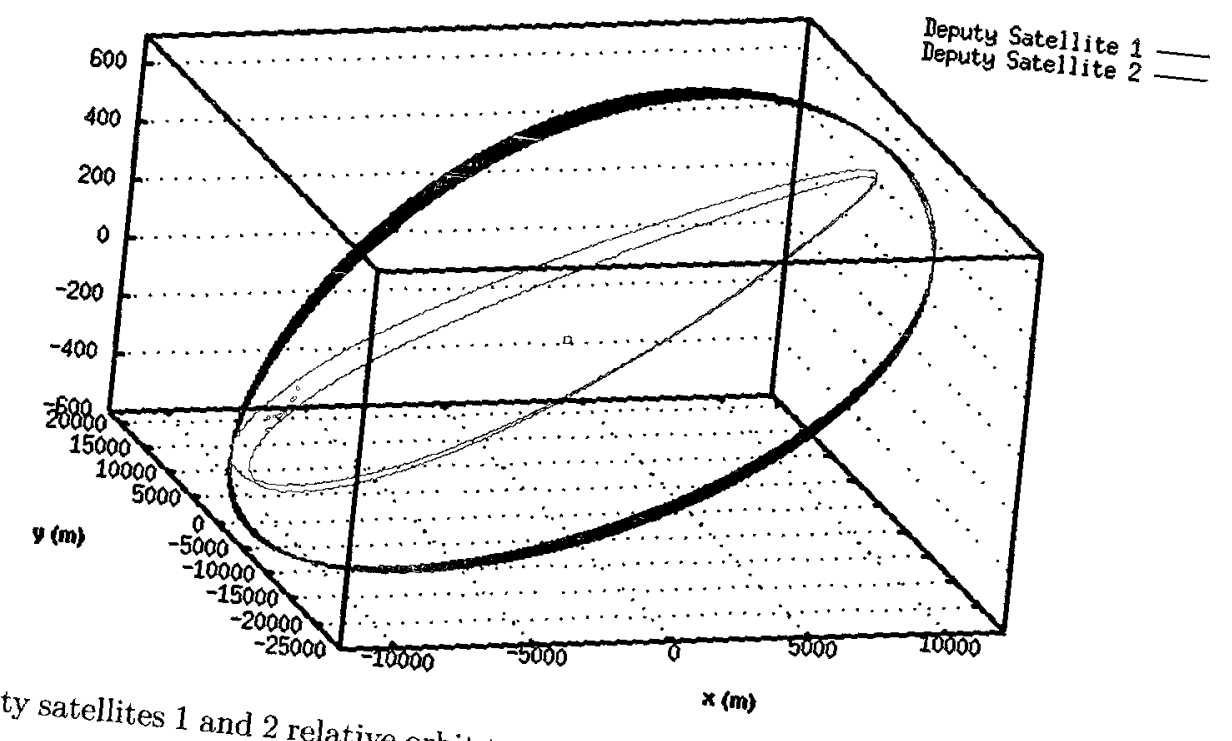

(b) Deputy satellites 1 and 2 relative orbit $\quad \times(m)$

Figure 2.16: Illustration of the chief and the chief LVLH rectilinear frame

trajectories in the test case 3 chief and deputy satellites' inertial and rectilinear frame 


\section{Simulation results for the deputy satellite 1}

Figure 2.17 displays the deputy satellite 1 actual relative position and velocity vector components in the chief LVLH curvilinear frame and compare it against the predicted relative position and velocity vectors components that are produced by the Gim-Alfriend close-form solution. Figure 2.18 demonstrates the performance of GimAlfriend analytical method in accomplishing the relative orbit prediction task for the deputy satellite 1. Figure $2.18 \mathrm{a}$ shows that the maximum absolute value of prediction errors for the deputy satellite 1 relative position vector components belongs to in-track element and is about 180 meters for the entire simulated flight times. The prediction error for the relative position vector radial element is significant as well. It reaches to 50 meters after 15 orbits. These large magnitudes for errors, however must have been expected because when we increase significantly the size of a relative orbit, the linearization of the deputy satellite position and velocity vectors about the chief satellite motion introduces correspondingly much larger errors into the GimAlfriend closed-form solution. Figure 2.18a also displays that there is a prominent secular growth in the prediction error for the in-track relative position vector component. The prediction errors for the radial and cross-track elements however, exhibit rather small secular changes. It must be clear to us that this conspicuous in-track secular growth has resulted partly from the neglected higher order terms of $J_{2}$ gravitational perturbation in the Gim-Alfriend state transition matrix. The ignored $J_{3}$ to $J_{6}$ gravitational perturbations have also contributed to this secular increase in error. Considering equations (2.147), we anticipate that the secular growth in prediction errors due to the discarded higher order terms of $J_{2}$ gravitational perturbation will be largely amplified if we even slightly add to the eccentricity. Turning our attention to Figure 2.18b, we note that the maximum absolute value of prediction errors for the deputy satellite 1 relative velocity vector components belongs to in-track element as well and it is almost 11 centimeters per second. Moreover there are indications of 
small secular changes in the prediction errors of radial and in-track relative velocity vector elements during the entire 32.49 hours of simulated flight times.

We infer from these test case 3 simulation results that the Gim-Alfriend closedform solution provides poor predictions for the deputy satellite 1 relative position and velocity vectors. This weak performance clearly is the direct consequence of having a large relative orbit size for the deputy satellite 1 . 

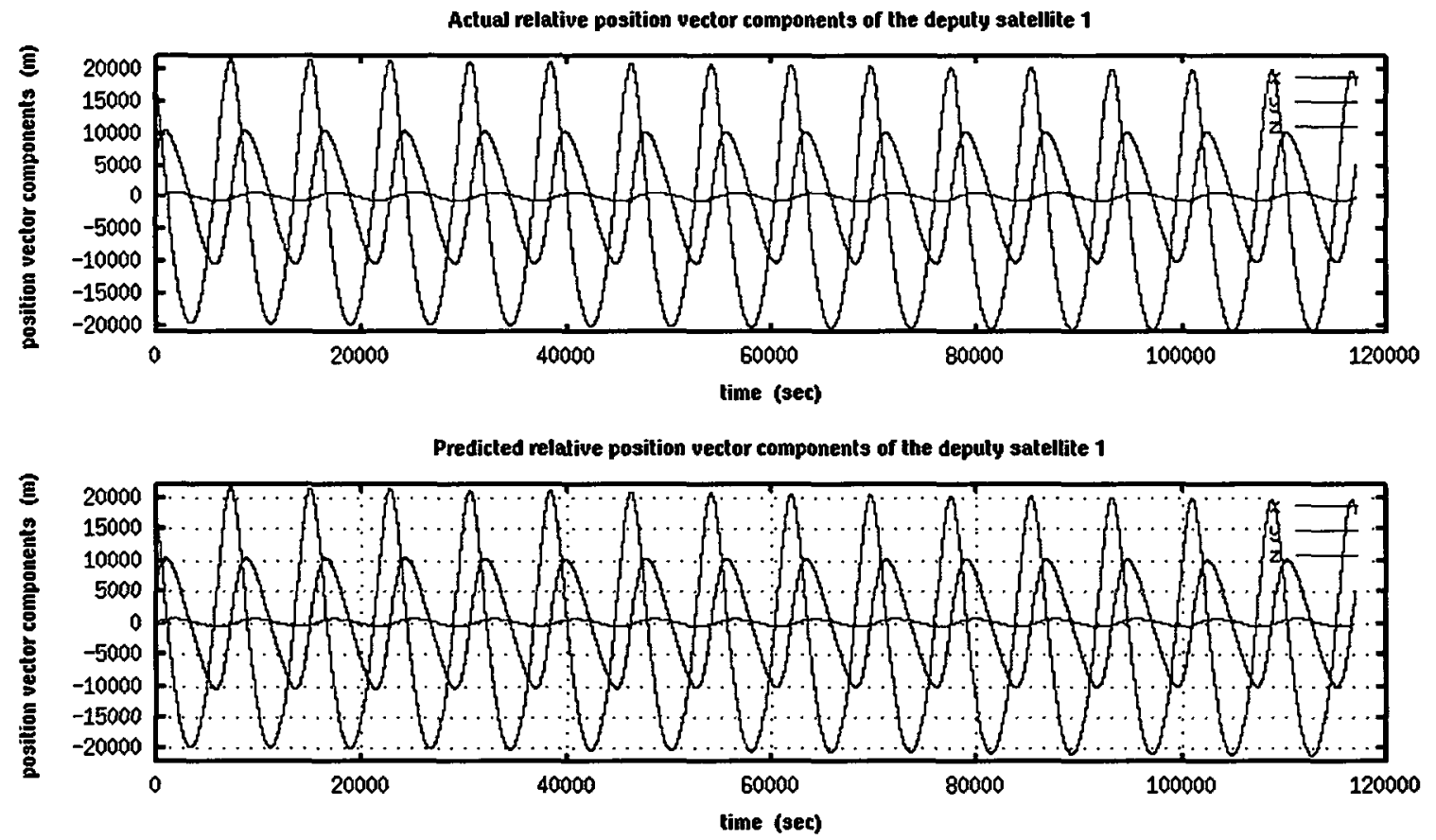

(a) Actual and predicted relative position vectors components of the deputy satellite 1
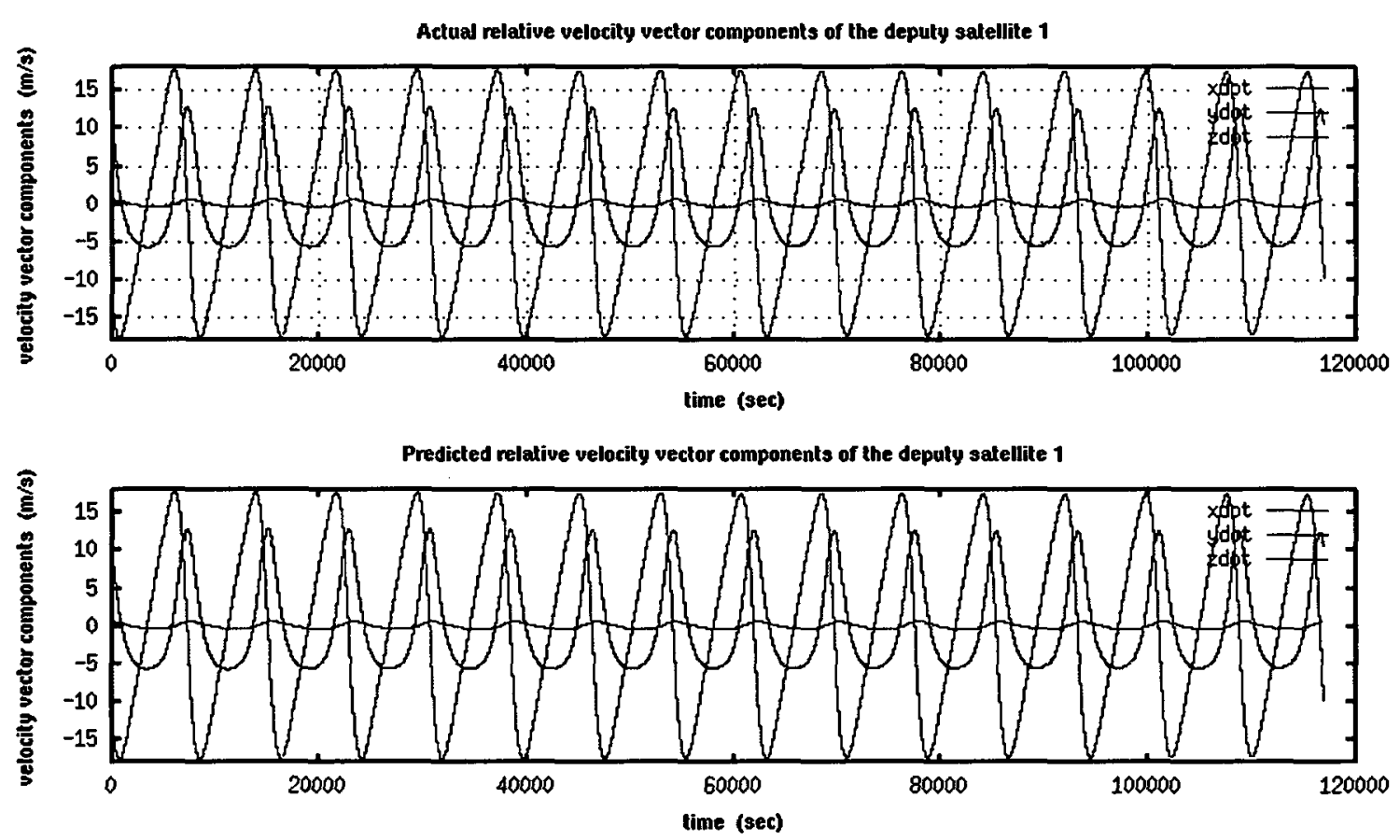

(b) Actual and predicted relative velocity vectors components of the deputy satellite 1

Figure 2.17: Illustration of the deputy satellite 1 actual/predicted relative position and velocity vectors components in the test case 3 


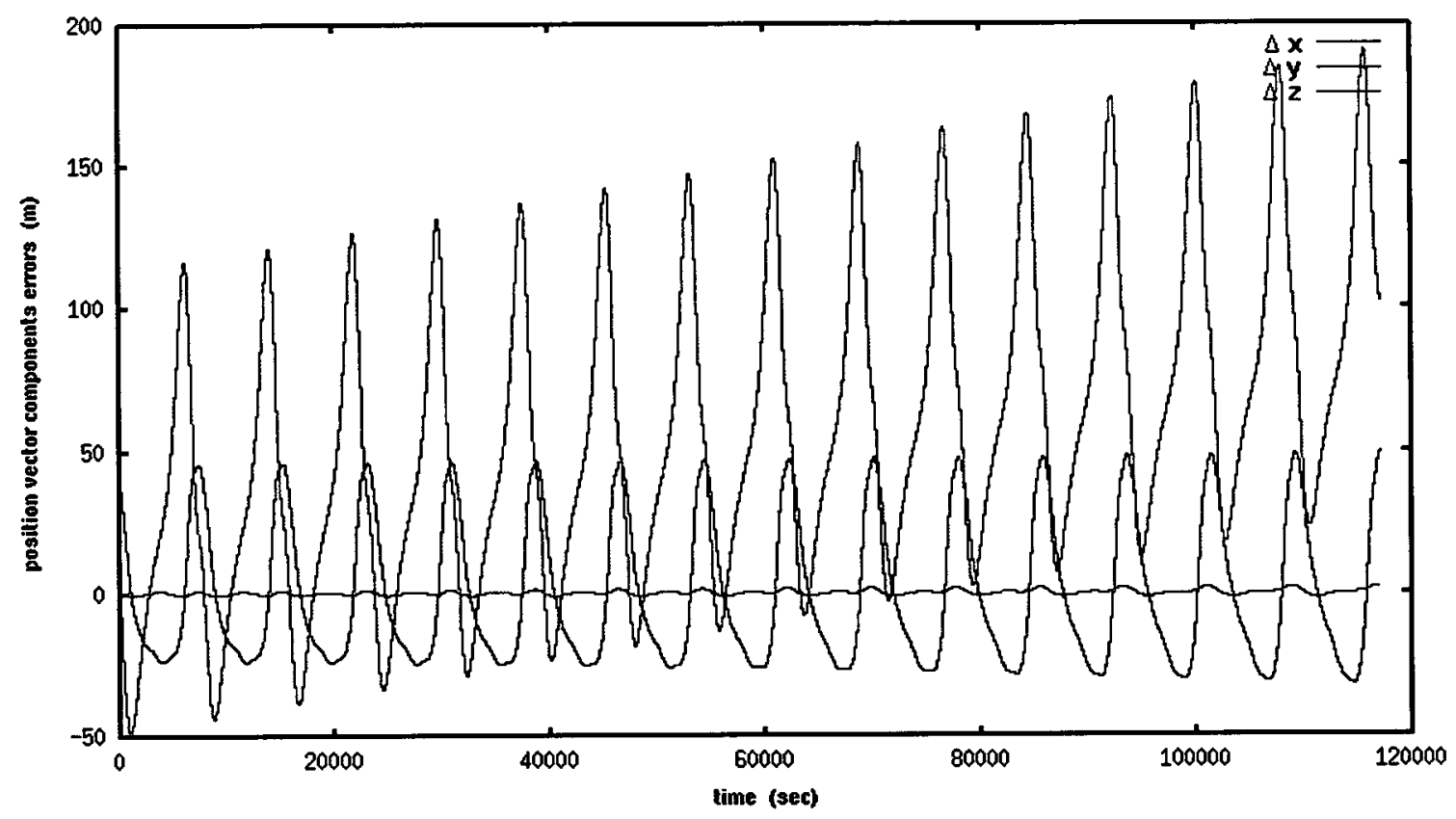

(a) Relative position vector components errors of the deputy satellite 1

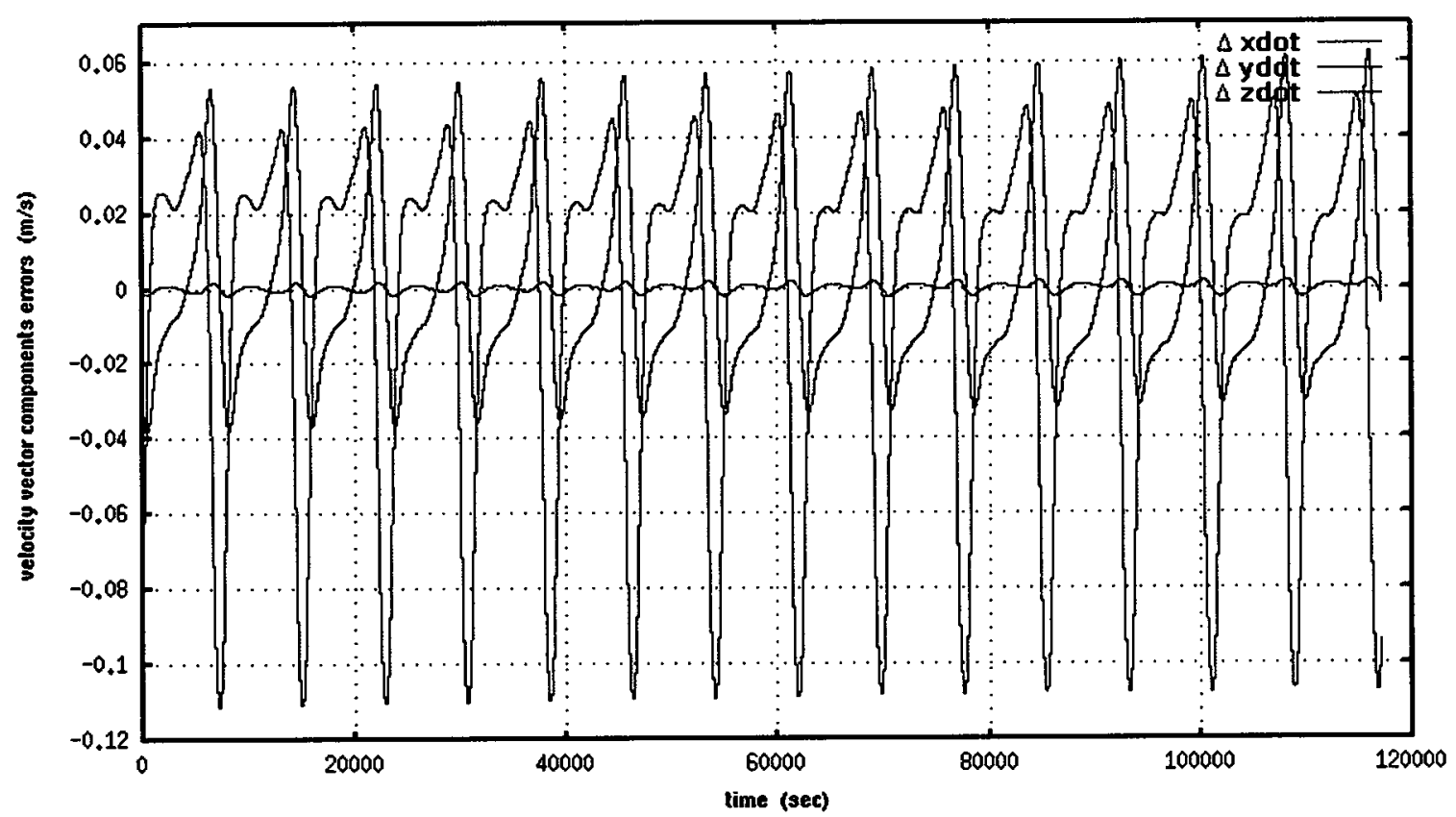

(b) Relative velocity vector components errors of the deputy satellite 1

Figure 2.18: Illustration of the deputy satellite 1 relative position and velocity errors in the test case 3 


\section{Simulation results for the deputy satellite 2}

Figure 2.19 displays the deputy satellite 2 actual relative position and velocity vector components in the chief LVLH curvilinear frame and compare it against the predicted relative position and velocity vectors components that are produced by the Gim-Alfriend close-form solution. Figure 2.20 demonstrates the performance of GimAlfriend analytical method in accomplishing the relative orbit prediction task for the deputy satellite 2. Figure 2.20a shows that the maximum absolute value of prediction errors for the deputy satellite 2 relative position vector components belongs to in-track element and is about 120 meters for the entire simulated flight times. The prediction error for the relative position vector radial element is significant as well. It is almost 65 meters during 15 orbits. These large magnitudes for errors, however are expected because when we increase significantly the size of a relative orbit, the linearization of the deputy satellite position and velocity vectors about the chief satellite motion introduces correspondingly much larger errors into the Gim-Alfriend closed-form solution. Figure 2.20a further displays that there are subtle secular changes in the prediction errors for the in-track and cross-track relative position vector components. The prediction error for the radial element however, exhibits no secular change. It should be clear that these slow in-track and cross-track secular growths have resulted partly from the neglected higher order terms of $J_{2}$ gravitational perturbation in the Gim-Alfriend state transition matrix. The ignored $J_{3}$ to $J_{6}$ gravitational perturbations have also contributed to these secular increases in errors. We should anticipate that the secular growth in prediction errors due to the discarded higher order terms of $J_{2}$ gravitational perturbation will be largely amplified if we even slightly add to the eccentricity. Turning our attention to Figure 2.20b, we note that the maximum absolute value of prediction errors for the deputy satellite 2 relative velocity vector components belongs to in-track element and it is almost 15 centimeters per second. Moreover there are no discernible secular changes in the prediction errors of all relative 
velocity vector elements during the entire 32.49 hours of simulated flight times.

We infer from these test case 3 simulation results that the Gim-Alfriend closedform solution provides poor predictions for the deputy satellite 2 relative position and velocity vectors. This weak performance clearly is the direct consequence of having a large relative orbit size for the deputy satellite 2 . 

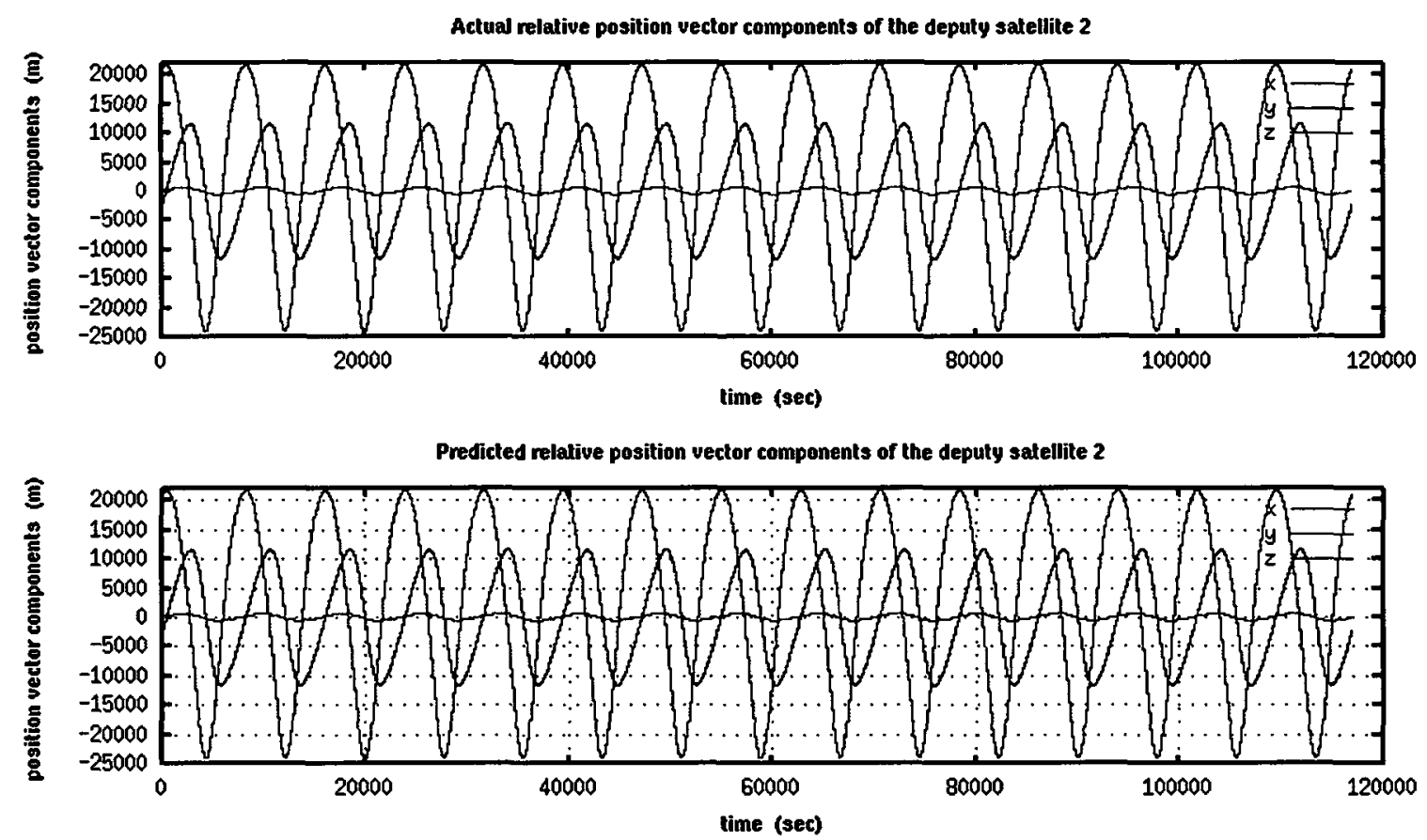

(a) Actual and predicted relative position vectors components of the deputy satellite 2
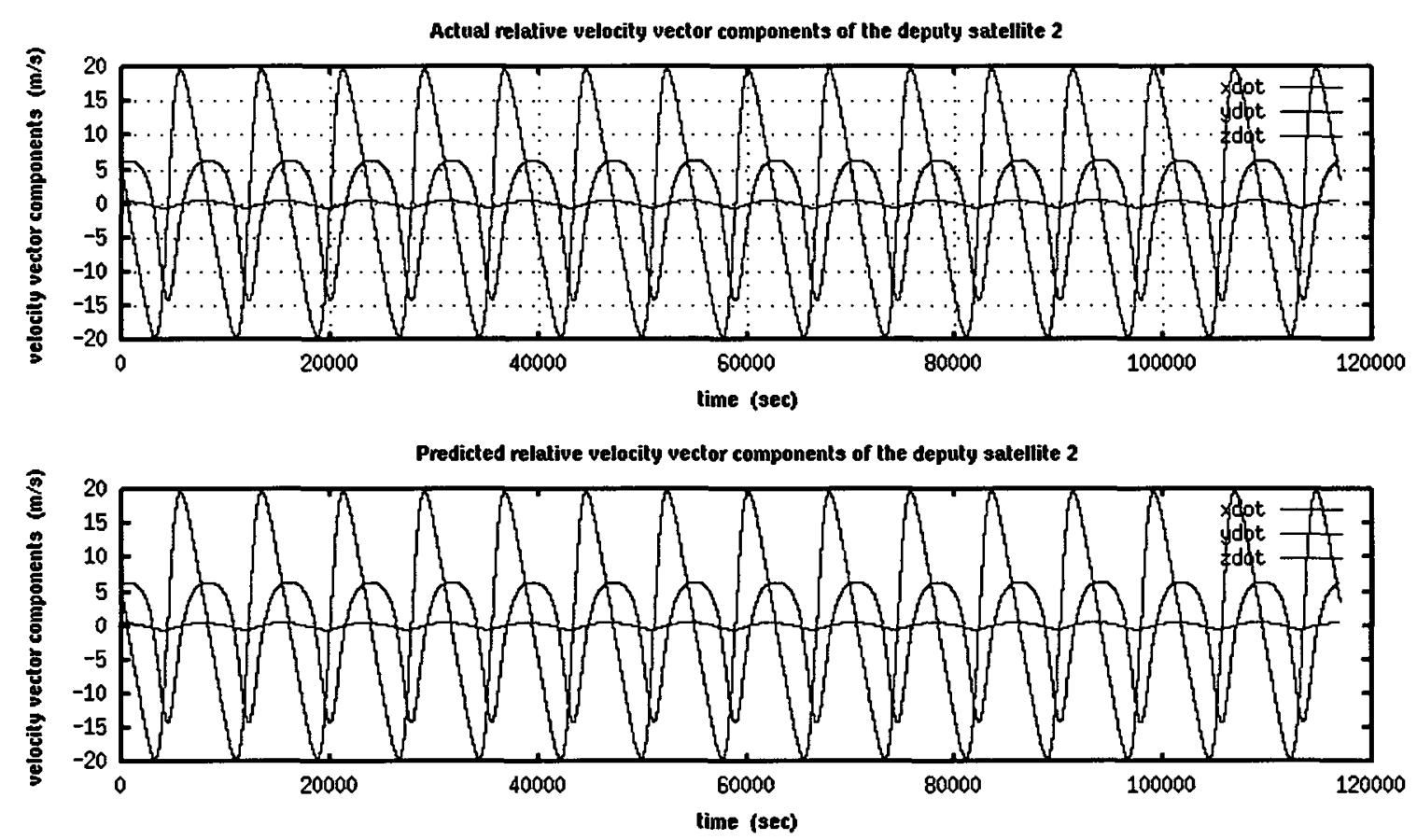

(b) Actual and predicted relative velocity vectors components of the deputy satellite 2

Figure 2.19: Illustration of the deputy satellite 2 actual/predicted relative position and velocity vectors components in the test case 3 


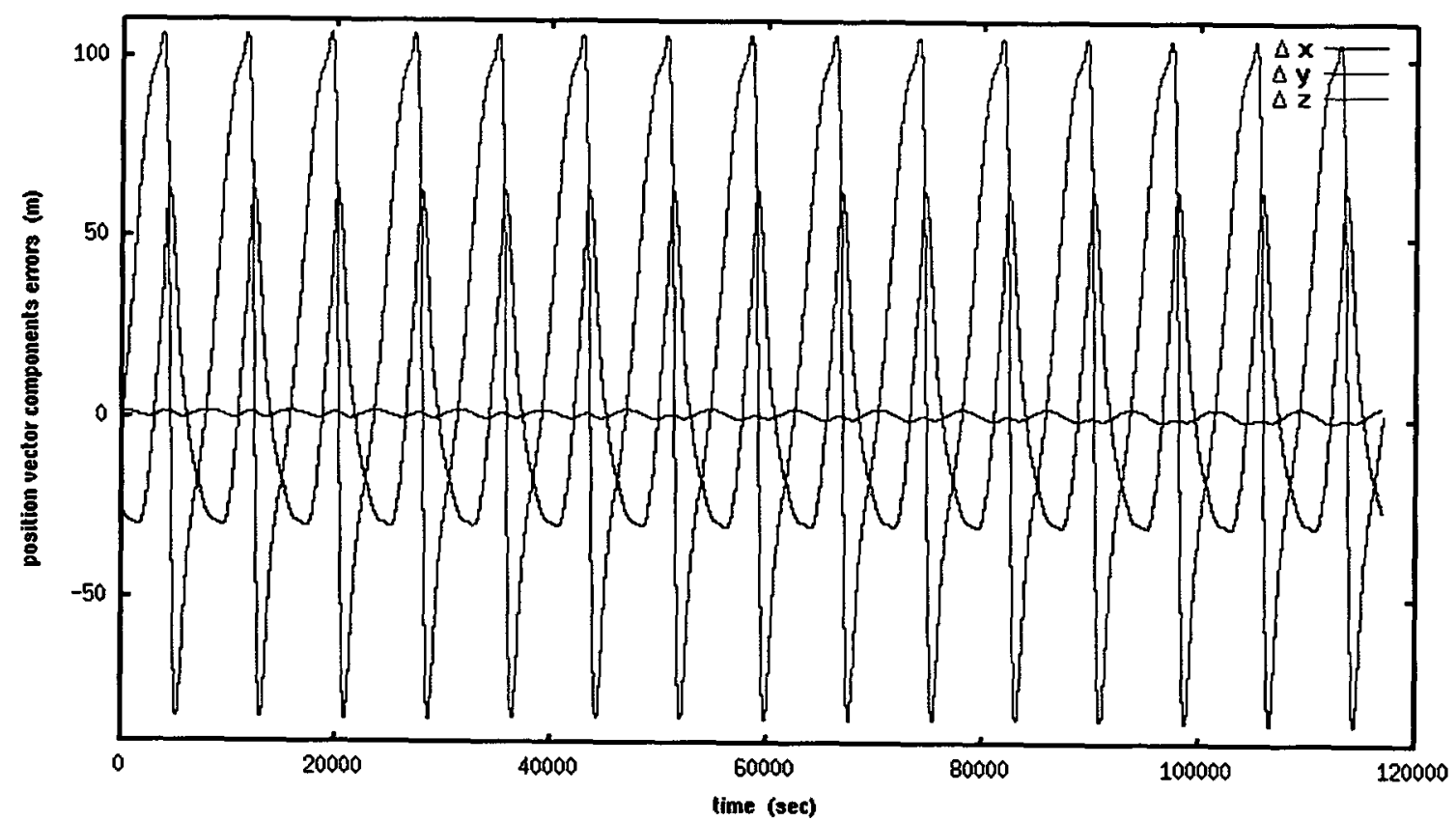

(a) Relative position vector components errors of the deputy satellite 2

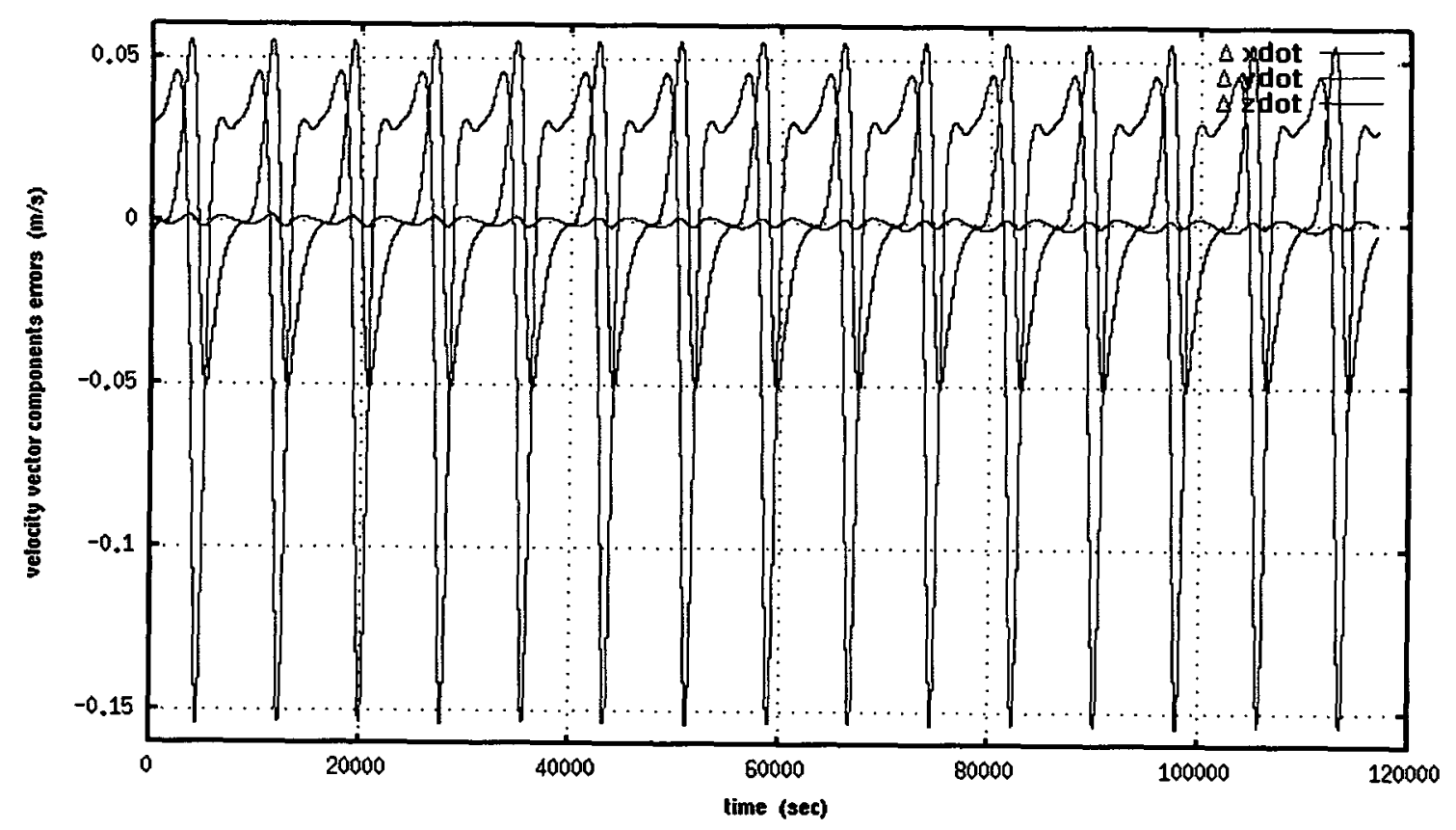

(b) Relative velocity vector components errors of the deputy satellite 2

Figure 2.20: Illustration of the deputy satellite 2 relative position and velocity errors in the test case 3 


\section{Chapter 3}

\section{Radar Observation and Relative Orbit State Estimation}

The primary purpose of the relative orbit determination is to provide an estimate of any deputy satellite relative state vector $\boldsymbol{X}$, using the Gim-Alfriend system dynamics linear model $\boldsymbol{X}=\Phi_{\boldsymbol{X}}\left(t, t_{0}\right) \boldsymbol{X}_{0}$, and some observation data $\boldsymbol{Z}$. We assume that the observation data $\boldsymbol{Z}$ is collected by a radar which is fixed on the chief satellite and it is often corrupted by noise. We further suppose that the radar observation data $\boldsymbol{Z}$ consists of range $\rho$, azimuth $a z$, elevation $e l$, and time of each observation $t_{k}$ for any given deputy satellite in a close formation flight. The geometry associated with the radar observation of one deputy satellite is shown in Figure 3.1. The range $\rho$ is the deputy satellite distance from the chief. The azimuth $a z$ is an angle on the chief local vertical plane measured clockwise from the nadir line to the location right beneath the deputy satellite. The elevation $e l$ is an angle measured from the chief local vertical plane to the deputy satellite with positive in the direction of the chief angular momentum vector. Azimuth az varies from $0^{\circ}$ to $360^{\circ}$ whereas elevation el takes on values from $-90^{\circ}$ to $90^{\circ}$. From Figure 3.1 , we can express the deputy satellite 


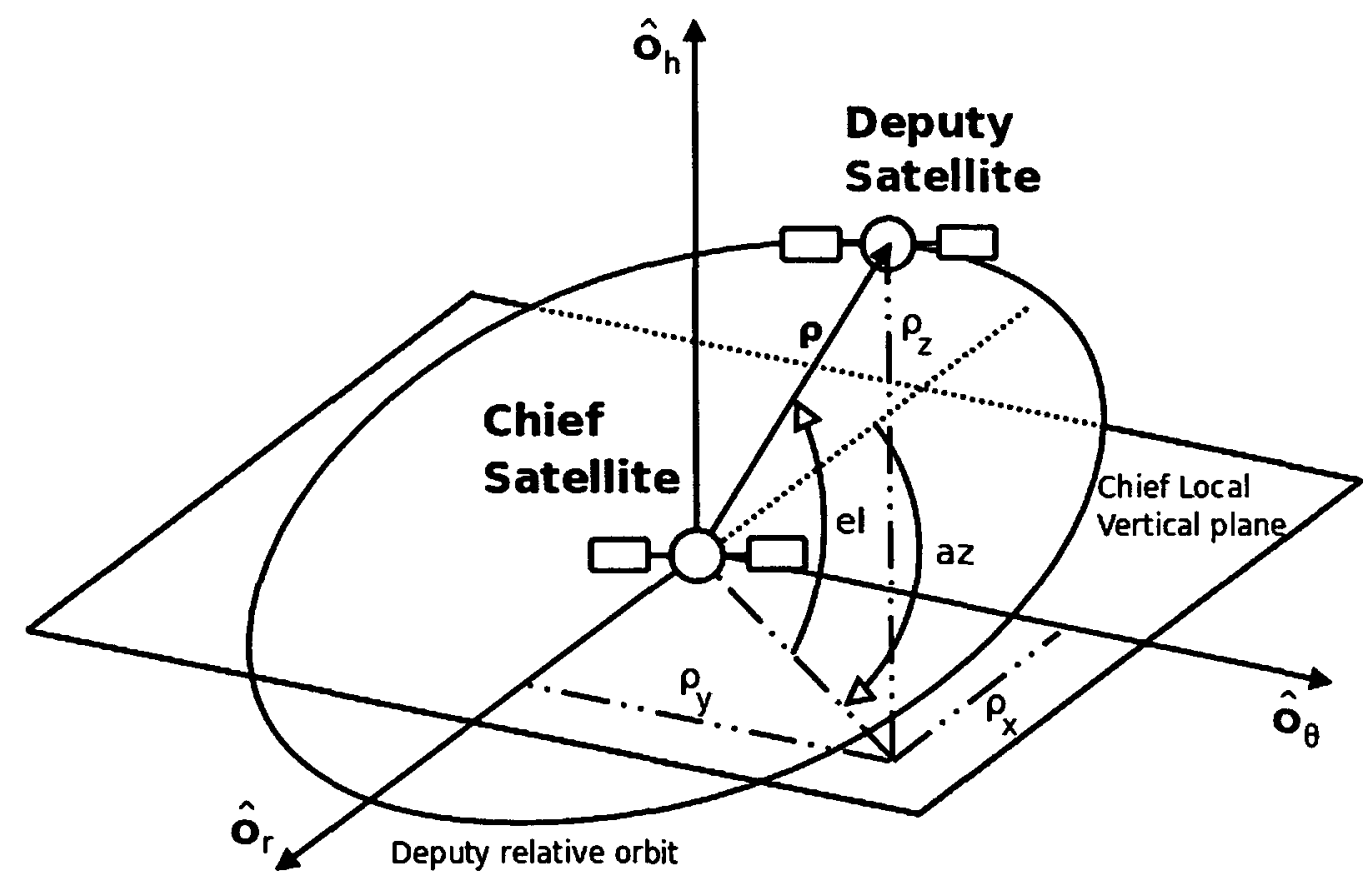

Figure 3.1: Geometry of the deputy satellite radar observation in the chief rectilinear LVLH frame

relative position vector $\boldsymbol{\rho}$ in the chief rectilinear $\mathrm{LVLH}$ frame by

$$
\boldsymbol{\rho}=\left[\begin{array}{c}
\rho_{x} \\
\rho_{y} \\
\rho_{z}
\end{array}\right]=\left[\begin{array}{c}
-\rho \cos (e l) \cos (a z) \\
\rho \cos (e l) \sin (a z) \\
\rho \sin (e l)
\end{array}\right]
$$

Since the radar observations will be used to update the estimate of the deputy satellite relative state vector $\boldsymbol{X}$, we must provide a mathematical model which relates the observations $\boldsymbol{Z}$ to the states $\boldsymbol{X}$, i.e.

$$
\boldsymbol{Z}=\boldsymbol{h}(\boldsymbol{X}(t), t)
$$


We can express the observations $\boldsymbol{Z}$ as functions of the slant range vector $\boldsymbol{\rho}$ by

$$
\begin{aligned}
\rho & =\sqrt{\rho_{x}^{2}+\rho_{y}^{2}+\rho_{z}^{2}} \\
a z & =\tan ^{-1}\left(\frac{\rho_{y}}{-\rho_{x}}\right) \\
e l & =\sin ^{-1}\left(\frac{\rho_{z}}{\sqrt{\rho_{x}^{2}+\rho_{y}^{2}+\rho_{z}^{2}}}\right)
\end{aligned}
$$

We also notice from Figure 2.5 that for close formation flights of spacecrafts, the angles $\phi$ and $\xi$ are very small. Thus the deputy satellite curvilinear in-track and cross-track distances from the chief satellite are approximately equal to $\rho_{y}$ and $\rho_{z}$ respectively. Hence we can write

$$
\begin{aligned}
& \rho \approx \sqrt{x^{2}+y^{2}+z^{2}} \\
& a z \approx \tan ^{-1}\left(\frac{y}{-x}\right) \\
& e l \approx \sin ^{-1}\left(\frac{z}{\sqrt{x^{2}+y^{2}+z^{2}}}\right)
\end{aligned}
$$

For the deputy satellite relative orbit determination problem, we utilize the GimAlfriend linear system model in discrete-time form with white Gaussian noise $\boldsymbol{W}(t)$ added. We also employ the nonlinear measurement model expressed by equation (3.2) in its discrete-time form and add white Gaussian noise $\boldsymbol{V}(t)$ to it.

Whiteness implies that the noise value is not correlated in time. Stated more simply, if we know the value of the noise at a given time, this knowledge does not help us in predicting what the noise value will be at any other time. Whiteness also implies that the noise has equal power at all frequencies within the system frequency bandwidth. One might argue that for the deputy satellite relative orbit determination problem, the process and measurement noise power are likely variable or each noise is in fact time correlated. In other word, the process and/or measurement noise is colored. For such cases, a white noise put through a small linear system can duplicate 
virtually any form of time-correlated noise. ${ }^{[28]}$ Therefore we can add a small linear system, called the "shaping filter" to our original system and achieve an overall linear system driven by white noise once again. In this research, however, we will only treat the white noise models to make the mathematics involved more tractable.

Whereas whiteness pertains to time or frequency relationships of a noise, Gaussianness has to do with noise amplitude. The probability density of a Gaussian noise amplitude takes on the form of a normal bell-shaped curve at any single point in time. Our assumption on gaussianness is physically justified by the fact that a process or measurement noise often caused by a number of small sources. It has been mathematically proven that when a number of independent random variables are added together, the summed effect can be described very closely by a Gaussian probability density function regardless of the shape of the individual densities. There is also a practical justification for using Gaussian densities. We know at best the first and second order statistics (mean and variance) of a noise process and in the absence of any higher order statistics, there is no better form to assume than the Gaussian density. Note that the first and second order statistics can completely define a Gaussian density, unlike most other densities which require an endless number of orders of statistics to specify their shape entirely.

We now express the system truth model stochastic equations for the deputy satellite relative orbit determination problem by

$$
\begin{aligned}
\boldsymbol{X}_{k+1} & =\Phi_{\boldsymbol{X}}\left(t_{k+1}, t_{k}\right) \cdot \boldsymbol{X}_{k}+\boldsymbol{W}_{k} \\
\boldsymbol{Z}_{k} & =\boldsymbol{h}\left(\boldsymbol{X}_{k}\right)+\boldsymbol{V}_{k}
\end{aligned}
$$

where $\boldsymbol{W}_{k}$ and $\boldsymbol{V}_{k}$ are zero-mean white Gaussian noise processes with their covari- 
ances given as follows

$$
\begin{aligned}
& E\left\{\boldsymbol{W}_{k} \cdot \boldsymbol{W}_{j}^{T}\right\}= \begin{cases}0 & k \neq j \\
Q & k=j\end{cases} \\
& E\left\{\boldsymbol{V}_{k} \cdot \boldsymbol{V}_{j}^{T}\right\}= \begin{cases}0 & k \neq j \\
R & k=j\end{cases}
\end{aligned}
$$

$Q$ is a 6-by-6 system noise covariance matrix and $R$ is a 3 -by-3 measurement noise covariance matrix. We further assume that $\boldsymbol{W}_{k}$ and $\boldsymbol{V}_{k}$ are uncorrelated so that $E\left\{\boldsymbol{V}_{k} \cdot \boldsymbol{W}_{k}^{T}\right\}=0$ for all $k$. Note that the system noise covariance matrix $Q$ serves as a measure of uncertainty in the system dynamics model. Unmodeled forces due to ignored zonal and tesseral geopotential harmonics, atmospheric drag and third body perturbation constitute the major sources of error for the Gim-Alfriend relative motion system dynamics model. The measurement noise covariance matrix $R$ also quantifies the accuracy of the radar observations.

\subsection{Extended Kalman Filter}

In this research we utilize a Kalman filter to determine the deputy satellites relative state estimates based on all of the measurement data, up to and including the most recent radar observation. We know that the kalman filter is an optimal sequential estimator for a linear problem subject to zero-mean white Gausssian noise [28]. For a nonlinear problem, however, the Kalman filter often doesn't provide the optimal estimate of the system states. Fortunately, the Kalman filter has been expanded to include nonlinearity in dynamic and measurement models, which led to the development of the extended Kalman filter. The extended Kalman filter has been successfully applied to many nonlinear problems as some described by Maybeck [29], Gelb ${ }^{[30]}$, and Jazwinski ${ }^{[31]}$. Because in the deputy satellite relative orbit deter- 
mination problem, the measurement model is nonlinear, we employ a discrete-time extended Kalman filter to estimate the relative states. The fundamental concept of the extended Kalman filter involves the notion that the true state is sufficiently close to the estimated state. Therefore, the system and measurement dynamics can be represented fairly accurately by a linearized first-order Taylor series expansion about some nominal state $\overline{\boldsymbol{X}}_{k}$. In the extended Kalman filter, the current state estimate $\hat{\boldsymbol{X}}_{k}$ is used for the nominal state, so that $\overline{\boldsymbol{X}}_{k}=\hat{\boldsymbol{X}}_{k}$. We write the first-order expansion of the nonlinear vector function $\boldsymbol{h}$ about the nominal state $\hat{\boldsymbol{X}}_{k}$ as follows

$$
\boldsymbol{h}\left(\boldsymbol{X}_{k}\right)=\boldsymbol{h}\left(\hat{\boldsymbol{X}}_{k}\right)+\left.\frac{\partial \boldsymbol{h}}{\partial \boldsymbol{X}}\right|_{\boldsymbol{X}=\hat{\boldsymbol{X}}_{k}} \cdot\left[\boldsymbol{X}_{k}-\hat{\boldsymbol{X}}_{k}\right]+\text { higher order terms }
$$

Ignoring higher order terms we have

$$
\boldsymbol{h}\left(\boldsymbol{X}_{k}\right) \approx \boldsymbol{Z}_{c, k}+H_{k}\left(\hat{\boldsymbol{X}}_{k}\right) \cdot \Delta \boldsymbol{X}_{k}
$$

where $\boldsymbol{Z}_{c, k}$ are the predicted values of the observations. The $H_{k}$ matrix is a 3 -by- 6 matrix that relates changes in the slant range $\rho$, azimuth $a z$, and elevation $e l$ to changes in the relative state $\boldsymbol{X}$ at the time of the observation $t_{k}$. Using equation (3.4), the $H_{k}$ matrix is found analytically by

$$
\begin{aligned}
H_{k}\left(\hat{\boldsymbol{X}}_{k}\right) \approx & {\left[\begin{array}{llllll}
\frac{\partial \rho}{\partial x} & \frac{\partial \rho}{\partial \dot{x}} & \frac{\partial \rho}{\partial y} & \frac{\partial \rho}{\partial \dot{y}} & \frac{\partial \rho}{\partial z} & \frac{\partial \rho}{\partial \dot{z}} \\
\frac{\partial a z}{\partial x} & \frac{\partial a z}{\partial \dot{x}} & \frac{\partial a z}{\partial y} & \frac{\partial a z}{\partial \dot{y}} & \frac{\partial a z}{\partial z} & \frac{\partial a z}{\partial \dot{z}} \\
\frac{\partial e l}{\partial x} & \frac{\partial e l}{\partial \dot{x}} & \frac{\partial e l}{\partial y} & \frac{\partial e l}{\partial \dot{y}} & \frac{\partial e l}{\partial z} & \frac{\partial e l}{\partial \dot{z}}
\end{array}\right]_{\boldsymbol{X}=\hat{\boldsymbol{X}}_{k}} } \\
\approx & {\left[\begin{array}{ccccccc}
-\cos \left(\hat{a z_{k}}\right) \cdot \cos \left(\hat{e} l_{k}\right) & 0 & \sin \left(\hat{a z_{k}}\right) \cdot \cos \left(\hat{e} l_{k}\right) & 0 & \sin \left(\hat{e} l_{k}\right) & 0 \\
\frac{\sin \left(\hat{a} z_{k}\right)}{\hat{\rho}_{k} \cdot \cos \left(\hat{e} l_{k}\right)} & 0 & \frac{\cos \left(\hat{a z_{k}}\right)}{\hat{\rho}_{k} \cdot \cos \left(\hat{e} l_{k}\right)} & 0 & 0 & 0 \\
\frac{\cos \left(\hat{a z_{k}}\right) \cdot \sin \left(\hat{e} l_{k}\right)}{\hat{\rho}_{k}} & 0 & \frac{-\sin \left(\hat{a z_{k}}\right) \cdot \sin \left(\hat{e} l_{k}\right)}{\hat{\rho}_{k}} & 0 & \frac{\cos \left(\hat{e} l_{k}\right)}{\hat{\rho}_{k}} & 0
\end{array}\right] }
\end{aligned}
$$


where

$$
\begin{aligned}
\hat{\rho}_{k} & \approx \sqrt{\hat{x}_{k}^{2}+\hat{y}_{k}^{2}+\hat{z}_{k}^{2}} \\
\hat{a z} z_{k} & \approx \tan ^{-1}\left(\frac{\hat{y}_{k}}{-\hat{x}_{k}}\right) \\
\hat{e l} & \approx \sin ^{-1}\left(\frac{\hat{z}_{k}}{\sqrt{\hat{x}_{k}^{2}+\hat{y}_{k}^{2}+\hat{z}_{k}^{2}}}\right)
\end{aligned}
$$

A derivation for the extended Kalman filter is presented by Maybeck ${ }^{29}$ ] and therefore will not be repeated here. The resulting discrete-time extended Kalman filter equations are :

Propagation equations

$$
\begin{aligned}
\hat{\boldsymbol{X}}_{k+1}^{-} & =\Phi_{\boldsymbol{X}}\left(t_{k+1}, t_{k}\right) \cdot \hat{\boldsymbol{X}}_{k}^{+} \\
P_{k+1}^{-} & =\Phi_{\boldsymbol{X}}\left(t_{k+1}, t_{k}\right) \cdot P_{k}^{+} \cdot \Phi_{\boldsymbol{X}}^{T}\left(t_{k+1}, t_{k}\right)+Q
\end{aligned}
$$

Update equations

$$
\begin{aligned}
& K_{k}=P_{k}^{-} \cdot H_{k}^{T}\left(\hat{\boldsymbol{X}}_{k}^{-}\right) \cdot\left\{H_{k}\left(\hat{\boldsymbol{X}}_{k}^{-}\right) \cdot P_{k}^{-} \cdot H_{k}^{T}\left(\hat{\boldsymbol{X}}_{k}^{-}\right)+R\right\}^{-1} \\
& \hat{\boldsymbol{X}}_{k}^{+}=\hat{\boldsymbol{X}}_{k}^{-}+K_{k} \cdot\left\{\boldsymbol{Z}_{k}-\boldsymbol{h}\left(\hat{\boldsymbol{X}}_{k}^{-}\right)\right\} \\
& P_{k}^{+}=P_{k}^{-}-K_{k} \cdot H_{k}\left(\hat{\boldsymbol{X}}_{k}^{-}\right) \cdot P_{k}^{-}
\end{aligned}
$$

$\hat{\boldsymbol{X}}_{k}^{-}$and $\hat{\boldsymbol{X}}_{k}^{+}$denote respectively a priori and a posteriori state estimates at time step k. $P_{k}^{-}$and $P_{k}^{+}$designate a priori and a posteriori 6-by-6 estimation error covariance matrices for the time $t_{k}$ as well. They are defined by

$$
\begin{aligned}
& P_{k}^{-}=E\left\{\left[\boldsymbol{X}_{k}-\hat{\boldsymbol{X}}_{k}^{-}\right] \cdot\left[\boldsymbol{X}_{k}-\hat{\boldsymbol{X}}_{k}^{-}\right]^{T}\right\} \\
& P_{k}^{+}=E\left\{\left[\boldsymbol{X}_{k}-\hat{\boldsymbol{X}}_{k}^{+}\right] \cdot\left[\boldsymbol{X}_{k}-\hat{\boldsymbol{X}}_{k}^{+}\right]^{T}\right\}
\end{aligned}
$$


$K_{k}$ represents a 6 -by-3 extended Kalman filter gain matrix and $Z_{k}$ indicates an observation column vector generated by the chief satellite radar. $\boldsymbol{h}\left(\hat{\boldsymbol{X}}_{k}^{-}\right)$yields the predicted values for the observation using a priori state estimate. The propagation equations (3.11) and (3.12) project forward in time separately the current state estimate and the state estimation error covariance to obtain the corresponding a priori estimates for the next time step. The update equations (3.13), (3.14), and (3.15) first compute the extended Kalman filter gain, then incorporate a new measurement into the a priori state estimates to provide an improved a posteriori estimate, and at the end update the state estimation error covariance. The initial values for the state estimate column vector and the estimation error covariance matrix must be input to the extended kalman filter. Thus we must have

$$
\begin{aligned}
& \hat{\boldsymbol{X}}_{0}^{+}=\hat{\boldsymbol{X}}_{0} \\
& P_{0}^{+}=E\left\{\left[\boldsymbol{X}_{0}-\hat{\boldsymbol{X}}_{0}\right] \cdot\left[\boldsymbol{X}_{0}-\hat{\boldsymbol{X}}_{0}\right]^{T}\right\}
\end{aligned}
$$

For the deputy satellite relative orbit determination problem, we employ equation (2.53) and the orbit elements of the chief and deputy satellites at time $t_{0}$ to find the initial relative state estimate $\hat{\boldsymbol{X}}_{0}$. The initial estimation error covariance matrix $P_{0}^{+}$ represents a measure of confidence in the initial values of the relative state estimate $\hat{\boldsymbol{X}}_{0}$

We now specify a few rather nonrestrictive conditions under which the discretetime extended Kalman filter algorithm is stable. The extended Kalman filter stability is proved using Lyapunov's direct method ${ }^{[32]}$. We must show that the estimation error dynamics $\tilde{\boldsymbol{X}}_{k} \equiv \boldsymbol{X}_{k}-\hat{\boldsymbol{X}}_{k}$, are stable. A candidate Lyapunov function is as follows

$$
V(\tilde{\boldsymbol{X}})=\tilde{\boldsymbol{X}}_{k}^{T} \cdot P_{k}^{-1} \cdot \tilde{\boldsymbol{X}}_{k}
$$

If the state estimation error covariance matrix $P_{k}$ is positive definite at any time step 
$k$, then $V(\tilde{\boldsymbol{X}})>0$ for all $\tilde{\boldsymbol{X}}_{k} \neq 0$. We can apply Lyapunov's theorem of asymptotic stability, if we show that $\Delta V(\tilde{\boldsymbol{X}})<0$. The increment of $V(\tilde{\boldsymbol{X}})$ is given by

$$
\Delta V(\tilde{\boldsymbol{X}})=\tilde{\boldsymbol{X}}_{k+1}^{T} \cdot P_{k+1}^{-1} \cdot \tilde{\boldsymbol{X}}_{k+1}-\tilde{\boldsymbol{X}}_{k}^{T} \cdot P_{k}^{-1} \cdot \tilde{\boldsymbol{X}}_{k}
$$

Crassidis and Junkins ${ }^{[32]}$ revealed that the increment of $V(\tilde{\boldsymbol{X}})$ is negative definite if the following inequality is satisfied.

$$
-\left[\Phi_{\boldsymbol{X}}\left(t_{k+1}, t_{k}\right) \cdot K_{k} \cdot R \cdot K_{k}^{T} \cdot \Phi_{\boldsymbol{X}}^{T}\left(t_{k+1}, t_{k}\right)+Q\right]<0
$$

It is clear that if $R$ matrix is positive definite and $Q$ matrix is at least positive semidefinite then the Lyapunov condition for asymptotic stability is satisfied and the discrete-time extended Kalman filter is stable. We should however, recall that the function $V(\tilde{\boldsymbol{X}})$ is a Lyapunov function at the first place if matrix $P_{k}$ is a positive definite matrix at each time step $k$. Crassidis and Junkins addressed this issue as well and proved that if $R$ and $P_{k}$ matrices are positive definite, then matrix $P_{k+1}$ is also positive definite. Therefore we can have a stable extended Kalman filter if we make matrices $R$ and $P_{0}$ positive definite and make matrix $Q$ at least positive semidefinite. In this research, we assume $Q, R$, and $P_{0}$ matrices are all diagonal matrices and all their diagonal elements are positive too. Therefore $Q, R$, and $P_{0}$ matrices are all positive definite. We express the diagonal 6-by-6 initial state estimation error covariance matrix $P_{0}$ as follows

$$
P_{0}^{+}=\operatorname{diag}\left[\left(x_{0}-\hat{x}_{0}\right)^{2},\left(\dot{x}_{0}-\hat{\dot{x}}_{0}\right)^{2},\left(y_{0}-\hat{y}_{0}\right)^{2},\left(\dot{y}_{0}-\hat{\dot{y}}_{0}\right)^{2},\left(z_{0}-\hat{z}_{0}\right)^{2},\left(\dot{z}_{0}-\hat{\dot{z}}_{0}\right)^{2}\right]
$$

Similarly for the chief satellite radar, $R$ will be a 3-by-3 diagonal matrix where its diagonal elements are equal to the variances in the slant range, azimuth, and elevation 
measurements respectively. i.e.

$$
R=\left[\begin{array}{ccc}
\sigma_{\rho}^{2} & 0 & 0 \\
0 & \sigma_{a z}^{2} & 0 \\
0 & 0 & \sigma_{e l}^{2}
\end{array}\right]
$$

Note that symbol $\sigma$ denotes the standard deviation.

\subsection{Iterated Extended Kalman Filter}

Several modifications to the extended Kalman filter can be incorporated to improve the filter performance. One change is the inclusion of "pseudonoise" [29] in the $Q$ and $R$ matrices to compensate for unmodeled dynamics and/or errors due to neglected nonlinearities in the problem. This method is used to alter the Kalman filter gain and achieve a balance between ignoring the new observation data (low filter gain) and tracking the noise in the measurements (high filter gain). Another modification to compensate for the neglected nonlinearities in the problem is to add iterations to the propagation and update equations. In this research, we investigate an iterated extended Kalman filter (IEKF) which embodies local iterations only in the update equations. The modified update equations are given by ${ }^{[32]}$ :

IEKF update equations

$$
\begin{aligned}
& K_{k_{i}}=P_{k}^{-} \cdot H_{k}^{T}\left(\hat{\boldsymbol{X}}_{k_{i}}^{+}\right) \cdot\left\{H_{k}\left(\hat{\boldsymbol{X}}_{k_{i}}^{+}\right) \cdot P_{k}^{-} \cdot H_{k}^{T}\left(\hat{\boldsymbol{X}}_{k_{i}}^{+}\right)+R\right\}^{-1} \\
& \hat{\boldsymbol{X}}_{k_{i}}^{+}=\hat{\boldsymbol{X}}_{k}^{-}+K_{k_{i}} \cdot\left\{\boldsymbol{Z}_{k}-\boldsymbol{h}\left(\hat{\boldsymbol{X}}_{k_{i}}^{+}\right)-H_{k}\left(\hat{\boldsymbol{X}}_{k_{i}}^{+}\right) \cdot\left[\hat{\boldsymbol{X}}_{k}^{-}-\hat{\boldsymbol{X}}_{k_{i}}^{+}\right]\right\} \\
& P_{k_{i}}^{+}=P_{k}^{-}-K_{k_{i}} \cdot H_{k}\left(\hat{\boldsymbol{X}}_{k_{i}}^{+}\right) \cdot P_{k}^{-}
\end{aligned}
$$


with $\hat{\boldsymbol{X}}_{k_{0}}^{+}=\hat{\boldsymbol{X}}_{k}^{-}$. At each time step $k$, equations (3.24), (3.25), and (3.26) are repeatedly calculated until the solution converges or a maximum number of iterations is reached. The algorithm for our iterated extended Kalman filter is as follows :

1. Set $k=0$.

2. Read in the initial state estimate vector $\hat{\boldsymbol{X}}_{0}^{+}$, and the initial estimation error covariance matrix $P_{0}^{+}$.

3. Propagate the current state estimate vector $\hat{\boldsymbol{X}}_{k}^{+}$and the current estimation error covariance matrix $P_{k}^{+}$to the time of the next observation $t_{k+1}$.

4. Set $k=k+1$

5. Read in the observation data set $\boldsymbol{Z}_{k}$.

6. Set $\hat{\boldsymbol{X}}_{k_{0}}^{+}=\hat{\boldsymbol{X}}_{k}^{-}$

7. Calculate the $H_{k}\left(\hat{\boldsymbol{X}}_{k_{i}}^{+}\right)$matrix.

8. Compute the IEKF gain matrix $K_{k_{i}}$ and update the state estimate vector $\hat{\boldsymbol{X}}_{k}^{-}$.

9. Repeat steps 7 and 8 until the state estimate vector $\hat{\boldsymbol{X}}_{k_{i}}^{+}$no longer improves.

10. Update the estimation error covariance matrix $P_{k}^{-}$.

11. Repeat steps 3 - 10 until all of the observation data are processed.

The Gim-Alfriend system dynamics equations described in chapter 2 are now combined with the observation and the IEKF equations in this chapter to solve the relative orbit estimation problem for the satellites formation flights. 


\subsection{Numerical Evaluation}

In this numerical evaluation we demonstrate the performance of an iterated extended Kalman filter to determine the relative orbit states of the deputy satellites in close and far formation flights. We begin by synthetically generating a time history of radar observation data for different deputy satellites. To this end, we execute the following sequence of steps in our numerical simulation for each pair of the formation flying chief and deputy satellites.

1. We numerically integrate the equations of motion of both chief and deputy satellites in the Earth-centered-inertial (ECI) reference frame with $J_{2}$ to $J_{6}$ gravitational perturbations included.

2. Utilizing the direction cosine matrix $[\mathcal{O N}]$ defined by equation $(2.34 \mathrm{a})$, we transform the chief and deputy satellites position vectors from the ECI frame to the chief LVLH rectilinear frame at each simulation time step $t_{k}$.

3. In the chief LVLH rectilinear frame, we subtract the chief position vector from the deputy position vector to obtain the deputy satellite slant range vector $\boldsymbol{\rho}_{k}$.

4. We employ equation (3.3) to obtain the radar true measurements data and then add zero-mean white Gaussian noise to generate the observation data $\boldsymbol{Z}_{k}$.

After artificially creating the noisy measurement data, we carry out the next three steps to assess the usefulness of an IEKF in reliably estimating the relative orbit states of the deputy satellites.

4. Utilizing the chief and deputy satellites position and velocity vectors in the ECI frame, we compute directly the deputy satellite actual relative position and velocity vectors in the chief LVLH curvilinear frame.

5. We employ an IEKF to estimate the relative motion of the deputy satellites in the chief LVLH curvilinear frame. 
6. We compare the actual relative position and velocity vectors from step 4 with the estimated relative position and velocity vectors from step 5 .

\subsubsection{Simulation Test Plans}

We utilize the same three different simulation test cases devised in chapter 2 to investigate the performance of our proposed relative orbit determination method. Recall that in each test case the relative motions of two deputy satellites with respect to a chief satellite are examined. In test cases 1 and 2 , the chief and two deputy satellites fly in close formation and in test case 3, a far formation flight of satellites is inspected. The numerical integrations of both chief and deputy satellites equations of motions in the ECI reference frame is again performed in 0.5 seconds time steps. For consistancy, the application of an IEKF to estimate the relative orbit state of the deputy satellites in the chief LVLH curvilinear frame is also performed in 0.5 seconds time steps. We assume the chief satellite in every test case is equipped with an active phased array radar (APAR) with a maximum range of $400 \mathrm{~km}$ and it can track simultaneously at least 2 deputy satellites. An APAR measurement errors are often described statistically by a three - dimensional zero-mean Gaussian probability density function. For a typical APAR, the standard deviation of the range measurement error is $\sigma_{\rho}=25$ meters, and a standard deviation of the angle measurements are $\sigma_{a z}=\sigma_{e l}=0.2$ degrees. Thus we set the measurement noise covariance matrix $R$ to

$$
R=\left[\begin{array}{ccc}
(25)^{2} & 0 & 0 \\
0 & \left(0.2 \times \frac{\pi}{180}\right)^{2} & 0 \\
0 & 0 & \left(0.2 \times \frac{\pi}{180}\right)^{2}
\end{array}\right]
$$


Figure 3.2 shows an example of synthetically generated Gaussian noise that are added to the chief APAR observations of a deputy satellite. In Figure 3.2a, the real values of a Gaussian noise and its representative probability density function for a typical APAR range measurement errors is displayed. We note that as a Gaussian noise, $68.3 \%$ of the probability weight is contained within the standard deviation $\sigma_{\rho}=25$ meters band to each side of the mean. Figures $3.2 \mathrm{~b}$ and $3.2 \mathrm{c}$ similarly show the azimuth and elevation measurement errors real values in degrees along with their representative probability density functions for a typical APAR.
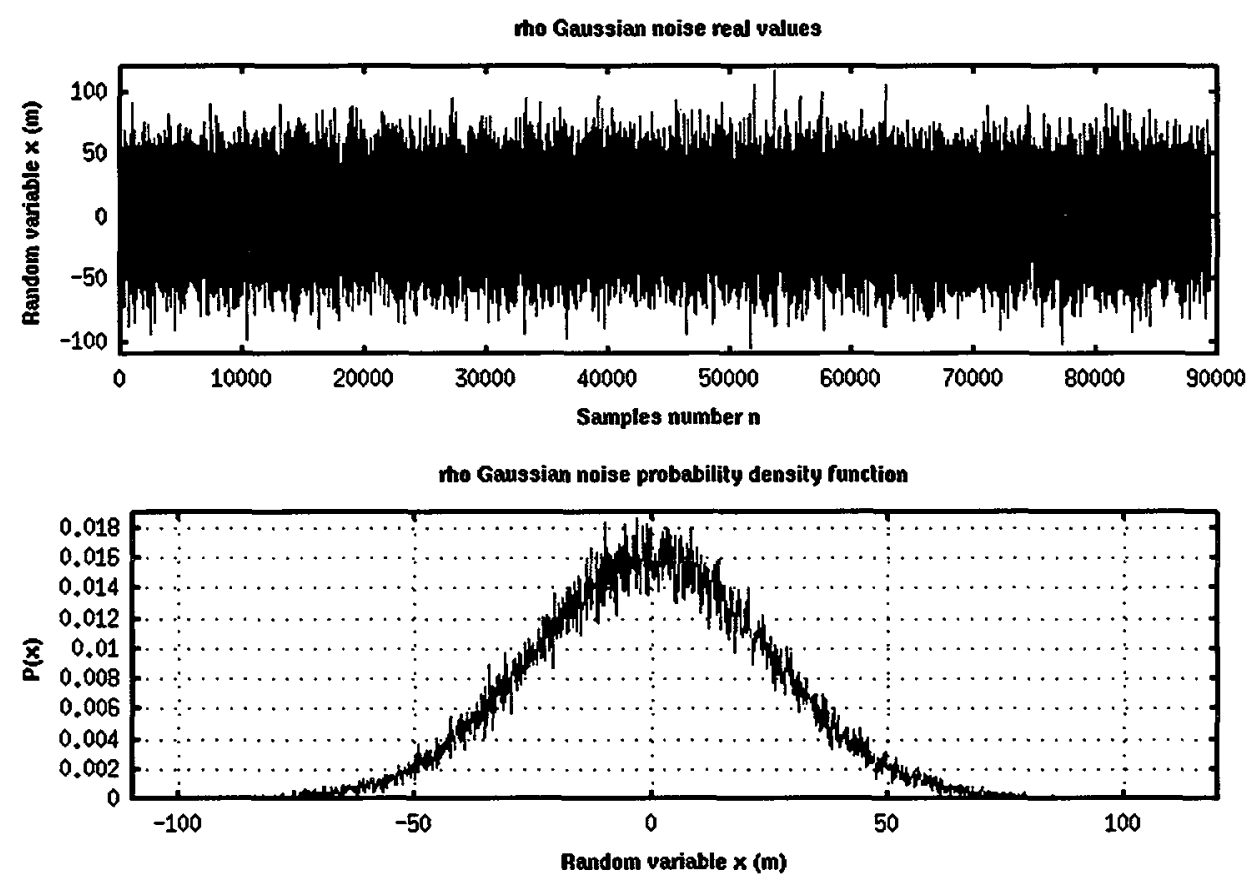

(a) $\rho$ Gaussian noise real values and probability density function 

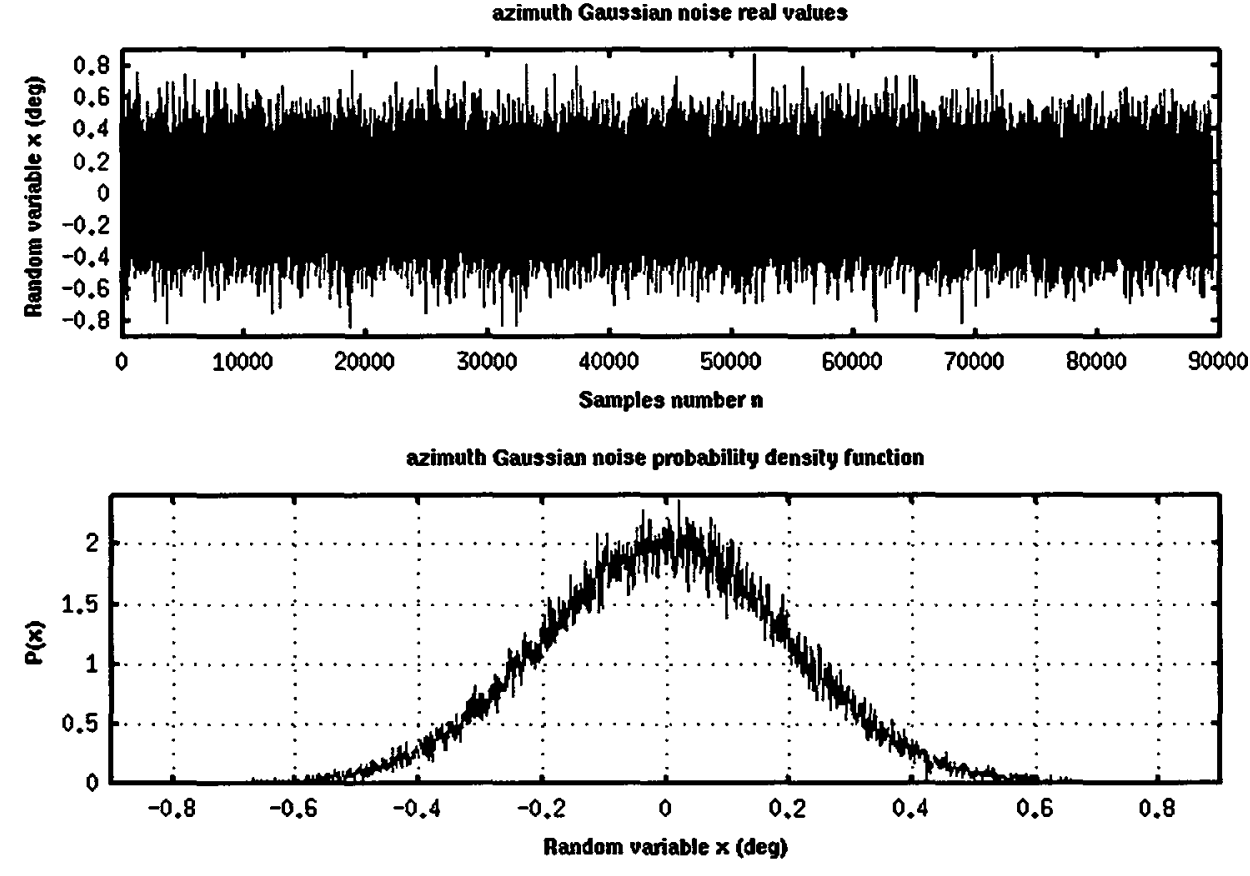

(b) $a z$ Gaussian noise real values and probability density function
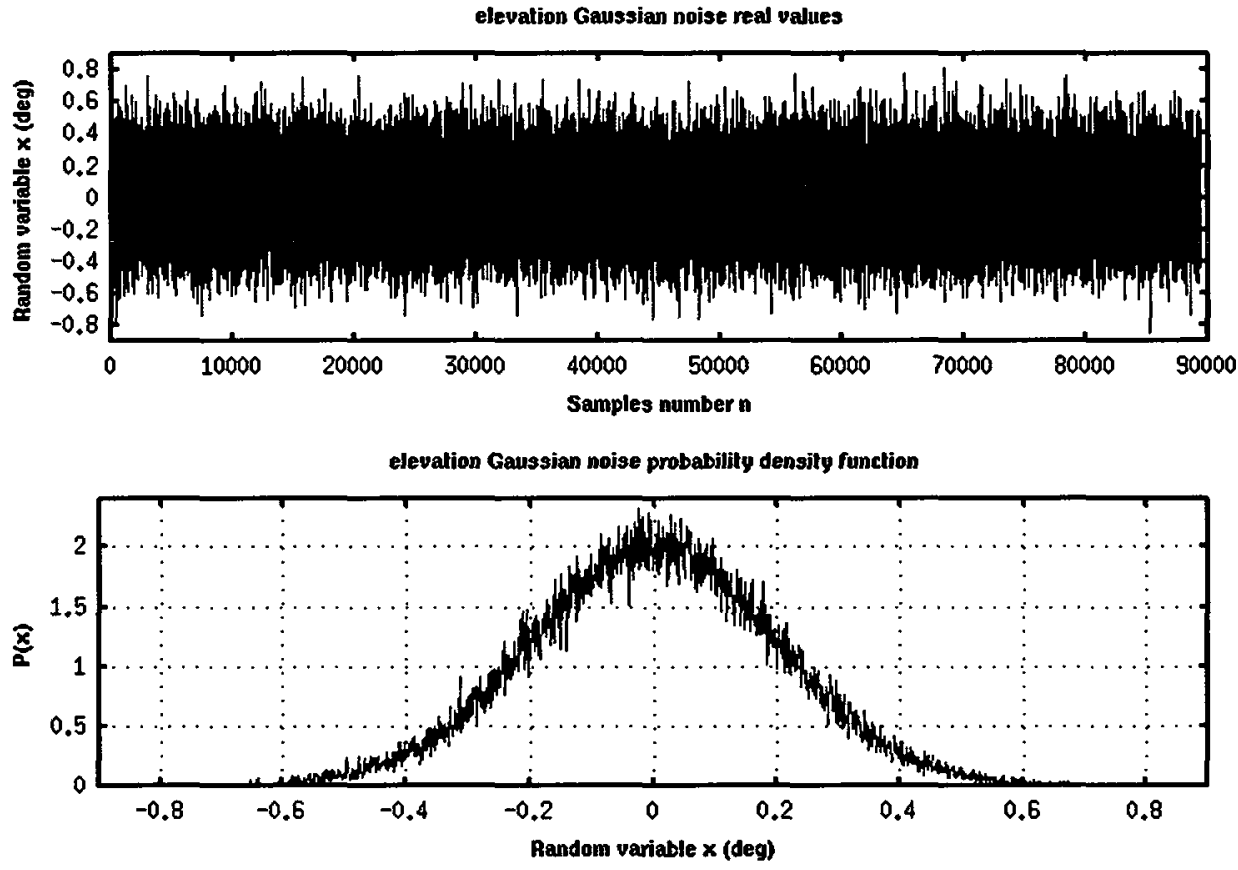

(c) $e l$ Gaussian noise real values and probability density function

Figure 3.2: Illustration of an example of Gaussian noise added to slant range vector observations 
We set the initial estimation error covariance matrix $P_{0}^{+}$to the following diagonal matrix for all the simulation test cases.

$$
P_{0}^{+}=\operatorname{diag}\left[(1 \mathrm{e}-3)^{2},(1 \mathrm{e}-6)^{2},(1 \mathrm{e}-3)^{2},(1 \mathrm{e}-6)^{2},(1 \mathrm{e}-3)^{2},(1 \mathrm{e}-6)^{2}\right]
$$

The system noise covariance matrix $Q$ is also set to the following diagonal matrix for all the simulation test cases. Note that the $Q$ matrix diagonal elements were chosen by trial and error.

$$
Q=\operatorname{diag}[1 \mathrm{e}-7,1 \mathrm{e}-14,1 \mathrm{e}-7,1 \mathrm{e}-14,1 \mathrm{e}-7,1 \mathrm{e}-14]
$$

Since the proposed relative orbit determination method in this research relies on a radar observations $\boldsymbol{Z}$ in the chief satellite LVLH rectilinear frame, we must make sure the approximation (3.4) always remains valid during the complete simulated flight times. Hence in every test case we compute and display the difference between the deputy satellite slant range vector components $\left(\rho_{x}, \rho_{y}, \rho_{z}\right)^{T}$ and the corresponding relative orbit position vector components $(x, y, z)^{T}$.

We now present the simulation results for the three test cases one at the time. 


\subsubsection{Deputy Satellites Relative Orbit Determinations for Test Case 1}

The initial conditions for the chief and two deputy satellites in this test case were given in tables 2.1 and 2.2. The test case 1 simulation is run for a duration of 15 chief/deputy orbital periods which is equal to 89310 seconds or almost 24.81 hours. Figures 3.3 and 3.4 display the difference between the rectilinear and the curvilinear relative position vector components of the deputy satellites 1 and 2 respectively. It is clear from Figure 3.3 that the maximum absolute value of error belongs to the radial component of the relative position vector of deputy satellite 1 and it is almost less than 2.1 centimeters. Similarly in Figure 3.4 for the deputy satellite 2, the maximum absolute value of error belongs to the radial component of the relative position vector and still is on the order of a few centimeters. It is less than 4 centimeters after 24 hours which is very insignificant for most applications. Therefore approximation (3.4) is valid during test case 1 entire simulated flight times. 


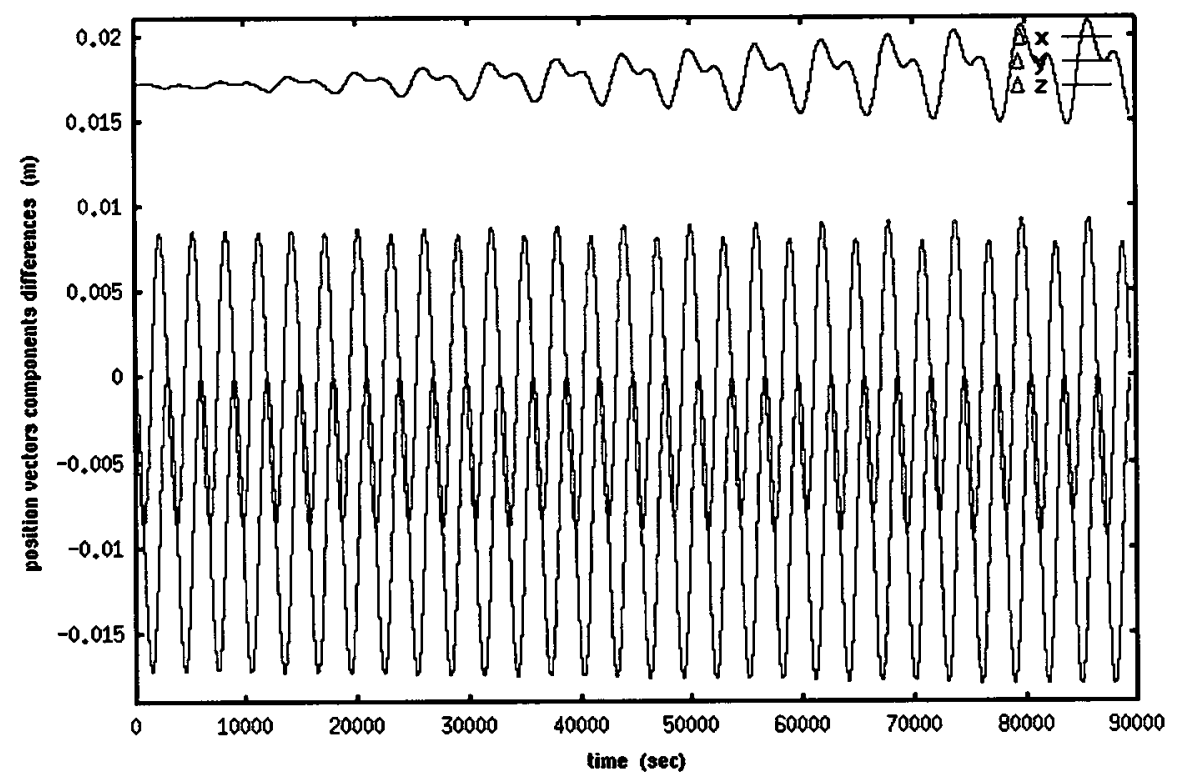

Figure 3.3: Illustration of the deputy satellite 1 rectilinear versus curvilinear relative position vector differences in the test case 1

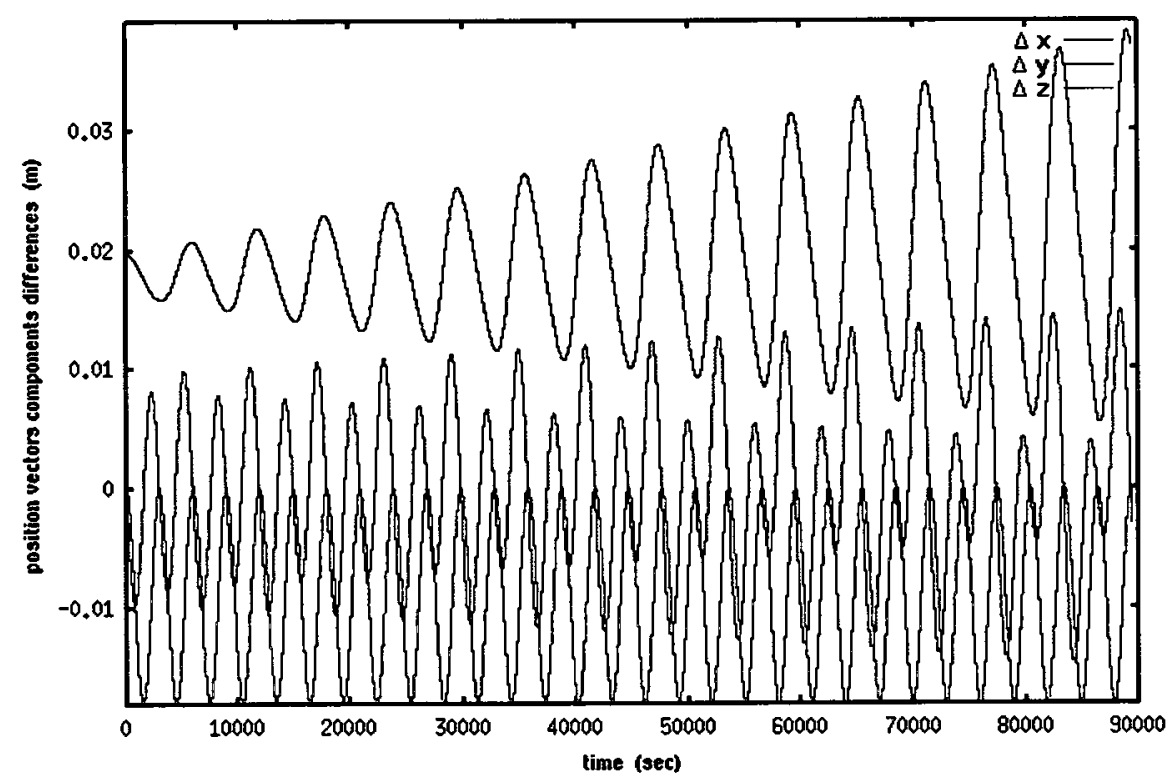

Figure 3.4: Illustration of the deputy satellite 2 rectilinear versus curvilinear relative position vector differences in the test case 1 


\section{Simulation results for the deputy satellite 1}

Figure 3.5 shows the time history of the deputy satellite 1 noisy observations collected by the chief satellite radar. Figure 3.5a displays the slant range vector $\rho$ distance and Figure 3.5b exhibits the azimuth $a z$ and elevation $e l$ angles. The slant range vector $\rho$ time history clearly shows a secular growth in the distance between the chief satellite and the deputy satellite 1. Figure 3.6 displays the deputy satellite 1 actual relative position and velocity vector components in the chief LVLH curvilinear frame and compare it against the estimated relative position and velocity vectors components that are computed by our iterated extended Kalman filter. Figure 3.7 demonstrates the performance of our proposed IEKF in accomplishing the relative orbit determination task for the deputy satellite 1. Figure 3.7a shows that the maximum absolute value of the IEKF estimation errors for the deputy satellite 1 relative position vector components is less than 1.5 meters for the entire simulated flight times. We also notice that there is no indication of secular growth in the estimation errors for the radial, in-track, and cross-track relative position vector components. If we compare Figure 3.7a on page 111 with Figure 2.8a on page 66, we clearly see that the progressively increasing in-track errors do no exist and specially after the first orbit, all relative position vector components errors are confined within a band of -1.1 to 0.7 meters. Turning our attention to Figure $3.7 \mathrm{~b}$, we note that the maximum absolute value of the IEKF estimation errors for the deputy satellite 1 relative velocity vector components is less 2.5 millimeters per second and similarly there is no sign of secular growth in the estimation errors during 24.81 hours of simulated flight times. Comparing Figure $3.7 \mathrm{~b}$ on page 111 with Figure $2.8 \mathrm{~b}$ on page 66 easily displays that after the first orbit, the proposed IEKF has limited the relative velocity vector components errors to \pm 0.7 millimeter per second.

We infer from these test case 1 simulation results that the proposed IEKF performs very good in estimating the deputy satellite 1 relative position and velocity vectors. 


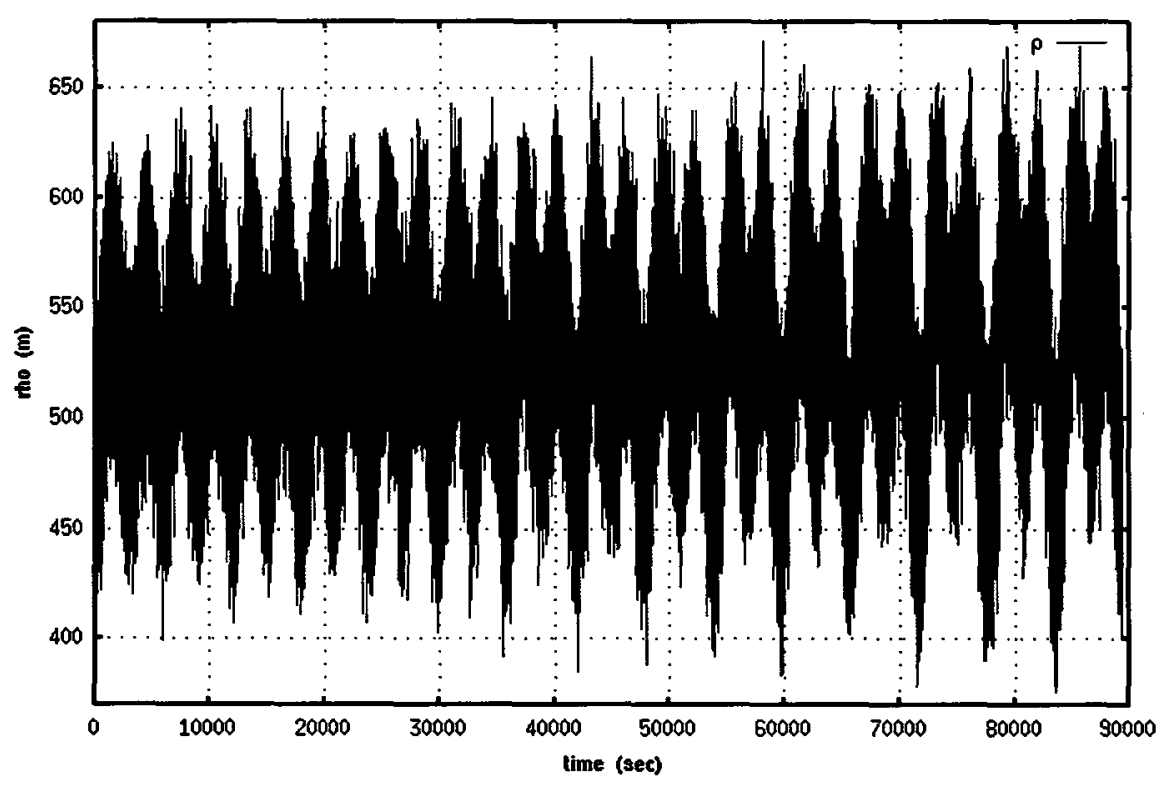

(a) Slant range vector $\rho$ observation of the deputy satellite 1

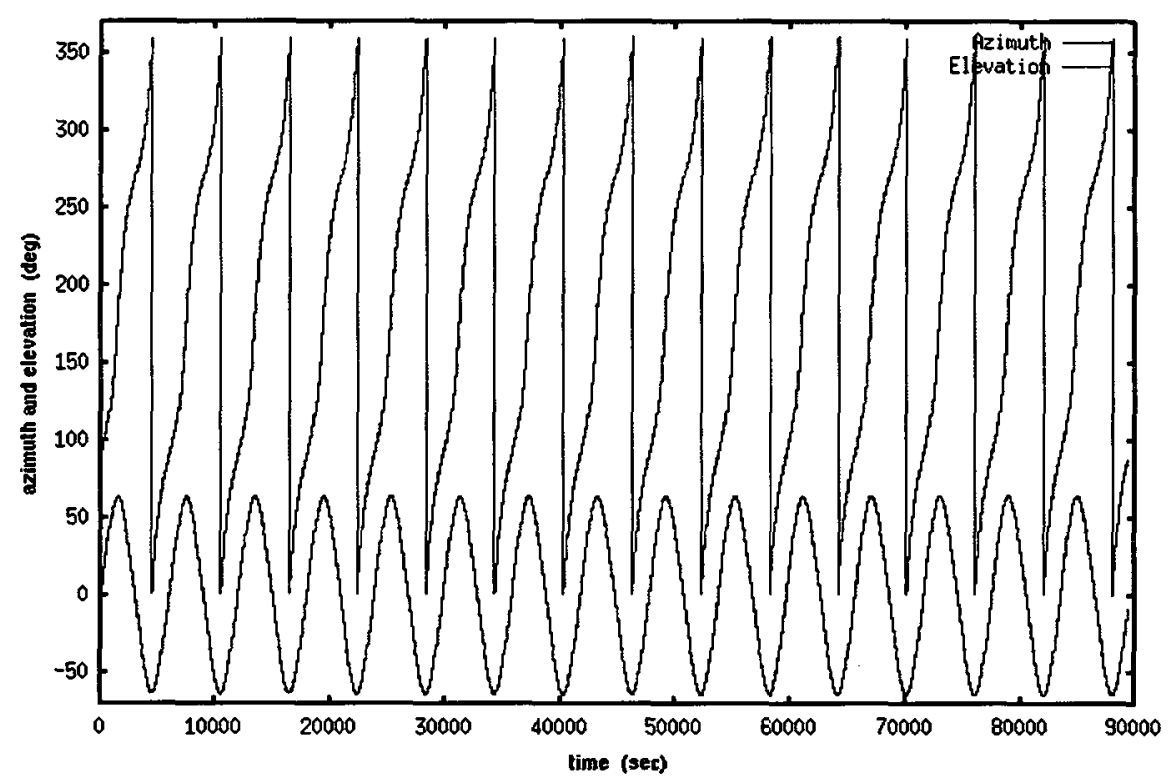

(b) Slant range vector $a z$ and $e l$ observations of the deputy satellite 1

Figure 3.5: Illustration of the deputy satellite 1 slant range vector observations data in the test case 1 

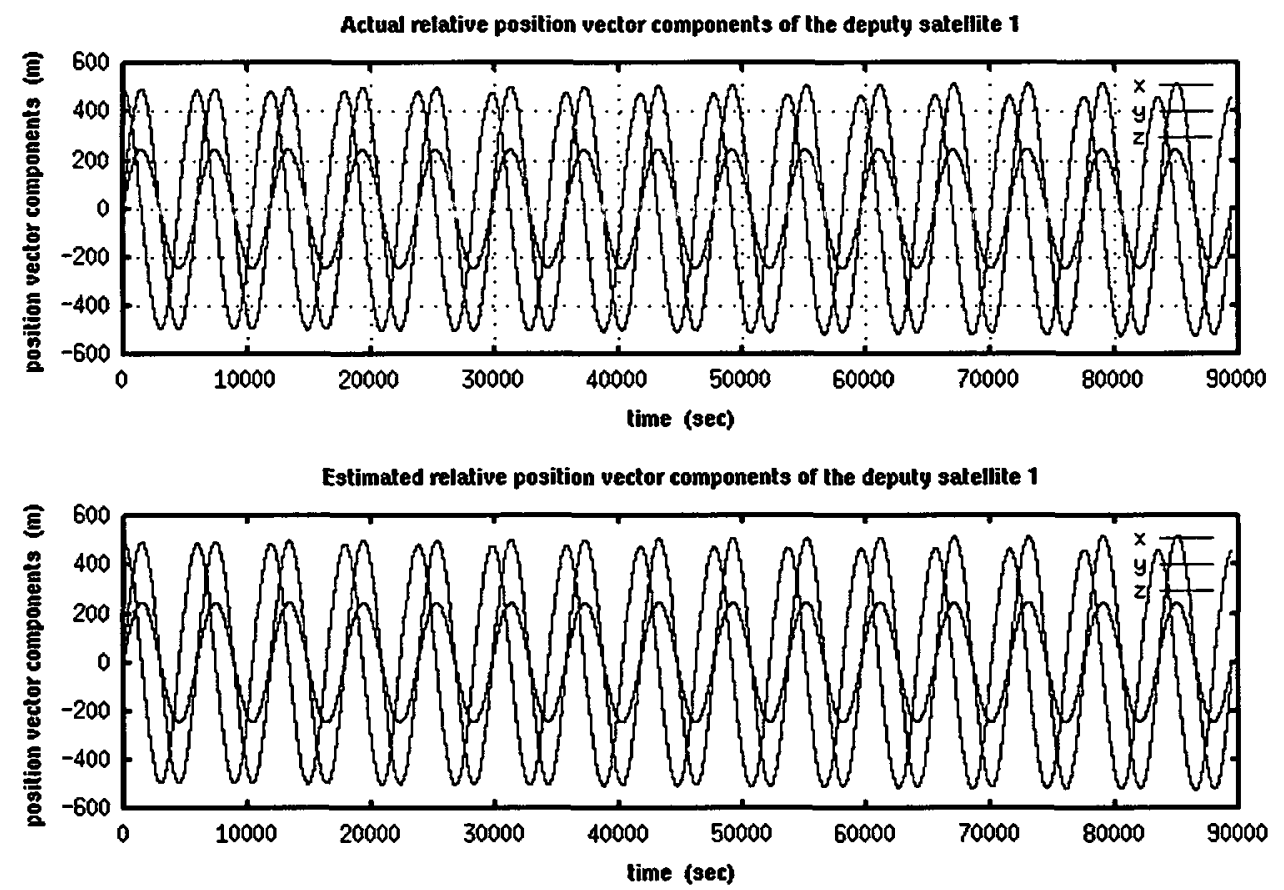

(a) Actual and estimated relative position vectors components of the deputy satellite 1
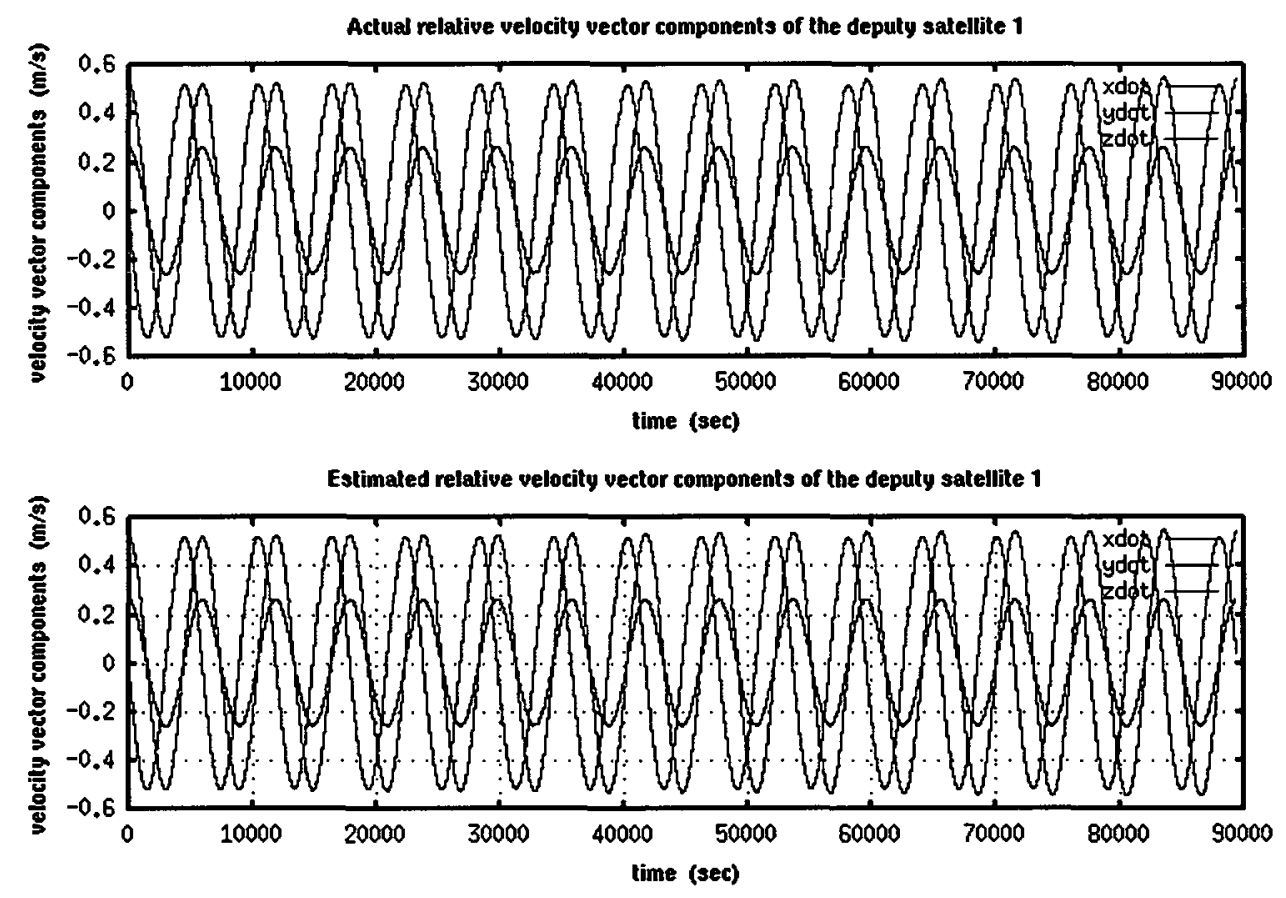

(b) Actual and estimated relative velocity vectors components of the deputy satellite 1

Figure 3.6: Illustration of the deputy satellite 1 actual/estimated relative position and velocity vectors components in the test case 1 


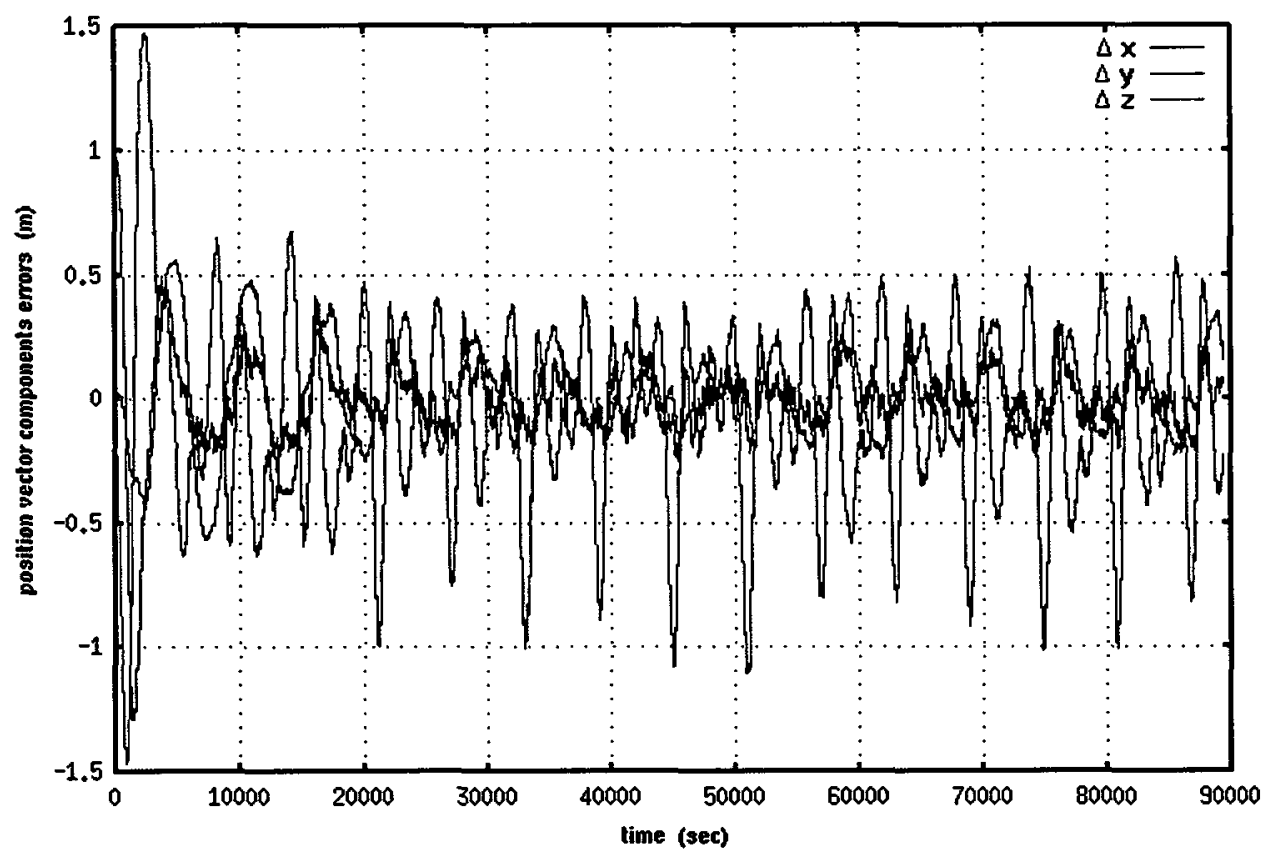

(a) Relative position vector components estimation errors of the deputy satellite 1

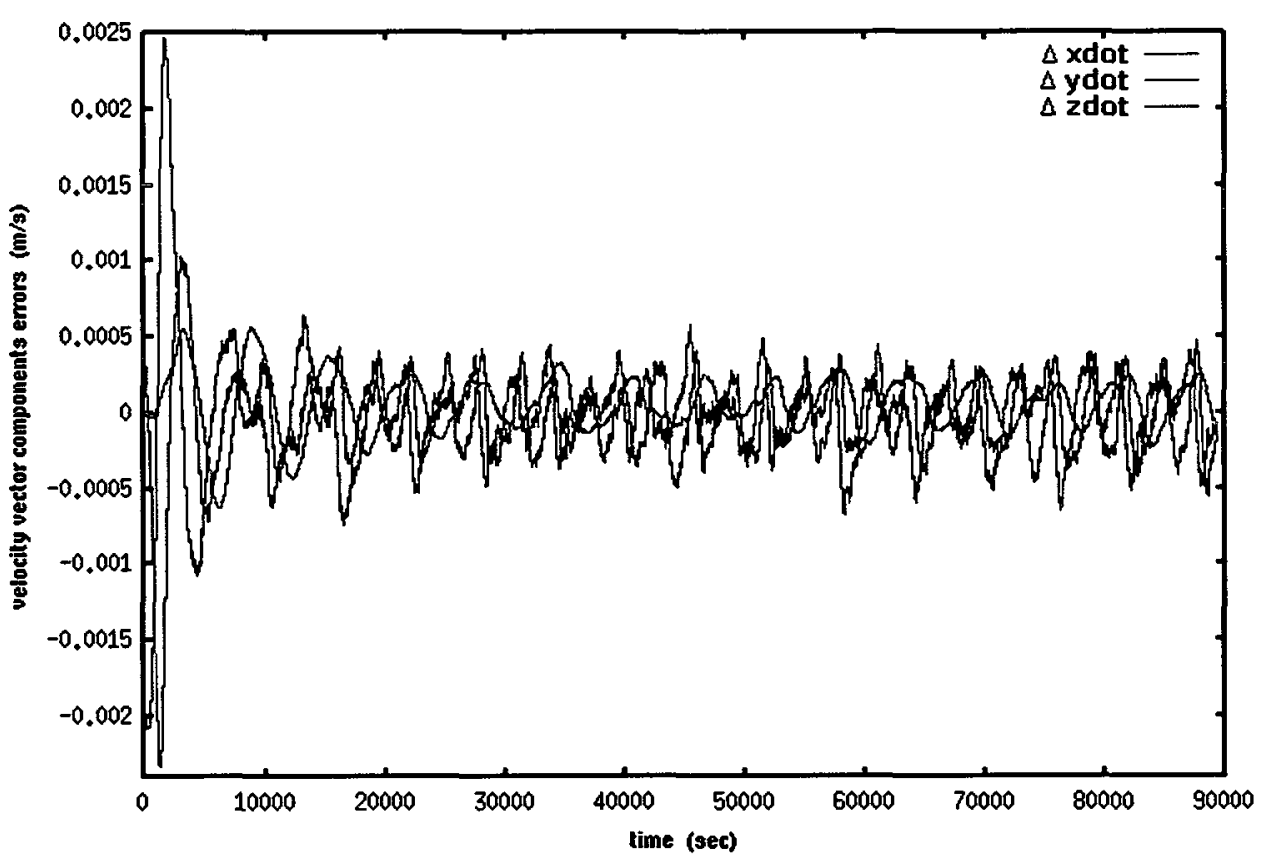

(b) Relative velocity vector components estimation errors of the deputy satellite 1

Figure 3.7: Illustration of the deputy satellite 1 relative position and velocity estimation errors in the test case 1 


\section{Simulation results for the deputy satellite 2}

Figure 3.8 shows the time history of the deputy satellite 2 noisy observations collected by the chief satellite radar. Figure 3.8a displays the slant range vector $\rho$ distance and Figure $3.8 \mathrm{~b}$ exhibits the azimuth $a z$ and elevation $e l$ angles. The slant range vector $\rho$ time history clearly shows a secular growth in the distance between the chief satellite and the deputy satellite 2. Figure 3.9 displays the deputy satellite 2 actual relative position and velocity vector components in the chief LVLH curvilinear frame and compare it against the estimated relative position and velocity vectors components that are computed by our iterated extended Kalman filter. Figure 3.10 demonstrates the performance of our proposed IEKF in accomplishing the relative orbit determination task for the deputy satellite 2 . Figure 3.10 a shows that the maximum absolute value of the IEKF estimation errors for the deputy satellite 2 relative position vector components is less than 1.6 meters for the entire simulated flight times. We also notice that there is no indication of secular growth in the estimation errors for the radial, in-track, and cross-track relative position vector components. If we compare Figure 3.10 a on page 115 with Figure 2.10 a on page 69 , we clearly see that the progressively increasing in-track errors do no exist and specially after the first orbit, all relative position vector components are confined within a band of \pm 0.75 meters. Turning our attention to Figure $3.10 \mathrm{~b}$, we note that the maximum absolute value of the IEKF estimation errors for the deputy satellite 2 relative velocity vector components is less 2.3 millimeters per second and similarly there is no sign of secular growth in the estimation errors during 24.81 hours of simulated flight times. Comparing Figure $3.10 \mathrm{~b}$ on page 115 with Figure $2.10 \mathrm{~b}$ on page 69 easily displays that after the first orbit, the proposed IEKF has limited the relative velocity vector components errors to \pm 0.75 millimeters per second.

We infer from these test case 1 simulation results that the proposed IEKF performs very good in estimating the deputy satellite 2 relative position and velocity vectors. 


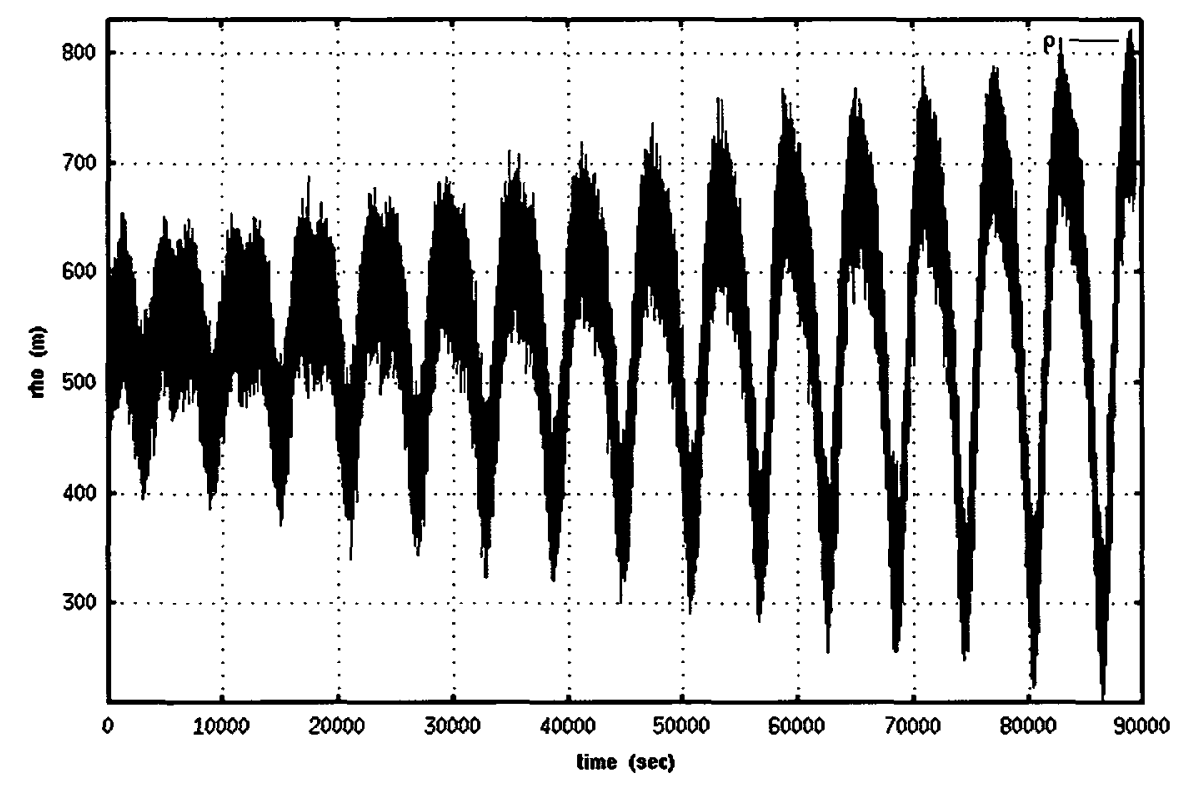

(a) Slant range vector $\rho$ observation of the deputy satellite 2

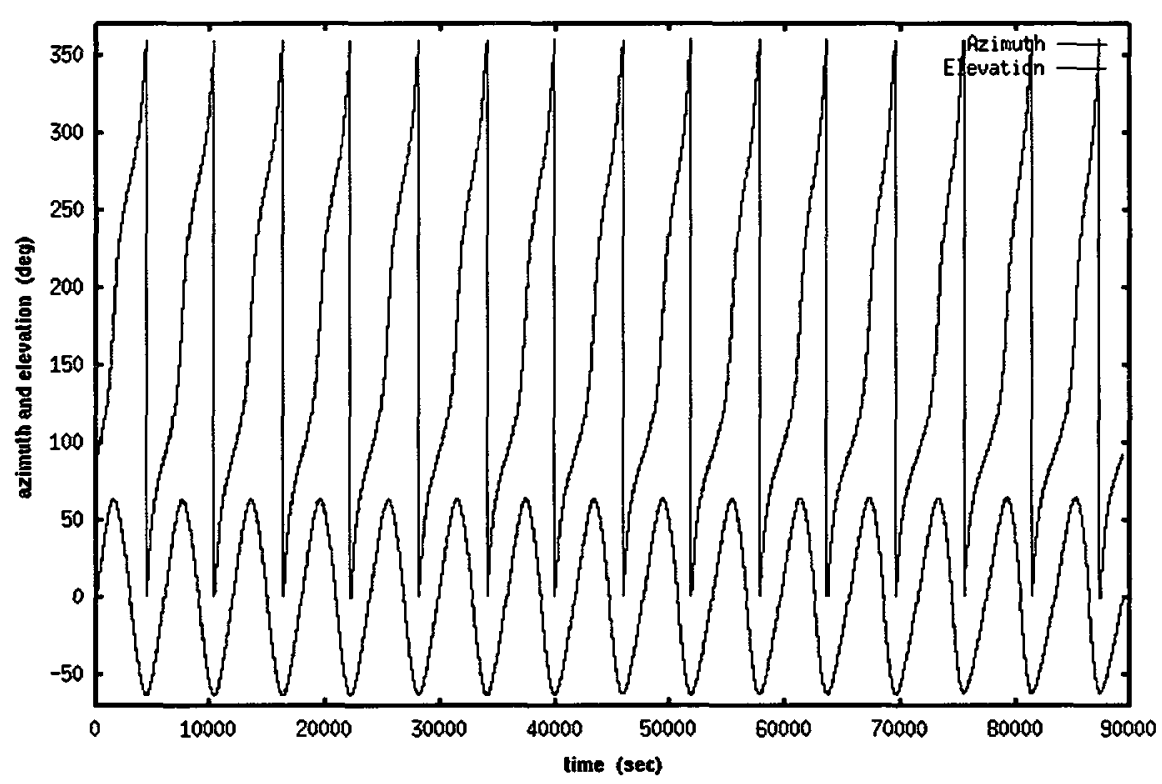

(b) Slant range vector $a z$ and $e l$ observations of the deputy satellite 2

Figure 3.8: Illustration of the deputy satellite 2 slant range vector observations data in the test case 1 

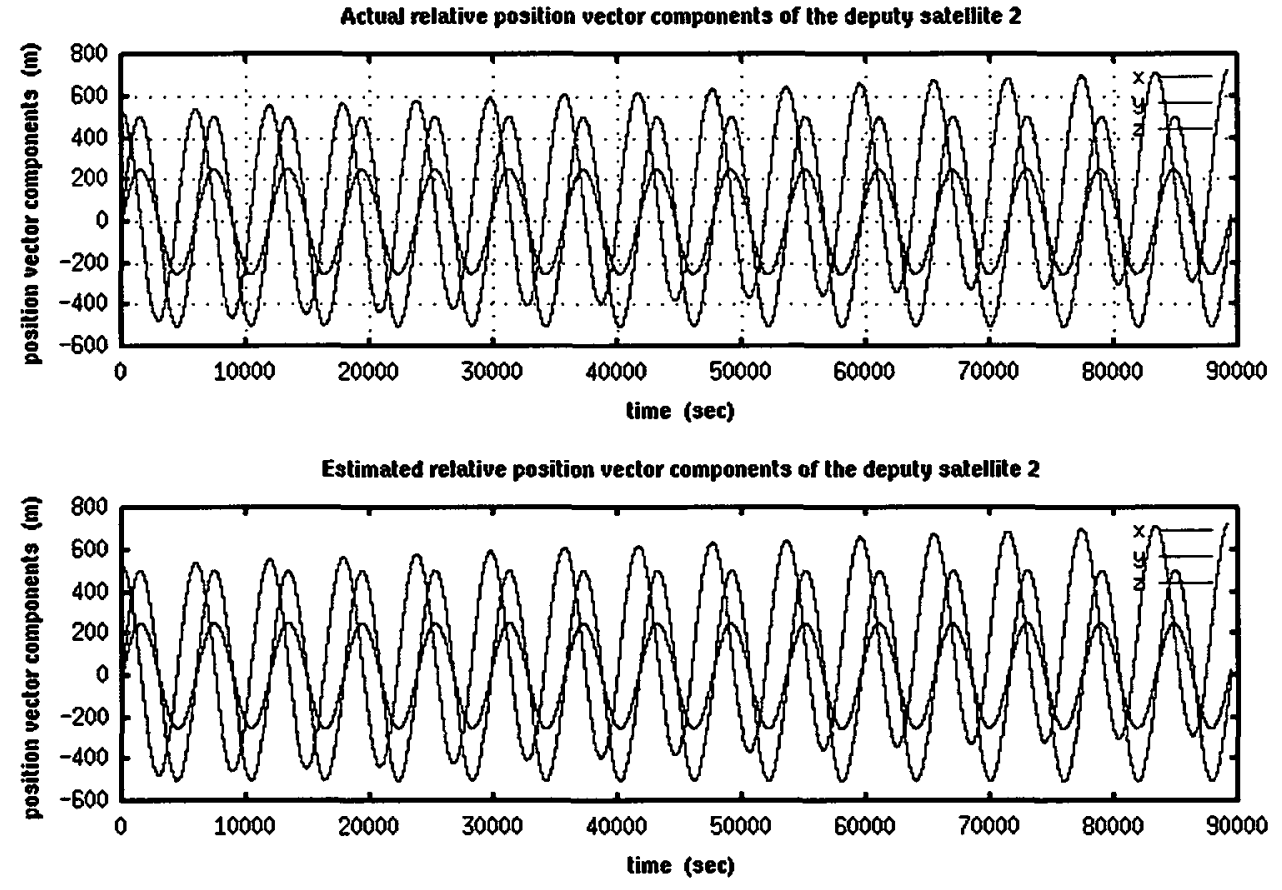

(a) Actual and estimated relative position vectors components of the deputy satellite 2
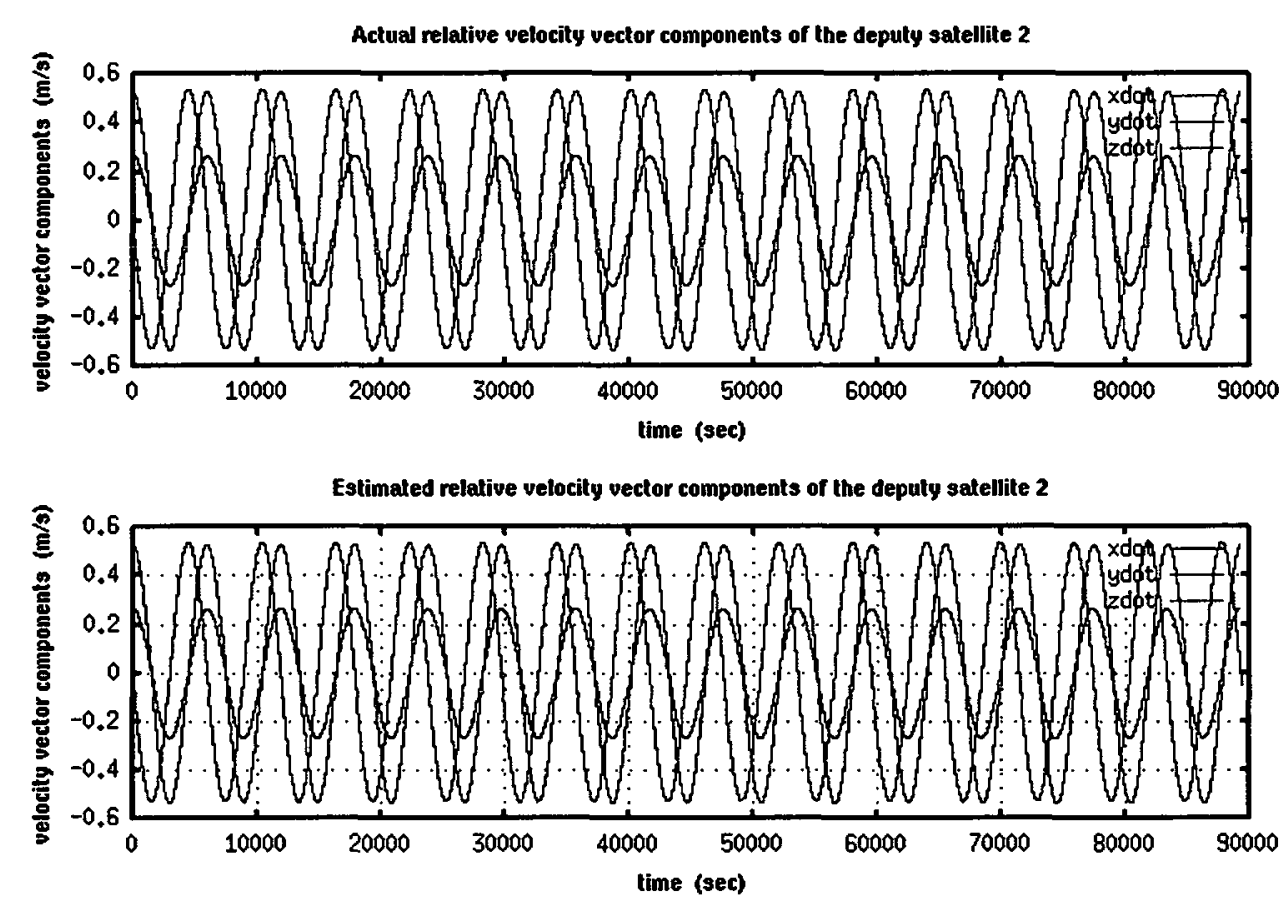

(b) Actual and estimated relative velocity vectors components of the deputy satellite 2

Figure 3.9: Illustration of the deputy satellite 2 actual/estimated relative position and velocity vectors components in the test case 1 


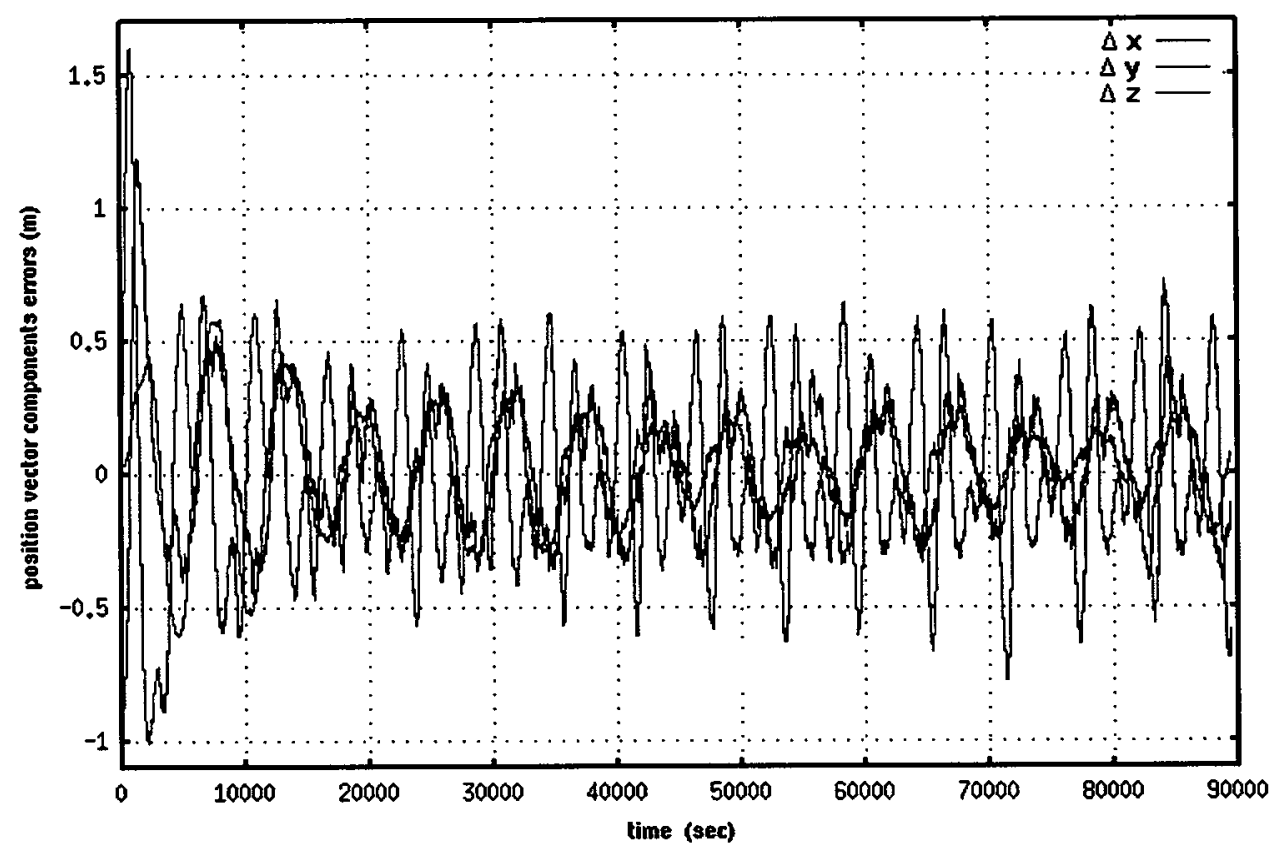

(a) Relative position vector components estimation errors of the deputy satellite 2

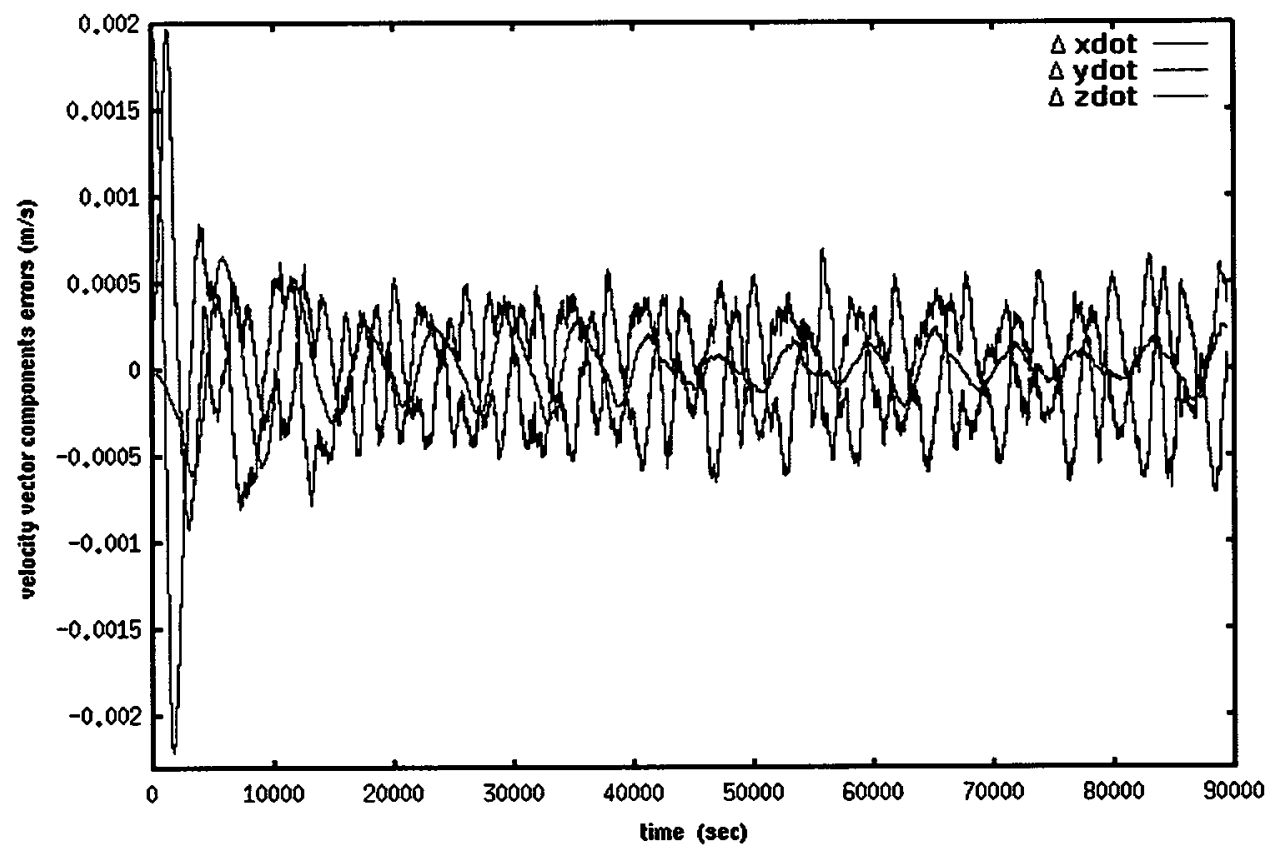

(b) Relative velocity vector components estimation errors of the deputy satellite 2

Figure 3.10: Illustration of the deputy satellite 2 relative position and velocity estimation errors in the test case 1 


\subsubsection{Deputy Satellites Relative Orbit Determinations for Test Case 2}

The initial conditions for the chief and two deputy satellites in this test case were given in tables 2.3 and 2.4. The test case 2 simulation is run for a duration of 15 chief/deputy orbital periods which is equal to 83715 seconds or almost 23.25 hours. Figures 3.11 and 3.12 display the difference between the rectilinear and the curvilinear relative position vector components of the deputy satellites 1 and 2 respectively. It is clear from Figure 3.11 that the maximum absolute value of difference belongs to the radial component of the relative position vector of deputy satellite 1 and it is almost 4.6 centimeters. Similary in Figure 3.12 for the deputy satellite 2, the maximum absolute value of difference belongs to the radial component of the relative position vector and still is on the order of a few centimeters. It is less than 4 centimeters after 24 hours which is very insignificant for most applications. Therefore approximation (3.4) is valid during test case 2 entire simulated flight times. 


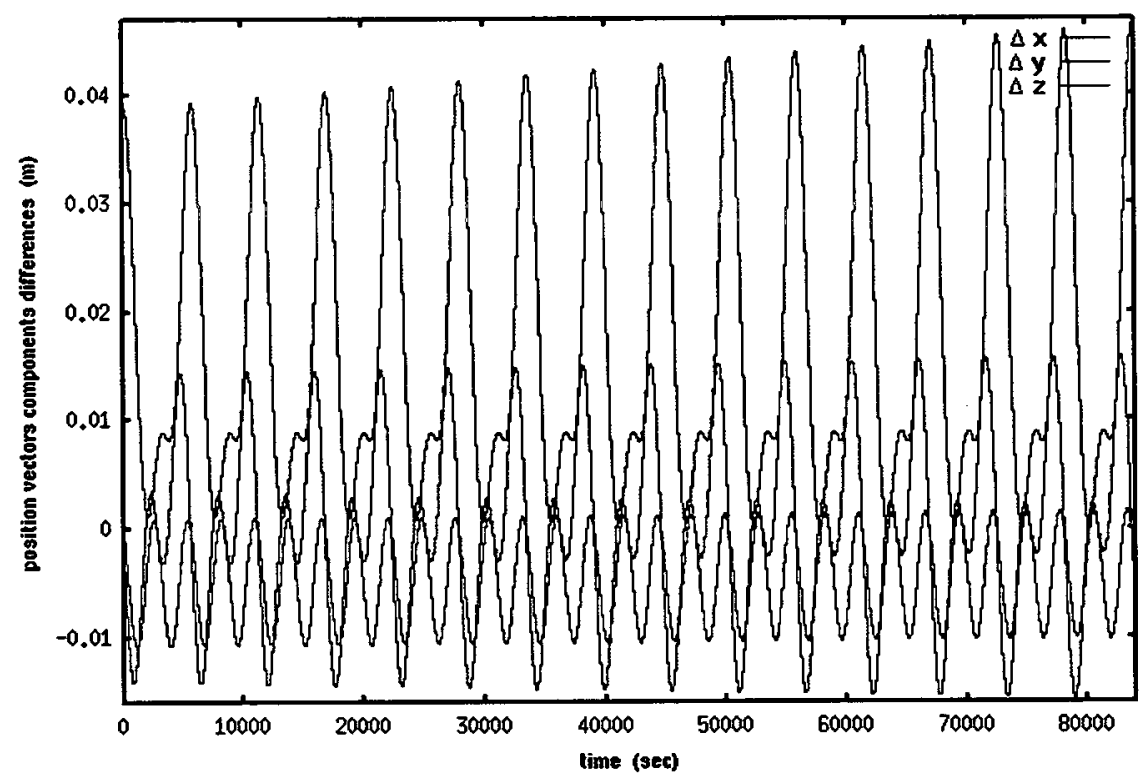

Figure 3.11: Illustration of the deputy satellite 1 rectilinear versus curvilinear relative position vector differences in the test case 2

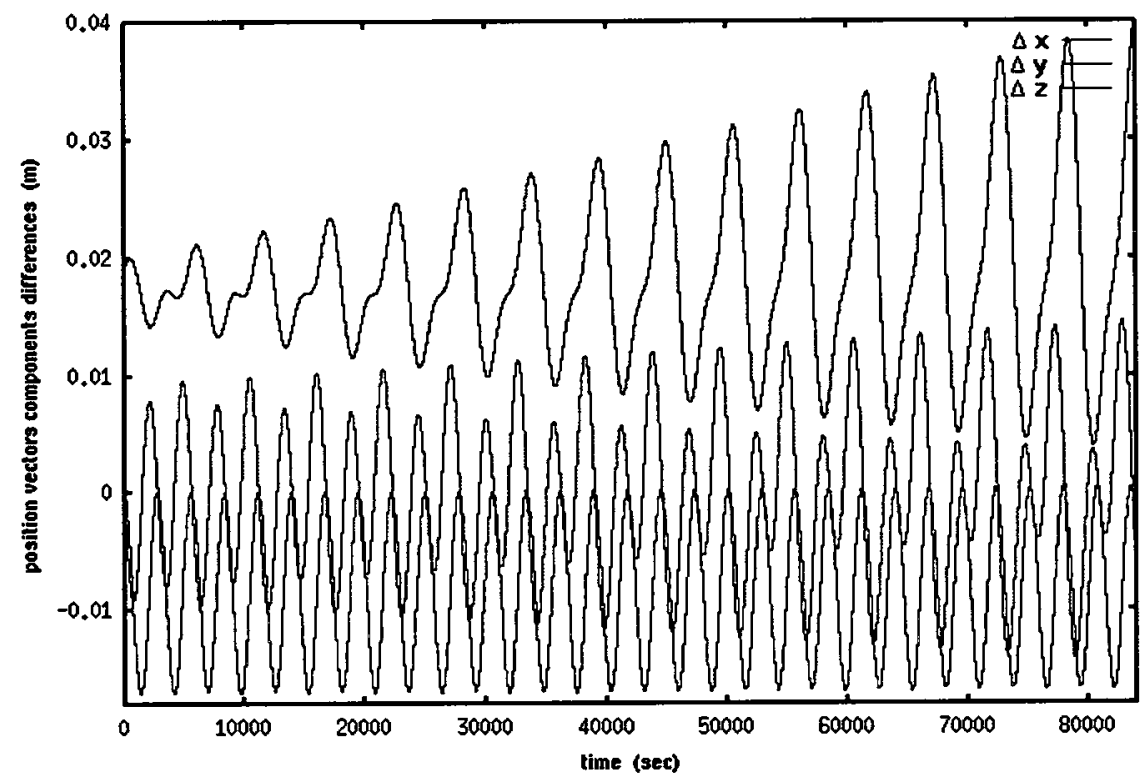

Figure 3.12: Illustration of the deputy satellite 2 rectilinear versus curvilinear relative position vector differences in the test case 2 


\section{Simulation results for the deputy satellite 1}

Figure 3.13 shows the time history of the deputy satellite 1 noisy observations collected by the chief satellite radar. Figure 3.13a displays the slant range vector $\rho$ distance and Figure 3.13b exhibits the azimuth $a z$ and elevation $e l$ angles. The slant range vector $\rho$ time history clearly shows a secular growth in the distance between the chief satellite and the deputy satellite 1. Figure 3.14 displays the deputy satellite 1 actual relative position and velocity vector components in the chief LVLH curvilinear frame and compare it against the estimated relative position and velocity vectors components that are computed by our iterated extended Kalman filter. Figure 3.15 demonstrates the performance of our proposed IEKF in accomplishing the relative orbit determination task for the deputy satellite 1. Figure $3.15 \mathrm{a}$ shows that the maximum absolute value of the IEKF estimation errors for the deputy satellite 1 relative position vector components is less than 2.7 meters for the entire simulated flight times. We also notice that there is no indication of secular growth in the estimation errors for the radial, in-track, and cross-track relative position vector components. If we compare Figure $3.15 \mathrm{a}$ on page 121 with Figure $2.13 \mathrm{a}$ on page 75 , we clearly see that the progressively increasing in-track errors do no exist and specially after the first two orbits, all relative position vector components errors are confined within a band of -1.5 to 0.5 meters. Turning our attention to Figure $3.15 \mathrm{~b}$, we note that the maximum absolute value of the IEKF estimation errors for the deputy satellite 1 relative velocity vector components is less 2.7 millimeters per second during 23.25 hours of simulated flight times. Comparing Figure 3.15b on page 121 with Figure $2.13 \mathrm{~b}$ on page 75 easily displays that after the first two orbits, the proposed IEKF has limited the relative velocity vector components errors to a band of -0.8 to 0.5 millimeters per second.

We infer from these test case 2 simulation results that the proposed IEKF performs very good in estimating the deputy satellite 1 relative position and velocity vectors. 


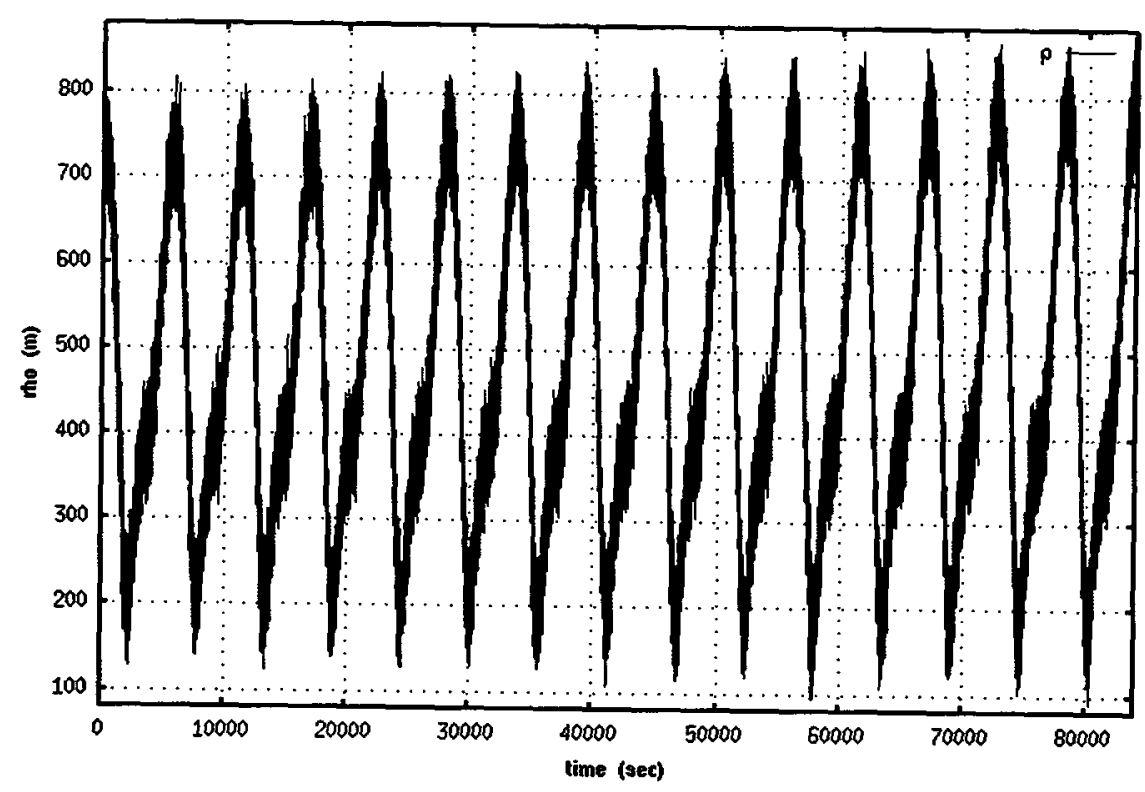

(a) Slant range vector $\rho$ observation of the deputy satellite 1

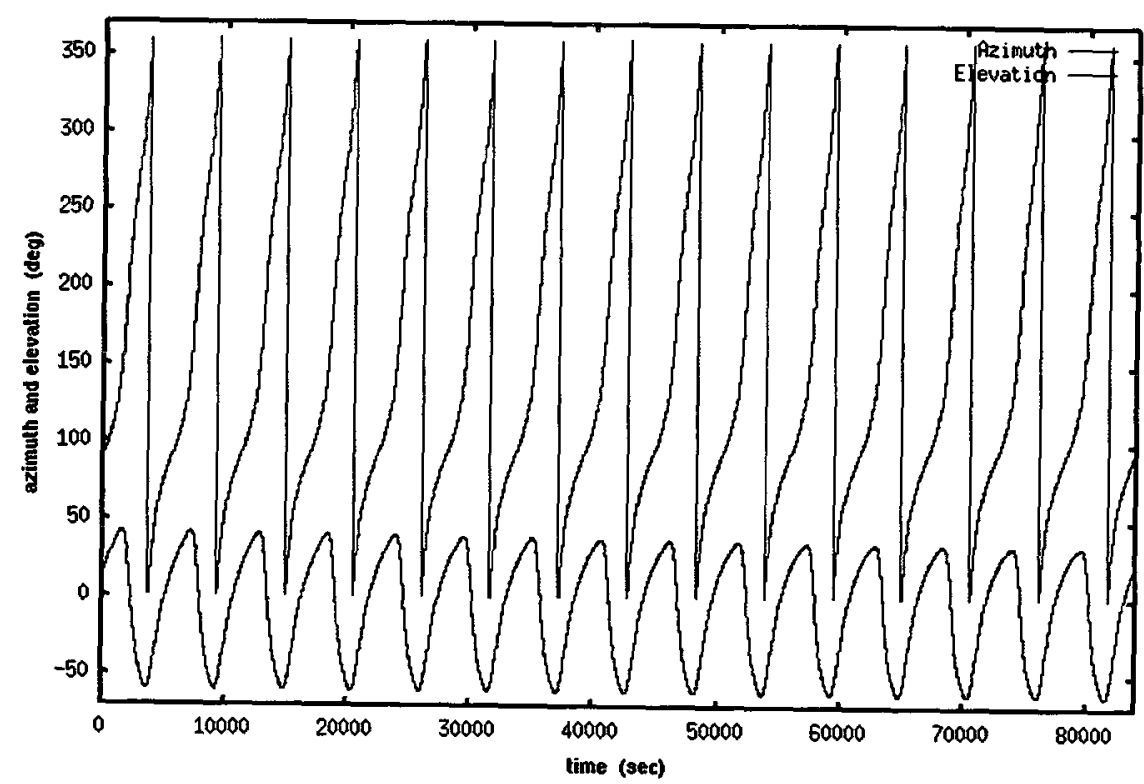

(b) Slant range vector $a z$ and $e l$ observations of the deputy satellite 1

Figure 3.13: Illustration of the deputy satellite 1 slant range vector observations data in the test case 2 

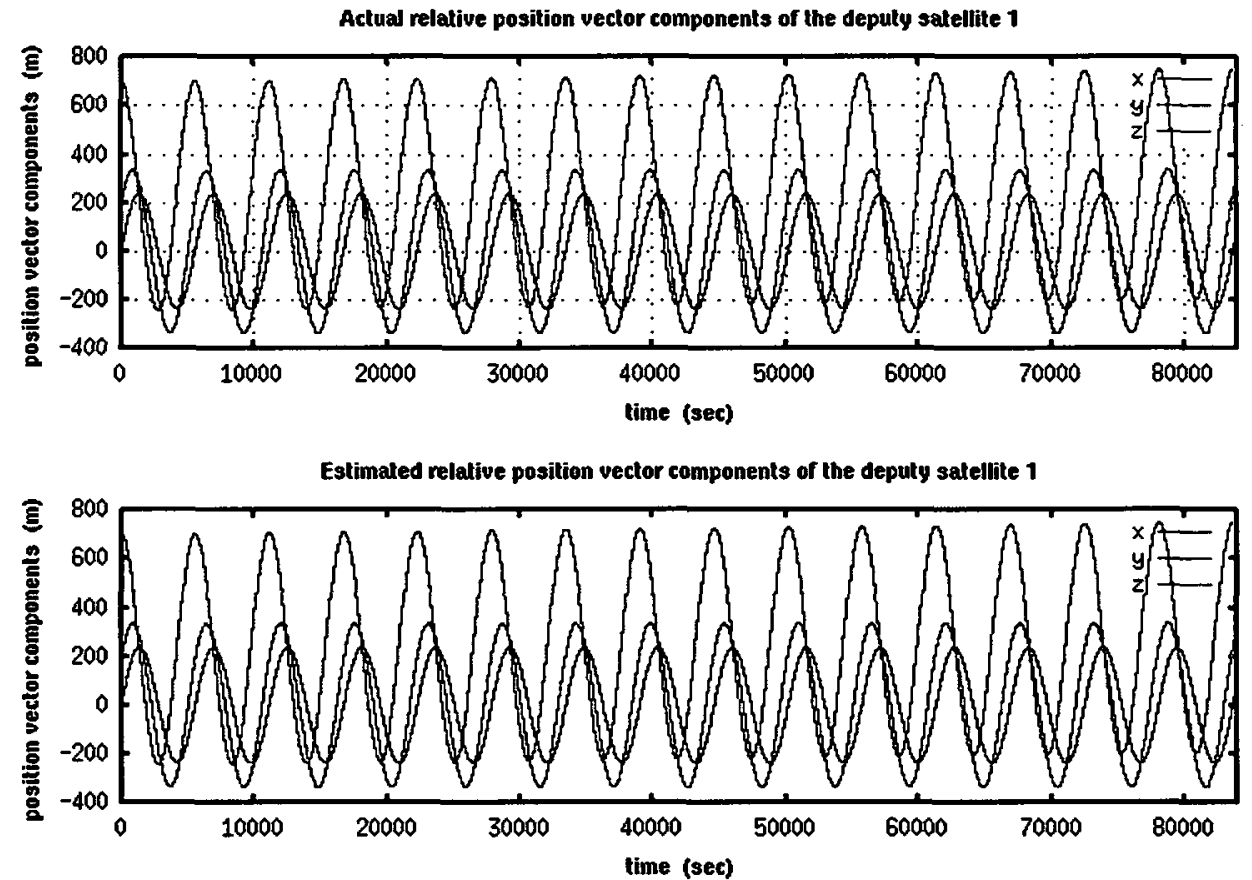

(a) Actual and estimated relative position vectors components of the deputy satellite 1
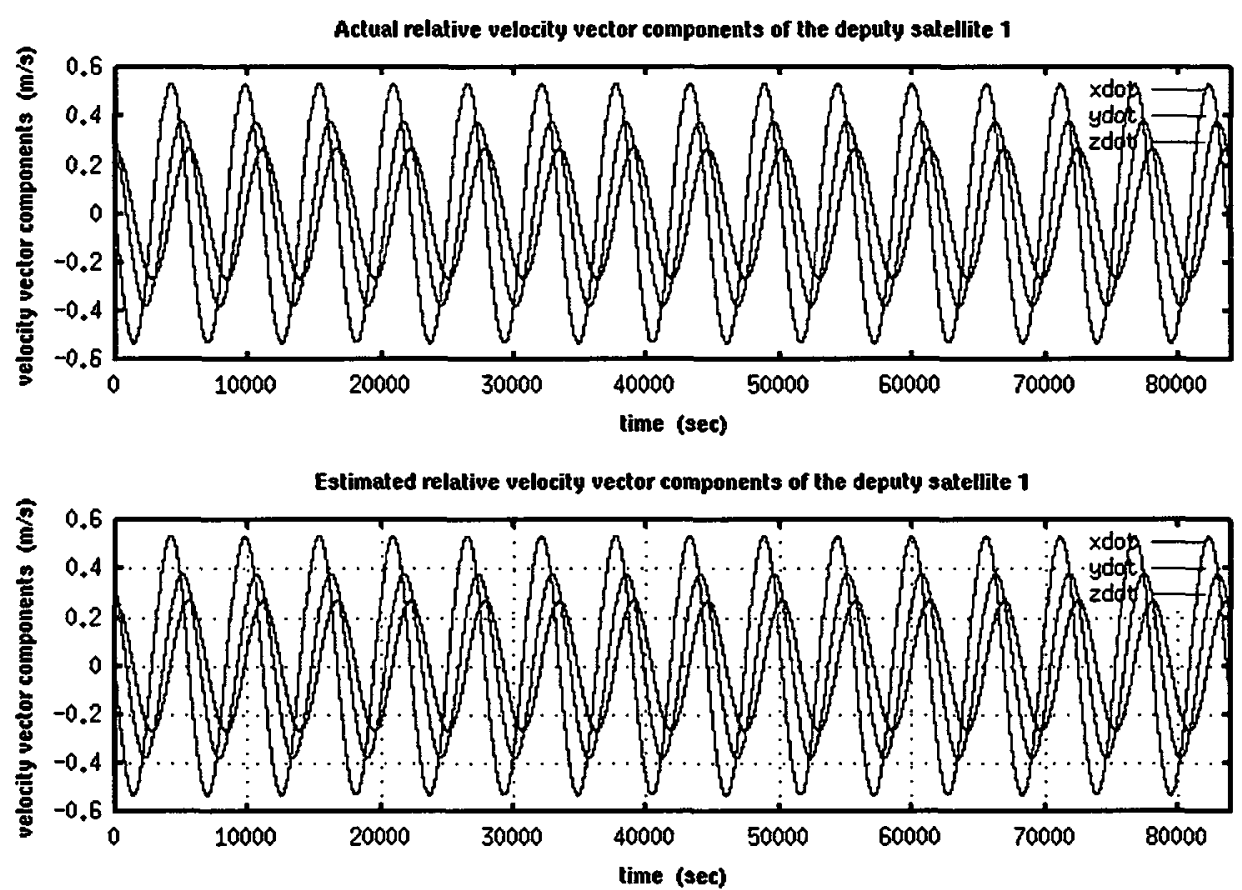

(b) Actual and estimated relative velocity vectors components of the deputy satellite 1

Figure 3.14: Illustration of the deputy satellite 1 actual/estimated relative position and velocity vectors components in the test case 2 


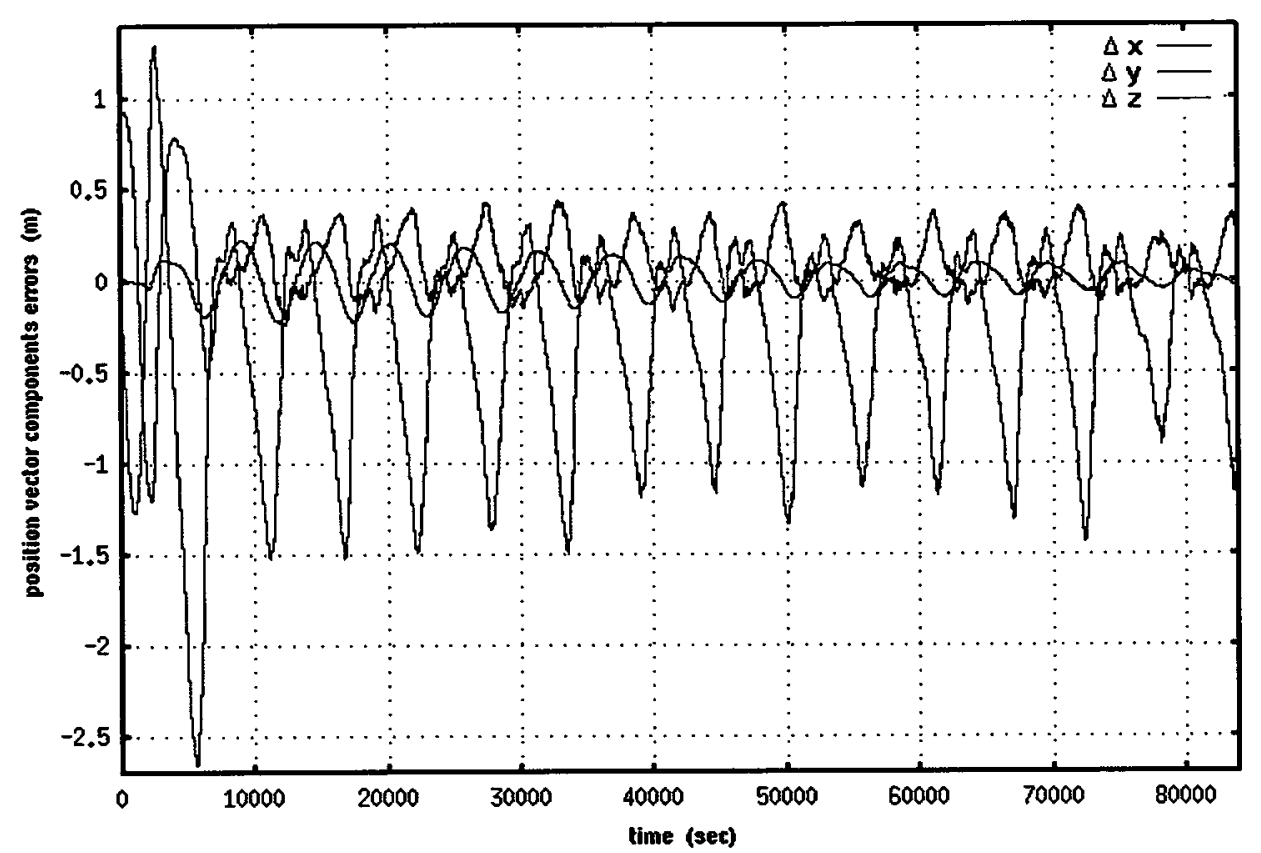

(a) Relative position vector components estimation errors of the deputy satellite 1

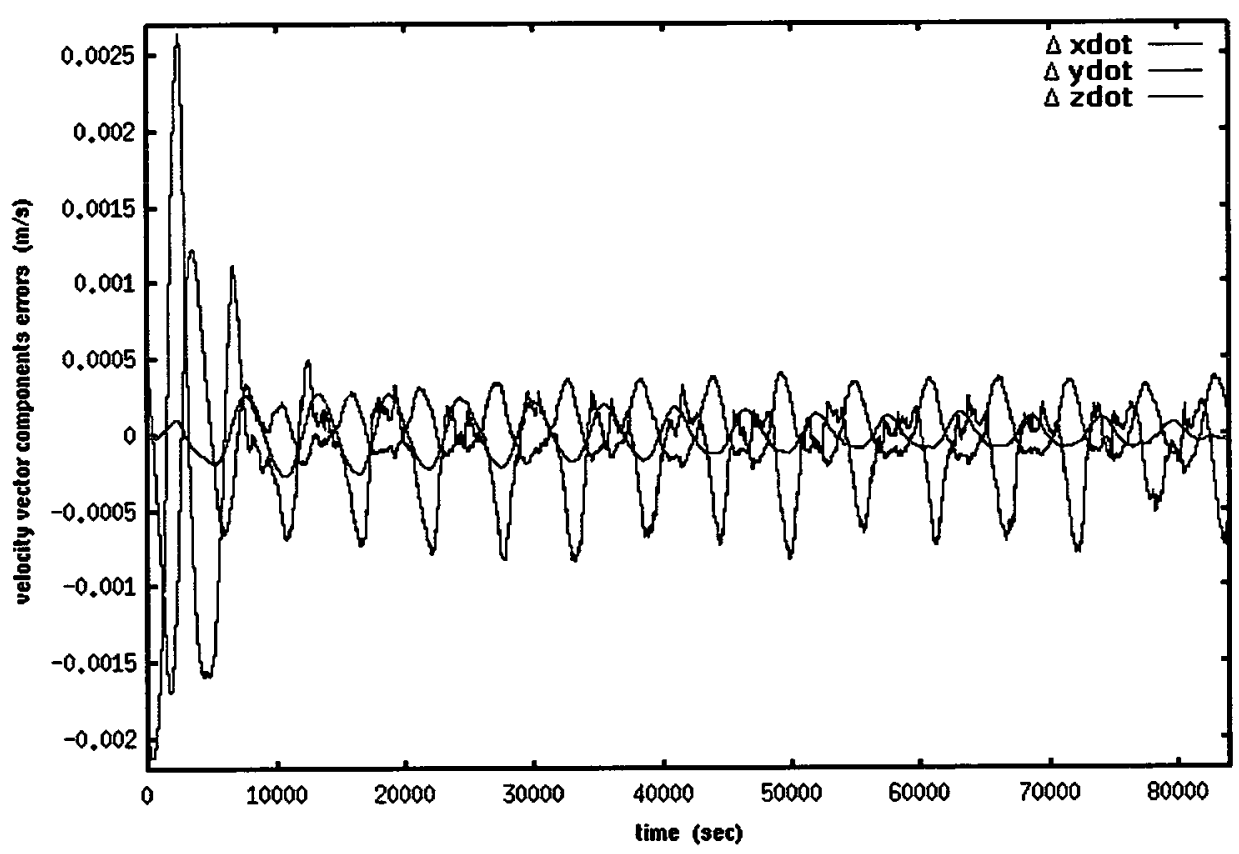

(b) Relative velocity vector components estimation errors of the deputy satellite 1

Figure 3.15: Illustration of the deputy satellite 1 relative position and velocity estimation errors in the test case 2 


\section{Simulation results for the deputy satellite 2}

Figure 3.16 shows the time history of the deputy satellite 2 noisy observations collected by the chief satellite radar. Figure 3.16a displays the slant range vector $\rho$ distance and Figure 3.16b exhibits the azimuth $a z$ and elevation $e l$ angles. The slant range vector $\rho$ time history clearly shows a secular growth in the distance between the chief satellite and the deputy satellite 2. Figure 3.17 displays the deputy satellite 2 actual relative position and velocity vector components in the chief LVLH curvilinear frame and compare it against the estimated relative position and velocity vectors components that are computed by our iterated extended Kalman filter. Figure 3.18 demonstrates the performance of our proposed IEKF in accomplishing the relative orbit determination task for the deputy satellite 2. Figure 3.18a shows that the maximum absolute value of the IEKF estimation errors for the deputy satellite 2 relative position vector components is less than 1.7 meters for the entire simulated flight times. We also notice that there is no indication of secular growth in the estimation errors for the radial, and cross-track relative position vector components. There exists however, a few small secular increases in estimation errors for the intrack element specially on orbits 11 and 14. If we compare Figure 3.18a on page 125 with Figure 2.15a on page 78 , we clearly see that the progressively increasing in-track errors do no exist and after the first orbit, all relative position vector components are confined within a band of -1 to 0.6 meters. Turning our attention to Figure $3.18 \mathrm{~b}$, we note that the maximum absolute value of the IEKF estimation errors for the deputy satellite 2 relative velocity vector components is less 3.7 millimeters per second and there is no sign of secular growth in the estimation errors during 23.25 hours of simulated flight times. Comparing Figure 3.18b on page 125 with Figure $2.15 \mathrm{~b}$ on page 78 easily displays that after the first one and half orbits, the proposed IEKF has limited the relative velocity vector components errors to a band of \pm 0.9 millimeters per second. 
We infer from these test case 2 simulation results that the proposed IEKF performs fairly good in estimating the deputy satellite 2 relative position and velocity vectors.

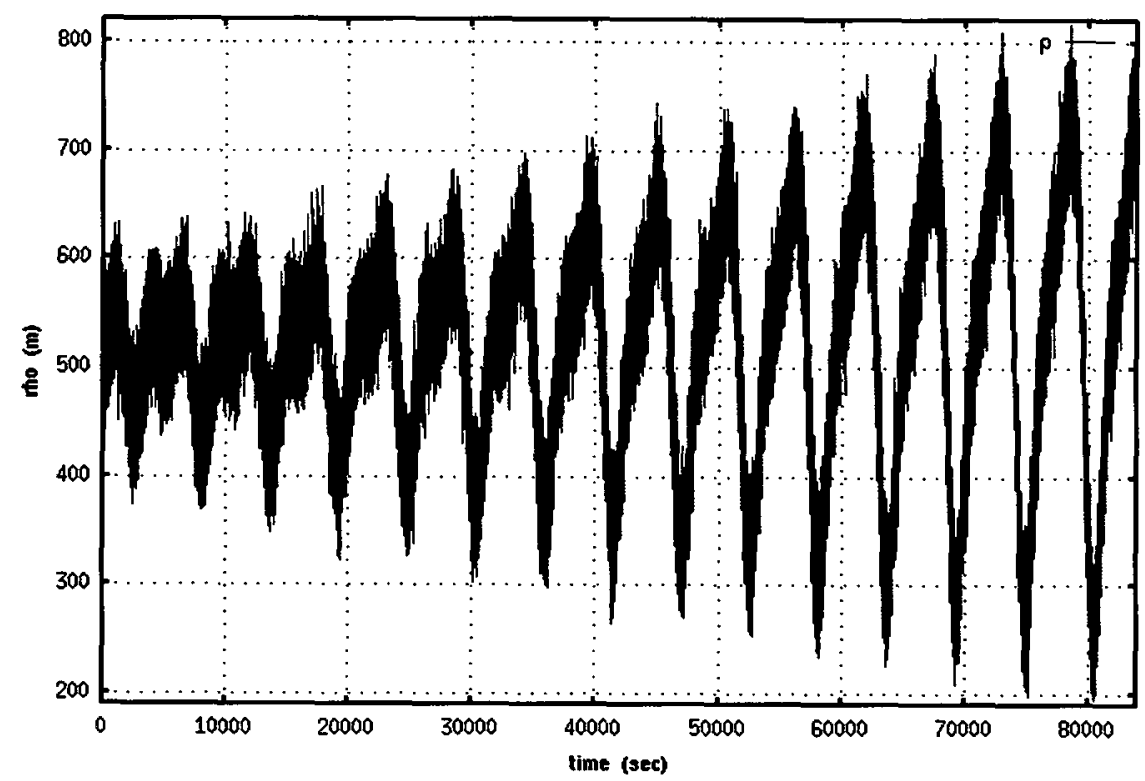

(a) Slant range vector $\rho$ observation of the deputy satellite 2

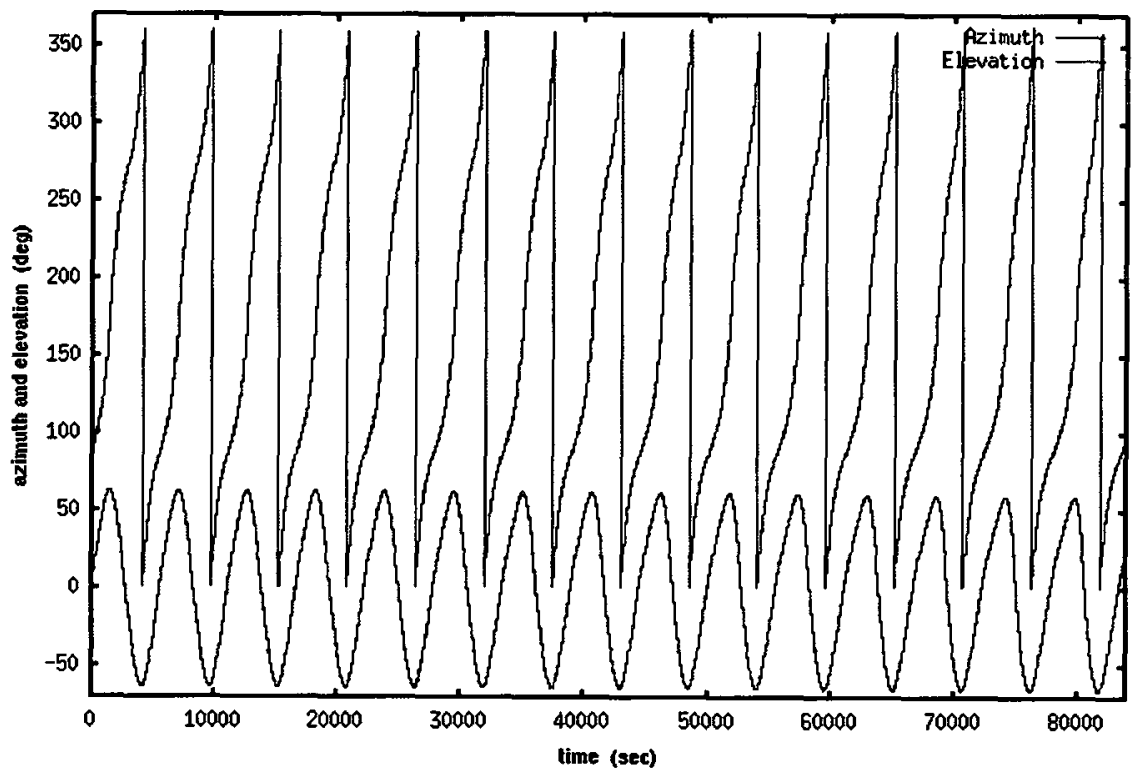

(b) Slant range vector $a z$ and $e l$ observations of the deputy satellite 2

Figure 3.16: Illustration of the deputy satellite 2 slant range vector observations data in the test case 2 

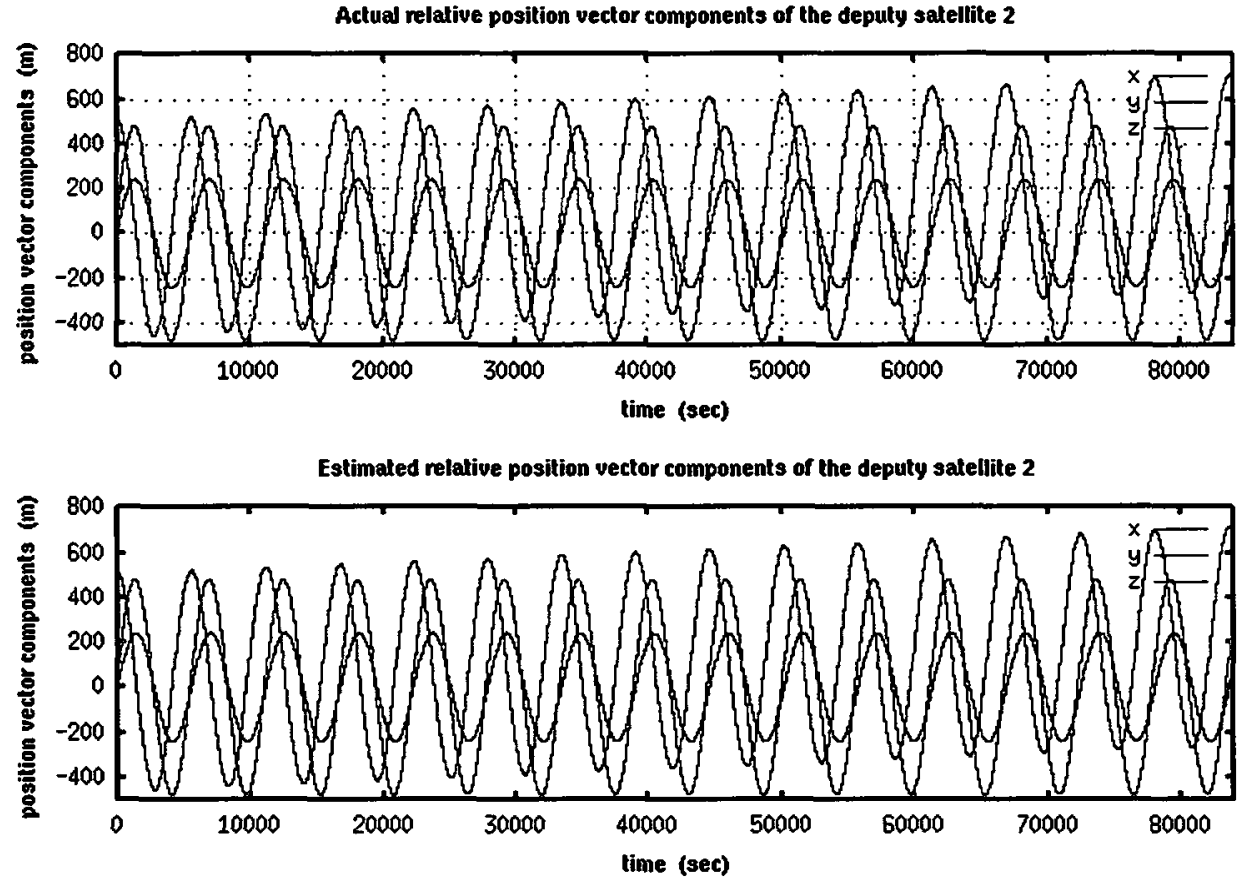

(a) Actual and estimated relative position vectors components of the deputy satellite 2
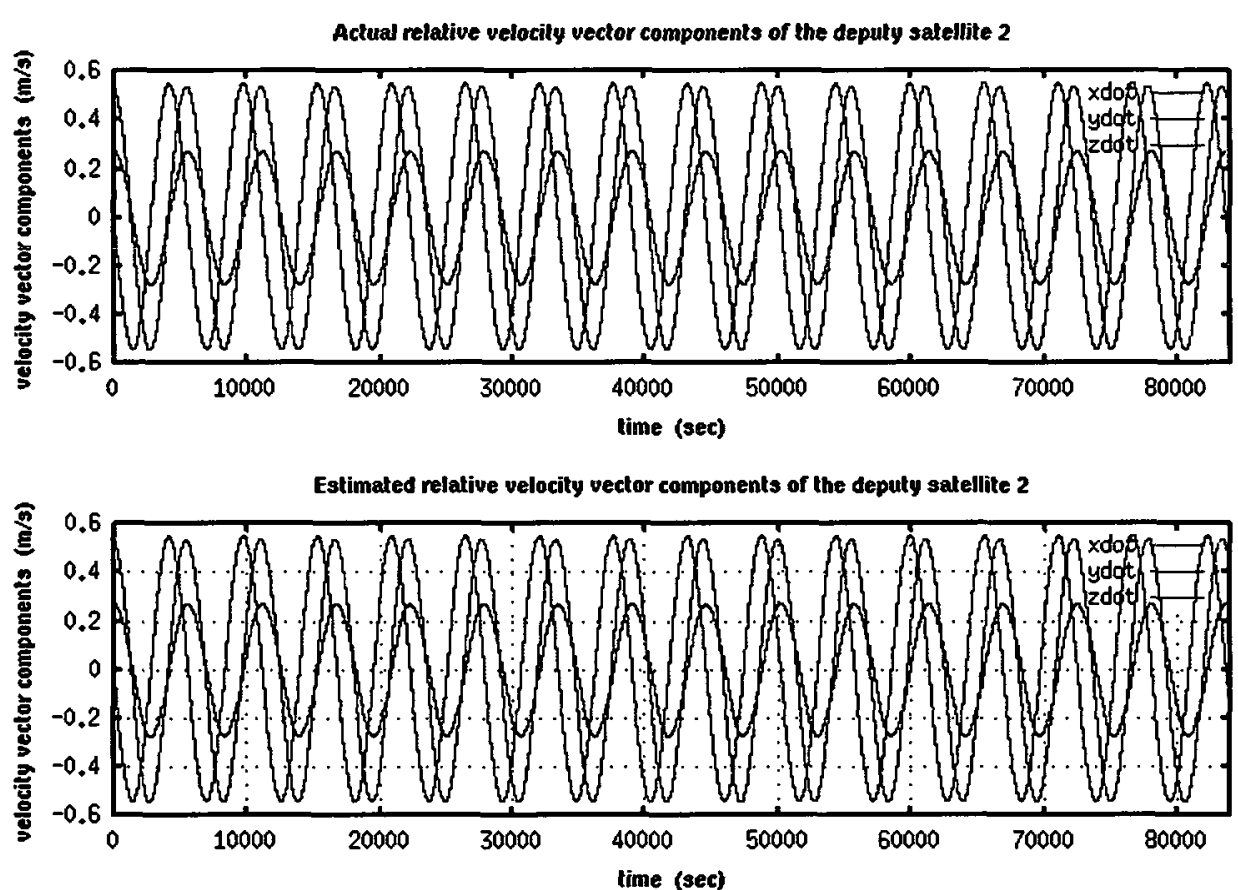

(b) Actual and estimated relative velocity vectors components of the deputy satellite 2

Figure 3.17: Illustration of the deputy satellite 2 actual/estimated relative position and velocity vectors components in the test case 2 


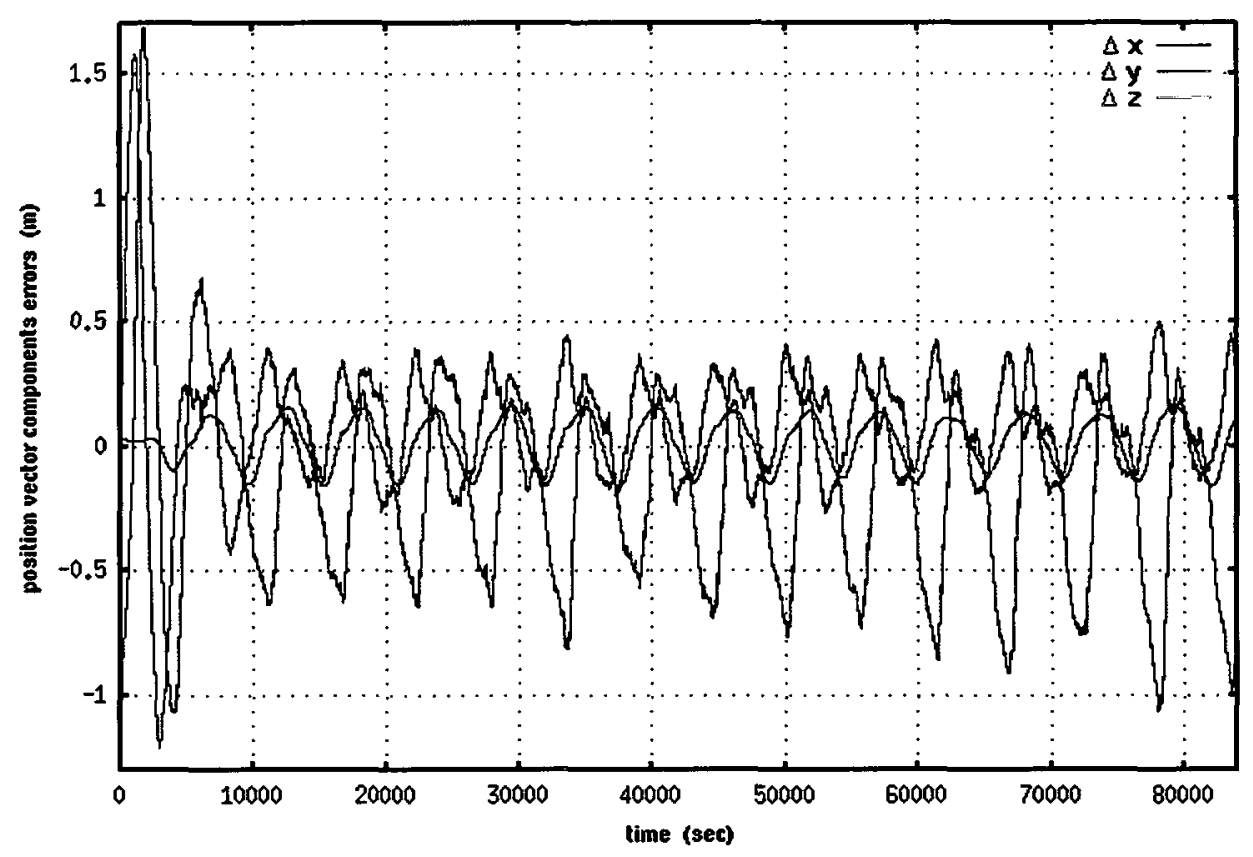

(a) Relative position vector components estimation errors of the deputy satellite 2

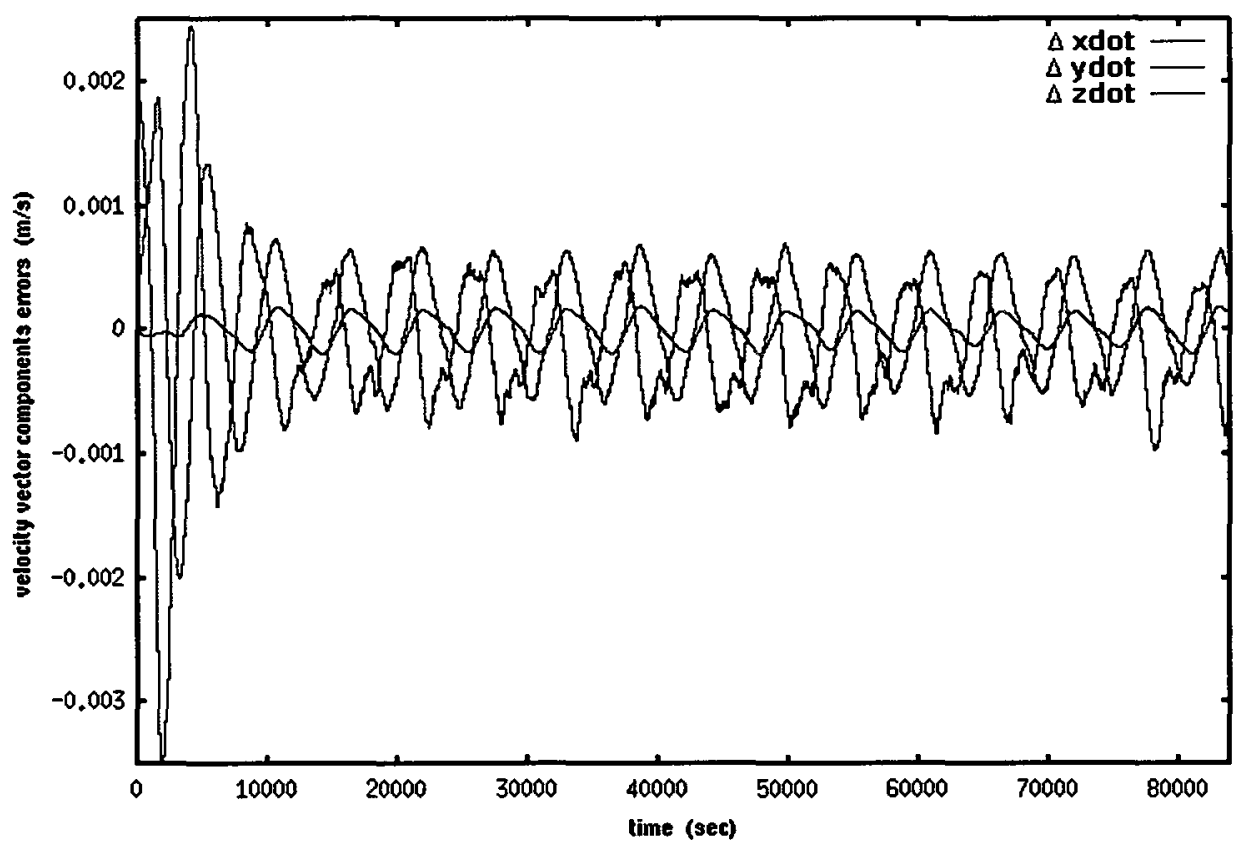

(b) Relative velocity vector components estimation errors of the deputy satellite 2

Figure 3.18: Illustration of the deputy satellite 2 relative position and velocity estimation errors in the test case 2 


\subsubsection{Deputy Satellites Relative Orbit Determinations for Test Case 3}

The initial conditions for the chief and two deputy satellites in this test case were given in tables 2.5 and 2.6. The test case 3 simulation is run for a duration of 15 chief/deputy orbital periods which is equal to 116985 seconds or almost 32.49 hours. Figures 3.19 and 3.20 display the difference between the rectilinear and the curvilinear relative position vector components of the deputy satellites 1 and 2 respectively. It is clear from Figure 3.19 that the maximum absolute value of disparity belongs to the radial component of the relative position vector of deputy satellite 1 and it is almost 34 meters. Similarly in Figure 3.20 for the deputy satellite 2, the maximum absolute value of discrepancy belongs to the radial component of the relative position vector and it is about 43 meters during 32.49 hours of the simulated flight times which is very significant. Therefore approximation (3.4) does not hold during test case 3 simulation and we expect utilizing the deputy satellite slant range vector components $\left(\rho_{x}, \rho_{y}, \rho_{z}\right)^{T}$ in place of curvilinear position vector components $(x, y, z)^{T}$ will add more errors to our IEKF estimated states. 


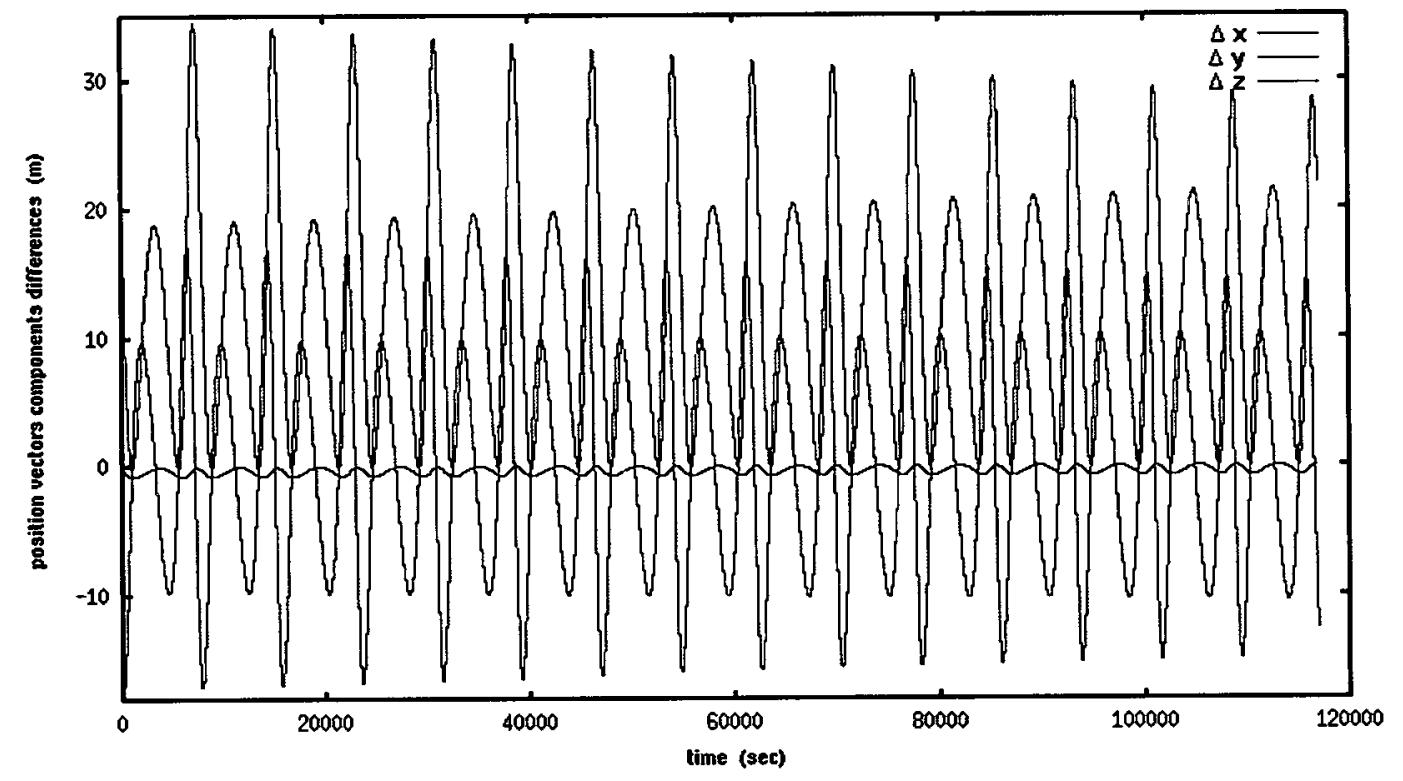

Figure 3.19: Illustration of the deputy satellite 1 rectilinear versus curvilinear relative position vector differences in the test case 3

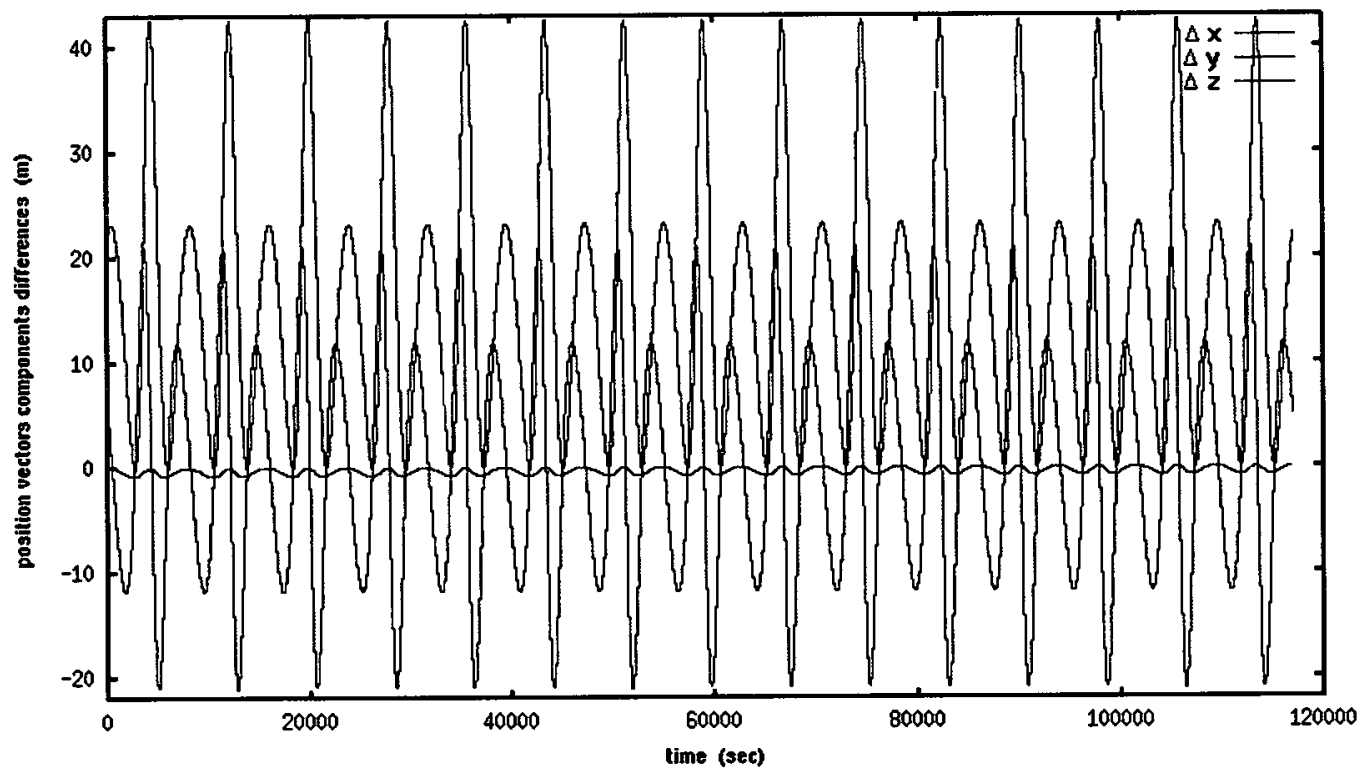

Figure 3.20: Illustration of the deputy satellite 2 rectilinear versus curvilinear relative position vector differences in the test case 3 


\section{Simulation results for the deputy satellite 1}

Figure 3.21 shows the time history of the deputy satellite 1 noisy observations collected by the chief satellite radar. Figure 3.21 a displays the slant range vector $\rho$ distance and Figure 3.21b exhibits the azimuth $a z$ and elevation $e l$ angles. The slant range vector $\rho$ time history subtly shows a secular increase in the relative periapsis distance and a secular decrease in the relative apoapsis distance between the chief satellite and the deputy satellite 1 . Figure 3.22 displays the deputy satellite 1 actual relative position and velocity vector components in the chief LVLH curvilinear frame and compare it against the estimated relative position and velocity vectors components that are computed by our iterated extended Kalman filter. Figure 3.23 demonstrates the performance of our proposed IEKF in accomplishing the relative orbit determination task for the deputy satellite 1. Figure 3.23a shows that the maximum absolute value of the IEKF estimation errors for the deputy satellite 1 relative position vector components is less than 48 meters for the entire simulated flight times. We also notice that there is no indication of secular growth in the estimation errors for the radial, in-track, and cross-track relative position vector components. If we compare Figure $3.23 \mathrm{a}$ on page 131 with Figure 2.18 a on page 85 , we clearly see that the progressively increasing in-track errors do no exist and specially after the first two orbits, all relative position vector components errors are confined within a band of -20 to 25 meters. Turning our attention to Figure $3.23 \mathrm{~b}$, we note that the maximum absolute value of the IEKF estimation errors for the deputy satellite 1 relative velocity vector components is less 10 centimeters per second and similarly there is no sign of secular growth in the estimation errors during 32.49 hours of simulated flight times. Comparing Figure $3.23 \mathrm{~b}$ on page 131 with Figure $2.18 \mathrm{~b}$ on page 85 easily displays that after the first two orbits, the proposed IEKF has limited the relative velocity vector components errors to a band between -4 and 3 centimeters per second.

We infer from these test case 3 simulation results that the proposed IEKF performs 
fairly good in estimating the deputy satellite 1 relative position and velocity vectors.

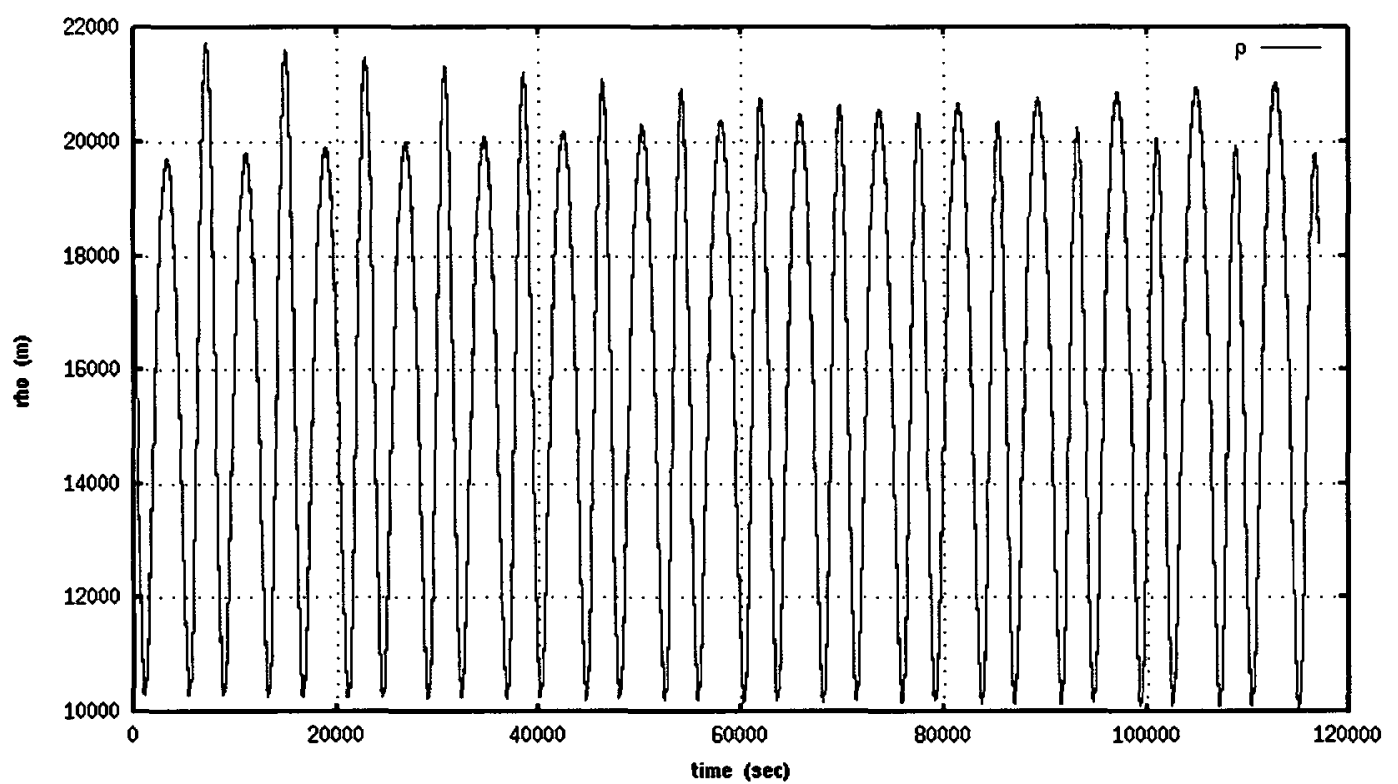

(a) Slant range vector $\rho$ observation of the deputy satellite 1

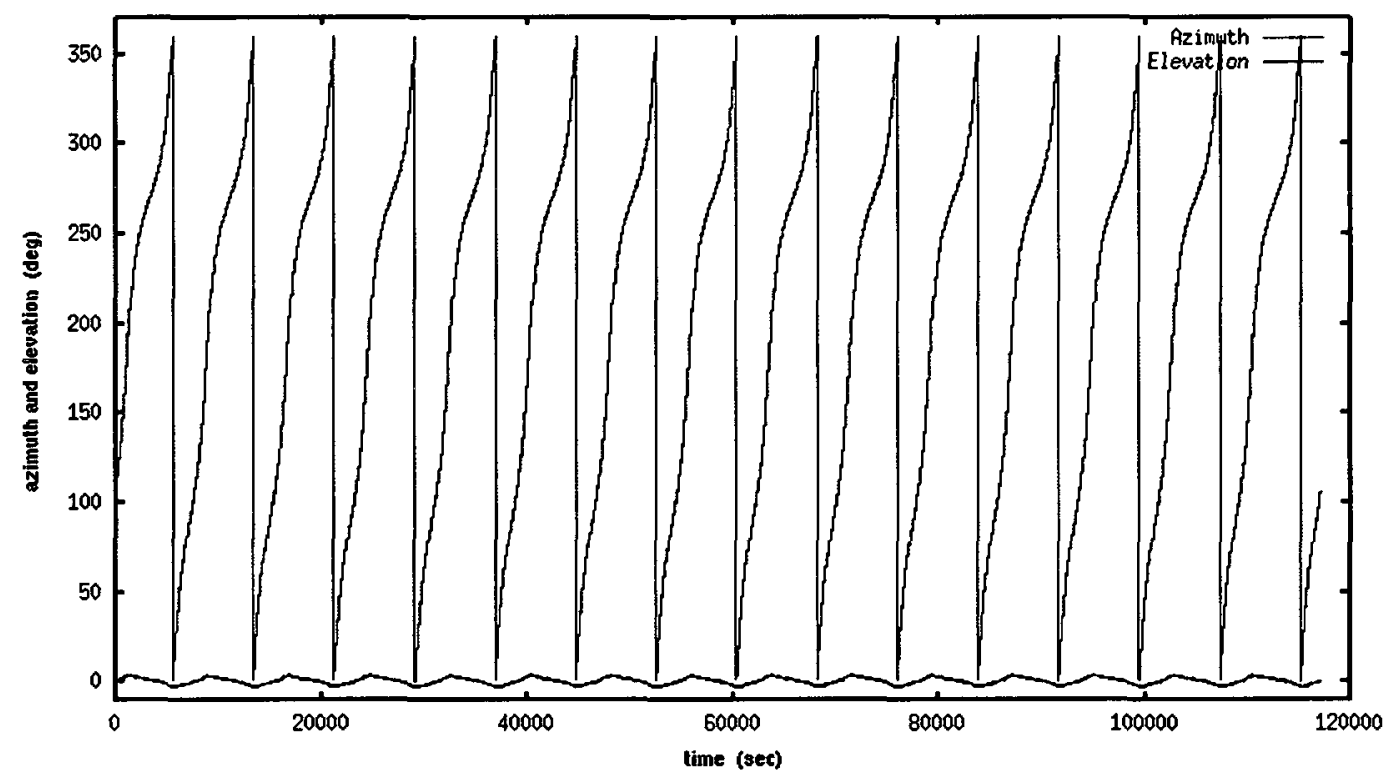

(b) Slant range vector $a z$ and $e l$ observations of the deputy satellite 1

Figure 3.21: Illustration of the deputy satellite 1 slant range vector observations data in the test case 3 

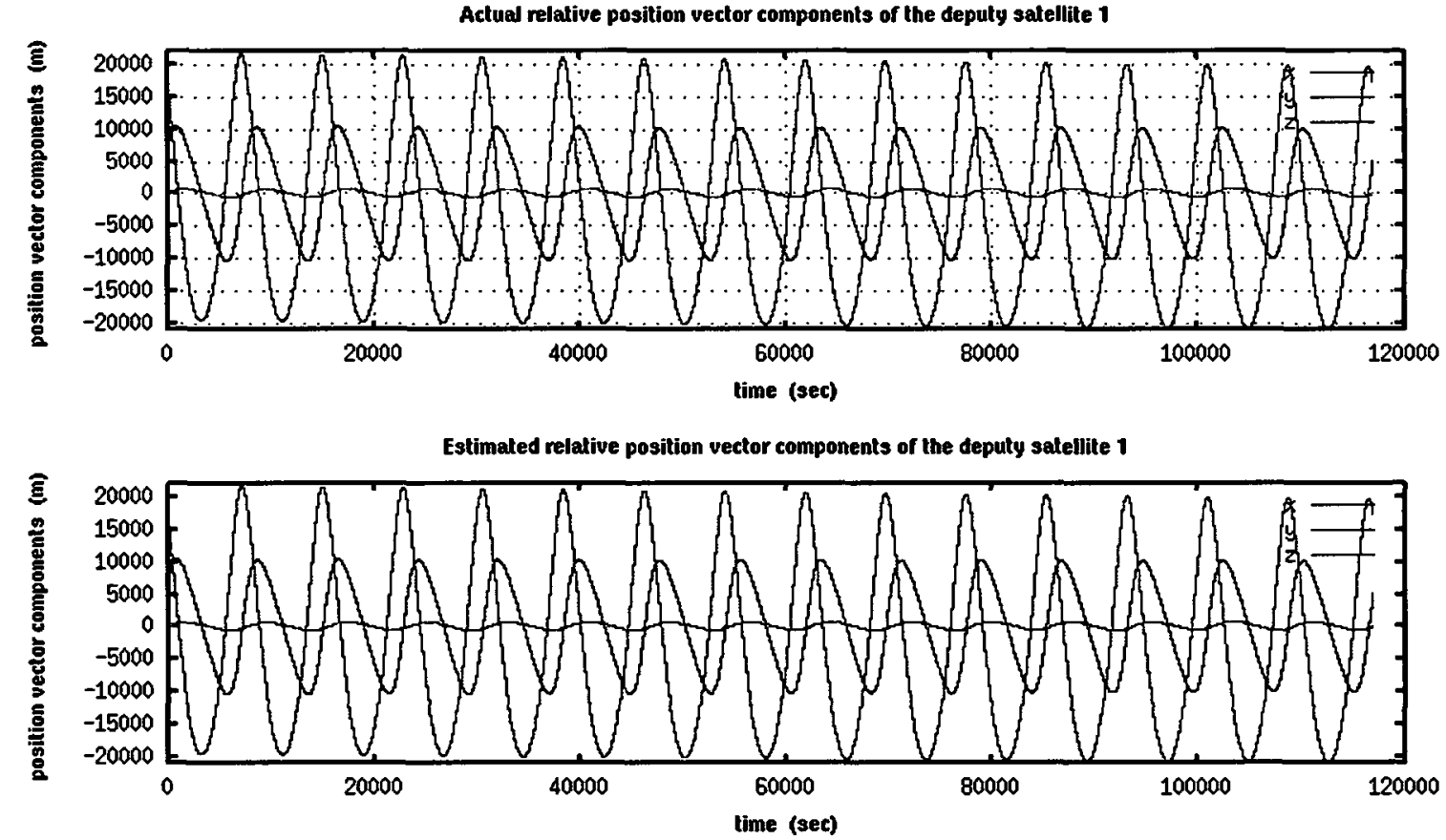

(a) Actual and estimated relative position vectors components of the deputy satellite 1
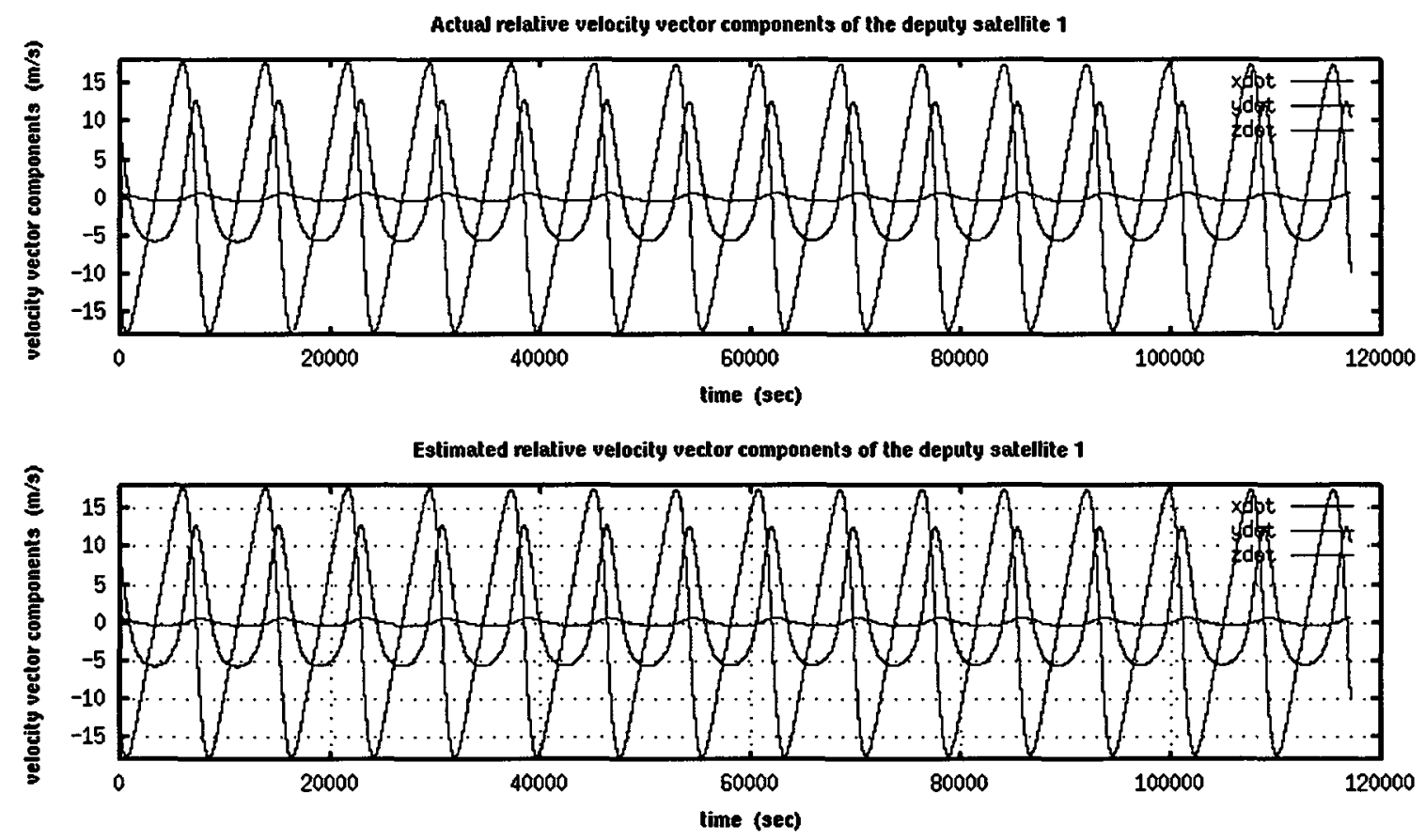

(b) Actual and estimated relative velocity vectors components of the deputy satellite 1

Figure 3.22: Illustration of the deputy satellite 1 actual/estimated relative position and velocity vectors components in the test case 3 


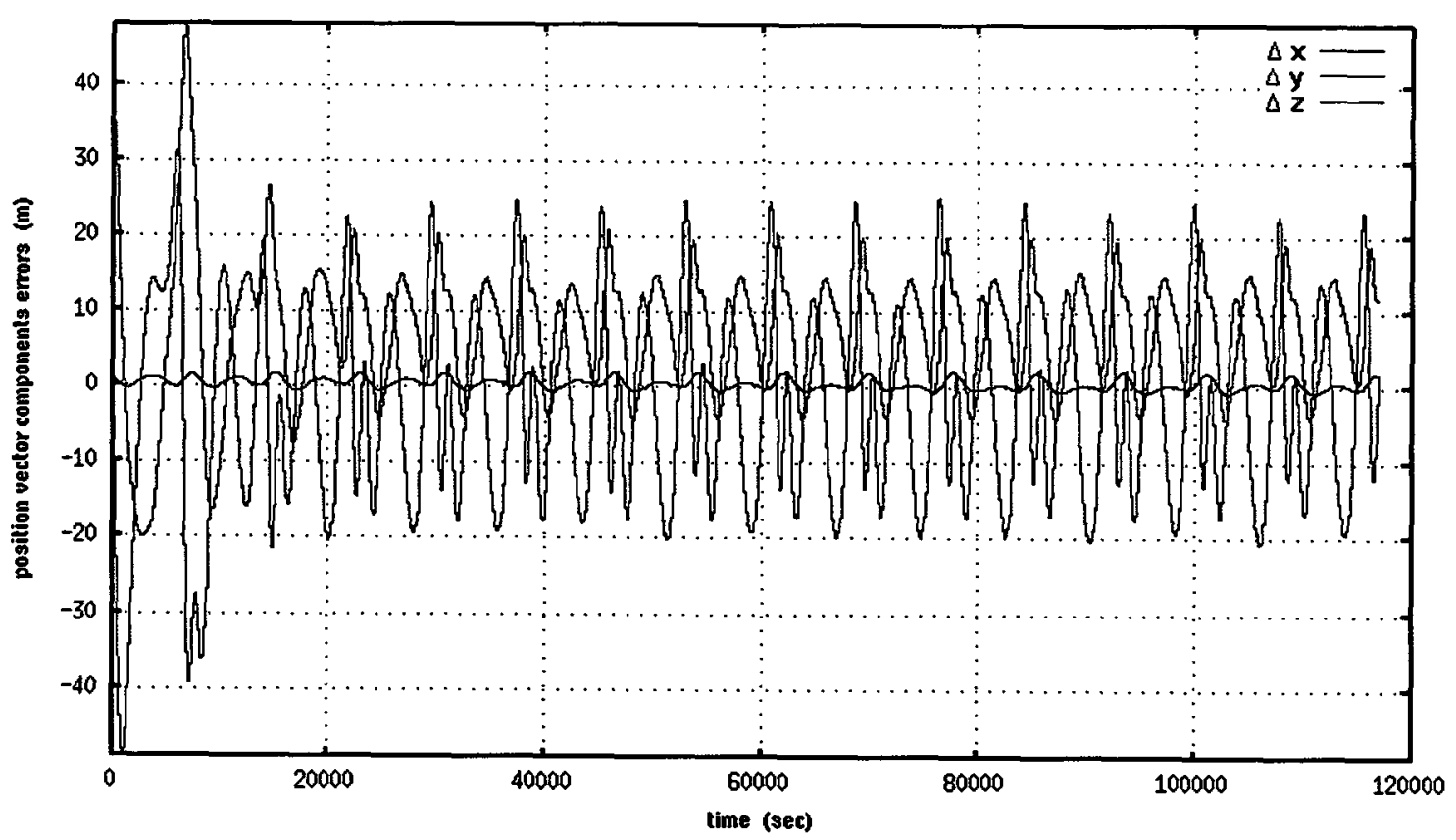

(a) Relative position vector components estimation errors of the deputy satellite 1

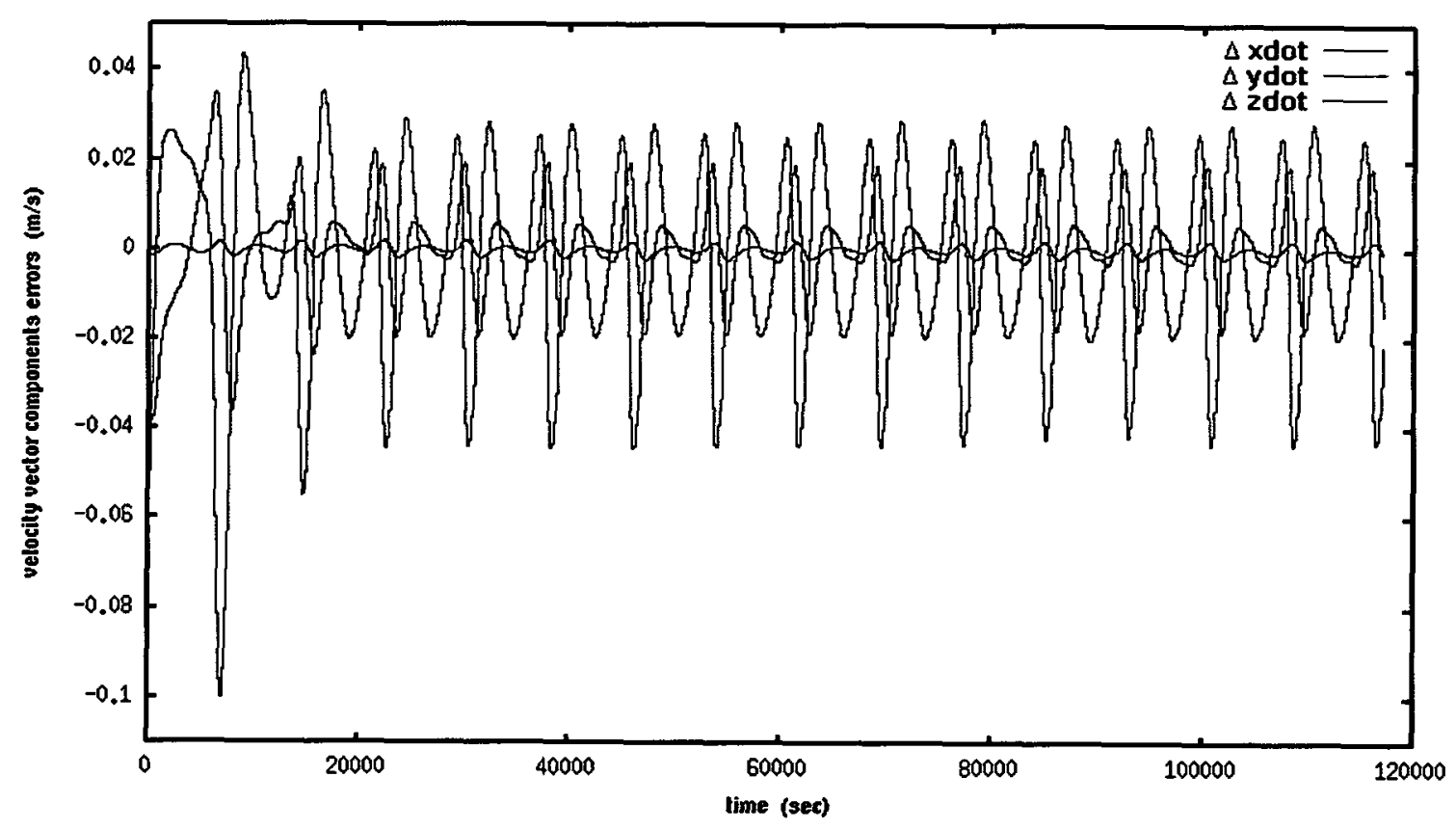

(b) Relative velocity vector components estimation errors of the deputy satellite 1

Figure 3.23: Illustration of the deputy satellite 1 relative position and velocity estimation errors in the test case 3 


\section{Simulation results for the deputy satellite 2}

Figure 3.24 shows the time history of the deputy satellite 2 noisy observations collected by the chief satellite radar. Figure $3.24 \mathrm{a}$ displays the slant range vector $\rho$ distance and Figure 3.24b exhibits the azimuth $a z$ and elevation $e l$ angles. The slant range vector $\rho$ time history shows no secular changes in the relative apoapsis and periapsis distances between the chief satellite and the deputy satellite 2. Figure 3.25 displays the deputy satellite 2 actual relative position and velocity vector components in the chief LVLH curvilinear frame and compare it against the estimated relative position and velocity vectors components that are computed by our iterated extended Kalman filter. Figure 3.26 demonstrates the performance of our proposed IEKF in accomplishing the relative orbit determination task for the deputy satellite 2. Figure 3.26a shows that the maximum absolute value of the IEKF estimation errors for the deputy satellite 2 relative position vector components is less than 130 meters for the entire simulated flight times. We also notice that there is no indication of secular growth in the estimation errors for the radial, in-track, and cross-track relative position vector components. If we compare Figure $3.26 \mathrm{a}$ on page 135 with Figure 2.20a on page 89 , we clearly see that after the first two orbits, all relative position vector components errors are confined within a band between -55 to 35 meters. Turning our attention to Figure $3.26 \mathrm{~b}$, we note that the maximum absolute value of the IEKF estimation errors for the deputy satellite 2 relative velocity vector components is less 19 centimeters per second during 24.81 hours of simulated flight times. Comparing Figure $3.26 \mathrm{~b}$ on page 135 with Figure $2.20 \mathrm{~b}$ on page 89 easily displays that after the first two orbits, the proposed IEKF has limited the relative velocity vector components errors to a band between -7.5 and 5 centimeters per second.

We infer from these test case 3 simulation results that the proposed IEKF performs fairly acceptable in estimating the deputy satellite 2 relative position and velocity vectors. 


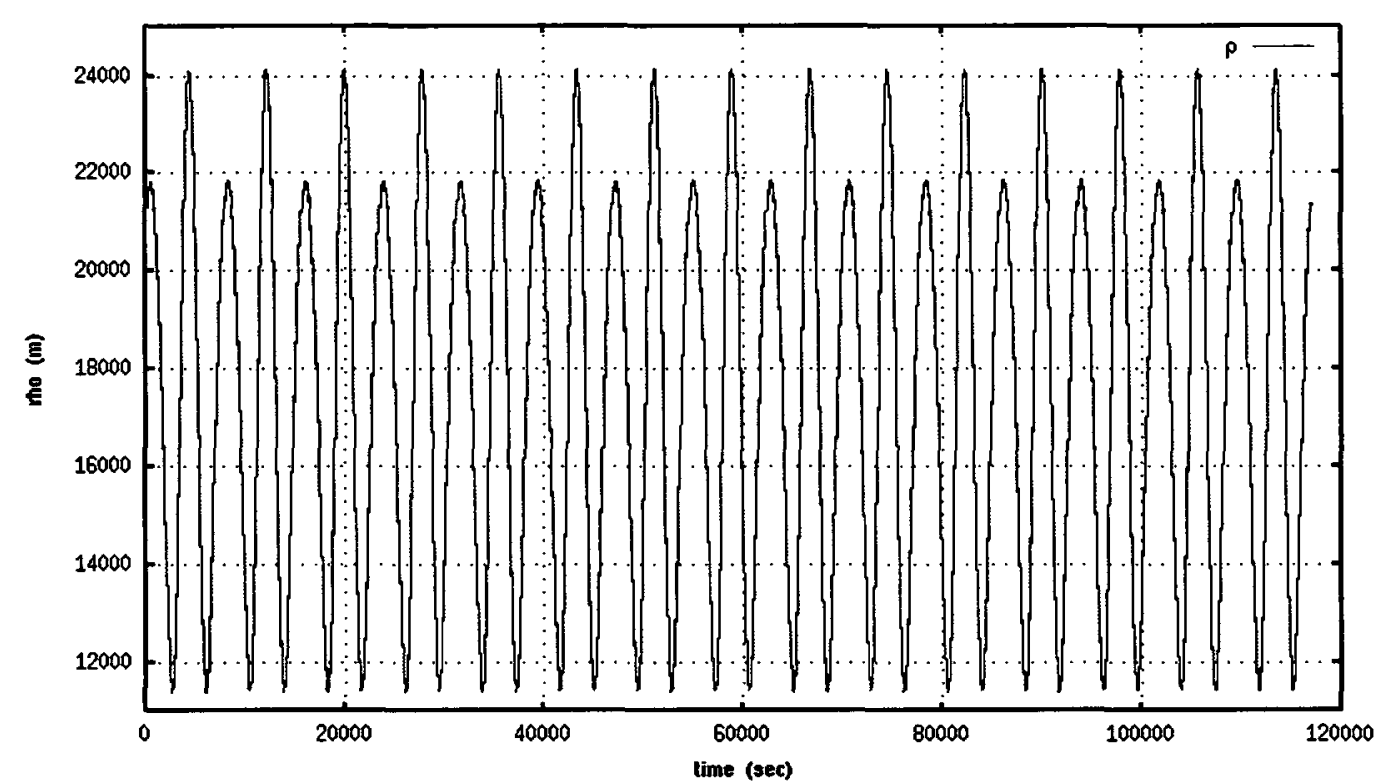

(a) Slant range vector $\rho$ observation of the deputy satellite 2

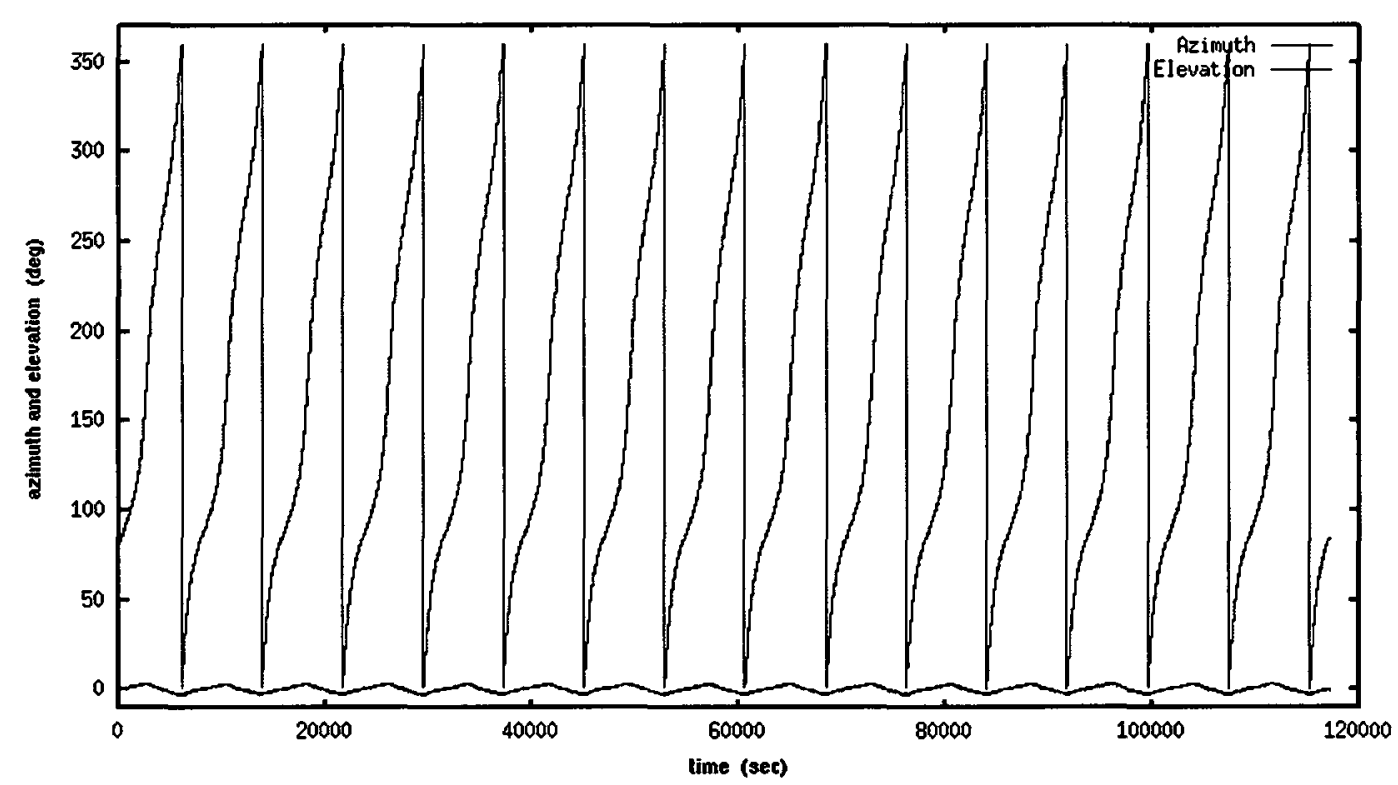

(b) Slant range vector $a z$ and $e l$ observations of the deputy satellite 2

Figure 3.24: Illustration of the deputy satellite 2 slant range vector observations data in the test case 3 

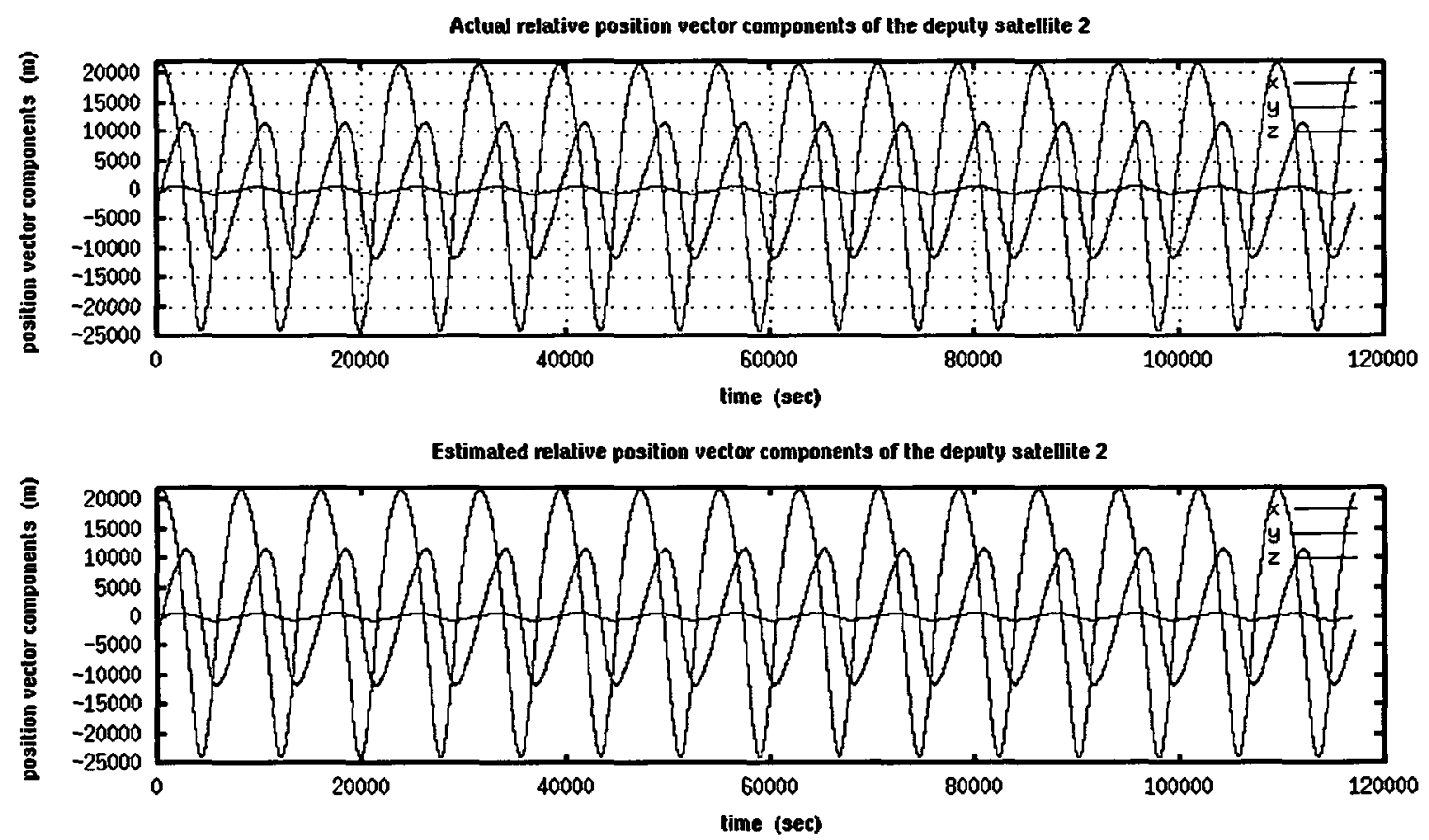

(a) Actual and estimated relative position vectors components of the deputy satellite 2
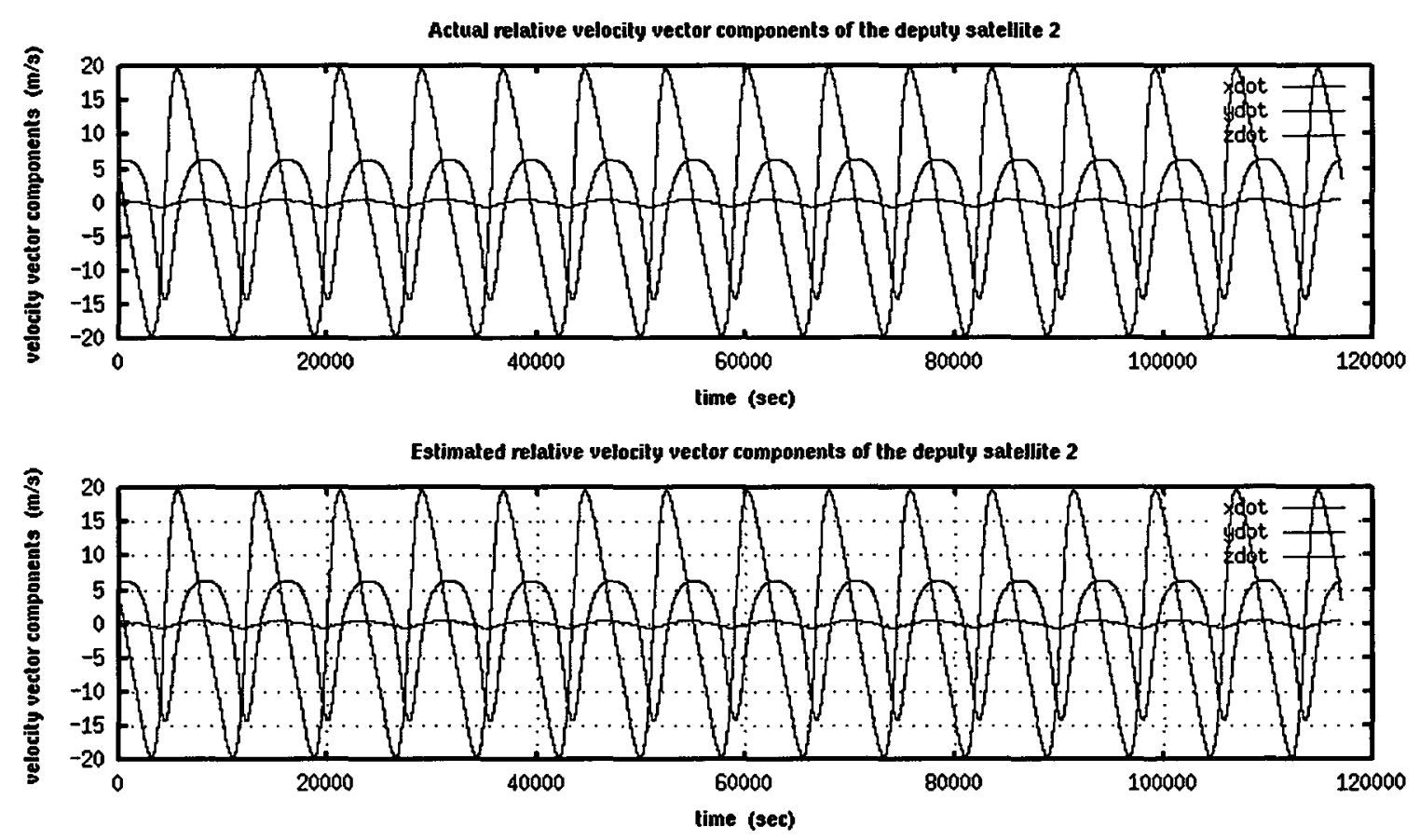

(b) Actual and estimated relative velocity vectors components of the deputy satellite 2

Figure 3.25: Illustration of the deputy satellite 2 actual/estimated relative position and velocity vectors components in the test case 3 


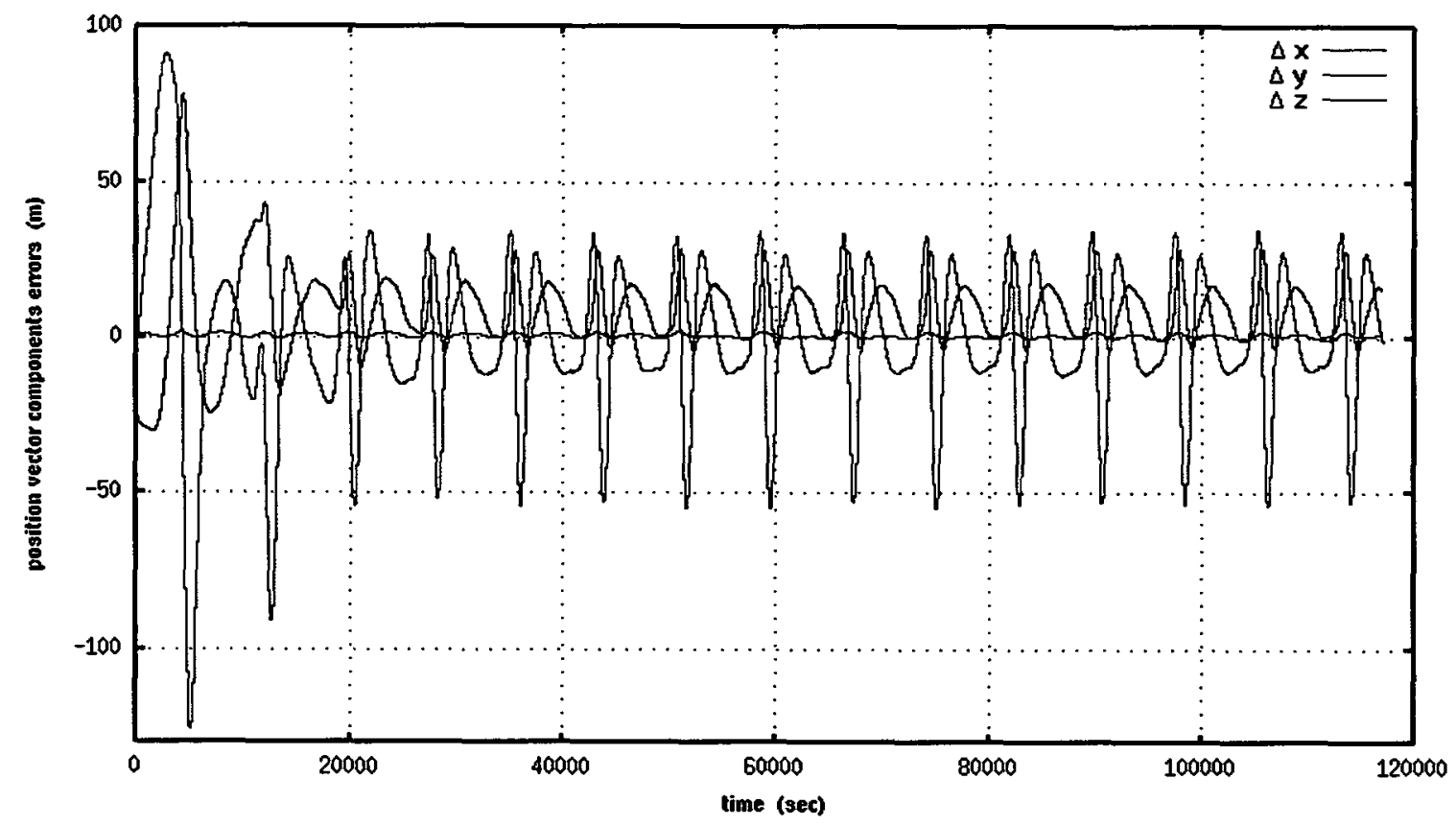

(a) Relative position vector components estimation errors of the deputy satellite 2

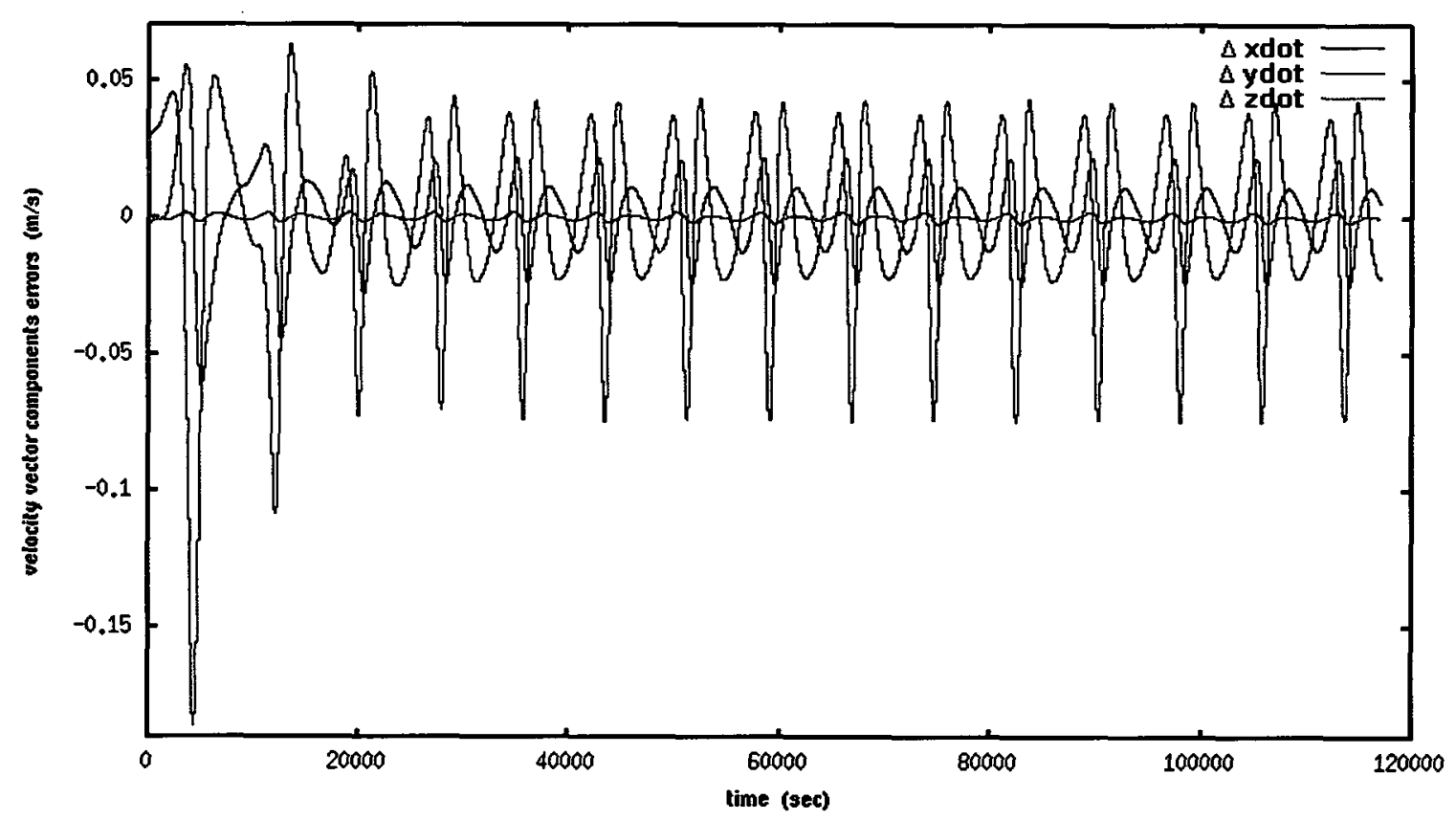

(b) Relative velocity vector components estimation errors of the deputy satellite 2

Figure 3.26: Illustration of the deputy satellite 2 relative position and velocity estimation errors in the test case 3 


\section{Chapter 4}

\section{Summary of Simulation Results, Conclusions and Future Researches}

\subsection{Summary of Simulation Results}

We devised three different simulation test cases for first evaluating the precision of Gim-Alfriend closed-form solution and further to investigate the performance of our proposed relative orbit determination method. In each test case the relative motions of two deputy satellites with respect to a chief satellite were examined. In test cases 1 and 2, a chief and two deputy satellites were flying in close formation and in test case 3, a far formation flight of satellites was studied. We ran each test case simulation for a duration of 15 associated chief orbit periods. The maximum range distances between the chief and any of the two deputy satellites in test cases 1 and 2 were less than 800 and 880 meters respectively. For the test case 3 however, the maximum range distance between the chief and deputy satellites 1 and 2 were about 22 and 24 kilometers.

To analyze the performance of Gim-Alfriend closed-form solution, we compared each deputy satellite actual relative position and velocity vectors components in the chief LVLH curvilinear frame with the predicted relative position and velocity vectors components that were produced by the Gim-Alfriend method in chapter 2 . We observed that for close formation flights of satellites in test cases 1 and 2 , the maximum 
absolute values of prediction errors for the deputy satellites relative position vectors components belong to in-track elements and they are less than 4 meters during the entire simulated flight times. We also noticed that there are often prominent secular growths in the prediction errors for the in-track relative position vector components. We explained that those conspicuous in-track secular growth have mainly resulted from the neglected higher order terms of $J_{2}$ gravitational perturbation in the GimAlfriend state transition matrix. Having turned our attention to the relative velocity vectors components in the test cases 1 and 2 simulation results, we noted that the maximum absolute value of prediction errors for any of the deputy satellites is less than 3 millimeters per second. There are also indications of small secular changes in the prediction errors of the relative velocity vectors components. For the far formation flight test case (test case 3), simulation results exhibit a dramatic change from the corresponding results in the test cases 1 and 2 . The maximum absolute values of prediction errors in the test case 3 for the deputy satellites 1 and 2 relative position vectors components belong to in-track elements and they are about 180 meters and 120 meters respectively. The prediction errors for the relative position vectors radial elements are also very significant. They reach to 50 and 65 meters for the deputy satellite 1 and 2 respectively. We expounded that those large magnitudes for errors should have been expected because when we increase substantially the size of a relative orbit, the linearization of the deputy satellite position and velocity vectors about the chief satellite motion introduces correspondingly much larger errors into the Gim-Alfriend closed-form solution. Test case 3 simulation results also revealed that the prominent secular growths in the prediction errors for the in-track relative position vectors components are significantly larger than their counterparts in the test cases 1 and 2 . We elucidated that those secular growths have resulted primarily from the neglected higher order terms of $J_{2}$ gravitational perturbation and also from the ignored disturbances caused by the zonal harmonics $J_{3}$ to $J_{6}$ of Earth gravitational 
potential. Test case 3 simulation results moreover showed that the maximum absolute value of prediction errors for the deputy satellites 1 and 2 relative velocity vectors components belong to in-track elements and they are about 11 and 15 centimeters per second respectively. It is clear that these prediction errors for the relative velocity vectors components are considerably bigger than their corresponding errors in the test cases 1 and 2 .

We subsequently utilized the same simulation test cases 1 to 3 , to investigate the performance of our proposed relative orbit determination method in chapter 3 . We assumed the chief satellite in every test case is equipped with an active phased array radar. All chief satellites radars were identical and their measurement errors were described by a three - dimensional zero-mean Gaussian probability density function. We compared each deputy satellite actual relative position and velocity vectors components in the chief LVLH curvilinear frame with the estimated relative position and velocity vectors components that were computed by our proposed iterated extended Kalman filter. We observed that for close formation flight of satellites 1 and 2 in test case 1 , after the first orbit all relative position vectors components errors are confined within a band of -1.1 to 0.75 meters. The proposed iterated extended Kalman filter also limits the relative velocity vectors components errors to \pm 0.75 millimeter per second after the first orbit. Moreover we noted that there are no indications of progressively increasing in-track errors in the test case 1 simulation results. Test case 2 simulation results similarly displayed that for close formation flying satellites 1 and 2 , our proposed relative orbit determination method after the first two orbits confine the relative position vectors components errors within a band -1.5 to 0.6 meters. The relative velocity vectors components errors are also limited to a band of \pm 0.9 millimeter per second after the first two orbits. For the satellite 1 in the test case 2, we observe absolutely no secular growth in the estimation errors for the radial, in-track and cross-track relative position and velocity vectors components. For the 
satellite 2 in the test case 2 however, there are a few very small secular increases in estimation errors for the relative position vector in-track element. Finally the test case 3 simulation results reveal that for a far formation flying satellites 1 and 2 , our proposed iterated extended Kalman filter restricts all relative position vectors components errors to a band between -55 to 35 meters after the first two orbits. The relative velocity vectors components errors are also limited after the first two orbits to a band of -7.5 to 5 centimeters per second. Simulation results for the test case 3 further proves that there is no secular growth in the estimation errors for the relative position and velocity vectors components.

\subsection{Conclusions}

We conclude from all the simulation results presented in chapter 2 that the GimAlfriend analytical solution to the relative motion theory brings forth very good predictions for any deputy satellite relative position and velocity vectors provided that the chief and the deputy satellites fly in close formation. We recall that in a centralized-controlled close formation flight of satellites, the maximum separation distance between a chief and any deputy satellite must be less than a few kilometers. It is expected that the Gim-Alfriend analytical solution will offer very poor predictions for any far formation flight of spacecrafts mainly due to significant modeling errors introduced by the linearization process in the Gim-Alfriend geometric method

We further conclude from all the simulation results presented in chapter 3 that our proposed relative orbit determination method performs very good in estimating any deputy satellite relative position and velocity vectors as long as the chief and the deputy satellites fly in close formation. For a close formation flight of spacecrafts, the Gim-Alfriend relative orbit motion linear model is fairly accurate and also the differences between the rectilinear and curvilinear relative position vectors components 
of the deputy satellites are negligible. We have observed that the iterated extended Kalman filter in our proposed relative orbit determination method can effectively compensate for neglected nonlinearities and ignored gravitational perturbations in the system dynamic model and even in the presence of a radar measurement white Gaussian noises it can rather precisely discover the deputy satellites relative states in real time. Our proposed relative orbit determination method is able to significantly restrict the relative position and velocity vectors components errors to a narrow band and often removes any sign of secular growth in errors. In rare cases of the relative orbit motion however, our suggested relative orbit determination method does exhibit small secular increases in estimation errors for the relative orbit state vector components. These uncommon secular growths have very small increase rate and their impact can be completely eliminated by once in a while remotely resetting the iterated extended Kalman filter from a ground station.

We finally conclude that the relative orbit determination method developed and proposed in this thesis for any centralized-controlled autonomous close formation flying satellites is very efficient and precise. These advanced qualities might indeed earn our proposed method a right to be practically utilized in an actual space mission.

\subsection{Recommendations for Future Researches}

We recommend the following future researches for autonomous formation flying spacecrafts.

1. A shaping filter can be added to the iterated extended Kalman filter in our proposed relative orbit determination method for close formation flying spacecrafts to support colored process and measurement noises.

2. The Yan-Vadali exact analytical nonlinear solution to the relative motion theory can be employed along with an unscented Kalman filter (UKF) to develop 
a highly precise relative orbit determination method for both close and far formation flights of spacecrafts. 


\section{References}

[1] Scharf, D. P., Hadaegh, F. Y., and Ploen, S. R., "A Survey of Spacecraft Formation Flying Guidance and Control (Part I) : Guidance," in Proceedings of the 2003 American Control Conference, vol. 2, pp. 1733 - 1739, 2003.

[2] Scharf, D. P., Hadaegh, F. Y., and Ploen, S. R., "A Survey of Spacecraft Formation Flying Guidance and Control (Part II) : Control," in Proceedings of the 2004 American Control Conference, vol. 4, pp. 2976 - 2985, 2004.

[3] Hill, G., "Researches in the Lunar Theory," American Journal of Mathematics, vol. 1 , pp. $5-26,1878$.

[4] Clohessy, W. H., and Wiltshire, R. S., "Terminal Guidance System for Satellite Rendezvous," Journal of the Aerospace Sciences, vol. 27, no. 9, pp. $653-658$, 1960.

[5] Sabol, C., Burns, R., and McLaughlin, C. A., "Satellite Formation Flying Design and Evolution," Journal of Spacecraft and Rockets, vol. 38, no. 2, pp. 270 - 278, 2001.

[6] Lovell, T. A., and Tragesser, S. G., "Analysis of the Reconfiguration and Maintenance of Close Spacecraft Formations," in 13th AAS/AIAA Space Flight Mechanics Conference, AAS 03-139, (Ponce, Puerto Rico), Feburary 2003. 
[7] Inalhan, G., Tillerson, M., and How, J. P., "Relative Dynamics and Control of Spacecraft Formations in Eccentric Orbits," Journal of Guidance, Control, and Dynamics, vol. 25 , no. 1 , pp. $48-59,2002$.

[8] Schaub, H., "Relative Orbit Geometry Through Classical Orbit Element Differences," Journal of Guidance, Control, and Dynamics, vol. 27, no. 5, pp. 839 848,2004 .

[9] Broucke, R. A., "Solution of the Elliptic Rendezvous Problem with the Time as Independant Variable," Journal of Guidance, Control, and Dynamics, vol. 26, no. 4 , pp. $615-621,2003$.

[10] Carter, T. E., "New Form for the Optimal Rendezvous Equations Near a Keplerian Orbit," Journal of Guidance, Control, and Dynamics, vol. 13, no. 1, pp. 183 $-186,1990$.

[11] Lane, C., and Axelrad, P., "Formation Design in Eccentric Orbits Using Linearized Equations of Relative Motion," Journal of Guidance, Control, and Dynamics, vol. 29 , no. 1 , pp. $146-160,2006$.

[12] Melton, R. G., "Time-Explicit Representation of Relative Motion Between Elliptical Orbits," Journal of Guidance, Control, and Dynamics, vol. 23, no. 4, pp. $604-610,2000$.

[13] Sedwick, R. J., Schweighart, S. A., "High-Fidelity Linearized $J_{2}$ Model for Satellite Formation Flight," Journal of Guidance, Control, and Dynamics, vol. 25, no. 6 , pp. $1073-1080,2002$.

[14] Sedwick, R. J., Schweighart, S. A., "Cross-Track Motion of Satellite Formations in the Presence of $J_{2}$ Disturbances," Journal of Guidance, Control, and Dynamics, vol. 28, no. 4 , pp. $824-826,2005$. 
[15] Kechichian, J. A., "Motion in General Elliptic Orbit with Respect to a Dragging and Precessing Coordinate Frame," Journal of the Astronautical Sciences, vol. 46 , no. 1 , pp. $25-45,1998$.

[16] Gim, D. W., and Alfriend, K. T., "State Transition Matrix of Relative Motion for the Perturbed Non-Circular Reference Orbit," Journal of Guidance, Control, and Dynamics, vol. 26, no. 6, pp. $956-971,2003$.

[17] Brouwer, D., "Solution of the Problem of Artificial Satellite Theory Without Drag," The Astronomical Journal, vol. 64, no. 1274, pp. 378 - 397, 1959.

[18] Yan, H., Sengupta, P., Vadali, S. R., and Alfriend, K. T., "Development of a State Transition Matrix for Relative Motion Using the Unit Sphere Approach," in 14th AAS/AIAA Space Flight Mechanics Conference, AAS 04-163, (Maui, Hawaii), Feburary 2004.

[19] Alfriend, K. T., Yan, H., "Evalution and Comparison of Relative Motion Theories," Journal of Guidance, Control, and Dynamics, vol. 28, no. 2, pp. $254-261$, 2005 .

[20] Schaub, H., Junkins, J. L., Analytical Mechanics of Space Systems, ch. 14. Reston, VA: AIAA, 2003.

[21] Schaub, H., Alfriend, K. T., "Hybrid Cartesian and Orbit Element Feedback Law for Formation Flying Spacecraft," Journal of Guidance, Control, and Dynamics, vol. 25 , no. 2 , pp. $383-393,2002$.

[22] Alfriend, K. T., Schaub, H., and Gim, D. W., "Gravitational Perturbations, Nonlinearity and Circular Orbit Assumption Effect on Formation Flying Control Strategies," Advances in the Astronautical Sciences, vol. 104, pp. 139 - 158, 2000. 
[23] Battin, R. H., An Introduction to the Mathematics and Methods of Astrodynamics, pp. 476 - 504. New York: AIAA, 1987.

[24] Vadali, S. R., Schaub, H., and Alfriend, K. T., "Initial Conditions and FuelOptimal Control for Formation Flying of Satellites," in AIAA Guidance, Navigation, and Control Conference, AIAA-99-4265, (Portland, Oregon), August 1999.

[25] Bate, R., Mueller, D. D., and White, J. E., Fundamentals of Astrodynamics. New York: Dover, 1971.

[26] NASA and NIMA, Earth Geopotential Model (EGM-96), 1996. section D.5.

[27] Merson, R. H., "The Motion of a Satellite in an Axi-symmetric Gravitational Field," Geophysics Journal, vol. 4, pp. 17 - 52, 1961.

[28] Maybeck, P. S., Stochastic Models, Estimation, and Control, vol. 1. New York: Academic Press, 1979.

[29] Maybeck, P. S., Stochastic Models, Estimation, and Control, vol. 2. New York: Academic Press, 1982.

[30] Gelb, A., Applied Optimal Estimation. Cambridge, Massachusetts: The MIT Press, 1974

[31] Jazwinski, A. H., Stochastic Processes and Filtering Theory. New York: Academic Press, 1970.

[32] Crassidis, J. L. and Junkins J. L., Optimal Estimation of Dynamic Systems. New York: Chapman \& Hall/CRC, 2004. 


\section{Appendix A}

\section{Disturbed Two-Body Motions Identities}

In this appendix, we prove the disturbed two-body motions identities (2.94a) to $(2.94 \mathrm{~h})$. For identity (2.94a), we begin with the chief radial distance $r$ expression in terms of eccentric anomaly $E$.

$$
r=a(1-e \cos E)
$$

Taking the partial derivative of equation (A.1) with respect to $e$, we obtain

$$
\frac{\partial r}{\partial e}=-a \cos E+a e \sin E \cdot \frac{\partial E}{\partial e}
$$

To express $\partial E / \partial e$, we take the partial derivative of Kepler's equation $M=E-e \sin E$ with respect to e

$$
\frac{\partial M}{\partial e}=0=\frac{\partial E}{\partial e}-\sin E-e \cos E \cdot \frac{\partial E}{\partial e}
$$

Thus we have

$$
\frac{\partial E}{\partial e}=\frac{\sin E}{1-e \cos E}
$$


Substituting equation (A.4) into equation (A.2) and making use of the following elliptical orbit anomaly relation

$$
\cos f=\frac{\cos E-e}{1-e \cos E}
$$

yields identity (2.94a). To prove identity (2.94b) we write

$$
\frac{\partial}{\partial e}\left(\frac{a}{r}\right)=-\frac{a}{r^{2}} \cdot \frac{\partial r}{\partial e}=-\frac{a}{r^{2}} \cdot(-a \cos f)=\left(\frac{a}{r}\right)^{2} \cos f
$$

To prove identity $(2.94 \mathrm{c}$ ), we use equation (A.1) to rewrite equation (A.5) in the following form

$$
\cos f=\frac{a}{r}(\cos E-e)
$$

Taking the partial derivative of equation (A.7) with respect to $e$, we obtain

$$
-\sin f \cdot \frac{\partial f}{\partial e}=\frac{\partial}{\partial e}\left(\frac{a}{r}\right) \cdot(\cos E-e)+\frac{a}{r} \cdot\left(-\sin E \cdot \frac{\partial E}{\partial e}-1\right)
$$

We use equation (A.1) to rewrite equation (A.4) in the following from

$$
\frac{\partial E}{\partial e}=\frac{a}{r} \sin E
$$

Substituting equations (A.6) and (A.9) into equation (A.8) and making use of the elliptical orbit anomaly relations (A.10) and (A.11)

$$
\begin{aligned}
& \sin E=\frac{\sqrt{1-e^{2}} \sin f}{1+e \cos f} \\
& \cos E=\frac{e+\cos f}{1+e \cos f}
\end{aligned}
$$


yields

$$
-\sin f \cdot \frac{\partial f}{\partial e}=-\left(\frac{a}{r}+\frac{1}{1-e^{2}}\right) \sin ^{2} f
$$

By discarding $-\sin f$ from both sides of the above equation we get identity (2.94c). To develop identity (2.94d), we first take the partial derivative of equation (A.1) with respect to $a$

$$
\frac{\partial r}{\partial a}=\frac{r}{a}+a e \sin E \cdot \frac{\partial E}{\partial a}
$$

We next obtain the following expression for $\partial E / \partial a$ by taking the partial derivative of Kepler's equation $M=M_{0}+\sqrt{\frac{\mu}{a^{3}}}\left(t-t_{0}\right)=E-e \sin E$ with respect to $a$

$$
\frac{\partial E}{\partial a}=-\frac{3 n\left(t-t_{0}\right)}{2 r}
$$

Substituting equation (A.14) into equation (A.13) and making use of the elliptical anomaly relation (A.10) yields identity $(2.94 \mathrm{~d})$. To obtain identity $(2.94 \mathrm{e})$ we write

$$
\frac{\partial}{\partial a}\left(\frac{a}{r}\right)=\frac{r-a \cdot \frac{\partial r}{\partial a}}{r^{2}}=\frac{r-a\left(\frac{r}{a}-\frac{3 n e\left(t-t_{0}\right)}{2 \sqrt{1-e^{2}}} \sin f\right)}{r^{2}}=\frac{3 a n e\left(t-t_{0}\right)}{2 r^{2} \sqrt{1-e^{2}}} \sin f
$$

To prove identity (2.94f), we take the partial derivative of equation (A.7) with respect to $a$

$$
-\sin f \cdot \frac{\partial f}{\partial a}=\frac{\partial}{\partial a}\left(\frac{a}{r}\right) \cdot(\cos E-e)+\frac{a}{r} \cdot\left(-\sin E \cdot \frac{\partial E}{\partial a}\right)
$$


By substituting equations (A.14) and (A.15) into equation (A.16) and making use of the elliptical orbit anomaly relations (A.10) and (A.11) we obtain

$$
-\sin f \cdot \frac{\partial f}{\partial a}=\frac{3 a n\left(t-t_{0}\right) \sqrt{1-e^{2}}}{2 r^{2}} \cdot \sin f
$$

Discarding $-\sin f$ from both sides of the above equation yields identity (2.94f). To develop identity $(2.94 \mathrm{~g})$, we write

$$
\frac{\partial r}{\partial f}=\frac{\partial}{\partial f}\left(\frac{a\left(1-e^{2}\right)}{1+e \cos f}\right)=\frac{a\left(1-e^{2}\right) e \sin f}{(1+e \cos f)^{2}}=\frac{r^{2} e}{a\left(1-e^{2}\right)} \sin f
$$

To prove equation $(2.94 \mathrm{~h})$, we use the chain rule of partial differentiation and write

$$
\frac{\partial f}{\partial M_{0}}=\frac{\partial f}{\partial E} \cdot \frac{\partial E}{\partial M_{0}}
$$

We take partial derivatives of equation (A.5) and Kepler's equation $M=E-e \sin E$ with respect to $E$ and obtain $\partial f / \partial E$ and $\partial E / \partial M_{0}$ respectively as follows

$$
\begin{aligned}
\frac{\partial f}{\partial E} & =\frac{a}{r} \sqrt{1-e^{2}} \\
\frac{\partial E}{\partial M_{0}} & =\frac{a}{r}
\end{aligned}
$$

Substituting equations (A.20) and (A.21) into equation (A.19) yields identity (2.94h). 


\section{Appendix B}

\section{Transformation Matrix $\mathcal{T}$}

The transformation matrix $\mathcal{T} \equiv[A(t)+\sigma B(t)]$ was originally produced by professor Kyle T. Alfriend and his research associate Dr. Dong-Woo Gim from Texas A\&M university. Professor Alfriend and Dr. Gim presented matrix $[A(t)+\sigma B(t)]$ on their renowned 2003 paper $^{[16]}$ which was published in the Journal of Guidance, Control and Dynamics. I meticulously redeveloped this matrix to verify the accuracy of its components expressions. The non-zero elements of my developed transformation matrix $\mathcal{T}$ are as follows. Any major difference in component expressions between Gim-Alfriend produced $[A(t)+\sigma B(t)]$ matrix and my developed $\mathcal{T}$ matrix is highlighted by red color.

$$
\begin{aligned}
& \mathcal{T}_{11}=\frac{r}{a} \\
& \mathcal{T}_{12}=\frac{r V_{r}}{V_{t}} \\
& \mathcal{T}_{14}=-\frac{2 r a q_{1}}{p}-\frac{r^{2} \cos \theta}{p} \\
& \mathcal{T}_{15}=-\frac{2 r a q_{2}}{p}-\frac{r^{2} \sin \theta}{p} \\
& \mathcal{T}_{21}=-\frac{V_{r}}{2 a} \\
& \mathcal{T}_{22}=\sqrt{\frac{\mu}{p}}\left(\frac{p}{r}-1\right) \\
& \mathcal{T}_{24}=\frac{V_{r} a q_{1}}{p}+\sqrt{\frac{\mu}{p}} \sin \theta
\end{aligned}
$$




$$
\begin{aligned}
& \mathcal{T}_{25}=\frac{V_{r} a q_{2}}{p}-\sqrt{\frac{\mu}{p}} \cos \theta \\
& \mathcal{T}_{32}=r \\
& \mathcal{T}_{36}=r \cos i \\
& \mathcal{T}_{41}=-\frac{3 V_{t}}{2 a} \\
& \mathcal{T}_{42}=-V_{r} \\
& \mathcal{T}_{43}=-\sigma \frac{V_{t} \sin i \cos i \sin { }^{2} \theta}{p r} \\
& \mathcal{T}_{44}=\frac{3 V_{t} a q_{1}}{p}+2 \sqrt{\frac{\mu}{p}} \cos \theta \\
& \mathcal{T}_{45}=\frac{3 V_{t} a q_{2}}{p}+2 \sqrt{\frac{\mu}{p}} \sin \theta \\
& \mathcal{T}_{46}=V_{r} \cos i+\sigma \frac{V_{t} \sin { }^{2} i \cos i \sin \theta \cos \theta}{p r} \\
& \mathcal{T}_{53}=r \sin \theta \\
& \mathcal{T}_{56}=-r \sin i \cos \theta \\
& \mathcal{T}_{62}=\sigma \frac{V_{t} \sin i \cos i \sin \theta}{p r} \\
& \mathcal{T}_{63}=V_{r} \sin \theta+V_{t} \cos \theta \\
& \mathcal{T}_{66}=\left(V_{t} \sin \theta-V_{r} \cos \theta\right) \sin i+\sigma \frac{V_{t} \sin i \cos ^{2} i \sin \theta}{p r} \\
&
\end{aligned}
$$

where

$$
\begin{aligned}
\sigma & =3 J_{2} R_{e}^{2} \\
p & =a\left(1-q_{1}^{2}-q_{2}^{2}\right) \\
r & =\frac{p}{1+q_{1} \cos \theta+q_{2} \sin \theta} \\
V_{r} & =\sqrt{\frac{\mu}{p}}\left(q_{1} \sin \theta-q_{2} \cos \theta\right) \\
V_{t} & =\sqrt{\frac{\mu}{p}}\left(1+q_{1} \cos \theta+q_{2} \sin \theta\right)
\end{aligned}
$$




\section{Appendix C}

\section{Analytical Inverse of Transformation Matrix $\mathcal{T}$}

The inverse transformation matrix $\mathcal{T}^{-1} \equiv[A(t)+\sigma B(t)]^{-1}$ was originally produced by professor Kyle T. Alfriend and his research associate Dr. Dong-Woo Gim from Texas A\&M university. Professor Alfriend and Dr. Gim presented matrix $[A(t)+$ $\sigma B(t)]^{-1}$ on their renowned 2003 paper $^{[16]}$ which was published in the Journal of Guidance, Control and Dynamics. I meticulously redeveloped this matrix to verify the accuracy of its components expressions. The non-zero elements of my developed inverse transformation matrix $\mathcal{T}^{-1}$ are as follows. There is no major difference in component expressions between Gim-Alfriend produced $[A(t)+\sigma B(t)]^{-1}$ matrix and my developed $\mathcal{T}^{-1}$ matrix.

$$
\begin{aligned}
& \mathcal{T}_{11}^{-1}=-\frac{2 a}{\mu r}\left[\frac{\mu}{r}(3 a-2 r)-a\left(2 V_{r}^{2}+3 V_{t}^{2}\right)\right] \\
& \mathcal{T}_{12}^{-1}=\frac{2 a^{2} V_{r}}{\mu} \\
& \mathcal{T}_{13}^{-1}=\frac{2 a V_{r}}{\mu}\left[\frac{V_{t}}{p}(2 a-r)-\frac{a}{r V_{t}}\left(V_{r}^{2}+2 V_{t}^{2}\right)\right] \\
& \mathcal{T}_{14}^{-1}=-\frac{2 a r}{\mu}\left[\frac{V_{t}}{p}(2 a-r)-\frac{a}{r V_{t}}\left(V_{r}^{2}+2 V_{t}^{2}\right)\right] \\
& \mathcal{T}_{15}^{-1}=-\sigma\left(\frac{2 a \sin i \cos i \sin \theta}{\mu p r^{2}}\right)\left[\frac{\mu}{r}(2 a-r)-a\left(V_{r}^{2}+2 V_{t}^{2}\right)\right] \\
& \mathcal{T}_{23}^{-1}=\frac{1}{r}+\sigma\left(\frac{\cos ^{2} i \sin ^{2} \theta}{p r^{2}}\right)
\end{aligned}
$$




$$
\begin{aligned}
& \mathcal{T}_{25}^{-1}=\left(\frac{V_{r} \sin \theta+V_{t} \cos \theta}{r V_{t}}\right)\left(\frac{\cos i}{\sin i}\right) \\
& \mathcal{T}_{26}^{-1}=-\left(\frac{\sin \theta \cos i}{V_{t} \sin i}\right) \\
& \mathcal{T}_{33}^{-1}=-\sigma\left(\frac{\sin i \cos i \sin \theta \cos \theta}{p r^{2}}\right) \\
& \mathcal{T}_{35}^{-1}=\frac{V_{t} \sin \theta-V_{r} \cos \theta}{r V_{t}} \\
& \mathcal{T}_{36}^{-1}=\frac{\cos \theta}{V_{t}} \\
& \mathcal{T}_{41}^{-1}=\frac{p\left(2 V_{r} \sin \theta+3 V_{t} \cos \theta\right)}{r^{2} V_{t}} \\
& \mathcal{T}_{42}^{-1}=\sqrt{\frac{p}{\mu}} \sin \theta \\
& \mathcal{T}_{43}^{-1}=-\frac{\left(V_{r}^{2}+V_{t}^{2}\right) \sin \theta+V_{r} V_{t} \cos \theta}{\mu}+\frac{\sin \theta}{r}+ \\
& \sigma\left(\frac{\cos ^{2} i \sin ^{2} \theta}{V_{t}^{2} r^{3}}\right)\left[V_{t}\left(V_{r} \cos \theta-V_{t} \sin \theta\right)+\frac{\mu \sin \theta}{r}\right] \\
& \mathcal{T}_{44}^{-1}=2 \sqrt{\frac{p}{\mu}} \cos \theta+\frac{r V_{r} \sin \theta}{\mu} \\
& \mathcal{T}_{45}^{-1}=\left[\frac{\left(V_{r}^{2}-V_{t}^{2}\right) \sin \theta \cos \theta+V_{r} V_{t}\left(\cos ^{2} \theta-\sin ^{2} \theta\right)}{\mu}\right]\left(\frac{\cos i}{\sin i}\right)+ \\
& {\left[\frac{\sin \theta\left(V_{r} \sin \theta+V_{t} \cos \theta\right)}{r V_{t}}\right]\left(\frac{\cos i}{\sin i}\right)+} \\
& \sigma\left(\frac{\sin i \cos i \sin \theta}{V_{t} r^{3}}\right)\left(V_{t} \sin \theta+2 V_{t} \cos \theta\right) \\
& \mathcal{T}_{46}^{-1}=-\left[\frac{r \sin \theta\left(V_{r} \cos \theta-V_{t} \sin \theta\right)}{\mu}+\frac{\sin ^{2} \theta}{V_{t}}\right]\left(\frac{\cos i}{\sin i}\right) \\
& \mathcal{T}_{51}^{-1}=-\frac{p\left(2 V_{r} \cos \theta-3 V_{t} \sin \theta\right)}{r^{2} V_{t}} \\
& \mathcal{T}_{52}^{-1}=-\sqrt{\frac{p}{\mu}} \cos \theta \\
& \mathcal{T}_{53}^{-1}=\frac{\left(V_{r}^{2}+V_{t}^{2}\right) \cos \theta-V_{r} V_{t} \sin \theta}{\mu}-\frac{\cos \theta}{r}+ \\
& \sigma\left(\frac{\cos ^{2} i \sin ^{2} \theta}{V_{t}^{2} r^{3}}\right)\left[V_{t}\left(V_{r} \sin \theta+V_{t} \cos \theta\right)-\frac{\mu \cos \theta}{r}\right] \\
& \mathcal{T}_{54}^{-1}=2 \sqrt{\frac{p}{\mu}} \sin \theta-\frac{r V_{r} \cos \theta}{\mu}
\end{aligned}
$$




$$
\begin{aligned}
\mathcal{T}_{55}^{-1}= & {\left[\frac{\left(V_{r} \sin \theta+V_{t} \cos \theta\right)^{2}}{\mu}-\frac{\cos \theta\left(V_{r} \sin \theta+V_{t} \cos \theta\right)}{r V_{t}}\right]\left(\frac{\cos i}{\sin i}\right)-} \\
& \sigma\left(\frac{\sin i \cos i \sin \theta}{V_{t} r^{3}}\right)\left(V_{r} \cos \theta-2 V_{t} \sin \theta\right) \\
\mathcal{T}_{56}^{-1}= & -\left[\frac{r \sin \theta\left(V_{r} \sin \theta+V_{t} \cos \theta\right)}{\mu}-\frac{\sin \theta \cos \theta}{V_{t}}\right]\left(\frac{\cos i}{\sin i}\right) \\
\mathcal{T}_{63}^{-1}= & -\sigma\left(\frac{\cos i \sin ^{2} \theta}{p r^{2}}\right) \\
\mathcal{T}_{65}^{-1}= & -\left(\frac{V_{r} \sin \theta+V_{t} \cos \theta}{r V_{t} \sin i}\right) \\
\mathcal{T}_{66}^{-1}= & \frac{\sin \theta}{V_{t} \sin i}
\end{aligned}
$$

where

$$
\begin{aligned}
|\mathcal{T}| & =-\frac{\mu r^{3} V_{t} \sin i}{2 a p} \\
\sigma & =3 J_{2} R_{e}^{2} \\
p & =a\left(1-q_{1}^{2}-q_{2}^{2}\right) \\
r & =\frac{p}{1+q_{1} \cos \theta+q_{2} \sin \theta} \\
V_{r} & =\sqrt{\frac{\mu}{p}}\left(q_{1} \sin \theta-q_{2} \cos \theta\right) \\
V_{t} & =\sqrt{\frac{\mu}{p}}\left(1+q_{1} \cos \theta+q_{2} \sin \theta\right)
\end{aligned}
$$




\section{Appendix D}

\section{Mean State Transition Matrix $\Phi_{\delta \bar{e}}\left(t, t_{0}\right)$}

The mean state transformation matrix $\Phi_{\delta \bar{e}}\left(t, t_{0}\right)$ was originally produced by professor Kyle T. Alfriend and his research associate Dr. Dong-Woo Gim from Texas A\&M university. Professor Alfriend and Dr. Gim presented matrix $\Phi_{\delta \bar{e}}\left(t, t_{0}\right)$ on their renowned 2003 paper $^{[16]}$ which was published in the Journal of Guidance, Control and Dynamics. I utilized Maxima computer algebra system for symbolic manipulation of mathematical expressions and rederived this matrix to verify the accuracy of Gim-Alfriend matrix $\Phi_{\delta \bar{e}}\left(t, t_{0}\right)$ components expressions. The non-zero elements of my developed mean state transformation matrix $\Phi_{\delta \bar{e}}\left(t, t_{0}\right)$ are as follows. There is no major difference in component expressions between Gim-Alfriend produced mean state transition matrix and my derived matrix.

$$
\begin{aligned}
\Phi_{\delta \bar{e}, 11}= & 1 \\
\Phi_{\delta \bar{e}, 21}= & -\frac{\left(t-t_{0}\right)}{G_{\theta}}\left\{\left(\frac{3}{2} \frac{n_{0}}{a_{0}}\right)+\frac{7 \sigma}{8}\left(\frac{n_{0}}{a_{0} p_{0}^{2}}\right) \times\right. \\
& {\left.\left[\eta_{0}\left(3 \cos ^{2} i_{0}-1\right)+K\left(5 \cos ^{2} i_{0}-1\right)\right]\right\} } \\
\Phi_{\delta \bar{e}, 22}= & -\frac{G_{\theta_{0}}}{G_{\theta}} \\
\Phi_{\delta \bar{e}, 23}= & -\frac{\left(t-t_{0}\right)}{G_{\theta}}\left\{\frac{\sigma}{2}\left(\frac{n_{0}}{p_{0}^{2}}\right)\left(\sin i_{0} \cos i_{0}\right)\left(3 \eta_{0}+5 K\right)\right\}
\end{aligned}
$$




$$
\begin{aligned}
& \Phi_{\delta \bar{e}, 24}=-\frac{1}{G_{\theta}}\left\{G_{q_{10}}+G_{q_{1}} \cos \left(\dot{\omega}^{(s)}\left(t-t_{0}\right)\right)+G_{q_{2}} \sin \left(\dot{\omega}^{(s)}\left(t-t_{0}\right)\right)\right\}+ \\
& \frac{\left(t-t_{0}\right)}{G_{\theta}}\left(\frac{\sigma}{4}\right)\left(\frac{a_{0} n_{0} q_{10}}{p_{0}^{3}}\right)\left[3 \eta_{0}\left(3 \cos ^{2} i_{0}-1\right)+4 K\left(5 \cos ^{2} i_{0}-1\right)\right] \\
& \Phi_{\delta \bar{e}, 25}=-\frac{1}{G_{\theta}}\left\{G_{q_{20}}-G_{q_{1}} \sin \left(\dot{\omega}^{(s)}\left(t-t_{0}\right)\right)+G_{q_{2}} \cos \left(\dot{\omega}^{(s)}\left(t-t_{0}\right)\right)\right\}+ \\
& \frac{\left(t-t_{0}\right)}{G_{\theta}}\left(\frac{\sigma}{4}\right)\left(\frac{a_{0} n_{0} q_{20}}{p_{0}^{3}}\right)\left[3 \eta_{0}\left(3 \cos ^{2} i_{0}-1\right)+4 K\left(5 \cos ^{2} i_{0}-1\right)\right] \\
& \Phi_{\delta \bar{e}, 33}=1 \\
& \Phi_{\delta \tilde{\mathbf{e}}, 41}=\frac{7 \sigma}{8}\left(\frac{n_{0}}{a_{0} p_{0}^{2}}\right)\left\{q_{10} \sin \left(\dot{\omega}^{(s)}\left(t-t_{0}\right)\right)+q_{20} \cos \left(\dot{\omega}^{(s)}\left(t-t_{0}\right)\right)\right\} \times \\
& \left(5 \cos ^{2} i_{0}-1\right)\left(t-t_{0}\right) \\
& \Phi_{\delta \bar{e}, 43}=\frac{5 \sigma}{2}\left(\frac{n_{0}}{p_{0}^{2}}\right)\left\{q_{10} \sin \left(\dot{\omega}^{(s)}\left(t-t_{0}\right)\right)+q_{20} \cos \left(\dot{\omega}^{(s)}\left(t-t_{0}\right)\right)\right\} \times \\
& \left(\sin i_{0} \cos i_{0}\right)\left(t-t_{0}\right) \\
& \Phi_{\delta \bar{e}, 44}=\cos \left(\dot{\omega}^{(s)}\left(t-t_{0}\right)\right)-\sigma\left(\frac{a_{0} n_{0} q_{10}}{p_{0}^{3}}\right)\left\{q_{10} \sin \left(\dot{\omega}^{(s)}\left(t-t_{0}\right)\right)+\right. \\
& \left.q_{20} \cos \left(\dot{\omega}^{(s)}\left(t-t_{0}\right)\right)\right\}\left(5 \cos ^{2} i_{0}-1\right)\left(t-t_{0}\right) \\
& \Phi_{\delta \bar{e}, 45}=-\sin \left(\dot{\omega}^{(s)}\left(t-t_{0}\right)\right)-\sigma\left(\frac{a_{0} n_{0} q_{20}}{p_{0}^{3}}\right)\left\{q_{10} \sin \left(\dot{\omega}^{(s)}\left(t-t_{0}\right)\right)+\right. \\
& \left.q_{20} \cos \left(\dot{\omega}^{(s)}\left(t-t_{0}\right)\right)\right\}\left(5 \cos ^{2} i_{0}-1\right)\left(t-t_{0}\right) \\
& \Phi_{\delta \bar{e}, 51}=-\frac{7 \sigma}{8}\left(\frac{n_{0}}{a_{0} p_{0}^{2}}\right)\left\{q_{10} \cos \left(\dot{\omega}^{(s)}\left(t-t_{0}\right)\right)-q_{20} \sin \left(\dot{\omega}^{(s)}\left(t-t_{0}\right)\right)\right\} \times \\
& \left(5 \cos ^{2} i_{0}-1\right)\left(t-t_{0}\right) \\
& \Phi_{\delta \bar{e}, 53}=-\frac{5 \sigma}{2}\left(\frac{n_{0}}{p_{0}^{2}}\right)\left\{q_{10} \cos \left(\dot{\omega}^{(s)}\left(t-t_{0}\right)\right)-q_{20} \sin \left(\dot{\omega}^{(s)}\left(t-t_{0}\right)\right)\right\} \times \\
& \left(\sin i_{0} \cos i_{0}\right)\left(t-t_{0}\right) \\
& \Phi_{\delta \bar{e}, 54}=\sin \left(\dot{\omega}^{(s)}\left(t-t_{0}\right)\right)+\sigma\left(\frac{a_{0} n_{0} q_{10}}{p_{0}^{3}}\right)\left\{q_{10} \cos \left(\dot{\omega}^{(s)}\left(t-t_{0}\right)\right)-\right. \\
& \left.q_{20} \sin \left(\dot{\omega}^{(s)}\left(t-t_{0}\right)\right)\right\}\left(5 \cos ^{2} i_{0}-1\right)\left(t-t_{0}\right)
\end{aligned}
$$




$$
\begin{aligned}
\Phi_{\delta \bar{e}, 55}= & \cos \left(\dot{\omega}^{(s)}\left(t-t_{0}\right)\right)+\sigma\left(\frac{a_{0} n_{0} q_{20}}{p_{0}^{3}}\right)\left\{q_{10} \cos \left(\dot{\omega}^{(s)}\left(t-t_{0}\right)\right)-\right. \\
& \left.q_{20} \sin \left(\dot{\omega}^{(s)}\left(t-t_{0}\right)\right)\right\}\left(5 \cos ^{2} i_{0}-1\right)\left(t-t_{0}\right) \\
\Phi_{\delta \bar{e}, 61}= & \frac{7 \sigma}{4}\left(\frac{n_{0} \cos i_{0}}{a_{0} p_{0}^{2}}\right)\left(t-t_{0}\right) \\
\Phi_{\delta \bar{e}, 63}= & \frac{\sigma}{2}\left(\frac{n_{0} \sin i_{0}}{p_{0}^{2}}\right)\left(t-t_{0}\right) \\
\Phi_{\delta \bar{e}, 64}= & -2 \sigma\left(\frac{n_{0} a_{0} q_{10} \cos i_{0}}{p_{0}^{3}}\right)\left(t-t_{0}\right) \\
\Phi_{\delta \bar{e}, 65}= & -2 \sigma\left(\frac{n_{0} a_{0} q_{20} \cos i_{0}}{p_{0}^{3}}\right)\left(t-t_{0}\right) \\
\Phi_{\delta \bar{e}, 66}= & 1
\end{aligned}
$$

where

$$
\begin{aligned}
G_{\theta} & =\frac{\partial G}{\partial \theta}=\frac{n r}{V_{t}} \\
G_{\theta_{0}} & =\frac{\partial G}{\partial \theta_{0}}=-\frac{n_{0} r_{0}}{V_{t 0}} \\
G_{q_{1}} & =\frac{\partial G}{\partial q_{1}}=\frac{q_{2}}{\eta(1+\eta)}+\frac{q_{1} V_{r}}{\eta V_{t}}-\frac{\eta r(a+r)}{p^{2}}\left(q_{2}+\sin \theta\right) \\
G_{q_{10}} & =\frac{\partial G}{\partial q_{10}}=-\frac{q_{20}}{\eta_{0}\left(1+\eta_{0}\right)}-\frac{q_{10} V_{r 0}}{\eta_{0} V_{t 0}}+\frac{\eta_{0} r_{0}\left(a_{0}+r_{0}\right)}{p_{0}^{2}}\left(q_{20}+\sin \theta_{0}\right) \\
G_{q_{2}}= & \frac{\partial G}{\partial q_{2}}=-\frac{q_{1}}{\eta(1+\eta)}+\frac{q_{2} V_{r}}{\eta V_{t}}+\frac{\eta r(a+r)}{p^{2}}\left(q_{1}+\cos \theta\right) \\
G_{q_{20}}= & \frac{\partial G}{\partial q_{20}}=\frac{q_{10}}{\eta_{0}\left(1+\eta_{0}\right)}-\frac{q_{20} V_{r 0}}{\eta_{0} V_{t 0}}-\frac{\eta_{0} r_{0}\left(a_{0}+r_{0}\right)}{p_{0}^{2}}\left(q_{10}+\cos \theta_{0}\right) \\
\dot{\omega}^{(s)}= & \frac{3}{4} J_{2}\left(\frac{R_{e}}{p_{0}}\right)^{2} n_{0}\left(5 \cos ^{2} i_{0}-1\right) \\
K= & 1+G_{q_{1}}\left\{q_{10} \sin \left(\dot{\omega}^{(s)}\left(t-t_{0}\right)\right)+q_{20} \cos \left(\dot{\omega}^{(s)}\left(t-t_{0}\right)\right)\right\}- \\
& G_{q_{2}}\left\{q_{10} \cos \left(\dot{\omega}^{(s)}\left(t-t_{0}\right)\right)-q_{20} \sin \left(\dot{\omega}^{(s)}\left(t-t_{0}\right)\right)\right\} \\
n= & \sqrt{\frac{\mu}{a^{3}}} \\
n_{0}= & \sqrt{\frac{\mu}{a_{0}^{3}}}
\end{aligned}
$$




$$
\begin{aligned}
p & =a\left(1-q_{1}^{2}-q_{2}^{2}\right) \\
p_{0} & =a\left(1-q_{10}^{2}-q_{20}^{2}\right) \\
\eta & =\sqrt{1-q_{1}^{2}-q_{2}^{2}} \\
\eta_{0} & =\sqrt{1-q_{10}^{2}-q_{20}^{2}} \\
V_{r} & =\sqrt{\frac{\mu}{p}}\left(q_{1} \sin \theta-q_{2} \cos \theta\right) \\
V_{r 0} & =\sqrt{\frac{\mu}{p_{0}}}\left(q_{10} \sin \theta_{0}-q_{20} \cos \theta_{0}\right) \\
V_{t} & =\sqrt{\frac{\mu}{p}}\left(1+q_{1} \cos \theta+q_{2} \sin \theta\right) \\
V_{t 0} & =\sqrt{\frac{\mu}{p_{0}}}\left(1+q_{10} \cos \theta_{0}+q_{20} \sin \theta_{0}\right)
\end{aligned}
$$




\section{Appendix E}

\section{Orbit Elements Transformation Column Vectors $\boldsymbol{e}^{(l p)}, \boldsymbol{e}^{(s p 1)}$, and $\boldsymbol{e}^{(s p 2)}$}

The long period oscillation $\boldsymbol{e}^{(l p)}$ and the short period oscillations $\boldsymbol{e}^{(s p 1)}$ and $\boldsymbol{e}^{(s p 2)}$ in the osculating orbit elements caused by the $J_{2}$ graviational perturbation were mathematically formulated first time by professor Kyle T. Alfriend and his research associate Dr. Dong-Woo Gim from Texas A\&M university. Professor Alfriend and Dr. Gim presented vectors $e^{(l p)}, e^{(s p 1)}$, and $e^{(s p 2)}$ on their renowned 2003 paper ${ }^{[16]}$ which was published in the Journal of Guidance, Control and Dynamics. I utilized Maxima computer algebra system for symbolic manipulation of mathematical expressions and rederived these column vectors to verify the accuracy of Gim-Alfriend $\boldsymbol{e}^{(l p)}, \boldsymbol{e}^{(s p 1)}$, and $\boldsymbol{e}^{(s p 2)}$ components expressions. The elements of my developed long and short period variations $\Phi_{\delta \bar{e}}\left(t, t_{0}\right)$ are as follows. There is no major difference in component expressions between Gim-Alfriend produced mean state transition matrix and my derived matrix. 


\section{E.1 Long Period Oscillations Column Vector $\boldsymbol{e}^{(l p)}$}

The elements of column vector $\boldsymbol{e}^{(l p)}$ are

$$
\begin{aligned}
a^{(l p)}= & 0 \\
\theta^{(l p)}= & \lambda^{(l p)}-\left(\frac{\sin ^{2} i}{16 a^{2} \eta^{4}}\right)\left(1-10 \Theta \cos ^{2} i\right)\left\{q_{1} q_{2}\left[3+\frac{2 \eta^{2}}{(1+\eta)}\right]+\right. \\
& \left.2\left(q_{1} \sin \theta+q_{2} \cos \theta\right)+\frac{\epsilon_{1} \sin 2 \theta}{2}\right\} \\
i^{(l p)}= & \left(\frac{\sin 2 i}{32 a^{2} \eta^{4}}\right)\left(1-10 \Theta \cos ^{2} i\right)\left(q_{1}^{2}-q_{2}^{2}\right) \\
q_{1}^{(l p)}= & -\left(\frac{q_{1} \sin ^{2} i}{16 a^{2} \eta^{2}}\right)\left(1-10 \Theta \cos ^{2} i\right)-\left(\frac{q_{1} q_{2}^{2}}{16 a^{2} \eta^{4}}\right)\left\{3-55 \cos ^{2} i-\right. \\
& \left.280 \Theta \cos ^{4} i-400 \Theta^{2} \cos ^{6} i\right\} \\
q_{2}^{(l p)}= & \left(\frac{q_{2} \sin ^{2} i}{16 a^{2} \eta^{2}}\right)\left(1-10 \Theta \cos ^{2} i\right)+\left(\frac{q_{1}^{2} q_{2}}{16 a^{2} \eta^{4}}\right)\left\{3-55 \cos ^{2} i-\right. \\
& \left.280 \Theta \cos ^{4} i-400 \Theta^{2} \cos ^{6} i\right\} \\
\Omega^{(l p)}= & \left(\frac{q_{1} q_{2} \cos ^{6} i}{8 a^{2} \eta^{4}}\right)\left\{11+80 \Theta \cos ^{2} i+200 \Theta^{2} \cos ^{4} i\right\}
\end{aligned}
$$

where

$$
\begin{aligned}
\lambda^{(l p)}= & {\left[\frac{q_{1} q_{2} \sin ^{2} i}{8 a^{2} \eta^{2}(1+\eta)}\right]\left(1-10 \Theta \cos ^{2} i\right)+} \\
& \left(\frac{q_{1} q_{2}}{16 a^{2} \eta^{4}}\right)\left\{3-55 \cos ^{2} i-280 \Theta \cos ^{4} i-400 \Theta^{2} \cos ^{6} i\right\} \\
\Theta & =\frac{1}{\left(1-5 \cos ^{2} i\right)} \\
\epsilon_{1} & =\sqrt{q_{1}^{2}+q_{2}^{2}} \\
\eta & =\sqrt{1-q_{1}^{2}-q_{2}^{2}}
\end{aligned}
$$




\section{E.2 Short Period 1 Oscillations Column Vector $\boldsymbol{e}^{(s p 1)}$}

The elements of column vector $\boldsymbol{e}^{(s p 1)}$ are

$$
\begin{aligned}
a^{(s p 1)}= & {\left[\frac{1-3 \cos ^{2} i}{2 a \eta^{6}}\right]\left[\left(1+\epsilon_{2}\right)^{3}-\eta^{3}\right] } \\
\theta^{(s p 1)}= & \lambda^{(s p 1)}-\left[\frac{\epsilon_{3}\left(1-3 \cos ^{2} i\right)}{4 a^{2} \eta^{4}(1+\eta)}\right]\left[\left(1+\epsilon_{2}\right)^{2}+\eta(1+\eta)\right] \\
i^{(s p 1)}= & 0 \\
q_{1}^{(s p 1)}= & {\left[\frac{1-3 \cos ^{2} i}{4 a^{2} \eta^{4}(1+\eta)}\right]\left\{\left[\left(1+\epsilon_{2}\right)^{2}+\eta^{2}\right]\left[q_{1}+(1+\eta) \cos \theta\right]+\right.} \\
& \left.\left(1+\epsilon_{2}\right)\left[(1+\eta) \cos \theta+q_{1}\left(\eta-\epsilon_{2}\right)\right]\right\}-\left[\frac{3 q_{2}\left(1-5 \cos ^{2} i\right)}{4 a^{2} \eta^{4}}\right] \times \\
& \left(\theta-\lambda+\epsilon_{3}\right) \\
q_{2}^{(s p 1)}= & {\left[\frac{1-3 \cos ^{2} i}{4 a^{2} \eta^{4}(1+\eta)}\right]\left\{\left[\left(1+\epsilon_{2}\right)^{2}+\eta^{2}\right]\left[q_{2}+(1+\eta) \sin \theta\right]+\right.} \\
& \left.\left(1+\epsilon_{2}\right)\left[(1+\eta) \sin \theta+q_{2}\left(\eta-\epsilon_{2}\right)\right]\right\}+\left[\frac{3 q_{1}\left(1-5 \cos ^{2} i\right)}{4 a^{2} \eta^{4}}\right] \times \\
& \left(\theta-\lambda+\epsilon_{3}\right) \\
\Omega^{(s p 1)}= & \left(\frac{3 \cos i}{2 a^{2} \eta^{4}}\right)\left(\theta-\lambda+\epsilon_{3}\right)
\end{aligned}
$$

where

$$
\begin{aligned}
\lambda^{(s p 1)} & =\left[\frac{\epsilon_{3}\left(1-3 \cos ^{2} i\right)}{4 a^{2} \eta^{4}(1+\eta)}\right]\left[\left(1+\epsilon_{2}\right)^{2}+\left(1+\epsilon_{2}\right)+\eta^{2}\right]+ \\
\lambda & {\left[\frac{3\left(1-5 \cos ^{2} i\right)}{4 a^{2} \eta^{4}}\right]\left(\theta-\lambda+\epsilon_{3}\right) } \\
r & =\frac{a \eta^{2}}{1+\epsilon_{2}}\left[\frac{r\left(1+\beta q_{1}^{2}\right) \sin \theta-\beta r q_{1} q_{2} \cos \theta+a q_{2}}{r\left(1+\beta q_{2}^{2}\right) \cos \theta-\beta r q_{1} q_{2} \sin \theta+a q_{1}}\right]-\frac{\eta \epsilon_{3}}{1+\epsilon_{2}} \\
\beta & =\frac{1}{\eta^{2}+\eta} \\
\epsilon_{2} & =q_{1} \cos \theta+q_{2} \sin \theta
\end{aligned}
$$




$$
\begin{gathered}
\epsilon_{3}=q_{1} \sin \theta-q_{2} \cos \theta \\
\eta=\sqrt{1-q_{1}^{2}-q_{2}^{2}}
\end{gathered}
$$

\section{E.3 Short Period 2 Oscillations Column Vector $\boldsymbol{e}^{(s p 2)}$}

The elements of column vector $e^{(s p 2)}$ are

$$
\begin{aligned}
a^{(s p 2)}= & -\left(\frac{3 \sin ^{2} i}{2 a \eta^{6}}\right)\left(1+\epsilon_{2}\right)^{3} \cos 2 \theta \\
\theta^{(s p 2)}= & \lambda^{(s p 2)}-\left[\frac{\sin ^{2} i}{32 a^{2} \eta^{4}(1+\eta)}\right]\left\{36 q_{1} q_{2}-4\left(3 \eta^{2}+5 \eta-1\right) \times\right. \\
& \left(q_{1} \sin \theta+q_{2} \cos \theta\right)+12 \epsilon_{2} q_{1} q_{2}-32(1+\eta) \sin 2 \theta- \\
& \left(\eta^{2}+12 \eta+39\right)\left(q_{1} \sin 3 \theta-q_{2} \cos 3 \theta\right)+36 q_{1} q_{2} \cos 4 \theta- \\
& \left.18\left(q_{1}^{2}-q_{2}^{2}\right) \sin 4 \theta-3\left(q_{1}^{2}-3 q_{2}^{2}\right) q_{1} \sin 5 \theta+3\left(3 q_{1}^{2}-q_{2}^{2}\right) q_{2} \cos 5 \theta\right\} \\
i^{(s p 2)=}- & \left(\frac{\sin 2 i}{8 a^{2} \eta^{4}}\right)\left\{3\left(q_{1} \cos \theta-q_{2} \sin \theta\right)+3 \cos 2 \theta+\right. \\
& \left.\left(q_{1} \cos 3 \theta+q_{2} \sin 3 \theta\right)\right\} \\
q_{1}^{(s p 2)}= & {\left[\frac{q_{2}\left(3-5 \cos { }^{2} i\right)}{8 a^{2} \eta^{4}}\right]\left\{3\left(q_{1} \sin \theta+q_{2} \cos \theta\right)+3 \sin 2 \theta+\right.} \\
& \left.\left(q_{1} \sin 3 \theta-q_{2} \cos 3 \theta\right)\right\}+\left(\frac{\sin i}{8 a^{2} \eta^{4}}\right)\left\{3\left(\eta^{2}-q_{1}^{2}\right) \cos \theta+3 q_{1} q_{2} \sin \theta-\right. \\
& \left.\left(\eta^{2}+3 q_{1}^{2}\right) \cos 3 \theta-3 q_{1} q_{2} \sin 3 \theta\right\}-\left(\frac{3 \sin ^{2} i \cos 2 \theta}{16 a^{2} \eta^{4}}\right)\left\{10 q_{1}+\right. \\
& \left(8+3 q_{1}^{2}+q_{2}^{2}\right) \cos \theta+2 q_{1} q_{2} \sin \theta+6\left(q_{1} \cos 2 \theta+q_{2} \sin 2 \theta\right)+ \\
& \left.\left(q_{1}^{2}-q_{2}^{2}\right) \cos 3 \theta+2 q_{1} q_{2} \sin 3 \theta\right\}
\end{aligned}
$$




$$
\begin{aligned}
q_{2}^{(s p 2)}= & -\left[\frac{q_{1}\left(3-5 \cos ^{2} i\right)}{8 a^{2} \eta^{4}}\right]\left\{3\left(q_{1} \sin \theta+q_{2} \cos \theta\right)+3 \sin 2 \theta+\right. \\
& \left.\left(q_{1} \sin 3 \theta-q_{2} \cos 3 \theta\right)\right\}-\left(\frac{\sin ^{2} i}{8 a^{2} \eta^{4}}\right)\left\{3\left(\eta^{2}-q_{2}^{2}\right) \sin \theta+3 q_{1} q_{2} \cos \theta+\right. \\
& \left.\left(\eta^{2}+3 q_{2}^{2}\right) \sin 3 \theta+3 q_{1} q_{2} \cos 3 \theta\right\}-\left(\frac{3 \sin ^{2} i \cos 2 \theta}{16 a^{2} \eta^{4}}\right)\left\{10 q_{2}+\right. \\
& \left(8+q_{1}^{2}+3 q_{2}^{2}\right) \sin \theta+2 q_{1} q_{2} \cos \theta+6\left(q_{1} \sin 2 \theta-q_{2} \cos 2 \theta\right)+ \\
& \left.\left(q_{1}^{2}-q_{2}^{2}\right) \sin 3 \theta-2 q_{1} q_{2} \cos 3 \theta\right\} \\
\Omega^{(s p 2)}= & -\left(\frac{\cos i}{4 a^{2} \eta^{4}}\right)\left\{3\left(q_{1} \sin \theta+q_{2} \cos \theta\right)+3 \sin 2 \theta+\left(q_{1} \sin 3 \theta-q_{2} \cos 3 \theta\right)\right\}
\end{aligned}
$$

where

$$
\begin{aligned}
& \lambda^{(s p 2)}=-\left[\frac{3 \epsilon_{3} \sin ^{2} i \cos 2 \theta}{4 a^{2} \eta^{4}(1+\eta)}\right]\left(1+\epsilon_{2}\right)\left(2+\epsilon_{2}\right)-\left[\frac{\sin ^{2} i}{8 a^{2} \eta^{2}(1+\eta)}\right] \times \\
& {\left[3\left(q_{1} \sin \theta+q_{2} \cos \theta\right)+\left(q_{1} \sin 3 \theta-q_{2} \cos 3 \theta\right)\right]-\left[\frac{3-5 \cos ^{2} i}{8 a^{2} \eta^{4}}\right] \times } \\
& {\left[3\left(q_{1} \sin \theta+q_{2} \cos \theta\right)+3 \sin 2 \theta+\left(q_{1} \sin 3 \theta-q_{2} \cos 3 \theta\right)\right] } \\
& \epsilon_{2}= q_{1} \cos \theta+q_{2} \sin \theta \\
& \epsilon_{3}= q_{1} \sin \theta-q_{2} \cos \theta \\
& \eta= \sqrt{1-q_{1}^{2}-q_{2}^{2}}
\end{aligned}
$$




\section{Appendix F}

\section{Relative Orbit Elements Transformation Matrices $D^{(l p)}$, $D^{(s p 1)}$, and $D^{(s p 2)}$}

Professor Kyle T. Alfriend and his research associate Dr. Dong-Woo Gim from Texas A\&M university presented the following relative orbit elements transformation matrices $D^{(l p)}, D^{(s p 1)}$, and $D^{(s p 2)}$ on their renowned 2003 paper ${ }^{[16]}$ which was published in the Journal of Guidance, Control and Dynamics.

\section{F.1 Long Period Oscillations Matrix $D^{(l p)}$}

The non-zero elements of matrix $D^{(l p)}$ are

$$
\begin{aligned}
D_{11}^{(l p)}= & -\left(\frac{1}{a}\right) a^{(l p)} \\
D_{21}^{(l p)}= & -\left(\frac{2}{a}\right) \theta^{(l p)} \\
D_{22}^{(l p)}= & -\left(\frac{\sin ^{2} i}{16 a^{2} \eta^{4}}\right)\left(1-10 \Theta \cos ^{2} i\right)\left[2\left(q_{1} \cos \theta-q_{2} \sin \theta\right)+\epsilon_{1} \cos 2 \theta\right] \\
D_{23}^{(l p)}= & \left(\frac{\sin 2 i}{16 a^{2} \eta^{4}}\right)\left\{5 q_{1} q_{2}\left(11+112 \Theta \cos ^{2} i+520 \Theta^{2} \cos ^{4} i+800 \Theta^{3} \cos ^{6} i\right)-\right. \\
& {\left[2 q_{1} q_{2}+\left(2+\epsilon_{2}\right)\left(q_{1} \sin \theta+q_{2} \cos \theta\right)\right]\left[\left(1-10 \Theta \cos ^{2} i\right)+\right.} \\
& \left.\left.10 \Theta \sin ^{2} i\left(1+5 \Theta \cos ^{2} i\right)\right]\right\}
\end{aligned}
$$




$$
\begin{aligned}
D_{24}^{(l p)}= & \left(\frac{1}{16 a^{2} \eta^{6}}\right)\left\{( \eta ^ { 2 } + 4 q _ { 1 } ^ { 2 } ) \left[q_{2}\left(3-55 \cos ^{2} i-280 \Theta \cos ^{4} i-400 \Theta^{2} \cos ^{6} i\right)-\right.\right. \\
& \left.\sin ^{2} i\left(1-10 \Theta \cos ^{2} i\right)\left(3 q_{2}+2 \sin \theta\right)\right]-2 \sin ^{2} i\left(1-10 \Theta \cos ^{2} i\right) \times \\
& {\left.\left[4 q_{2}+\sin \theta\left(1+\epsilon_{1}\right)\right] q_{1} \cos \theta\right\} } \\
D_{25}^{(l p)}= & \left(\frac{1}{16 a^{2} \eta^{6}}\right)\left\{( \eta ^ { 2 } + 4 q _ { 2 } ^ { 2 } ) \left[q_{1}\left(3-55 \cos ^{2} i-280 \Theta \cos ^{4} i-400 \Theta^{2} \cos ^{6} i\right)-\right.\right. \\
& \left.\sin ^{2} i\left(1-10 \Theta \cos ^{2} i\right)\left(3 q_{1}+2 \cos \theta\right)\right]-2 \sin ^{2} i\left(1-10 \Theta \cos ^{2} i\right) \times \\
& {\left.\left[4 q_{1}+\cos \theta\left(1+\epsilon_{1}\right)\right] q_{2} \sin \theta\right\} } \\
D_{31}^{(l p)}= & -\left(\frac{2}{a}\right) i^{(l p)} \\
D_{33}^{(l p)}= & \left(\frac{q_{1}^{2}-q_{2}^{2}}{16 a^{2} \eta^{4}}\right)\left[\cos ^{2} 2 i\left(1-10 \Theta \cos ^{2} i\right)+5 \Theta \sin ^{2} 2 i\left(1+5 \Theta \cos ^{2} i\right)\right] \\
D_{34}^{(l p)}= & \left(\frac{q_{1} \sin 2 i}{16 a^{2} \eta^{6}}\right)\left(1-10 \Theta \cos ^{2} i\right)\left[\eta^{2}+2\left(q_{1}^{2}-q_{2}^{2}\right)\right] \\
D_{51}^{(l p)}= & -\left(\frac{2}{a}\right) q_{2}^{(l p)} \\
D_{53}^{(l p)}= & \left(\frac{q_{2} \sin 2 i}{16 a^{2} \eta^{4}}\right)\left[\eta^{2}\left(1-10 \Theta \cos ^{2} i\right)+10 \Theta \eta^{2} \sin ^{2} i\left(1+5 \Theta \cos ^{2} i\right)+\right. \\
& \left(\frac{q_{2} \sin 2 i}{16 a^{2} \eta^{6}}\right)\left(1-10 \Theta \cos ^{2} i\right)\left[\eta^{2}-2\left(q_{1}^{2}-q_{2}^{2}\right)\right] \\
D_{41}^{(l p)}= & -\left(\frac{2}{a}\right) q_{1}^{(l p)} \\
D_{45}^{(l p)}= & -\left(\frac{1}{16 a^{2} \eta^{6}}\right)\left\{\eta^{2} \sin ^{2} i\left(1-112 \Theta \cos ^{2} i+520 \Theta^{2} \cos ^{4} i+800 \Theta^{3} \cos ^{6} i\right)\right] \\
D_{43}^{(l p)}= & \left.\left.-\left(\frac{q_{1} \sin 2 i}{16 a^{2} \eta^{4}}\right)\left\{\eta^{2}\left[\left(1-10 \Theta \cos ^{2} i\right)+10 \Theta \sin ^{2} i\left(1+5 \Theta \cos ^{2} i\right)\right]+280 \Theta \cos ^{4} i-400 \Theta^{2} \cos ^{6} i\right)\left(\eta^{2}+2 q_{1}^{2}\right)+4 q_{1}^{2}\right)\right\} \\
\left.5 q_{2}^{2}\left(11+112 \Theta \cos ^{2} i+520 \Theta^{2} \cos ^{4} i+800 \Theta^{3} \cos ^{6} i\right)\right\} & (\mathrm{F} .1 \mathrm{~g})\left[\eta^{2} \sin ^{2} i\left(1-10 \Theta \cos ^{2} i\right)+\right. \\
\left.D^{2}\right) & (\mathrm{F} .1 \mathrm{k})
\end{aligned}
$$




$$
\begin{aligned}
D_{54}^{(l p)}= & \left(\frac{q_{1} q_{2}}{8 a^{2} \eta^{6}}\right)\left[\eta^{2} \sin ^{2} i\left(1-10 \Theta \cos ^{2} i\right)+\right. \\
& \left.\left(3-55 \cos ^{2} i-280 \Theta \cos ^{4} i-400 \Theta^{2} \cos ^{6} i\right)\left(\eta^{2}+2 q_{1}^{2}\right)\right] \\
D_{55}^{(l p)}= & \left(\frac{1}{16 a^{2} \eta^{6}}\right)\left\{\eta^{2} \sin ^{2} i\left(1-10 \Theta \cos ^{2} i\right)\left(\eta^{2}+2 q_{2}^{2}\right)+\right. \\
& \left.q_{1}^{2}\left(3-55 \cos ^{2} i-280 \Theta \cos ^{4} i-400 \Theta^{2} \cos ^{6} i\right)\left(\eta^{2}+4 q_{2}^{2}\right)\right\} \\
D_{61}^{(l p)}= & -\left(\frac{2}{a}\right) \Omega^{(l p)} \\
D_{63}^{(l p)}= & -\left(\frac{q_{1} q_{2} \sin i}{8 a^{2} \eta^{4}}\right)\left[\left(11+80 \Theta \cos ^{2} i+200 \Theta^{2} \cos ^{4} i\right)+\right. \\
& \left.160 \Theta \cos ^{2} i\left(1+5 \Theta \cos ^{2} i\right)^{2}\right] \\
D_{64}^{(l p)}= & \left(\frac{q_{2} \cos i}{8 a^{2} \eta^{6}}\right)\left(11+80 \Theta \cos ^{2} i+200 \Theta^{2} \cos ^{4} i\right)\left(\eta^{2}+4 q_{1}^{2}\right) \\
D_{65}^{(l p)}= & \left(\frac{q_{1} \cos i}{8 a^{2} \eta^{6}}\right)\left(11+80 \Theta \cos ^{2} i+200 \Theta^{2} \cos ^{4} i\right)\left(\eta^{2}+4 q_{2}^{2}\right)
\end{aligned}
$$

where

$$
\begin{aligned}
\Theta & =\frac{1}{\left(1-5 \cos ^{2} i\right)} \\
\epsilon_{1} & =\sqrt{q_{1}^{2}+q_{2}^{2}} \\
\epsilon_{2} & =q_{1} \cos \theta+q_{2} \sin \theta \\
\eta & =\sqrt{1-q_{1}^{2}-q_{2}^{2}}
\end{aligned}
$$

Note that the expressions for the orbit elements variations $e^{(l p)}=\left(a^{(l p)}, \theta^{(l p)}, i^{(l p)}\right.$, $\left.q_{1}^{(l p)}, q_{2}^{(l p)}, \Omega^{(l p)}\right)^{T}$ are given in appendix E. 


\section{F.2 Short Period 1 Oscillations Matrix $D^{(s p 1)}$}

The non-zero elements of matrix $D^{(s p 1)}$ are

$$
\begin{aligned}
& D_{11}^{(s p 1)}=-\left(\frac{1}{a}\right) a^{(s p 1)} \\
& D_{12}^{(s p 1)}=-\left(\frac{3 \epsilon_{3}}{2 a \eta^{6}}\right)\left(1-3 \cos ^{2} i\right)\left(1+\epsilon_{2}\right)^{2} \\
& D_{13}^{(s p 1)}=\left(\frac{3 \sin 2 i}{2 a \eta^{6}}\right)\left[\left(1+\epsilon_{2}\right)^{3}-\eta^{3}\right] \\
& D_{14}^{(s p 1)}=\left[\frac{3\left(1-3 \cos ^{2} i\right)}{2 a \eta^{8}}\right]\left[2 q_{1}\left(1+\epsilon_{2}\right)^{3}+\eta^{2}\left(1+\epsilon_{2}\right)^{2} \cos \theta-\eta^{3} q_{1}\right] \\
& D_{15}^{(s p 1)}=\left[\frac{3\left(1-3 \cos ^{2} i\right)}{2 a \eta^{8}}\right]\left[2 q_{2}\left(1+\epsilon_{2}\right)^{3}+\eta^{2}\left(1+\epsilon_{2}\right)^{2} \sin \theta-\eta^{3} q_{2}\right] \\
& D_{21}^{(s p 1)}=-\left(\frac{2}{a}\right) \theta^{(s p 1)} \\
& D_{22}^{(s p 1)}=\left[\frac{1-3 \cos ^{2} i}{4 a^{2} \eta^{4}(1+\eta)}\right]\left[\epsilon_{2}\left(1+\epsilon_{2}-\eta\right)-\epsilon_{3}^{2}\right]+\left[\frac{3\left(1-5 \cos ^{2} i\right)}{4 a^{2} \eta^{4}\left(1+\epsilon_{2}\right)^{2}}\right] \times \\
& {\left[\left(1+\epsilon_{2}\right)^{3}-\eta^{3}\right]} \\
& D_{23}^{(s p 1)}=\left[\frac{3 \epsilon_{3} \sin 2 i}{4 a^{2} \eta^{4}(1+\eta)}\right]\left[\left(1+\epsilon_{2}\right)+(5+4 \eta)\right]+\left(\frac{15 \sin 2 i}{4 a^{2} \eta^{4}}\right)(\theta-\lambda) \\
& D_{24}^{(s p 1)}=\left[\frac{1-3 \cos ^{2} i}{4 a^{2} \eta^{6}(1+\eta)^{2}}\right]\left\{\eta^{2}\left[\epsilon_{1} \sin \theta+(1+\eta)\left(\epsilon_{2} \sin \theta+\epsilon_{3} \cos \theta\right)\right]+\right. \\
& \left.q_{1} \epsilon_{3}\left[4\left(\epsilon_{1}+\epsilon_{2}\right)+\eta\left(2+5 \epsilon_{2}\right)\right]\right\}+\left[\frac{3\left(1-5 \cos ^{2} i\right)}{4 a^{2} \eta^{6}}\right] \times \\
& {\left[4 q_{1}\left(\theta-\lambda+\epsilon_{3}\right)+\eta^{2} \sin \theta\right]-\left[\frac{3\left(1-5 \cos ^{2} i\right)}{4 a^{2} \eta^{4}}\right] \lambda_{q_{1}}} \\
& D_{25}^{(s p 1)}=-\left[\frac{1-3 \cos ^{2} i}{4 a^{2} \eta^{6}(1+\eta)^{2}}\right]\left\{\eta^{2}\left[\epsilon_{1} \cos \theta+(1+\eta)\left(\epsilon_{2} \cos \theta-\epsilon_{3} \sin \theta\right)\right]-\right. \\
& \left.q_{2} \epsilon_{3}\left[4\left(\epsilon_{1}+\epsilon_{2}\right)+\eta\left(2+5 \epsilon_{2}\right)\right]\right\}+\left[\frac{3\left(1-5 \cos ^{2} i\right)}{4 a^{2} \eta^{6}}\right] \times \\
& {\left[4 q_{2}\left(\theta-\lambda+\epsilon_{3}\right)-\eta^{2} \cos \theta\right]-\left[\frac{3\left(1-5 \cos ^{2} i\right)}{4 a^{2} \eta^{4}}\right] \lambda_{q_{2}}} \\
& D_{31}^{(s p 1)}=-\left(\frac{2}{a}\right) i^{(s p 1)} \\
& D_{41}^{(s p 1)}=-\left(\frac{2}{a}\right) q_{1}^{(s p 1)}
\end{aligned}
$$




$$
\begin{aligned}
& D_{42}^{(s p 1)}=-\left[\frac{1-3 \cos ^{2} i}{4 a^{2} \eta^{4}}\right]\left[\left(1+\epsilon_{2}\right)\left(2 \sin \theta+\epsilon_{2} \sin \theta+2 \epsilon_{3} \cos \theta\right)+\epsilon_{3}\left(q_{1}+\cos \theta\right)+\right. \\
& \left.\eta^{2} \sin \theta\right]-\left[\frac{3 q_{2}\left(1-5 \cos ^{2} i\right)}{4 a^{2} \eta^{4}\left(1+\epsilon_{2}\right)^{2}}\right]\left[\left(1+\epsilon_{2}\right)^{3}-\eta^{3}\right] \\
& D_{43}^{(s p 1)}=\left[\frac{3 q_{1} \sin 2 i}{4 a^{2} \eta^{2}(1+\eta)}\right]+\left(\frac{3 \sin 2 i}{4 a^{2} \eta^{4}}\right)\left\{\left(1+\epsilon_{2}\right)\left[q_{1}+\left(2+\epsilon_{2}\right) \cos \theta\right]-5 q_{2} \epsilon_{3}+\right. \\
& \left.\eta^{2} \cos \theta\right\}-\left(\frac{15 q_{2} \sin 2 i}{4 a^{2} \eta^{4}}\right)(\theta-\lambda) \\
& D_{44}^{(s p 1)}=\left[\frac{1-3 \cos ^{2} i}{4 a^{2} \eta^{2}(1+\eta)}\right]+\left[\frac{1-3 \cos ^{2} i}{8 a^{2} \eta^{6}}\right]\left\{\eta ^ { 2 } \left[5+2\left(5 q_{1} \cos \theta+2 q_{2} \sin \theta\right)+\right.\right. \\
& \left.\left.\left(3+2 \epsilon_{2}\right) \cos 2 \theta\right]+2 q_{1}\left[4\left(1+\epsilon_{2}\right)\left(2+\epsilon_{2}\right) \cos \theta+\left(3 \eta+4 \epsilon_{2}\right) q_{1}\right]\right\}+ \\
& {\left[\frac{\left(1-3 \cos ^{2} i\right) q_{1}^{2}(4+5 \eta)}{4 a^{2} \eta^{6}(1+\eta)^{2}}\right]-\left[\frac{3 q_{2}\left(1-5 \cos ^{2} i\right)}{4 a^{2} \eta^{6}}\right]\left[4 q_{1} \epsilon_{3}+\eta^{2} \sin \theta\right]-} \\
& {\left[\frac{3 q_{1} q_{2}\left(1-5 \cos ^{2} i\right)}{a^{2} \eta^{6}}\right](\theta-\lambda)+\left[\frac{3 q_{2}\left(1-5 \cos ^{2} i\right)}{4 a^{2} \eta^{4}}\right] \lambda_{q_{1}}} \\
& D_{45}^{(s p 1)}=\left[\frac{1-3 \cos ^{2} i}{8 a^{2} \eta^{6}}\right]\left\{\eta^{2}\left[2\left(q_{1} \sin \theta+2 q_{2} \cos \theta\right)+\left(3+2 \epsilon_{2}\right) \sin 2 \theta\right]+\right. \\
& \left.2 q_{2}\left[4\left(1+\epsilon_{2}\right)\left(2+\epsilon_{2}\right) \cos \theta+\left(3 \eta+4 \epsilon_{2}\right) q_{1}\right]\right\}+\left[\frac{\left(1-3 \cos ^{2} i\right) q_{1} q_{2}(4+5 \eta)}{4 a^{2} \eta^{6}(1+\eta)^{2}}\right]- \\
& {\left[\frac{3\left(1-5 \cos ^{2} i\right)}{4 a^{2} \eta^{6}}\right]\left[\epsilon_{3}\left(\eta^{2}+4 q_{2}^{2}\right)-\eta^{2} q_{2} \cos \theta\right]-\left[\frac{3\left(1-5 \cos ^{2} i\right)}{4 a^{2} \eta^{6}}\right] \times} \\
& {\left[\left(\eta^{2}+4 q_{2}^{2}\right)(\theta-\lambda)\right]+\left[\frac{3 q_{2}\left(1-5 \cos ^{2} i\right)}{4 a^{2} \eta^{4}}\right] \lambda_{q_{2}}} \\
& D_{51}^{(s p 1)}=-\left(\frac{2}{a}\right) q_{2}^{(s p 1)} \\
& D_{52}^{(s p 1)}=\left[\frac{1-3 \cos ^{2} i}{4 a^{2} \eta^{4}}\right]\left[\left(1+\epsilon_{2}\right)\left(2 \cos \theta+\epsilon_{2} \cos \theta-2 \epsilon_{3} \sin \theta\right)-\epsilon_{3}\left(q_{2}+\sin \theta\right)+\right. \\
& \left.\eta^{2} \cos \theta\right]+\left[\frac{3 q_{1}\left(1-5 \cos ^{2} i\right)}{4 a^{2} \eta^{4}\left(1+\epsilon_{2}\right)^{2}}\right]\left[\left(1+\epsilon_{2}\right)^{3}-\eta^{3}\right] \\
& D_{53}^{(s p 1)}=\left[\frac{3 q_{2} \sin 2 i}{4 a^{2} \eta^{2}(1+\eta)}\right]+\left(\frac{3 \sin 2 i}{4 a^{2} \eta^{4}}\right)\left\{\left(1+\epsilon_{2}\right)\left[q_{2}+\left(2+\epsilon_{2}\right) \sin \theta\right]+5 q_{1} \epsilon_{3}+\right. \\
& \left.\eta^{2} \sin \theta\right\}-\left(\frac{15 q_{1} \sin 2 i}{4 a^{2} \eta^{4}}\right)(\theta-\lambda)
\end{aligned}
$$




$$
\begin{aligned}
& D_{54}^{(s p 1)}=\left[\frac{1-3 \cos ^{2} i}{8 a^{2} \eta^{6}}\right]\left\{\eta^{2}\left[2\left(2 q_{1} \sin \theta+q_{2} \cos \theta\right)+\left(3+2 \epsilon_{2}\right) \sin 2 \theta\right]+\right. \\
& \left.2 q_{1}\left[4\left(1+\epsilon_{2}\right)\left(2+\epsilon_{2}\right) \sin \theta+\left(3 \eta+4 \epsilon_{2}\right) q_{2}\right]\right\}+\left[\frac{\left(1-3 \cos ^{2} i\right) q_{1} q_{2}(4+5 \eta)}{4 a^{2} \eta^{6}(1+\eta)^{2}}\right]+ \\
& {\left[\frac{3\left(1-5 \cos ^{2} i\right)}{4 a^{2} \eta^{6}}\right]\left[\epsilon_{3}\left(\eta^{2}+4 q_{1}^{2}\right)+\eta^{2} q_{1} \sin \theta\right]+\left[\frac{3\left(1-5 \cos ^{2} i\right)}{4 a^{2} \eta^{6}}\right] \times} \\
& {\left[\left(\eta^{2}+4 q_{1}^{2}\right)(\theta-\lambda)\right]-\left[\frac{3 q_{1}\left(1-5 \cos ^{2} i\right)}{4 a^{2} \eta^{4}}\right] \lambda_{q_{1}}} \\
& D_{55}^{(s p 1)}=\left[\frac{1-3 \cos ^{2} i}{4 a^{2} \eta^{2}(1+\eta)}\right]+\left[\frac{1-3 \cos ^{2} i}{8 a^{2} \eta^{6}}\right]\left\{\eta ^ { 2 } \left[5+2\left(2 q_{1} \cos \theta+5 q_{2} \sin \theta\right)-\right.\right. \\
& \left.\left.\left(3+2 \epsilon_{2}\right) \cos 2 \theta\right]+2 q_{2}\left[4\left(1+\epsilon_{2}\right)\left(2+\epsilon_{2}\right) \sin \theta+\left(3 \eta+4 \epsilon_{2}\right) q_{2}\right]\right\}+ \\
& {\left[\frac{\left(1-3 \cos ^{2} i\right) q_{2}^{2}(4+5 \eta)}{4 a^{2} \eta^{6}(1+\eta)^{2}}\right]+\left[\frac{3 q_{1}\left(1-5 \cos ^{2} i\right)}{4 a^{2} \eta^{6}}\right]\left[4 q_{2} \epsilon_{3}-\eta^{2} \cos \theta\right]+} \\
& {\left[\frac{3 q_{1} q_{2}\left(1-5 \cos ^{2} i\right)}{a^{2} \eta^{6}}\right](\theta-\lambda)-\left[\frac{3 q_{1}\left(1-5 \cos ^{2} i\right)}{4 a^{2} \eta^{4}}\right] \lambda_{q_{2}}} \\
& D_{61}^{(s p 1)}=-\left(\frac{2}{a}\right) \Omega^{(s p 1)} \\
& D_{62}^{(s p 1)}=\left[\frac{3 \cos i}{2 a^{2} \eta^{4}\left(1+\epsilon_{2}\right)^{2}}\right]\left[\left(1+\epsilon_{2}\right)^{3}-\eta^{3}\right] \\
& D_{63}^{(s p 1)}=-\left(\frac{3 \epsilon_{3} \sin i}{2 a^{2} \eta^{4}}\right)-\left(\frac{3 \sin i}{2 a^{2} \eta^{4}}\right)(\theta-\lambda) \\
& D_{64}^{(s p 1)}=\left(\frac{3 \cos i}{2 a^{2} \eta^{6}}\right)\left(4 q_{1} \epsilon_{3}+\eta^{2} \sin \theta\right)+\left(\frac{6 q_{1} \cos i}{a^{2} \eta^{6}}\right)(\theta-\lambda)-\left(\frac{3 \cos i}{2 a^{2} \eta^{4}}\right) \lambda_{q_{1}} \\
& D_{65}^{(s p 1)}=\left(\frac{3 \cos i}{2 a^{2} \eta^{6}}\right)\left(4 q_{2} \epsilon_{3}-\eta^{2} \cos \theta\right)+\left(\frac{6 q_{2} \cos i}{a^{2} \eta^{6}}\right)(\theta-\lambda)-\left(\frac{3 \cos i}{2 a^{2} \eta^{4}}\right) \lambda_{q_{2}}
\end{aligned}
$$

where

$$
\begin{aligned}
\lambda & =\tan ^{-1}\left[\frac{r\left(1+\beta q_{1}^{2}\right) \sin \theta-\beta r q_{1} q_{2} \cos \theta+a q_{2}}{r\left(1+\beta q_{2}^{2}\right) \cos \theta-\beta r q_{1} q_{2} \sin \theta+a q_{1}}\right]-\frac{\eta \epsilon_{3}}{1+\epsilon_{2}} \\
\lambda_{q_{1}} & =\frac{q_{2}}{\eta(1+\eta)}+\frac{q_{1} V_{r}}{\eta V_{t}}-\frac{\eta r(a+r)}{a^{2} \eta^{4}}\left(q_{2}+\sin \theta\right) \\
\lambda_{q_{2}} & =-\frac{q_{1}}{\eta(1+\eta)}+\frac{q_{2} V_{r}}{\eta V_{t}}+\frac{\eta r(a+r)}{a^{2} \eta^{4}}\left(q_{1}+\cos \theta\right) \\
V_{r} & =\sqrt{\frac{\mu}{a \eta^{2}}} \epsilon_{3} \\
V_{t} & =\sqrt{\frac{\mu}{a \eta^{2}}}\left(1+\epsilon_{2}\right)
\end{aligned}
$$




$$
\begin{aligned}
r & =\frac{a \eta^{2}}{1+\epsilon_{2}} \\
\beta & =\frac{1}{\eta^{2}+\eta} \\
\epsilon_{1} & =\sqrt{q_{1}^{2}+q_{2}^{2}} \\
\epsilon_{2} & =q_{1} \cos \theta+q_{2} \sin \theta \\
\epsilon_{3} & =q_{1} \sin \theta-q_{2} \cos \theta \\
\eta & =\sqrt{1-q_{1}^{2}-q_{2}^{2}}
\end{aligned}
$$

Note that the expressions for the orbit elements variations $\boldsymbol{e}^{(s p 1)}=\left(a^{(s p 1)}, \theta^{(s p 1)}, i^{(s p 1)}\right.$, $\left.q_{1}^{(s p 1)}, q_{2}^{(s p 1)}, \Omega^{(s p 1)}\right)^{T}$ are given in appendix E.

\section{F.3 Short Period 2 Oscillations Matrix $D^{(s p 2)}$}

The non-zero elements of matrix $D^{(s p 2)}$ are

$$
\begin{aligned}
D_{11}^{(s p 2)}= & -\left(\frac{1}{a}\right) a^{(s p 2)} \\
D_{12}^{(s p 2)}= & \left(\frac{3 \sin ^{2} i}{2 a \eta^{6}}\right)\left(1+\epsilon_{2}\right)^{2}\left[2\left(1+\epsilon_{2}\right) \sin 2 \theta+3 \epsilon_{3} \cos 2 \theta\right] \\
D_{13}^{(s p 2)}= & -\left(\frac{3 \sin 2 i \cos 2 \theta}{2 a \eta^{6}}\right)\left(1+\epsilon_{2}\right)^{3} \\
D_{14}^{(s p 2)}= & -\left(\frac{9 \sin ^{2} i \cos 2 \theta}{2 a \eta^{8}}\right)\left(1+\epsilon_{2}\right)^{2}\left[2 q_{1}\left(1+\epsilon_{2}\right)+\eta^{2} \cos \theta\right] \\
D_{15}^{(s p 2)}= & -\left(\frac{9 \sin ^{2} i \cos 2 \theta}{2 a \eta^{8}}\right)\left(1+\epsilon_{2}\right)^{2}\left[2 q_{2}\left(1+\epsilon_{2}\right)+\eta^{2} \sin \theta\right] \\
D_{21}^{(s p 2)}= & -\left(\frac{2}{a}\right) \theta^{(s p 2)} \\
D_{22}^{(s p 2)}= & -\left(\frac{1}{8 a^{2} \eta^{4}}\right)\left\{3 ( 3 - 5 \operatorname { c o s } ^ { 2 } i ) \left[\left(q_{1} \cos \theta-q_{2} \sin \theta\right)+2 \cos 2 \theta+\right.\right. \\
& \left.\left(q_{1} \cos 3 \theta+q_{2} \sin 3 \theta\right)\right]-\sin ^{2} i\left[5\left(q_{1} \cos \theta-q_{2} \sin \theta\right)+16 \cos 2 \theta+\right. \\
& \left.\left.9\left(q_{1} \cos 3 \theta+q_{2} \sin 3 \theta\right)\right]\right\}
\end{aligned}
$$




$$
\begin{aligned}
D_{23}^{(s p 2)}= & -\left(\frac{\sin 2 i}{8 a^{2} \eta^{6}}\right)\left[10\left(q_{1} \sin \theta+q_{2} \cos \theta\right)+7 \sin 2 \theta+2\left(q_{1} \sin 3 \theta-q_{2} \cos 3 \theta\right)\right] \\
D_{24}^{(s p 2)}= & -\left[\frac{3-5 \cos ^{2} i}{8 a^{2} \eta^{6}}\right]\left\{4 q_{1}\left[3 \sin 2 \theta+q_{2}(3 \cos \theta-\cos 3 \theta)\right]+\left(\eta^{2}+4 q_{1}^{2}\right) \times\right. \\
& (3 \sin \theta+\sin 3 \theta)\}-\left[\frac{\sin ^{2} i}{8 a^{2} \eta^{2}(1+\eta)}\right](3 \sin \theta+\sin 3 \theta)-\left[\frac{\sin ^{2} i}{32 a^{2} \eta^{4}(1+\eta)}\right] \times \\
& \left\{36 q_{2}-4(2+3 \eta) \sin \theta-\left(39+12 \eta+\eta^{2}\right) \sin 3 \theta+9 \epsilon_{1} \sin 5 \theta+\right. \\
& 12 q_{2}\left(2 q_{1} \cos \theta+q_{2} \sin \theta\right)+9 q_{1}\left(q_{1} \sin 3 \theta-q_{2} \cos 3 \theta\right)+ \\
& 18\left(3 q_{1} \sin 4 \theta+2 q_{2} \cos 4 \theta\right)-3 q_{1}\left(q_{1} \sin 5 \theta-11 q_{2} \cos 5 \theta\right)+ \\
& \left.24\left[\left(1+\epsilon_{2}\right)\left(2+\epsilon_{2}\right) \sin \theta+\epsilon_{3}\left(3+2 \epsilon_{2}\right) \cos \theta\right] \cos 2 \theta\right\}-\left[\frac{3 \sin ^{2} i}{32 a^{2} \eta^{4}(1+\eta)^{2}}\right] \times \\
& {\left[4 \sin \theta-6 q_{1} \sin 4 \theta-q_{1}\left(q_{1} \sin 5 \theta+q_{2} \cos 5 \theta\right)\right]+\left[\frac{q_{1} \sin { }^{2} i}{8 a^{2} \eta^{6}(1+\eta)}\right] \times } \\
& {\left[20(1+\eta)\left(q_{1} \sin \theta+q_{2} \cos \theta\right)+32(1+\eta) \sin 2 \theta+3(4+3 \eta) \times\right.} \\
& \left.\left(q_{1} \sin 3 \theta-q_{2} \cos 3 \theta\right)\right]-\left[\frac{q_{1} \sin { }^{2} i(4+5 \eta)}{32 a^{2} \eta^{6}(1+\eta)^{2}}\right]\left\{24\left(q_{1} \sin \theta+q_{2} \cos \theta\right)+\right. \\
& 24 \epsilon_{3}\left(1+\epsilon_{2}\right)\left(2+\epsilon_{2}\right) \cos 2 \theta-(27+3 \eta)\left(q_{1} \sin 3 \theta-q_{2} \cos 3 \theta\right)-18 \sin 4 \theta- \\
& 3\left(q_{1} \sin 5 \theta+q_{2} \cos 5 \theta\right)+12 q_{2}\left[\left(3+\epsilon_{2}\right) q_{1}+3\left(q_{1} \cos 4 \theta+q_{2} \sin 4 \theta\right)+\right. \\
& \left.\left.q_{1}\left(q_{1} \cos 5 \theta+q_{2} \sin 5 \theta\right)\right]\right\} \\
&
\end{aligned}
$$




$$
\begin{aligned}
& D_{25}^{(s p 2)}=-\left[\frac{3-5 \cos ^{2} i}{8 a^{2} \eta^{6}}\right]\left\{4 q_{2}\left[3 \sin 2 \theta+q_{1}(3 \sin \theta+\sin 3 \theta)\right]+\left(\eta^{2}+4 q_{2}^{2}\right) \times\right. \\
& (3 \cos \theta-\cos 3 \theta)\}-\left[\frac{\sin ^{2} i}{8 a^{2} \eta^{2}(1+\eta)}\right](3 \cos \theta-\cos 3 \theta)-\left[\frac{\sin ^{2} i}{32 a^{2} \eta^{4}(1+\eta)}\right] \times \\
& \left\{36 q_{1}-4(2+3 \eta) \cos \theta+\left(39+12 \eta+\eta^{2}\right) \cos 3 \theta+9 \epsilon_{1} \cos 5 \theta+\right. \\
& 12 q_{1}\left(q_{1} \cos \theta+2 q_{2} \sin \theta\right)+9 q_{2}\left(q_{1} \sin 3 \theta-q_{2} \cos 3 \theta\right)+ \\
& 18\left(2 q_{1} \cos 4 \theta+7 q_{2} \sin 4 \theta\right)+3 q_{2}\left(11 q_{1} \sin 5 \theta-q_{2} \cos 5 \theta\right)+ \\
& \left.24\left[\epsilon_{3}\left(3+2 \epsilon_{2}\right) \sin \theta-\left(1+\epsilon_{2}\right)\left(2+\epsilon_{2}\right) \cos \theta\right] \cos 2 \theta\right\}-\left[\frac{3 \sin ^{2} i}{32 a^{2} \eta^{4}(1+\eta)^{2}}\right] \times \\
& {\left[4 \cos \theta-6 q_{2} \sin 4 \theta-q_{2}\left(q_{1} \sin 5 \theta+q_{2} \cos 5 \theta\right)\right]+\left[\frac{q_{2} \sin ^{2} i}{8 a^{2} \eta^{6}(1+\eta)}\right] \times} \\
& {\left[20(1+\eta)\left(q_{1} \sin \theta+q_{2} \cos \theta\right)+32(1+\eta) \sin 2 \theta+3(4+3 \eta) \times\right.} \\
& \left.\left(q_{1} \sin 3 \theta-q_{2} \cos 3 \theta\right)\right]-\left[\frac{q_{2} \sin ^{2} i(4+5 \eta)}{32 a^{2} \eta^{6}(1+\eta)^{2}}\right]\left\{24\left(q_{1} \sin \theta+q_{2} \cos \theta\right)+\right. \\
& 24 \epsilon_{3}\left(1+\epsilon_{2}\right)\left(2+\epsilon_{2}\right) \cos 2 \theta-(27+3 \eta)\left(q_{1} \sin 3 \theta-q_{2} \cos 3 \theta\right)-18 \sin 4 \theta- \\
& 3\left(q_{1} \sin 5 \theta+q_{2} \cos 5 \theta\right)+12 q_{2}\left[\left(3+\epsilon_{2}\right) q_{1}+3\left(q_{1} \cos 4 \theta+q_{2} \sin 4 \theta\right)+\right. \\
& \left.\left.q_{1}\left(q_{1} \cos 5 \theta+q_{2} \sin 5 \theta\right)\right]\right\} \\
& D_{31}^{(s p 2)}=-\left(\frac{2}{a}\right) i^{(s p 2)} \\
& D_{32}^{(s p 2)}=\left(\frac{3 \sin 2 i}{8 a^{2} \eta^{4}}\right)\left[\left(q_{1} \sin \theta+q_{2} \cos \theta\right)+2 \sin 2 \theta+\left(q_{1} \sin 3 \theta-q_{2} \cos 3 \theta\right)\right] \\
& D_{33}^{(s p 2)}=-\left(\frac{\cos 2 i}{4 a^{2} \eta^{4}}\right)\left[3\left(q_{1} \cos \theta-q_{2} \sin \theta\right)+3 \cos 2 \theta+\left(q_{1} \cos 3 \theta+q_{2} \sin 3 \theta\right)\right] \\
& D_{34}^{(s p 2)}=-\left(\frac{\sin 2 i}{8 a^{2} \eta^{6}}\right)\left\{4 q_{1}\left[3 \cos 2 \theta-q_{2}(3 \sin \theta-\sin 3 \theta)\right]+\left(\eta^{2}+4 q_{1}^{2}\right) \times\right. \\
& (3 \cos \theta+\cos 3 \theta)\} \\
& D_{35}^{(s p 2)}=-\left(\frac{\sin 2 i}{8 a^{2} \eta^{6}}\right)\left\{4 q_{2}\left[3 \cos 2 \theta+q_{1}(3 \cos \theta+\cos 3 \theta)\right]-\left(\eta^{2}+4 q_{2}^{2}\right) \times\right. \\
& (3 \sin \theta-\sin 3 \theta)\} \\
& D_{41}^{(s p 2)}=-\left(\frac{2}{a}\right) q_{1}^{(s p 2)}
\end{aligned}
$$




$$
\begin{aligned}
& D_{42}^{(s p 2)}=\left[\frac{3 q_{2}\left(3-5 \cos ^{2} i\right)}{8 a^{2} \eta^{4}}\right]\left[\left(q_{1} \cos \theta-q_{2} \sin \theta\right)+2 \cos 2 \theta+\left(q_{1} \cos 3 \theta+q_{2} \sin 3 \theta\right)\right]+ \\
& \left(\frac{3 \sin ^{2} i}{32 a^{2} \eta^{4}}\right)\left\{2 \left[2 q_{2} \epsilon_{2}-9 q_{2}\left(q_{1} \cos 3 \theta+q_{2} \sin 3 \theta\right)+12\left(q_{1} \sin 4 \theta-q_{2} \cos 4 \theta\right)-\right.\right. \\
& \left.5 q_{2}\left(q_{1} \cos 5 \theta+q_{2} \sin 5 \theta\right)\right]+\left[4\left(1+3 q_{1}^{2}\right) \sin \theta+40 q_{1} \sin 2 \theta+\right. \\
& \left.\left.\left(28+17 \epsilon_{1}\right) \sin 3 \theta+5 \epsilon_{1} \sin 5 \theta\right]\right\} \\
& D_{43}^{(s p 2)}=-\left(\frac{\sin 2 i}{32 a^{2} \eta^{4}}\right)\left\{2 \left[36 q_{1}\left(q_{1} \cos \theta-q_{2} \sin \theta\right)+30\left(q_{1} \cos 2 \theta-q_{2} \sin 2 \theta\right)-\right.\right. \\
& \left.q_{2}\left(q_{1} \sin 3 \theta-q_{2} \cos 3 \theta\right)+9\left(q_{1} \cos 4 \theta+q_{2} \sin 4 \theta\right)+3 q_{2}\left(q_{1} \sin 5 \theta-q_{2} \cos 5 \theta\right)\right]+ \\
& {\left[6 q_{1}\left(3+2 q_{1} \cos \theta\right)+12\left(1-4 \epsilon_{1}\right) \cos \theta+\left(28+17 \epsilon_{1}\right) \cos 3 \theta+\right.} \\
& \left.\left.3 \epsilon_{1} \cos 5 \theta\right]\right\} \\
& D_{44}^{(s p 2)}=\left[\frac{q_{2}\left(3-5 \cos ^{2} i\right)}{8 a^{2} \eta^{6}}\right]\left\{4 q_{1}\left[3 \sin 2 \theta+q_{2}(3 \cos \theta-\cos 3 \theta)\right]+\left(\eta^{2}+4 q_{1}^{2}\right) \times\right. \\
& (3 \sin \theta+\sin 3 \theta)\}-\left(\frac{\sin ^{2} i}{8 a^{2} \eta^{4}}\right)\left\{\left[8 q_{1} \cos 3 \theta-3 q_{2}(\sin \theta-\sin 3 \theta)\right]+\right. \\
& \left.3\left[5+\epsilon_{2}+3 \cos 2 \theta+3\left(q_{1} \cos 3 \theta+q_{2} \sin 3 \theta\right)\right] \cos 2 \theta\right\}-\left(\frac{3 q_{1} \sin ^{2} i}{4 a^{2} \eta^{6}}\right) \times \\
& \left\{2 q_{1}\left[\left(q_{1} \cos \theta-q_{2} \sin \theta\right)+\left(q_{1} \cos 3 \theta+q_{2} \sin 3 \theta\right)\right]+[9 \cos \theta-\cos 3 \theta+\right. \\
& \left.2 q_{1}\left(5+\epsilon_{2}\right)+6\left(q_{1} \cos 2 \theta+q_{2} \sin 2 \theta\right)+2 q_{1}\left(q_{1} \cos 3 \theta+q_{2} \sin 3 \theta\right)\right] \times \\
& \cos 2 \theta\} \\
& D_{45}^{(s p 2)}=\left[\frac{3-5 \cos ^{2} i}{8 a^{2} \eta^{6}}\right]\left\{\left(\eta^{2}+4 q_{2}^{2}\right)\left[3 \sin 2 \theta+q_{1}(3 \sin \theta+\sin 3 \theta)\right]+\right. \\
& \left.2 q_{2}\left(\eta^{2}+2 q_{2}^{2}\right)(3 \cos \theta-\cos 3 \theta)\right\}+\left(\frac{\sin ^{2} i}{16 a^{2} \eta^{4}}\right)\left\{\left[6\left(q_{1} \sin \theta+2 q_{2} \cos \theta\right)-\right.\right. \\
& \left.\left(9 q_{1} \sin 3 \theta+q_{2} \cos 3 \theta\right)-9 \sin 4 \theta-3\left(q_{1} \sin 5 \theta+q_{2} \cos 5 \theta\right)\right]-\left(\frac{3 q_{2} \sin ^{2} i}{8 a^{2} \eta^{6}}\right) \times \\
& \left\{2 q _ { 1 } \left[3+2\left(2 q_{1} \cos \theta-q_{2} \sin \theta\right)+10 \cos 2 \theta+3\left(q_{1} \cos 3 \theta+q_{2} \sin 3 \theta\right)+\right.\right. \\
& \left.\left(q_{1} \cos 5 \theta+q_{2} \sin 5 \theta\right)\right]+\left[8 \cos \theta+9 \cos 3 \theta+6\left(q_{1} \cos 4 \theta+q_{2} \sin 4 \theta\right)-\right. \\
& \cos 5 \theta]\}
\end{aligned}
$$




$$
\begin{aligned}
& D_{51}^{(s p 2)}=-\left(\frac{2}{a}\right) q_{2}^{(s p 2)} \\
& D_{52}^{(s p 2)}=-\left[\frac{3 q_{1}\left(3-5 \cos ^{2} i\right)}{8 a^{2} \eta^{4}}\right]\left[\left(q_{1} \cos \theta-q_{2} \sin \theta\right)+2 \cos 2 \theta+\left(q_{1} \cos 3 \theta+q_{2} \sin 3 \theta\right)\right]+ \\
& \left(\frac{3 \sin ^{2} i}{32 a^{2} \eta^{4}}\right)\left\{2 \left[2 q_{1} \epsilon_{2}+9 q_{1}\left(q_{1} \cos 3 \theta+q_{2} \sin 3 \theta\right)-12\left(q_{1} \cos 4 \theta+q_{2} \sin 4 \theta\right)-\right.\right. \\
& \left.5 q_{1}\left(q_{1} \cos 5 \theta+q_{2} \sin 5 \theta\right)\right]+\left[4\left(1+3 q_{2}^{2}\right) \cos \theta+40 q_{2} \sin 2 \theta-\right. \\
& \left.\left.\left(28+17 \epsilon_{1}\right) \cos 3 \theta+5 \epsilon_{1} \cos 5 \theta\right]\right\} \\
& D_{53}^{(s p 2)}=-\left(\frac{\sin 2 i}{32 a^{2} \eta^{4}}\right)\left\{2 \left[36 q_{1}\left(q_{1} \sin \theta+q_{2} \cos \theta\right)+30\left(q_{1} \sin 2 \theta+q_{2} \cos 2 \theta\right)+\right.\right. \\
& \left.q_{1}\left(q_{1} \sin 3 \theta-q_{2} \cos 3 \theta\right)+9\left(q_{1} \sin 4 \theta-q_{2} \cos 4 \theta\right)+3 q_{1}\left(q_{1} \sin 5 \theta-q_{2} \cos 5 \theta\right)\right]- \\
& {\left[6 q_{2}\left(3+2 q_{2} \sin \theta\right)+12\left(1+2 \epsilon_{1}\right) \sin \theta-\left(28+17 \epsilon_{1}\right) \sin 3 \theta+\right.} \\
& \left.\left.3 \epsilon_{1} \sin 5 \theta\right]\right\} \\
& D_{54}^{(s p 2)}=-\left[\frac{3-5 \cos ^{2} i}{8 a^{2} \eta^{6}}\right]\left\{\left(\eta^{2}+4 q_{1}^{2}\right)\left[3 \sin 2 \theta+q_{2}(3 \cos \theta-\cos 3 \theta)\right]+\right. \\
& \left.2 q_{1}\left(\eta^{2}+2 q_{1}^{2}\right)(3 \sin \theta+\sin 3 \theta)\right\}-\left(\frac{\sin ^{2} i}{16 a^{2} \eta^{4}}\right)\left\{\left[6\left(2 q_{1} \sin \theta+q_{2} \cos \theta\right)+\right.\right. \\
& \left.\left(q_{1} \sin 3 \theta+9 q_{2} \cos 3 \theta\right)+9 \sin 4 \theta-3\left(q_{1} \sin 5 \theta+q_{2} \cos 5 \theta\right)\right]+\left(\frac{3 q_{1} \sin ^{2} i}{8 a^{2} \eta^{6}}\right) \times \\
& \left\{2 q _ { 2 } \left[3-2\left(q_{1} \cos \theta-2 q_{2} \sin \theta\right)-10 \cos 2 \theta-3\left(q_{1} \cos 3 \theta+q_{2} \sin 3 \theta\right)+\right.\right. \\
& \left.\left(q_{1} \cos 5 \theta+q_{2} \sin 5 \theta\right)\right]+\left[8 \sin \theta-9 \sin 3 \theta-6\left(q_{1} \sin 4 \theta-q_{2} \cos 4 \theta\right)-\right. \\
& \sin 5 \theta]\} \\
& D_{55}^{(s p 2)}=-\left[\frac{q_{1}\left(3-5 \cos ^{2} i\right)}{8 a^{2} \eta^{6}}\right]\left\{4 q_{2}\left[3 \sin 2 \theta+q_{1}(3 \sin \theta+\sin 3 \theta)\right]+\left(\eta^{2}+4 q_{2}^{2}\right) \times\right. \\
& (3 \cos \theta-\cos 3 \theta)\}-\left(\frac{\sin ^{2} i}{8 a^{2} \eta^{4}}\right)\left\{\left[8 q_{2} \sin 3 \theta+3 q_{1}(\cos \theta+\cos 3 \theta)\right]+\right. \\
& \left.3\left[5+\epsilon_{2}-3 \cos 2 \theta-\left(q_{1} \cos 3 \theta-q_{2} \sin 3 \theta\right)\right] \cos 2 \theta\right\}-\left(\frac{3 q_{2} \sin ^{2} i}{4 a^{2} \eta^{6}}\right) \times \\
& {\left[9 \sin \theta-\sin 3 \theta+2 q_{2}\left(5+\epsilon_{2}\right)+6\left(q_{1} \sin 2 \theta-q_{2} \cos 2 \theta\right)+\right.} \\
& \left.2 q_{1}\left(q_{1} \sin 3 \theta-q_{2} \cos 3 \theta\right)\right] \cos 2 \theta
\end{aligned}
$$




$$
\begin{aligned}
D_{61}^{(s p 2)}= & -\left(\frac{2}{a}\right) \Omega^{(s p 2)} \\
D_{62}^{(s p 2)}= & -\left(\frac{3 \cos i}{4 a^{2} \eta^{4}}\right)\left[\left(q_{1} \cos \theta-q_{2} \sin \theta\right)+2 \cos 2 \theta+\left(q_{1} \cos 3 \theta+q_{2} \sin 3 \theta\right)\right] \\
D_{63}^{(s p 2)}= & \left(\frac{\sin i}{4 a^{2} \eta^{4}}\right)\left[3\left(q_{1} \sin \theta+q_{2} \cos \theta\right)+3 \sin 2 \theta+\left(q_{1} \sin 3 \theta-q_{2} \cos 3 \theta\right)\right] \quad \text { (H.18b) } \\
D_{64}^{(s p 2)}= & -\left(\frac{\cos i}{4 a^{2} \eta^{6}}\right)\left\{4 q_{1}\left[3 \sin 2 \theta+q_{2}(3 \cos \theta-\cos 3 \theta)\right]+\left(\eta^{2}+4 q_{1}^{2}\right) \times\right. \\
& (3 \sin \theta+\sin 3 \theta)\} \\
D_{65}^{(s p 2)}= & -\left(\frac{\cos i}{4 a^{2} \eta^{6}}\right)\left\{4 q_{2}\left[3 \sin 2 \theta+q_{1}(3 \sin \theta+\sin 3 \theta)\right]+\left(\eta^{2}+4 q_{2}^{2}\right) \times\right. \\
& (3 \cos \theta-\cos 3 \theta)\}
\end{aligned}
$$

where

$$
\begin{aligned}
\epsilon_{1} & =\sqrt{q_{1}^{2}+q_{2}^{2}} \\
\epsilon_{2} & =q_{1} \cos \theta+q_{2} \sin \theta \\
\epsilon_{3} & =q_{1} \sin \theta-q_{2} \cos \theta \\
\eta & =\sqrt{1-q_{1}^{2}-q_{2}^{2}}
\end{aligned}
$$

Note that the expressions for the orbit elements variations $e^{(s p 2)}=\left(a^{(s p 2)}, \theta^{(s p 2)}, i^{(s p 2)}\right.$, $\left.q_{1}^{(s p 2)}, q_{2}^{(s p 2)}, \Omega^{(s p 2)}\right)^{T}$ are given in appendix E. 